


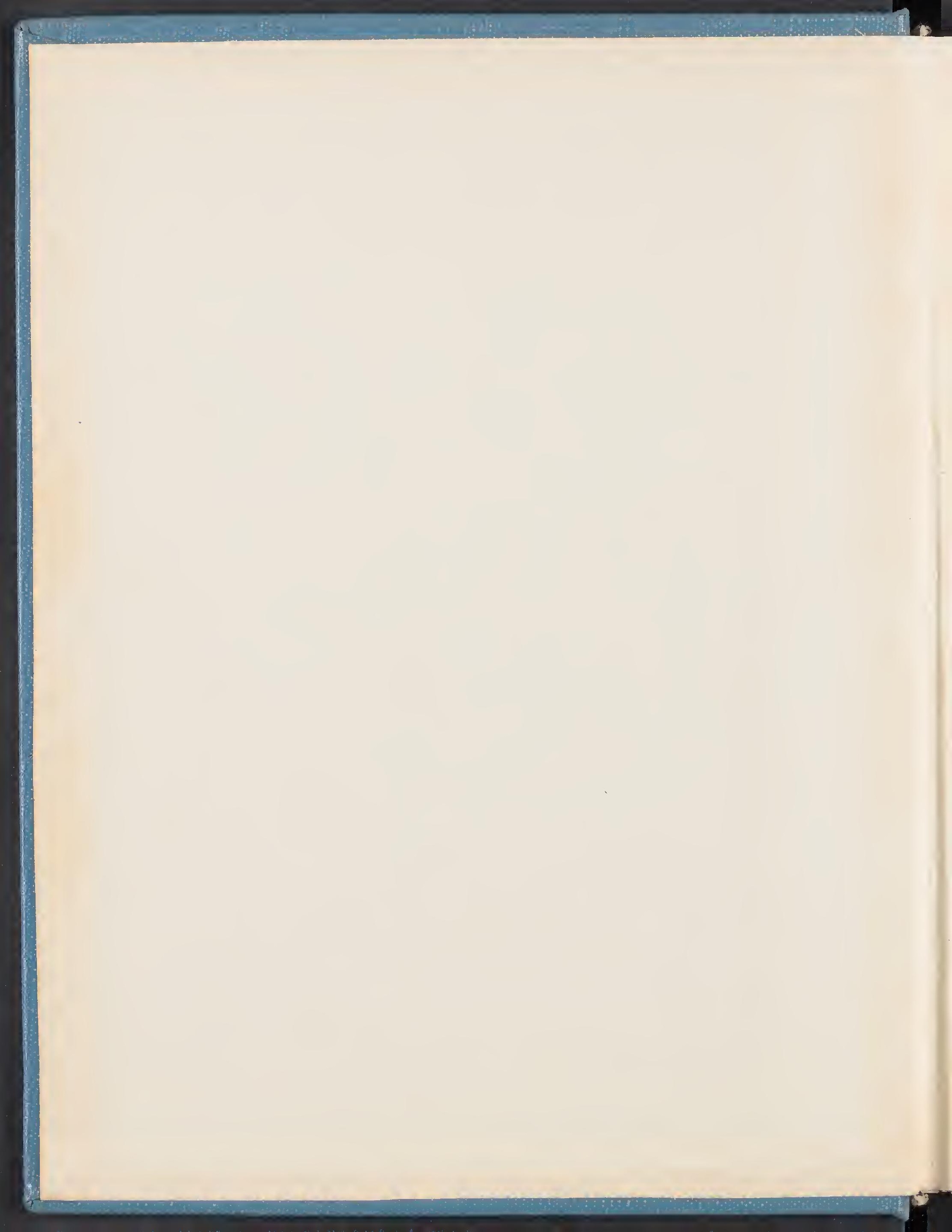






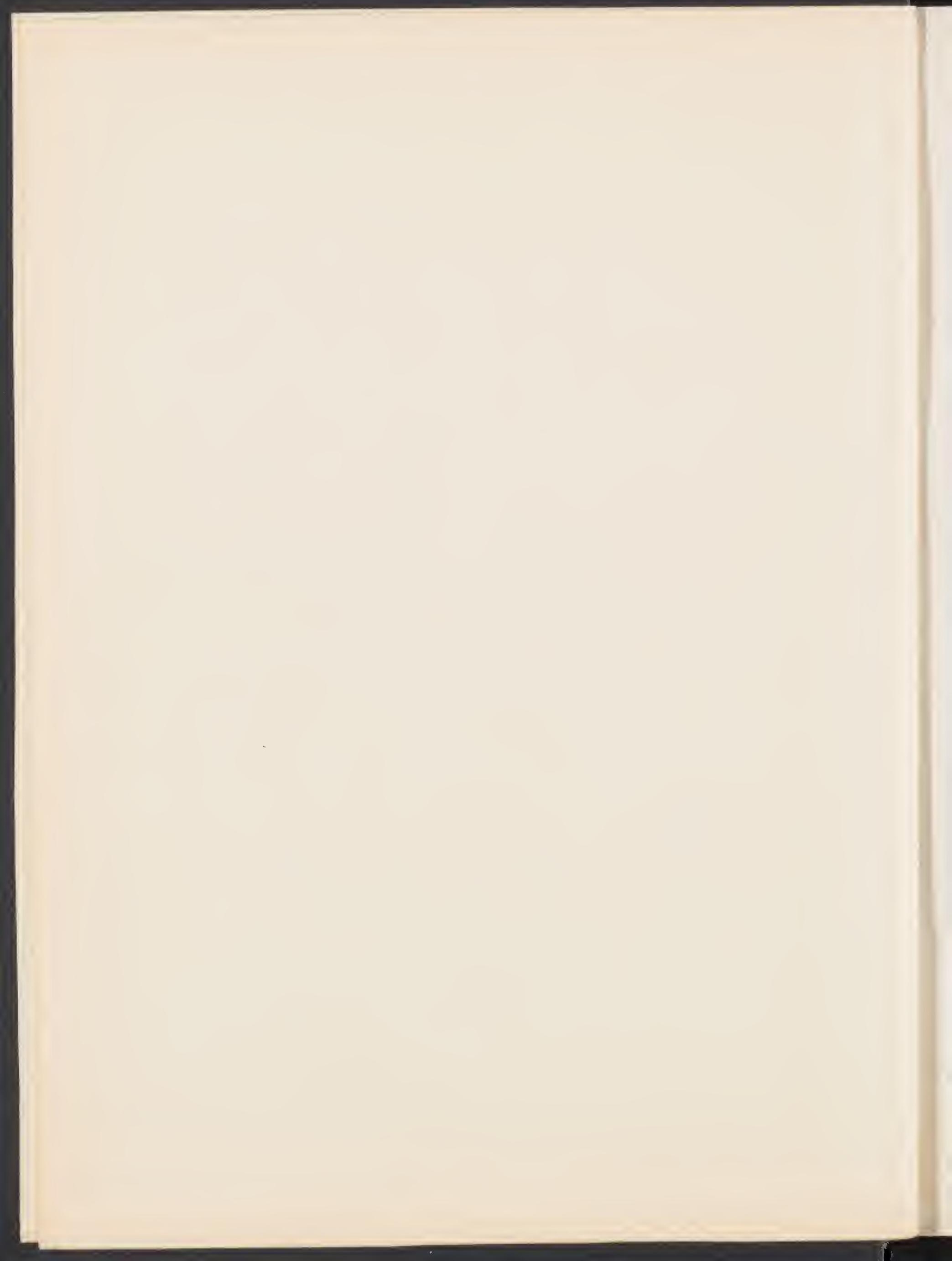


COSTA RICAIT

AGARICACEAE

by

CARROII W. DODGE

$1929-1936$ 
QK

629

A4

D 6443

1940x

D 6443

V. 1

RARE EOT 


\section{COSTA RICAN AGARICACEAE}

Carroll w. Dodge

The first collection of Costa Rican Agaricaceae of which records have survied, was that of Anders S. Oersted who visited Costa Rica, Nicaragua and the Virgin Islands in 1846-1348. *

Dersted, Anders S. 1351. In reise i Guanacaste. Tidskr. f. populaer Naturvidenskab $1: 21-35$. 1 map. 1851 .

-.-.. 1851. Naturens Physiognomie i Central America. Ibid. 1:255283.

Upon his return to Denmark, he anparently turned over his collections and a duplicate set of water-color paintings to Elias Fries for naming, since the form the greater part of the Agaricaceae treated in his "Novae Symbolae lincologicae published in 1851. No specimens have survived at Uppsala, although fries mentions alcoholic material in alcohol. The collection of drawings, mostly on sheets $x \mathrm{~cm}$, usually with Agaricus and a number written across the top in what may be Oersted's handwriting. The number corresponds to that cited by Fries. Below the drawing, the name given by Fries appears, usually in a handwriting anparently that of Fries in his later years. At the bottom of the sheets are the data: Costa Rica. A. . Oersted in the handwriting of $T$. M. Fries. This latter was probably added when the collection was tistributed in the general Friesian collection of drawings which were arranged by genera in portfolios in the early $1880^{\prime} \mathrm{s}$. Since many of the species cited are not from Costa Rica, the data given in the Novae Symbolae shoudd be considered correct and not the locality given on the sheet. All of the species but one in which Fries mentions a drawing are present, along wiht some drawings of some fungi which are not determinable in the absence of specimens. 


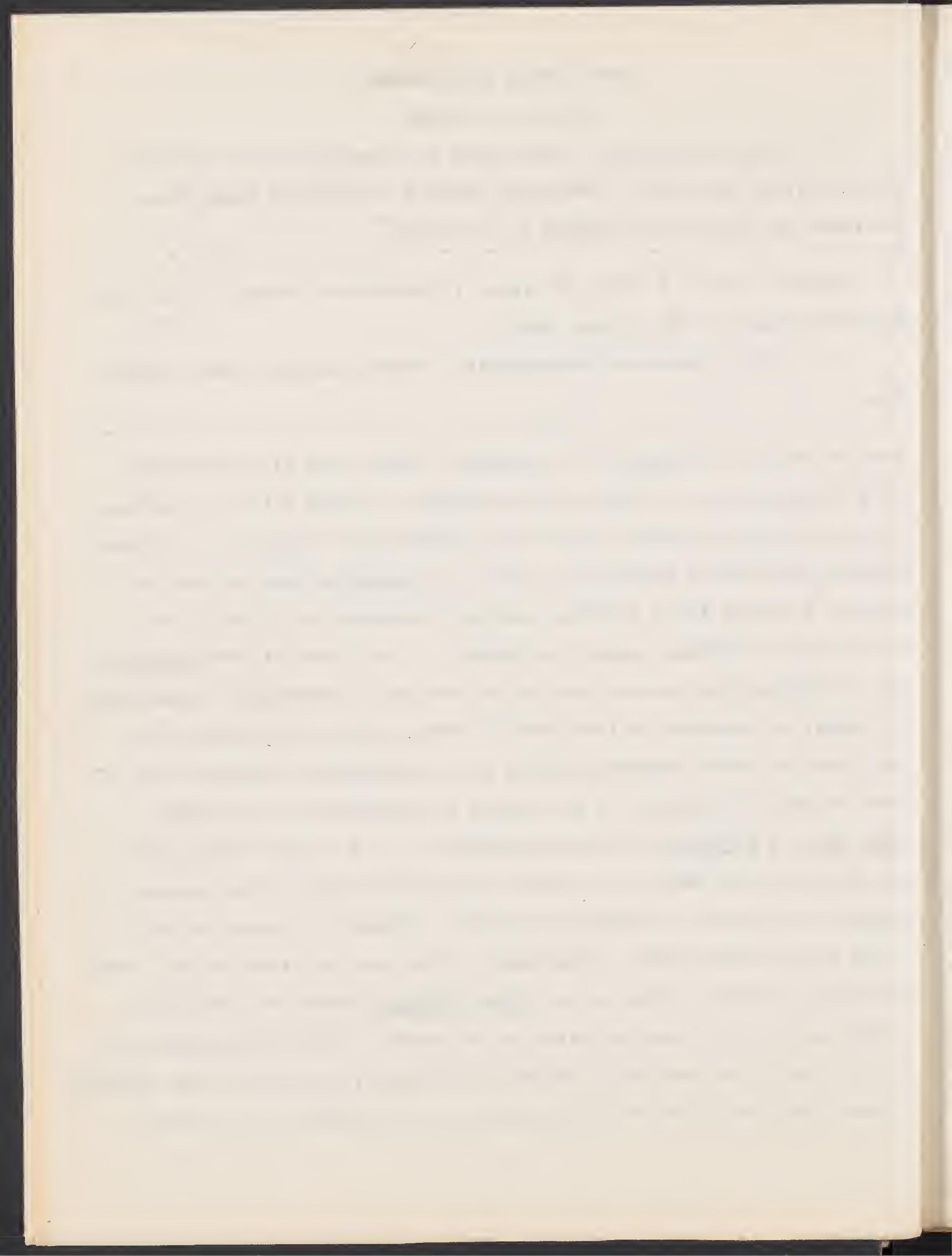


Hymenium plicate, folds obtuse

Spores green, stipe central

Spores hyaline or ochraceous

Hymenophore leather $y$, reviving

stipe lateral or wanting

Lamellae white, spores ovoid

Lamellae fuscous, spores ovoid

Lamellae brick-red, spores spher ical

Stipe central, lamellae brownish

Hymenophore woody, applanate

Hymenophore fleshy

Pileus thin, membranous, infundibuliform

Pileus thick, fleshy, terricale

Pileus convex, orange buff, gills orange

Pileus plane

Gills warm buff, pileus avellaneous

Gills luteous or paler, pileus

Pileus infundibuliform

Gills orange buff

Gills grayish fuscous, turbinate

Gills clay color, stipe cartilaginous tough, per haps is Lentinus

Hymenium with well developed gills

Spores white

Hymenophore tough to woody

Lamellae split longitudinally

Margin entire or somewhat lobes, deeply

tanentose

Margin split halfway to attachment,

lobes soon nude

Lamellae not slplitting longitudinally

Stipe lateral or wanting, pileus irregular

Pileus sessile, resupinate when young

Context homogeneous

Pileus membranous

Pileus pale brownish, 5-10mm., decorated with long fibers

$P$ ileus white or whitish

Pileus 2-6m, surface pruinose floccose

Pileus not more than $2 m m$

Surface floccose

Surface pruinose to glabrous

Hymenophores on arachnoid my-

$$
\text { celium }
$$

Not as above

Pileus coriaceous

Pileus \& gills white or yellowish

Pileus \& gills isabelline

Pileus \& gills blood-red

Context with upper layer gelified

Pil eus $1.5-5 \mathrm{~cm}$, gills white

Surface white, floccose fibrillose

Surface warm buff to light buff, very gelified, gills veryynarrow and close

Pileus grayish, smooky, pruinose, gills distant
Chlorophyllum viride (Pat)Murr.

Xerotus Fr. split as follows Plicatura.

P. guadelupensis (Pat.)Murr.

P. Mauryi (Pat.)

P. latericia (B.\&C.) Murr. Xerotinus martinicensis (Pat.) Neurophyllum ochraceum Pat.

Trogia ciner ea Pat.

Canthar ellus

C. Sept. 11

c. July 7

C. cibarius

C. 5132

C. mexicanus

C. Aug. 27
Schizophyllum

S. commune

S. radiaturn Fr.
Pl eurotopsis

P. asperifolia

P.liliputiana $7301,7304,7260$

P. niduliformis

P. arachnoidea

P. calospora

Scytinotus

S. distantifolius

S. concolor

S. haematodes

Pleurotus sec. Resupinatus

P. cubensis

P. July 14

P. subbarbatus 


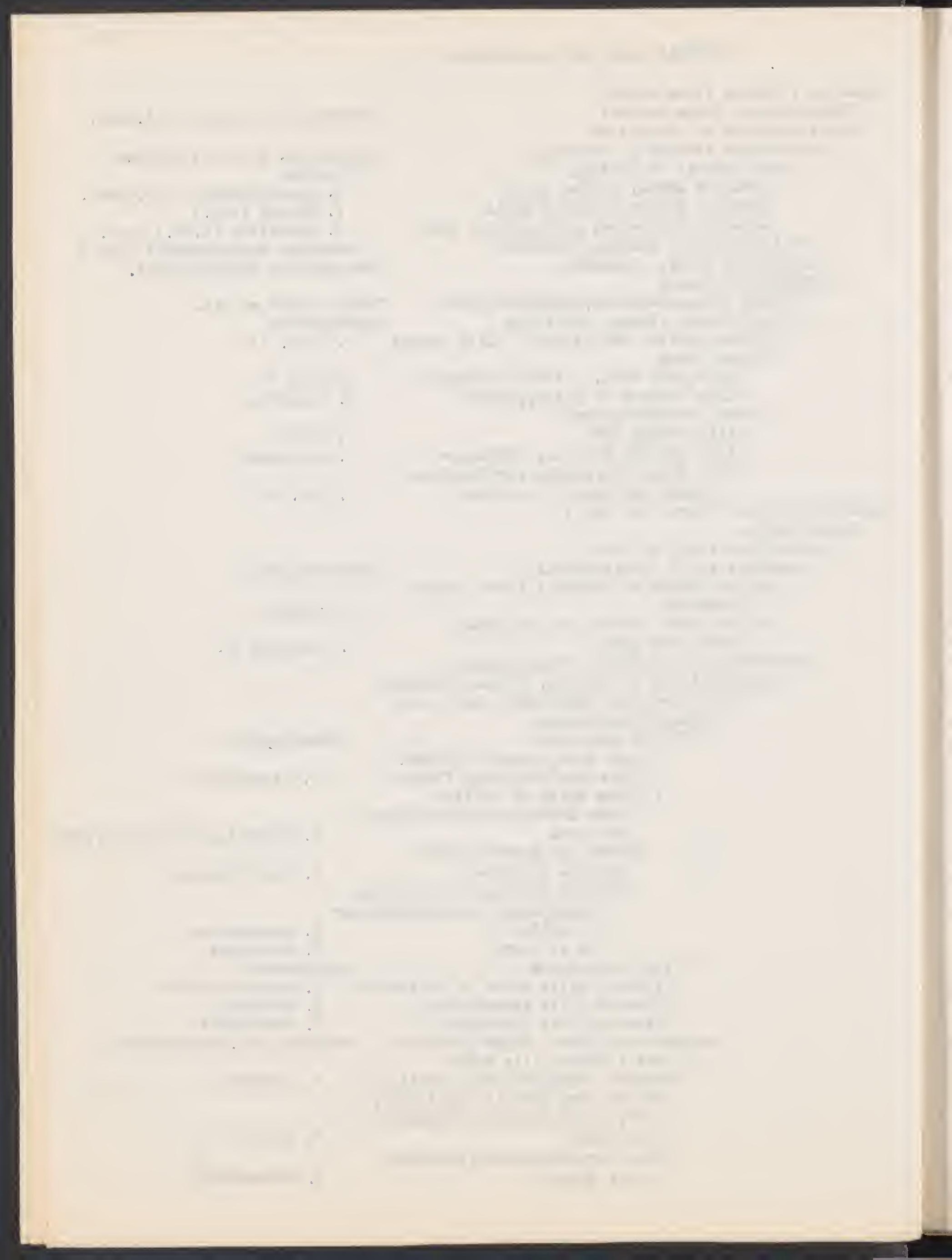


Pileus $4-8 \mathrm{~mm}$

Gills white, pileus black, campanulate P.orizabensis

Gills and pileus blackish brom, flabelliform

P. subbarbatus

Pileus stipitate or dimidiate, not resupinate when young

Pileus membranous, stipe tubular

Pileus 2-3mm

Pileus 10 mor more in diameter

Pileus light brow, glabrous

Pileus purple, tomentose

Marasmiellus

Pileus pale to light ochraceous salmon, velvety $M .4658$

Pileus fleshy tought to coriaceous, stipe solid when present

Gills rather distant and broad

Panellus

Gills ver y crowded and narrow

Pileus avellaneous

Pileus white or yellowish

Gills dichotonous, edges obtuse

Gills not dichotomous, edges thin

stipe tomentose, distinct

Stipe glabrous, usually only a tubercle

P. eugrammus

P. jalapensis

P. cantharelloides

P. subcantharelloides

P. flabellatus

Stipe central to slightly excentric, pileus regular

stipe tubular, slender with cartilaginous cortex

Pileus somewhat gelified, stipe horny

Gills few, distant

Terrestrial; gills decurrent

Growing on wood

Pileus white or pale,not darkening ondrying

Pileus less than $1 \mathrm{~cm}$; gills decurrent

Pileus $1-2 \mathrm{~cm}$.; gills adnate

Pileus some shade of avellaneous or pale bay or becoming so on drying
Pileus $2 \mathrm{~cm}$. or less

Pileus $3-6 \mathrm{~cm}$.

Pileus deeply umbilicate to infundibulifor

Pileus plane or slightly depressed

Pileus plae or tinged yellow when dry

Pileus latericious when dry

Heliomyces

H. terrestris

H. translucens

H. striatus

H. subavel laneous

H. subspodoides 726

H. hondur ensis

H. crenulatus

H. Peckii

H. rubritinctus

Gills numerous, crowded, growing on wood

Hymenophore solitary

Hymenophores cespitose

Pileus umbilicate, gills interveined

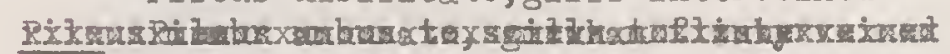

Pileus umbonate, gills not interveined

Pileus menbranous to somerhat eleshy

Pileus glabrous or nearly so

Stipe compound or branched

Pileus 6rm

Pileus $2 \mathrm{~mm}$ in diameter

Surface white, bay on umbo

surface bay throughout

stipe simple, unbranched

H. angustifolius

H. multifolius

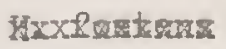

F. foetens 


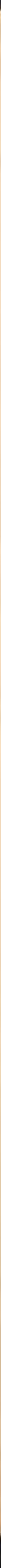


Pileus membranous to somewhat fleshy, stipe simple-Marasmius

Pilens minute, $1-3 \mathrm{~mm}$. broad (reaching $4 \mathrm{~mm}$. in M. Hioramj)

Surface of pileus white or whjtish rarely darker on the disk. Disk blackish and papiliate

Disk white or paliid.

Stipe 4-10 mm. Ione.

Pileus $1-2 \mathrm{~mm}$. broad.

Surface subpellucid, pruinose.

Surface opaue, flabrous.

pileus 2-3mm.broad.

Surface subtranslucent, elabrous.

Surface opaue, fulverulent.

Stipe 10-20 mm, 10ne.

M. Peckii.

Pileus umbilicate

Stipe pallid, $1-1.5 \mathrm{~cm} .1$ one.

M. petiolorum.

Mi. Marbleae.

Stipe fulvous, $3.5 \mathrm{~cm}$. or more long.

Pileus not umbilicate.

Stipe rallid throughout.

stipe pale-yellow below.

Surface of pileus pale-yellow.

Lamellae adnate; pileus $3 \mathrm{~mm}$. broad.

Lamellae decurrent; pileus $1 \mathrm{~mm}$. broad

Surface of pileus brownish-cinnamon;pileus

ii. subtenerrimus.

Mi. bermudensis.

convex;lamellee djstant, pallid;stipe $2 \mathrm{~mm}$. lone.

Surface of wileus umbrinous; pileus hemisphericjlamellae few, palid.

Surface of pileus bay, blackish and papillate

at the center, pileus $2-4 \mathrm{~mm}$. hroad.

Pileus small, 3-10 mm,broad

Surface of pileus white or pallid, rarely

slithtly darker at the center.

stipe white or pallid throuchout.

stipe gulabrous.

Lamellae free; stipe 10-12 mm.Ionc.

Lamellae decurrent; stipe $1.5-4 \mathrm{~cm}$. Ione.

Ttistipet 1,5e2. cinonlane.

Stipe $4 \mathrm{~cm}$. Ione.

Stile minutely pruinose or floccose,

sometimes becoming subejabrous.

Pileus scarcely $5 \mathrm{~mm}$. hroad, not

striate;lamellae crowded.

Pileus 6-10 mm. broad, striate; lamellae distant.

stipe brownish or blackish below.

Iin. theobromicola.

M. chrysochaetes.

M. proletarius.

$N_{*}$, ineevuilis.

M. subelobosus.

in. flavellus.

x. .

M. nanus.

IV. guyanensis. 7244

M. Hiorami.

M.stylobates.

M. praedecurrens.

M. niveicolor.

Stipe $1 \mathrm{~cm}$, or less long.

Lamellae adnate;pileus usually darker on the disk.

Lamellae decurrent;pileus not darker on the disk.

Stipe $2-3 \mathrm{~cm}$. Iong. ilamellae adnate.

Pileus umbilicate, unicolorous.

Pileus umbonate, slithtly darker at the center.

d.. Sotoges

II. setulosipes.

M. synodicus. 
Stipe 5-8 cm. Iong: lamellae free;plant terrestrial

Surface of pileus pale-avellaneous or pale

isabelline, fading to white on dryine.

Stipe $1 \mathrm{~cm}$. Ione.

Stipe $4 \mathrm{~cm}$. IonE.

surface of pileus ochraceous, darker on the

disk; pileus 2-4 mm.broad, stipe blackish,

$1 \mathrm{~cm}$. Ione.

Surface of pileus fulvous (tamm)

Pileus $3-4 \mathrm{~mm}$. broad; densely cespitose.

Pileus 6-10 mn. broad.

Stipe pelizucid.

Stipe fulvous to brown.

Pileus $6 \mathrm{~mm}$. broad; lameliae crowded.

Pileus $10 \mathrm{~mm}$. broad; lamellae distont.

Stive blackish

Stipe reddish-brow.

Surface of nileus avellaneous to umbrinous

or brow, often tinged with rose or bay, not

fadine to white on drying.

Stipe less than $2 \mathrm{~cm}$. lone.

Stipe black.

Sti.je not black.

Iramellae cream-colored.

Lamellae pale-fuscous.

stipe more than $2 \mathrm{~cm}$. lone.

stipe $2-3 \mathrm{~cm}$. Ione.

Stipe $4-6 \mathrm{~cm}$. lone.

surface of pileus h]ackish-blue-ereen; wileus

$8 \mathrm{~mm}$. broad; stip $1 \% \mathrm{~mm}$. lone.

M. Elebigenus.

1. raminis.

M. subrotula.7310

M. Crescentiae.

M. aciculaeformis

M. hinnuleus.

M. personatus.

N. sulcatipes.

vi. rugulosus.

H. musicola.

Surface of pileus nale-reci, fading to isabelline

on drying; Nileus $5-8 \mathrm{~mm}$. broad; stipe $2-2.5$

cm. Ione

Surface of pileus bay.

Pileus $5 \mathrm{~mm}$. broad.

Pileus 5-10 min. broad.

Iamellae jaliid; stipe clabrous.

Iame l. de adnexed; stipe $0.5-1 \mathrm{~mm}$. thick
Lamellade adnate to a slicht collar;stive $0.5 \mathrm{~m}$. thick.

Lamel.iae fulvous; stipe pubescent.

I9. picipes.

Ii. Underwoodii.

in. tenebrarum, 7245

Ni. colimensis.

M. soliformis

L. atroviridis.

M. pallescens.

M. jamaicensisis

V. Vilsonij.

Ni. paucifolia.

Vi. portoricensis. Surface of pileus dark-purple;lamel.lae few,

1 dark purple on ederes when young.

Pileus medium to laree, more than $1 \mathrm{~cm}$. broad.

Surface of pileus mostly white or pallid, sometimes darker at the center.

pileus becomine eccentric;stife usually rather

short and curved.

stipe white above, reddish-brow below.

M. stenophyllus.

stine rosy-isabejline throughout,

Pileus $1-1.5 \mathrm{~cm}$. broad.

Pileus $2 \mathrm{~cm}$. broad.

M. troyanus.

M. subplexifolius

Pileus never eccentric; stine long, erect.

Pileus plane, umbonate, $12-18 \mathrm{~mm}$, broad;

lamellae distant.

Stipe $0.5 \mathrm{~mm}$, thick

Stipe $1-1.5 \mathrm{~mm}$. thick

vivofusgus. 


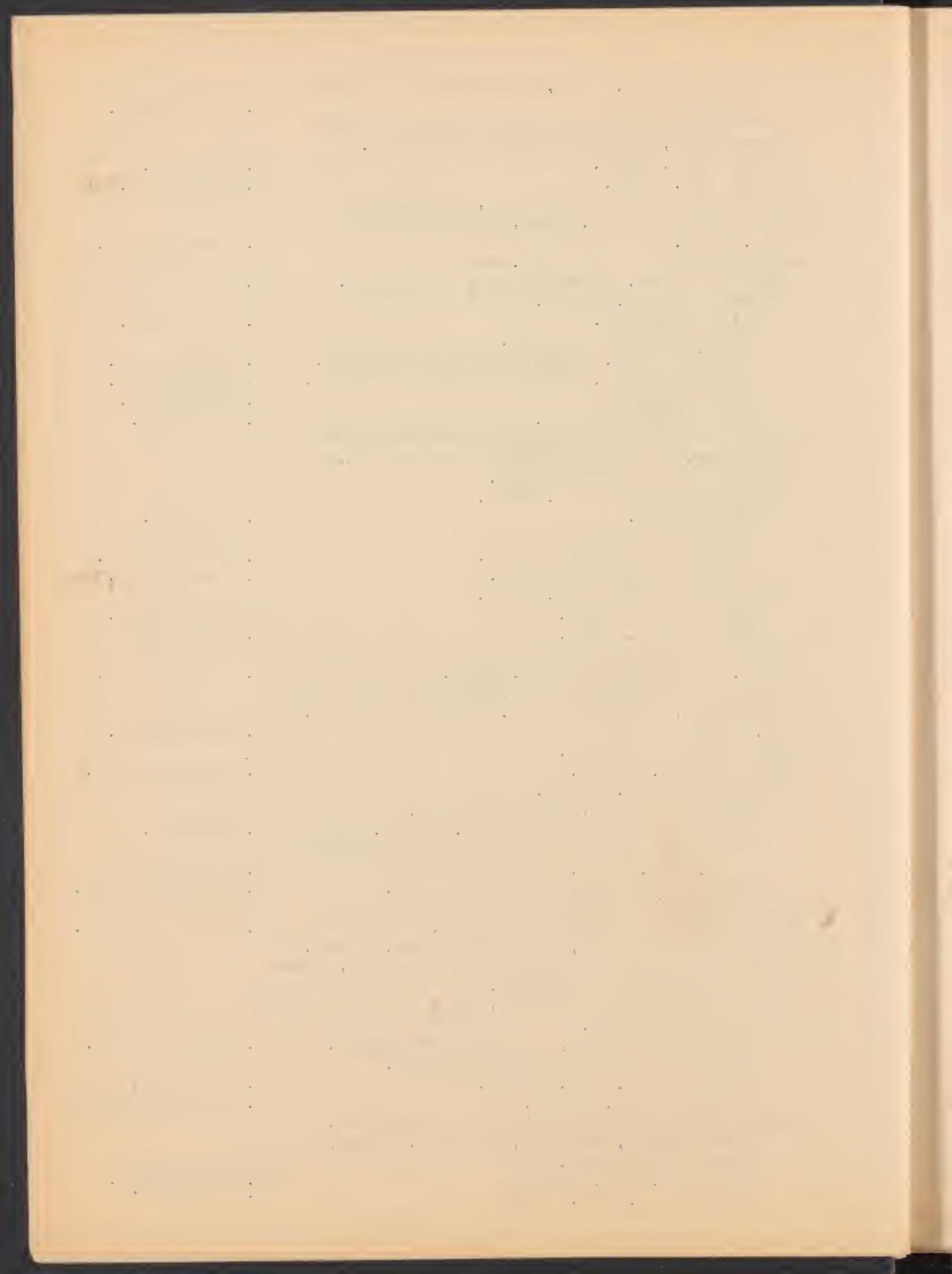


Pileus ponmex to subexpanded, not umbonate,

$0.5-2 \mathrm{~cm}$. broid; lamel Iae subcrodued.

Pileus consricous]y M. hinnuleiformis Surface of pileus white to plumbers sumbeous, umbi-

Iicate, $12 \mathrm{~mm}$.broad; stipe twisted, $6-8 \mathrm{~cm}$.Iong. M. tortives

Surface of pileus pale-ereen.

Surface of pjleus isabeline or fulvous, some-

M. viridifuscus.

times with Erayish tints.

Pileus $1-3 \mathrm{~cm}$. broad, rarely larger in M.mon-

tanuus.

Pileus convex, rarely fully expanding,umbo-

nate or Eibbous.

Stipe $3 \mathrm{~cm}$. Ione.

Stipe 4-8 cm. lone.

M. Harrisii.

Pileus Eibbous; iamellae crowded.

stipe erayish-pulverulent.

Pileus frominently umbonate; lamel-af not

Iae not crowded.

Lileus plane or depressed, never umbonate.

Stipe elabrous.

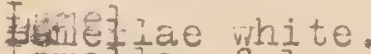

LameIJae fuIvous.

Stipe pruinose, becoming subelabrous

a.t times.

Stipe $5 \mathrm{~mm}$. thick; gecies terrestrialM. pruinosifolius

stipe 1-2 mm. thick; species not terrestrial.

Stipe densely velvety-fruinose. M. cervinicolor. stipe whitish-pulverulent to subelabrous.

Pileug 7-15 mm. broad.

Pileus $2-4 \mathrm{~cm}$. broad.

Pileus il.ane.

Pileus umbilicate or de-

Pileus $4-7 \mathrm{~cm}$. broad.

$$
\text { pressed. }
$$

Lamellae free; stipe $2.5 \mathrm{~cm}$. long

Lamellae adnate; stine $5 \mathrm{~cm}$. Long.

Surface of pileus bay, chestnut, redilis, or

Witredinis.

ferruejnous.

Lamel lae crowded and connected by conspisolu

cuous retculate veins.

Lamellae distant, nit conspicuously connected by veins.

Pileus ferrueinous, $4 \mathrm{~cm}$. broad.

Pileus baygorychestnut, not more

than $2 \mathrm{~cm}$. hroad.

Stipe pruinous to glabrous, $2.5 \mathrm{~cm}$. lone.

stipe elabrous and shinine from

M. bahamensis.

W. hemileucus.

M. montanus.

il. fibrosipes.

N. subpruinosus. the first, $3-6 \mathrm{~cm}$. Ions.

ii. polyporoides.

Lamellae about $12-16$ in number. M. haematocephalus

Mi. Berteroi.7302

m. 5028

M badius. 


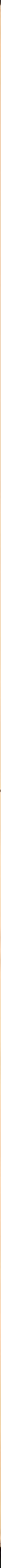


Lamellae about, 8 in number M. taceticolor. Surface of nileus some shade of purple or viol.et. Surface pale-purple, fading to whitish onridryine; lameliae decurrent;oileus infundibuliform.

Surface purple or violet, not fading to whitish on drying.

Pileus about $2 \mathrm{~cm}$. broad. Iamellae pallid.

Lamel.lae blackish-nurule.

Pileus 2-6 cm, hroad. surface of pijeus brown or somewhat M. puppurascens. hlackish.

Surface pale-fuscous; lamel lae adnexed; pileus convex

Surface brown, rarely tingted with violet.

Stipe less than $1 . \mathrm{cm}$. Ione. stipe $2 \mathrm{~cm}$. or more lone. pileus becoming distinct]y depressed; lamellae not crowded. Iamellae decurrent. Lamellae adnate.

Pileus convex to platilamellae

Pileus merioranous to somewhat ifeshs; Pileas

M. coracjnes.

M. sericipes.

N. Elaucorus.

ih. jonides. glabrous or nearly so: Iameziae adnate to

Pileus thin, reviving, conspicuosly decorated

with threadlike hairs, scales, or $\mathrm{s}_{1}$ ines;

lameljae adnate or adnexed;spores hyraline;

veil none; stipe central, ilender, tubular.

Pileus $1-2.5 \mathrm{~cm}$ broad.

Pijeus less than $1 \mathrm{~cm}$. broad.

Pileus papillate or umbonate.

2ileus fulvous.

Stipe 1-2 cm.

Pileus not as above.

Surface villose.

Stipe $4 \mathrm{~cm}$.

Surface echunus.ate

stipe solid; thjck;uniform texture. Veil

M. subcyathiformis.

M. Johnstonii.

M. curtipes.

M. cya,thiformis.

Crinipellis 287

C. sublivida, $\mathrm{M}$.

aboent. Hymenophore soljtary or eregarjous.

Pileus recular $\mathbf{9}$ Pijeus thin umbijicate. Lameliae

decurrent.

Pileis glabrous

Lentinus,

Pileus white or whitishiless than $1 \mathrm{~cm}$.I. orizabensjs.

Pileus isabelline

henfoulty $\mid \begin{aligned} & \text { Hymenophore solitary } \\ & \text { spores } 7.31 .4 \mathrm{At} \\ & \text { Hymenomhore Evegarious } \\ & \text { Spores } 3.5 \text { if } 2.5\end{aligned}$

Pileus fulginous

c. stupparia.

Pileus reddish-brown

Pileus $1-1.5 \mathrm{~cm}$.

L. eraminicola. 7312

I. subscy phoides. 7236

I. firleginells.

I. scyphoides. 

Pileus $6-8 \mathrm{~cm}$ diameter

Pileus Bcaly, imbricate

Pileus $3-5 \mathrm{~cm}$, white or yellowish with concen-

tric pyamidal fascicles

Pileus $5-8 \mathrm{~cm}$, white with brown maculiform

scales

Pileus velvety, strigose or hispid

Pileus $2-7 \mathrm{~cm}$

Stipe glabrous or nearly so

Stipe velvety or tomentose

Pileus isabelline, stipe usually excentric

Pileus dark fulvous

Pileus pale cinnamon to chestnut brown, stipe slender, concolorous

Pileus 8-15cm; stipe $1-2.5 \mathrm{~cm}$.

Pileus white, spores narrow ellipsoid,

$$
6-8 \times 3-3.5 u
$$

Pileus isabelline, spores oblong ellipsoid or fusiform, $4-5 \times 1.5-2 u$
Lentinus tubarius

L. pyramidatus

L. albellus

L. crinitus

L. strigosus

L. strigosellus

L. velutinus

L. hirtus

L. hirtiformis

See also:

L. chaetoloma and Panus Troglodytes, P. reflexa related to L. crinitus

L. flaccidus, L. fuligineus, L. furfurosus, L. glabratus, L. xylopodius and Panus connatus, related to L. hirtus

Pileus thick, convex; gills adnate

Veil present; hymenophores densely cespitose, surface smooth
Lentinula detonsa (Fr.)Murr.

Lentodiellum concarum (Berk)Murr.

Hymenophore fleshy or membranous

Stipe excentric, lateral or wanting

Pileus sessile, often partly resupinate when young see also P. limpidus

Pleurotus (segregated

Pileus less than lcm, rarely more in G.cupulatum

Pileus resupinate

Pileus $1-2 \mathrm{~mm}$

Pileus 3-8m

Pileus not normally resupinate

Surface white,gills whitish, pruinose on edges

Surface pale ochraceous; gills not whitish pruinose on edges

Pileus more than $1 \mathrm{~cm}$ broad surface ferruginous

Surface translucent, glabrous, pileus reniform

Surface milk white

Pubescent, pileus flabelliform

Viscid, thin, pileus dimidiate

Surface isabelline, pileus $6 \times 9 \mathrm{~cm}$

Surface orient pink, nileus $33 \mathrm{~cm}$.

Surface buffy brown or lighter, $11 \times 8 \mathrm{~cm}$

Surface salmon buff, pileus $12 \times 20 \mathrm{~cm}$, crisped

Geopetalum

Surface white to pale fuscous, spongy pubescent to

glabrous; pileus flabelliform or spatulate

Surfece sordid grayish olive, spongy tomentose, gills zinc orange to tazny

Surface fuscous, spongy tomentose, gills yellozish

Surface avellaneous, spongy tomentose, $3.5 \mathrm{~cm}$.,

gills white

G. inconspicuum

G. subelatinum

G. haedinum

G. copulatum

G. tremelliforme

G. catephes

G. subhoedinum

G. Siquirres

G. 7647

G. Cattii

G. 5058

G. 4806

G. semitectum

G. serotinoides, 6746

G. flavolanatum

G. Barnstorf, 5510, 7317

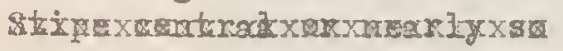




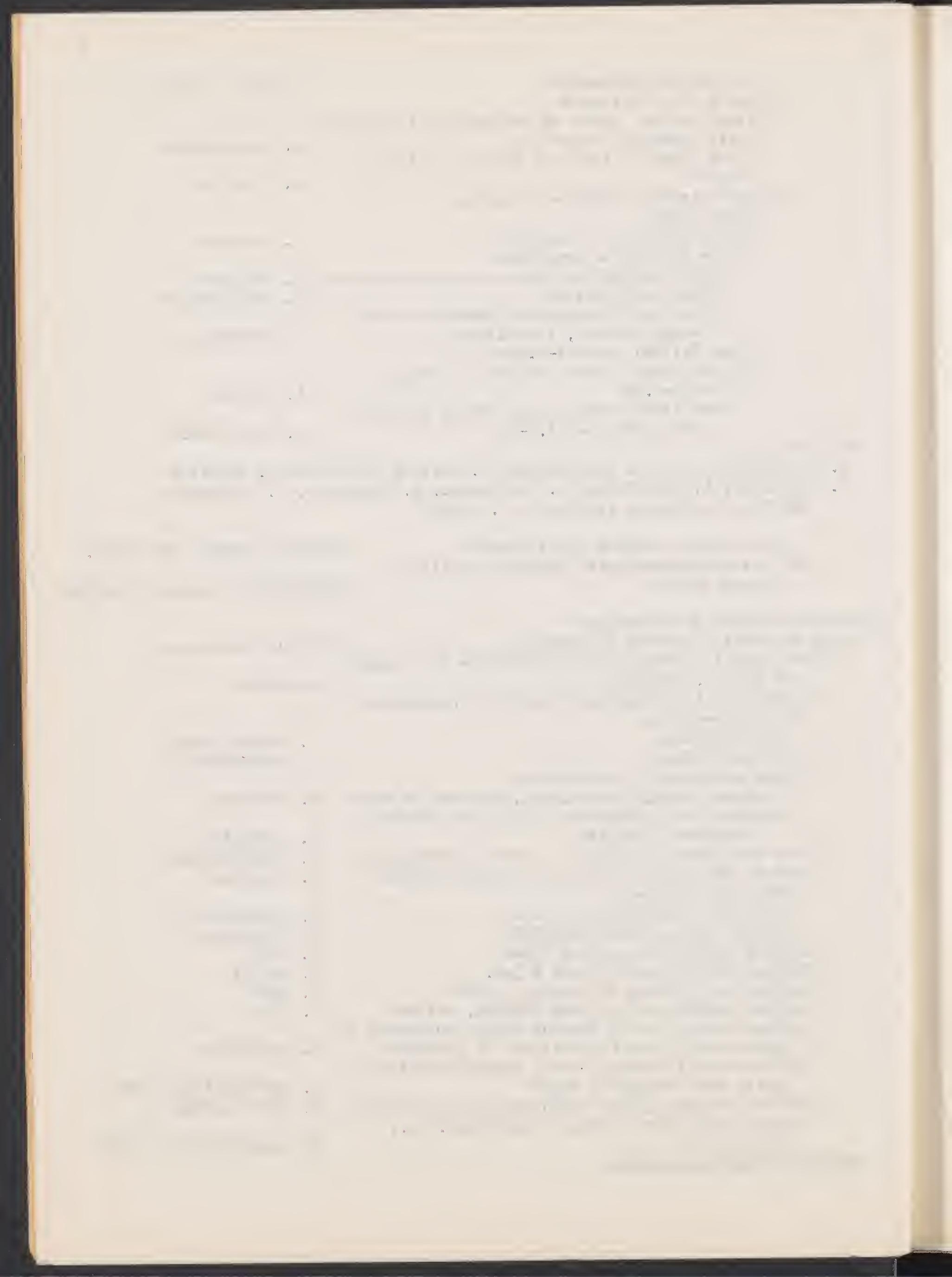


Pileus stipitate (laterally)

Gills conspicuously decurrent

Pileus drying reddish

Gills crowded, nar row, stipe $2 \mathrm{~cm}$. long

Gills very distant, interveined, stipe very short

Pileus white or whitish,yellowing on drying

Pileus $11 \times 14 \mathrm{~cm}$; stipe $2 \times 1 \mathrm{~cm}$

Margin striate,gills narrow

Margin not striate, gills broad

Pilous $4-6 \mathrm{~cm}$ broad, stipe 1-5 $\mathrm{rm}$

Gills narrow, crowded, stipe $4-5 \mathrm{~cm}$ long

Gills rather broad, subdistant

Stipe glabrous, $1-2 \mathrm{~cm}$ long

Stipe tomentose, $2.5-5 \mathrm{~cm}$ long

Pileus $2-4 \mathrm{~cm}$ broad; stipe $4-8 \mathrm{~mm}$

Pileus orient pink, $3 \mathrm{~cm}$

Gills adnate or sinuate

Surface conspicuously fibrillose

Surface glabrous or pulverulent

Surface white or nearly so

Margin striate, pileus $7 \mathrm{~cm}$, gills subdistant, broad, stipe $5 \times 1 \mathrm{~mm}$

Margin not striate, gills close to crozded

Pileus 18mm, gills crowded, narrow, stem $12 \times 2 \mathrm{~mm}$

Pileus $4 \times 3.2 \mathrm{~cm}$, gills close, broader, stem $35 \times 4 \mathrm{~mm}$

pileus $7 \times 10 \mathrm{~cm}$, margin crisped, stipe $7-8 \times 5-6 \mathrm{~mm}$

Surface bay

Surface pale fuscous, gills narrow

Surface drab to cartridge buff

Surface fuscous to blackish, gills broad

Surface Verona brown to cartridge buff, gills

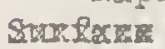

Naples yellow

Surface viscid, Dresden brown to cartridge buff

Stipe central

Stipe slender, tubular with cartilaginous cortex

Margin at first straight and appressed to stipe

Pileus plicate, sixsplitting down backs of lamellae

Stipe conic, $15 \mathrm{~mm}$ at base, $4 \mathrm{~mm}$ above

Stipe equal

Pileus less than $1 \mathrm{~cm}$ broad

Pileus $2 \mathrm{~cm}$ or more broad

Gills adnexed

Margin ciliate

Margin crenulate

Gills free

Pileus plane, pale purple

Pileus campanulate, $r$ eddish brown

Pileus even or striate, not splitting dow backs of gills

Pileus unbilicate; gills decurrent

Pileus entirely smoky white, gills very distant

Pileus white or whitish, differently colored on disc

Disc reddish brown; stipe scarcely $1 \mathrm{~mm}$ diam.

Disc pale yellowish, solitary; stipe 2-3rm

Disc subfuscous, densely cespitose, stipe 2-3mm

Pileus pale vellaneous, $8 \mathrm{~mm}$ broad;gregarious

Pileus some shade of yellow or orange see next $p$.

Crepidopus

C. 5382

C. hemiphlebius

C. 7250,7726

c. 5369

C. caveatus

C. commiscibilis

c. connatus 7669?

C. Eugeniae, 7324,7289, 7252, 5 coll.Golfo Dulce

C.? Cattii

Microphiale

M.fulvifibrillosum

M. 5383

M. excavatum

N. Carpenteria, 6238

M. 4796

M. badium

M. brunnescens

M. 5220

M. fuscifrons

M. 5365

M. viscidus, 7695

Hiatula

H. discreta

H. minima

H. ciliatulus

H. crenulatus

H. purpurascens

H. Benzonii

onphalia

o. distantifolia

0. convexa

O. petasiformis

0 . euspeir ea

O. subavellanea 


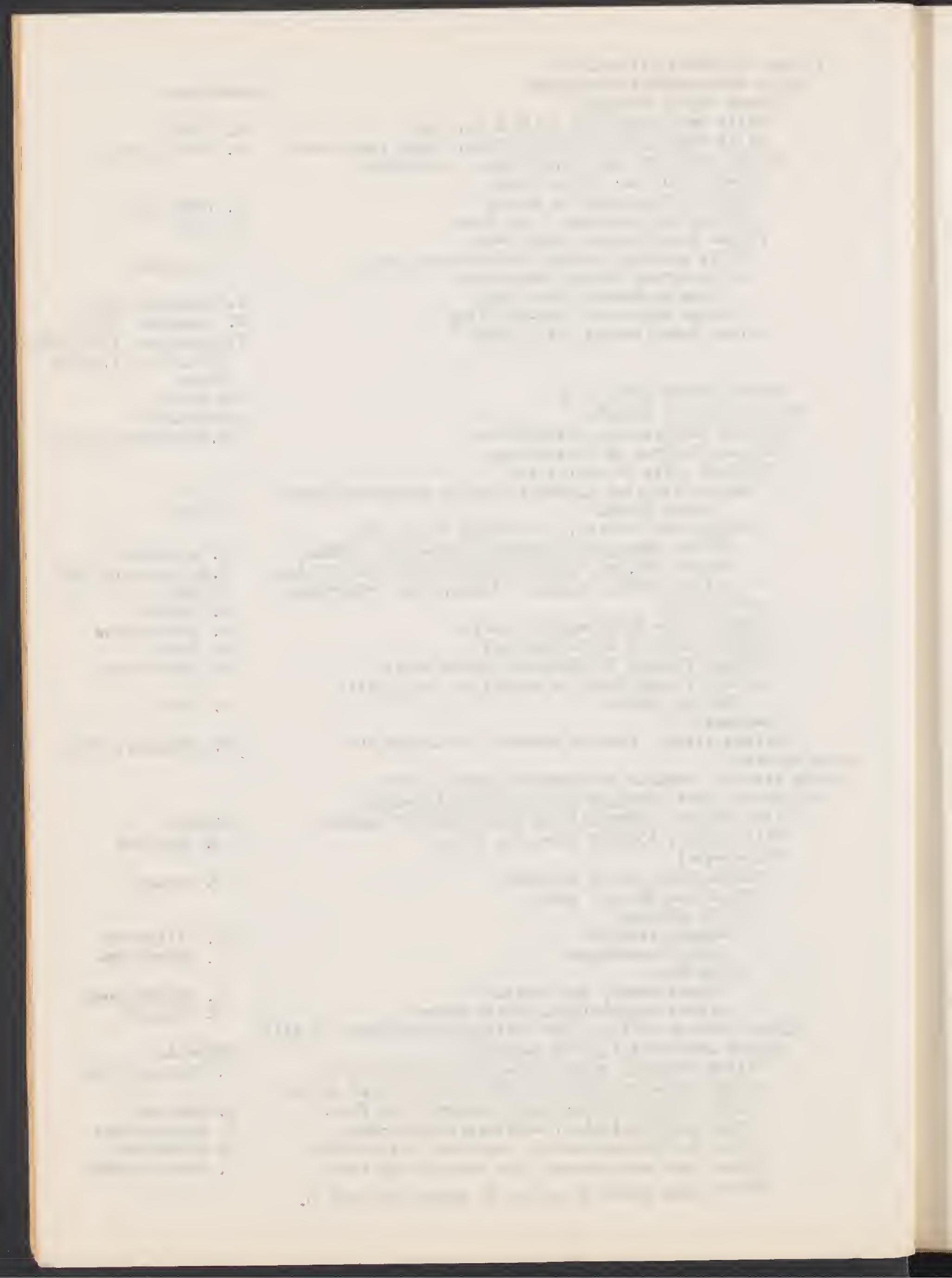


Pileus some shade of yellow or orange

Hymenophbres solitary or gregarious

Pileus lemon-yellow, $5 \mathrm{~cm}$.broad, among dead leaves 0 . citricolor

Pileus orange yellow, often fading, $2-13 \mathrm{~mm}$, among mosses

Pileus stramineous with grayish tint, $12 \mathrm{~mm}$. broad

Pileus dull rosy-isabelline, $2 \mathrm{~cm}$ broad

0. fibula Hymenophores cespitose

Pileus pale isabelline; stipe glabrous

Pileus yellowish ferruginous to dull reddish

yellow, stipe with brown hairs below

Pileus pale avellaneous, $8 \mathrm{~mm}$. broad,gregarious

Pileus convex to umbonate

Gills free

Gills adnate to adnexed

Pileus white, whitish or cinereous, sometimes

differently colored on the disk

Surface entirely white (see also M. pruinosus (pileus farinose)

Pileus $1-4 \mathrm{~mm}$ broad

Stipe 4-5m long

Stipe $1-2.5 \mathrm{~cm}$. long

Pileus $2 \mathrm{~mm}$, striate

Pileus 2mm, not striate

Pileus 4m, striate

Pileus 4mm, not striate

Pileus $1.5-2 \mathrm{~cm}$ broad

Surface white with fuscous umbo

Surface sinereous with pale avellaneous umbo Pilous and gills greenish gray

Pileus yellowish avellaneous or brownish

Pileus 4-8rm broad

Stipe $1 \mathrm{~cm}$ long

Stipe $2 \mathrm{~cm}$ long, avellaneous

Pileus solitary or gregarious, striate

Surface pale avellaneous, ashy white on disc

cf. M. 5341

5090

5096
Surface avellaneous with smoky tint, dise concolor

Pileus cespitose, not striate

Stipe 5-7cm long

Pileus about $1 \mathrm{crn}$ broad

Surface Naples yellow, umbonate, not striate stipe $2 \mathrm{~cm}$

Surface avellaneous, striate

Surface dark brown, not striate

Pileus $1,5-2 \mathrm{~cm}$ broad

Pale avellaneous or isabelline

Sayal browm

Olive brown

Brussel br ow

Exymxt

Pil eus $3 \mathrm{~cm}$. broad

Avellaneous

Buffy brown shading pale, umbonate

Sayal brown

Tawny to russet
M. Myrciae, 7259

M. 7280

M. alphitophorus

M. subpulverul entus

M. 7270

M. trojanus, 72973 7240,7649

M. roridulus, 7319

M. Margarita

M. viridigrisea, 7203 ?

M. Siquirres Dec. 5 cf.7299
M. argillaceus, 7239

M. pubescens, 71,87

M. cinchonensis, 7309 7295, 7655

M. 5112

M. 5113,7664

M. 4656

M. 5361

M. 5126

M. 5127

M. 5106 


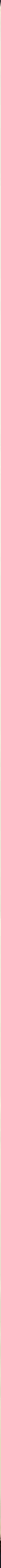


Pileus $5 \mathrm{crn}$, wood brom

Pileus $2.2 \mathrm{~cm}$ broad, wood brown Hazel

Pileus lilac or some shade of reddish

Pileus 5-8ma broad

Lilac

Dark testaceous

Pileus $1-2 \mathrm{~cm}$ broad, stipe $2-3 \mathrm{crn}$ long

Latericeous, gills testaceous

Chestnut $r e d$; gills fazn color

Madder brown to pompeian red

Pileus 2-5crn; stipe 5-8cm; rose lilac

Margin at first incurved

Gills decurrent; pileus urabilicate

Pileus white or whitish

Pileus under $13 \mathrm{~mm}$ broad

Pileus $5 \mathrm{~mm}$ broad, stipe $6 \mathrm{~mm}$ long

Pileus 8-13nn, stipe $1-4 \mathrm{~cm}$

Pileus not unonate

Stipe $2 \mathrm{~cm}$, pileus $13 \mathrm{~mm}$

Stipe $3 \mathrm{~cm}$, pileus $10 \mathrm{~mm}$

Stipe $4 \mathrm{~cm}$, pileus $8 \mathrm{~mm}$

Pileus umbongte

Pileus convex with abrupt small umbo

Pileus conic with a long pointed umbo

Pileus $3 \mathrm{~cm}$ or more brosd

Pileus convex to umbilicate, gills distant

Pileus infundibuliforra; gills crowded

Pileus yellow or yellowish

Pileus infundibuliform

Pileus $1-1.5 \mathrm{~cm}$ broad

Isabelline

Flevovirens becoming melleous

Medal bronze to citrine

Pileus $3-4 \mathrm{~cm}$, isabelline may be only large form of 0 . lents

Pileus $3 \mathrm{~cm}$ drab, reticulated darker

Pileus convex, $2-3 \mathrm{~cm}$ broad

Stipe $1 \mathrm{~mm}$ in diameter

Stipe $2-4 \mathrm{~mm}$ in diameter

Pileus umber (if raw cf. spec. from Castilla Jy23

Pileus $r$ ed or $r$ eddish

Pileus $6 \mathrm{~mm}$ broad, gills distant

Pileus $12 \mathrm{~mm}$ broad, gills crozded

Pileus $2.5 \mathrm{~cm}$ broad

Gills adnate or adnexed

Pileus glabrous

Pileus not viscid

Pileus entirely white or whitish

Pileus $1-2 \mathrm{~cm}$ broad, gills adnexed crowded, narrow

Pileus $3-8 \mathrm{~cm}$ broad

Gills deeply sinuate, narrow, crowded

Gills adnate, broad

Pileus whitish, differently colored on disc

Disc pale yellowish; stipe $1.5 \mathrm{~cm}$
M. 5375

M. 5379

M. 7660

M. syringeus

M. testaceus

M. latericius

M. corrugatus

M. 5346

M. purus

Onphaline

0. niveicolor, 7193 , $7293,7221,7205$

O. subscyphoides

Q. Golfo Dulce 7710

O. tepeitensis

0. cuspidatel la

o. acuminata

0. collybiiformis, 7644

o. jalapensis

0. Ienta, 7680,7734

0. flavella, 7328,726 771

0.7217

0. Sandalo 7712

o. merulioides n. sp.

0. Earlei

O. cr emea

0 . chondripes

0. miniata

0. coccinea

o. carneola

Collybia

c. muscicola

C. Boryana

C. albidula

c. orizabensis 


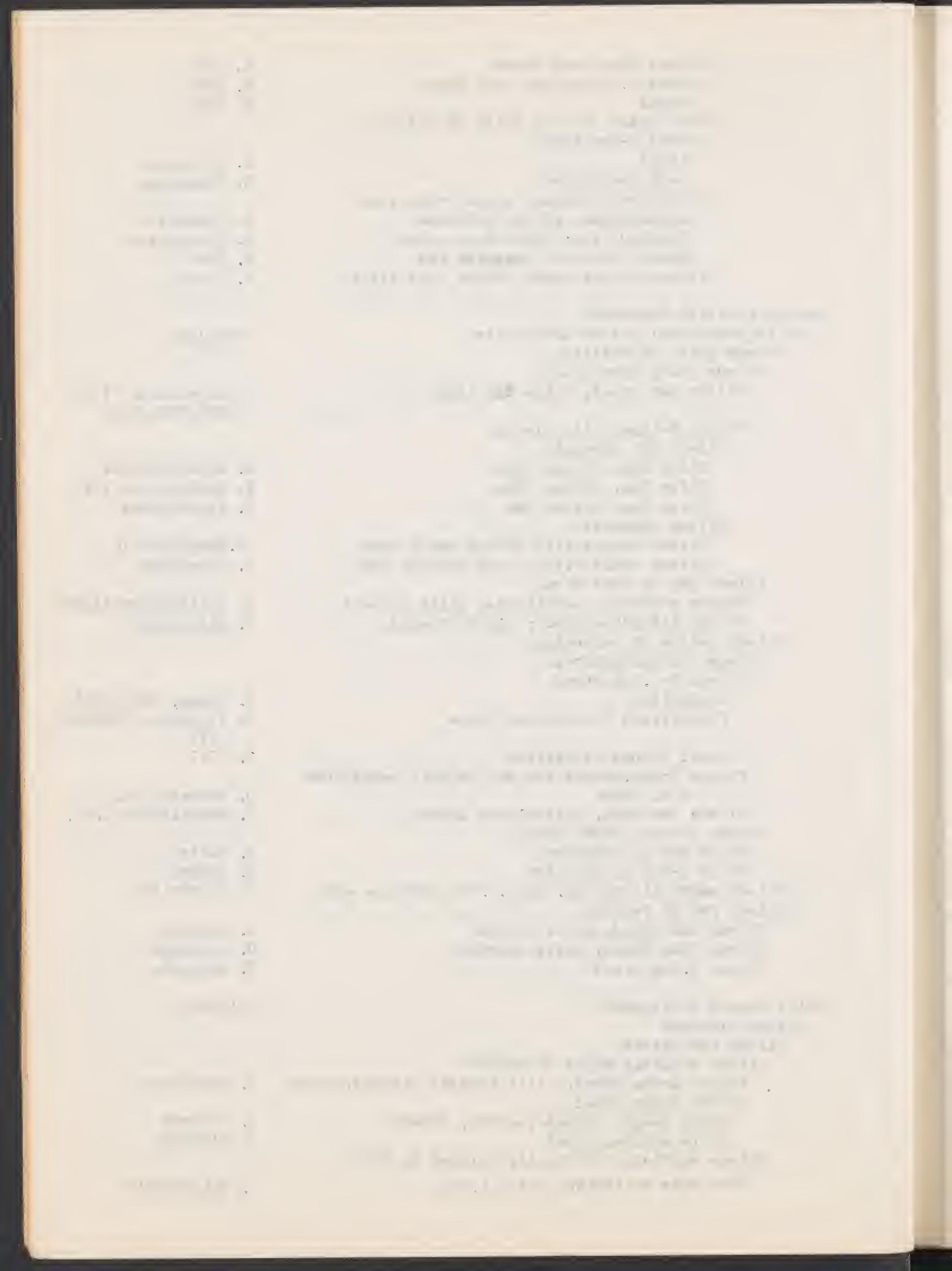


Disc tasny

Disc mummy brown

Disc pale olive buff

Disc russet; stipe $4 \mathrm{~cm}$; gills broad

Disc latericious; stipe $6 \mathrm{~cm}$; gills narro:

Pileus ochraceous, isabelline or fulvous [cf also

Pileus pale ochraceous, 5-10mm broad

Pileus pale ochraceous salmon with russet umbo,

$5 \mathrm{~cm}$ broad, margin striate

Pileus dark ochraceous, 1-3cm, margin even

Pileus isabeline with pale latericious unbo

$5 \mathrm{~cm}$ broad,margin splitting

$P$ ileus ochraceous tany with cinnamon brown umbo

Pileus antimony yellow with hazel disc, $5 \mathrm{~cm}$, margins rugose striate

Pileus fulvous

Gilis white

Stipe $2-3 \mathrm{~cm}$ long, pileus becoming fuliginous on drying

Stipe $1 \mathrm{~cm}$ long; pileus remaining fulvous

Gills brown

Pileus wholly cinnamon rufous with large umbo, gills nearly free

Pileus cinnamon rufous, disc chestnut

Pileus russet, $5 \mathrm{~cm}$ diam

Pileus avellaneous

Gills white

Pileus pale avellaneous [tilleul buff?]

Pileus wood brown with fawn disc

Pileus reddish brown

Gills pale murine

Pileus rosy livid, $12 \mathrm{~mm}$ broad

Pileus black or nearly so, at least on drying

Gills blackening on drying

Gills remaining white on drying

Pileus orange, yellozish or brownish

Pileus convex, umbonate, $2-3.5 \mathrm{~cm}$ broad

Umbo large, margin upturned, slightly lacerate, pileus cinnamon

Umbo smaller and les prominent,margin not upturned, striate to sulcate

Gills narrow, white; stipe $7-8 \mathrm{~cm}$ long, 1.5-3 $\mathrm{mm}$ Pileus pale ochraceous salmon, innate fibrillose, gills adnate

Pileus honey yellow; gills adnexed

Gills medium, distant, tilleul buff

Gills broad, adnate

Pileus pale ochraceous salmon with russet umbo

Pileus vinaceous fawn, hygrophanous

Pileus companulate to subconic, gills adnate

Gills broad, subdistant, mustard yellow, pileus munmy brown to buckthorn brown, stipe velvety rar umber below to raw siena above;margin striate

C. 814

c. 7732
C. 4427,7668 ?

C. Sept. 8

C. Nevermann

C. 4810

C. oculatus, 7219?

C. dryophila]

C. marasmitormis

C. 4810

C. domesticus

axx 2826

c. monticola

C. 4996

C. 7281
C. nigritiforms

C. densifolia

C. fimetarius

C. Sandalo 7732

c. 7253

C. 5387

C. cinchonensis 7696

C. 7213

C. subnivulosus

C. subavellaneus

C. roseilivida

C. nigrtia

C. irrorata

C. nigritiformis

gills narrow, close; pileus hygrophanous 

Gills narrow, close; pileus hygrophanous

Pilous pinkish buff to mars brozn, gills pinkish cinnamon, stipe white

Pileus cinnamon brown to ochraceous tawny, gills light ochraceous buff, stipe Hessian brown, lower quarter clothed with whitish tomentum

C. 710

Pileus hygr ophanous

Laccaria

Pileus umbonate, light pinkish cinnamon to Sayal brown

Pileus $6 \mathrm{~cm}$, stipe $9 \times 0.6 \mathrm{~cm}$

Pileus $1.5-1.8 \mathrm{~cm}$, stipe $2 \times 0.1 \mathrm{~cm}$

Pileus umbilicate

Pileus pinkish buff, $8 \mathrm{~m}$; stipe $10 \times 1 \mathrm{~cm}$

Pileus dark livid brown to livid brown, $3 \mathrm{~cm}$; stipe $4 \times 0.5 \mathrm{~cm}$

Pileus salmon buff, $1 \mathrm{~cm}$; stipe $2.5 \times 2-1 \mathrm{~cm}$

Pileus expanded to flat or even depressed but not umbonate or umbilicate

Pileus fawn, disc army browmargin, $4 \mathrm{~cm}$; stipe white $2 \times 0.25 \mathrm{~cm}$

Cinnamon disc, bister margin, $2.3 \mathrm{~cm}$; stipe wood brown, $4.5 \times 0.2 \mathrm{~cm}$

Pileus deep brown drab, $1.5 \mathrm{~cm}$; stipe cinnamon $\mathrm{drab}, 3 \mathrm{x} 0.25 \mathrm{~cm}$

Pileus buff pink to vinaceous tamy; $3 \mathrm{~cm}$, stipe onionskin pink, $2 \times 0.2 \mathrm{~cm}$

Pileus hazel, $0.9 \mathrm{~cm}$; stipe light russet vinaceous $8+2 \times 0.2-0.4 \mathrm{~cm}$

Pileus viscic

Pileus white

Pileus tamy or reddish yellow, cespitose

Pileus chestnut, solitary

Pileus Saccardo's umber

Pileus with hairs or scales

Pileus white with floccose, subseparable pellicle

Pileus orange, densely furfuraceous velvety

Margin not striate; gills crovded; stipe $3 \times 0.4 \mathrm{~cm}$

Margin striate; gills distant; stipe $3.5-5 \times 0.2 \mathrm{~cm}$

pileus ochraceous to yellowish red becoming bay or chestnut, densely tomentose zhen young

Pileus mahogany rod with bay or chestnut umbo

Pileus beckthorn brown on warm buff background

Pileus buffy brown.

Pileus black:

cf. 7671,7690

stipe stout, fleshy, of uniform texture

Context of vesiculose cells

Very watery or milky juice present

Pileus viscid, tamingmilk watery becoming

testaceous and blackening

Pileus velvety

Flesh buff yellow on wounding

Flesh buckthorn brown, tarmy

Pileus fibrillose scaly, warm sepia

Pileus moist, cinnamon rufous to light ochraceous buff

No watery or milky juice present, usually dry, light

Pileus rough, fibrillose velvety or floccose, gills close,narroz, taste mild
L. 7646

L. 6133

L. 6134

L. 5153

L. Aug. 7
L. 5364

L. 5155

L 5154

L. July 13

L. JuIy 16

Collybia

C. Sandalo 7716

C. velutipes

C. July 11

C. 5062

C. subflavescens

C. 5128 , cf. Aug. 29

C. chrysopeplus

c. tenuipes, 7311,7263 , $7238,7677,7687$

C. 5028

C. Aug. 21

C. 5368

c. 5131

Lactarius

L. july 10

L. Aug. 7

L. 4993

L. July 7

L. 6129

Russula 


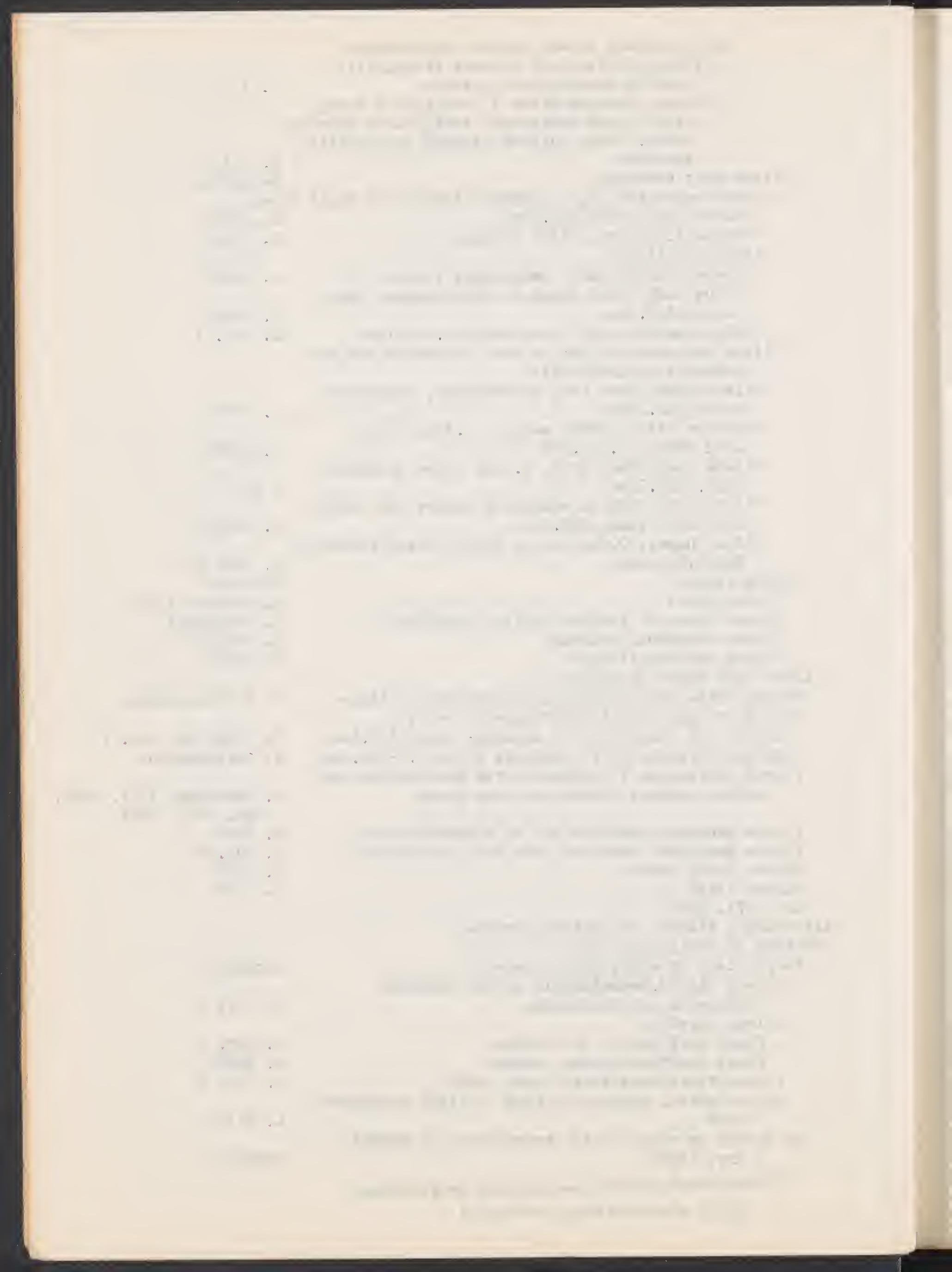


Pileus rough, fibrillose velvety or floccose, gills

close, narrow, taste mild

Pileus dirty white, $7-8 \mathrm{~cm}$, fibrillose; stipe white R. 5034

Pileus alizarin pink to pale rose; $3 \mathrm{~cm}$, velvety,

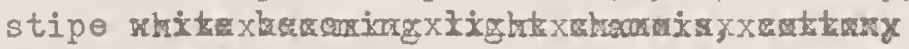
tureatase warm buff

Pileus isabelline, $3 \mathrm{~cm}$, floccose verrucose; stipe White becoming light chamois, tottony tomentose R. 5247

R. 5150

Pileus viscid even when old

Pileus purplish, pellicle separable; spores wite; taste mild; stipe white

Margin striate; gills broad

Margin not striate; gills very narrow

Pileus cream color with old gold disc; pellicle not separable; stipe white;margin striate sulcate

Pileus buckthorn brown; pellicle separable; stipe white, margin rugose striate sulcate; gills cream color

Pileus $5.5 \mathrm{~cm}$; stipe $2.5-4 \times 2 \times 70.8-20 \times 1.0 \mathrm{~cm}$

Pileus $1.7 \mathrm{~cm}$; stipe $2.3 \times 0.4 \mathrm{~cm}$

Pileus mars br own shading to hazel and russet on striate margin, $4 \mathrm{~cm}$; stipe $35 \times 6-8 \mathrm{~mm}$, pale ochraceous salmon

Pileus Dresden brown to mumy brown, $2.7 \mathrm{~cm}$., pellicle not separable; gills cresm color

Pileus bister, pellicle separable, margin not striate; stipe zhite

Pileus cinnamon buff shading to zhite, pellicle not separable;stipe white, margin striate

Pileus dry, at least when old

Pileus probably viscid when young; margin smooth or only faintly str iate

Pileus isabelline, $3.5 \mathrm{~cm}$; stipe $3 \times 1-1.5 \mathrm{~cm}$, white staining brownish

Pileus deeply vinaceous lavender, $4.5 \mathrm{~cm}$; stipe $2.4 \times 0.8 \mathrm{~cm}$

Pileus brownish, $6-7 \mathrm{~cm}$

Liver brow with donnamon buff or clay color disc; stipe white, $2.5 \times 0.7-0.9 \mathrm{~cm}$

R. 5033

R. 5032

R. 5148,5147

R. 5149, July 7

R. 5145

R. 5144

R. Zurqui 6130

R. Aug. 14

R. July 7

Diamine brown, maculate lighter and darker; stipe white fibrous, $2 \times 1.6 \mathrm{~cm}$.

Light russet vinaceous to Hays brown and vinaceous tazny; stipe light pinkish lilac, $5 \mathrm{xl} .5 \mathrm{~cm}$

Pileus variegated sayal brown and bister, $9 \mathrm{~cm}$; stipe silky fibrillose, $8 \times 2.5 \mathrm{~cm}$ white

R. July 11

R. 5238

R. 6629

R. 5404

R. 5035

R. July 7

Pileus wholly dry; pelicle not easily separable Pileus black, margin smooth

Pileus rimose areolate on disc, $18 \mathrm{~cm}$ diam.; gills white with dark edges, derkening; stipe $5.5 \mathrm{~cm}$ diemeter

Pileus smooth, $8 \mathrm{~cm}$; gills cream becoming dark gray; stipe 1.6 ma diameter

Pileus Prouts brown,margin striate;gills broad; stipe ventricose to subfiusiform, $2.5 \times 0.4-0.75 \mathrm{~cm}$, flesh thin, taste slorly acrid

R. 5146

Pileus warm buff on disc, etc. see next p. 

Pileus warm buff on disc, rest maculate of buff vinaceous and drab; gills narrow; stipe tapering upward, $5.5 \times 0.4-1.5 \mathrm{~cm}$; flesh thick, taste mild; margin not striate

Pileus Etruscan red mottled buff pink; gills broad antimony yellow; stipe $2 \times 0.7 \mathrm{~cm}$; flesh thin, taste mild; margin not striate

Pileus hazel, mottled lighter shading to seashell pink; margin sulcate striate; gills white; stipe whtte, $4 \times 0.8-1.2 \mathrm{~cm}$, ventricose

Context without vericular cells in trama Gills waxy

Pileus viscid; bright colored fragile

Pileus small, less than $1.5 \mathrm{~cm}$ in diameter

Stipe $1.5-2.6 \mathrm{~cm}$ long

Gills decurrent

Gills adnate or adnexed

Pileus luteous with faint traces of red; Stipe citrine

Pileus and stipe apricot yellow

Pileus and stipe orange

Pileus Brazil red with orange chrome disc

Stipe 3-4cm long; gills decurrent

Pileus yellow

Pileus red

\section{Pileus forruginous}

Pileus medium to large, usually $2-5 \mathrm{~cm}$ broad, rarely smaller in $\mathrm{H} . \mathrm{la}_{\mathrm{e}} \mathrm{ta}$ and $\mathrm{H}$. flammea

Pileus distinctly umbonate

Pileus blackening on drying

Pileus not blackening

Pileus convex to plane or depressed

Pileus ple reddish yellow; stip e pale yellow; spores sphericel

R. July 11

Hygrophorus (segregated)

Hydrocybe

H. rosea

H. flavolutea

H. 5237

H. aurantia

H. 5139

H. hondur ensis

H. subminiata

H. 4460

H. trojana

Plleus and stipe strontian yellow to yellowish citrine, $6 \mathrm{~cm}$

Pileus greenish black, $3.5 \mathrm{~cm}$

Pileus ruber to miniatus; stipe luteous or paler

Pileus incarnate, not fading, rarely varying to white or pale fuliginous; stipe fulvous

H. conica

H. subflavida
H. Earlei

H. 5024

H. 5366

H. subcrespitosa

H. laeta 

Pileus Beconia rose, minutely fihrillose; sti fe coral ink shadingto flesh ochre below H. 6022

Dileus scarlet, soon fading
siores $8-9 \times 4-6 u$
siores $18 x 10$ u
H. flammea
H. hella

Pileus not tiscid; not brjehtly colored, firm pileus white with a very lare umbo

Pileus gray

Pileus oranee, derressed at center

Pileus sepia to cinnamon buff

pileus cinnamon brown

Pileus olive ochre, depressed at conter

Camarophyllus

C. a. looumbonatus

C.?variolosus

C. Cantharellus

C. 5348

C. 5023

C. 4475

c. 7186

Glutinous, veil wesent forming an annulus or reduced to suluamules; pileus not brifhtly colored Hyerolnorus

Pileus vale fuscous when youne becomine paler except on disc

Puleno naw umber broad

Pileus rchracemontawny to rchar buff

Pileus sepia to isabelline

H. subjratensis

H. 4474

H. 5227,7196

H. 5137

Dileus stramineous to isabelj.ine with a testaceous tint $2.5 \mathrm{crn}$ broad

pileus dark chestnut brown

gels not wavilens white with floccose avellameous patches

H. montcinus

H. 5138

H. 7331

Volvaly and veil absent

Gills decurrent

Pile eus $5 n$ Q

Pile us $2,2 \mathrm{~mm}$ hroad comver umbilicate

Pileus $n \mathrm{mrn}$ broad.

Clitocybe

Pileus whitish to pale jasbelline

Pileus $1 \mathrm{~cm}$ broad

c. 9428

C. niveicolor

Pileus 2.5-3 cm; eills cream color; densely cespitose

Pileus $5-8 \mathrm{~cm}$ broad

Stije, 2-4 mm diam

stipe $4.6 \mathrm{~mm}$, gregarious, often excentric Pleurotus commiscibilis
Stipe $10 \mathrm{~mm}$ diam
Stilie $20 \mathrm{~mm}$ diam
C. 5373
C. mexicana

pileus pallid with a rosy tint, liuht bay on conic umbo
$2 \mathrm{~cm}$ broad
c.incrustata

Pileus dilute testaceous, deeply umbilicate, 3-5 cm

Filens range unfous to \&anford biown - ang 27

Dileus antimony yellow

C.testaceofle.va? 7218

Dileus clay color

C. 5100

Di I lis inkj.sh cinnamon with srya.I brown unbo

Gills sinutie

Pilens vimaceons ff rufus

Gills reddish; pileus latericious

dpores clobose, 3-4 u

spores ovoid, $9-12 \times 4-7 u$

Gills white, pale yellow or dirty brownjsh

C. 5020

pileus white with

तisc, viscid with remains of veil

C. 5266

Trichbjoma

T. jamaicensis

T. holoporihyra

T. 5514 


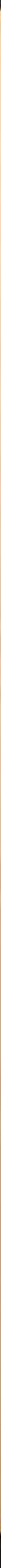


Pileus mukadobrown tomentose rumane areolate

$17929 a$

Pilens hqut ochraceves calmon tomentores strigose.

J. $87^{29}$

Pileus dingy isabeliine

Pileus and stipe purilish Jilac

Pileus chestnut, stipe white

Pileusdrying stipedrang

Tricholuma subisabellina

T. dichropus

T.jalapensis

7.7124

Tolva absent and veil present

Gills adnate or adnexed

Armillaria

Pileus viscid

Marcin striate, russet; stipe $5.5 \times 2-1.5 \mathrm{~cm}$ A. 5133

Naryin not striate, deep red or chocolate; stive $5-6 \times 3-5 \mathrm{~mm}$ Guadeloupe

pileus not viscid

A. umbjlilicata

Pileus areolate; pinkish cinnamon to Sayal brown $5 \mathrm{~cm}$ diam; stipe $3.5 \mathrm{~cm} x 7-11 \mathrm{~mm}$

A. 5257

Dileus suruemulose or verrucose, at least on disc

Margin striate

Pileus $5 \mathrm{~cm}$, white or jink? umbonate; stipe $2 \mathrm{~cm} \times 5 \mathrm{~mm}$ A. 5235

Pileus $7-8 \mathrm{~cm}$. cinnamon brown, unbonate; stipe $7 \times 1 \mathrm{~cm}$ equal

A. 5408

Pileus $10 \mathrm{~cm}$ mars brown to honey color; stire $6 \mathrm{~cm} x$
11-5 mm taperine downward

Marein aliendiculate; " pileus $7 \mathrm{~mm}$, antimony yellow

pulem $2.5 \mathrm{~cm}$ Proutis bionen A goerger $9 / 9 / 36$

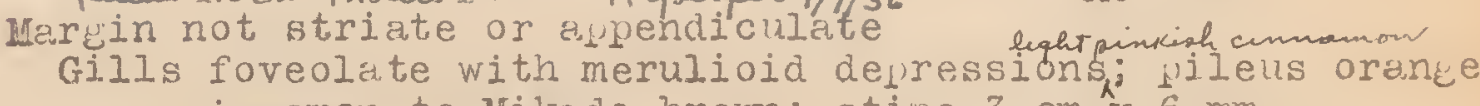

cimmamon to Mikado brown; stipe $3 \mathrm{~cm} \times 6 \mathrm{~mm}$

Gills not as above

A. 5134

Gills white

Pileus $1.5 \mathrm{~cm}$ ochraceous tawny to warm buff; stive $3 \mathrm{~cm} \times 3 \mathrm{~mm}$, warm buff

A. 5135

Dileus $2.5 \mathrm{~cm}$ white?; stipe $3 \mathrm{~cm} \times 10 \mathrm{~mm}$ white

A. 5217

Gills walnut brownto carob brom; wileus $8.5 \mathrm{~cm}$ sayal brown to snuff brown; stipe $2.5 \times 2-1.5 \mathrm{~cm}$

Gills free aill concolorous A. 5285

spores Erreen

Chlorophyllum molybdites

Spores whj.te ppleuswith claycolon des shading punknahbuff 5.7326

Pileus viscid \{ pilens all whte Lepiota (Limacella) auricola

Pileus tranulose or verrucose withswollen vestofles $1-2 \mathrm{~cm}$

fepiota (FuLejiota) aspratelia

Pileus pruinose, fibrillose or pulverulent, rarely elabrous

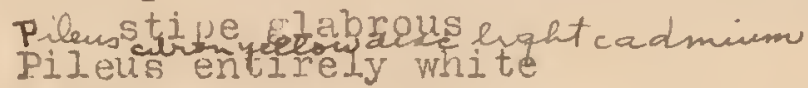

spores ovoid, 5 u

L.9090 Dulce 7109

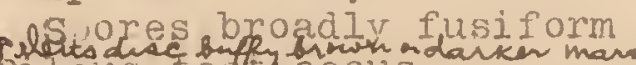

Pleus testiceous

pileus $1 \mathrm{~cm}$; stille and annuIus testaceous

Prpileus 4 mistipe and annulus whi.te

I. lactea

I. 7327 imensis

I. subyranuilosa $\} 7704$

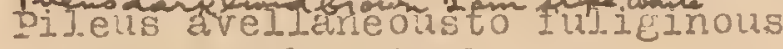

pileus umbonate $1-2 \mathrm{~cm}$

pileus not umbonate, $3 \mathrm{~cm}$

2. 7265

I. rimosa

I. Broadwayi

pileus siumamulose; annulus fixed or evanescent; scales a. ressed

Paleus carnelian red ti maceosis rufores $2.5 \mathrm{~cm}$ atipe whits 2.7262 


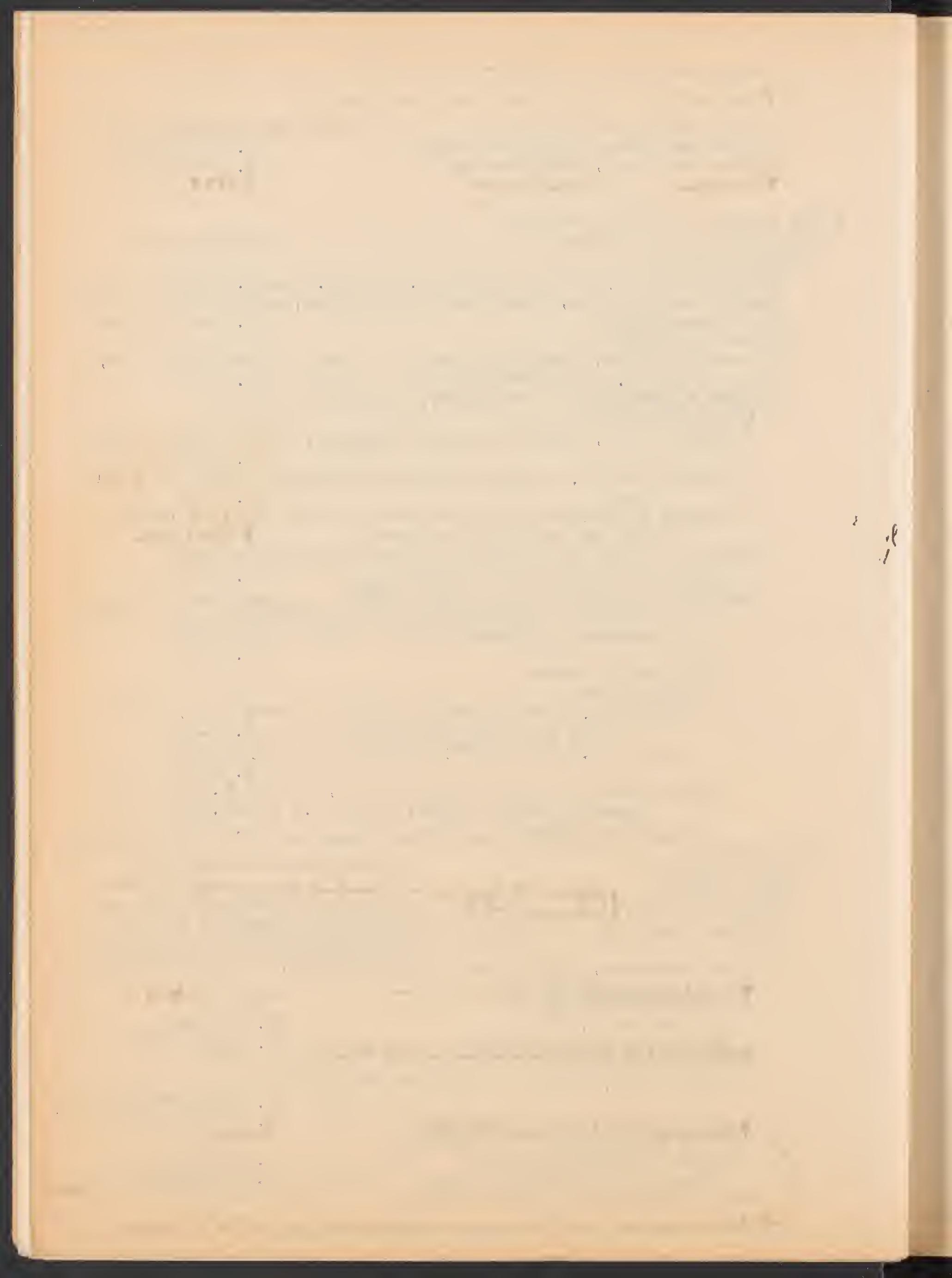


Pileus becomine reddish on wourding $10 \mathrm{~cm}$; expizlous cespitose Lepiota(EuLeriota) jamaicensis

Pileus not chaneine on woundine

scales some shade of red, fulvous or isabelline; pileus 6-7 cm;stipe $7 \mathrm{~cm}$, abruptibulbous L.abruptibulba $\underset{7278}{7275}$

scales avellaneous; pileus $0.7-2.5 \mathrm{~cm}$

Pileus pere than $1 \mathrm{~cm}$; stipe white I. suberisea

Pileus less than $1 \mathrm{~cm}$; stipe purplish fuliginous

I. subcristata

Scales brown to fuliginous; lileus $2.5-4 \mathrm{~cm}$, white not

c chancine on dryine I. tepeitensis 7692

pileus conspicuously long striate, Thin, scuamulose

Pileus 5-10 cm yellow, cespitose, stipe bulbous; spores $8-1$ $8-11 \times 5-7$

I. cretaceus

I. cepastipes

Pileus $1-5 \mathrm{~cm}$

Pileus white wi th yellow center

Dileus white with dark scales; $2-5-5$

stipe $4 \mathrm{~cm}$, subecual Losubclypeolaria

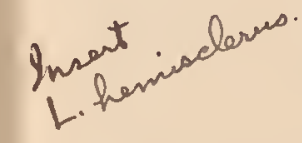

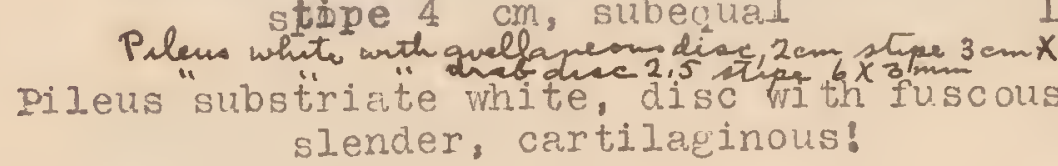

I. flavodisca 7305 $\mathrm{cm}$; stores $6-7 \mathrm{u}$;

$x 1 \mathrm{~mm} 5.3233$

I? 5505

Volva and yeil present

Pileus $5 \mathrm{~cm}$, convex; stipe $4.5 \mathrm{~cm} \times 4 \mathrm{~mm}$ without bulb

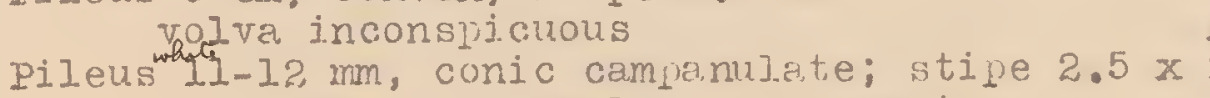

A. mexicana.

flatened above, volva evanescent,

Paleus 2.5 cmernoex stipe $6 \mathrm{~cm} \times 2-3 \mathrm{~mm}$ with bulb flattened above volva unconsprenous a. Golfo Dulce 7673

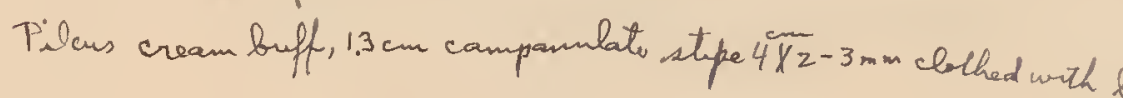
a. rubescens 

Spores pink

Volva and annulus wanting

stipe cartiliteinous

marcin of pileus incurved

Gills decurrent,

Iileus white

Pileus tan colored

Pilelis $1 \mathrm{cr} ;$ stipe $2 \mathrm{~cm}$

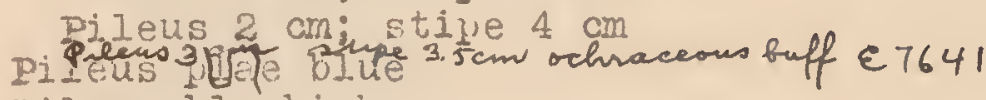

pileus blackish

Iscojija

3. 5592

R. cubensis

Th. Earlei

F. mexicane.

F. jamaicensis

Gills adnate or adnexeot

Leptonia

Eijixylous

Pileus $2 \mathrm{~cm}$; avelleneous; $\varepsilon i$ l. Is distant; stipe $3.5 x$ $2-3 \mathrm{~mm}$, murinous

I. a.trosu uamosa

Pileus 2.5. fawn color to avellaneous; gilla close

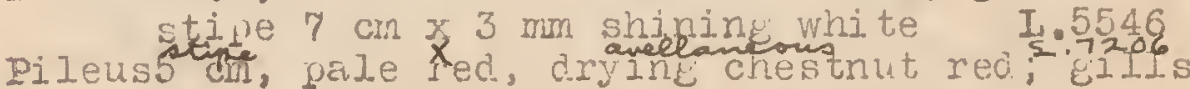
stipe $8-10 \times 3-5 \mathrm{~mm}$ concolorous with orance bese

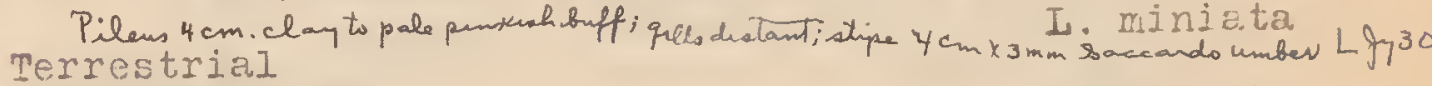

Pileus jale tan

Pj.leus livid purple

Pi.eus murinus or avelianeous

Stije 4-5 am, murinous

pileus avellaneous shadine to tilleu]. buff marein gills close; stine white, light brown when bruised

I. . . - , 5559\%

pileus murinous to margin; dil].s distant; stive

$$
\text { murinous L. murinax }
$$

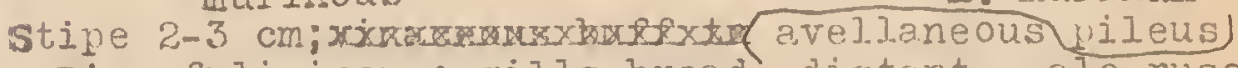

Disc fulieinous; gills broad, distant, fele russet stipe fumosus

I. cinchonensi.s

disc concolor; eills broad, distant wale ashy erey

Pilens pale punmens biff $4 \mathrm{~cm}$.

I. mexicana

L. 3730

Marcin of pileus straight; שills Bree or adnexed Nolanea wer

Pi].eus umbilicate

Pileus convex octue sad shagrg himate slups smoothwhte U.7678

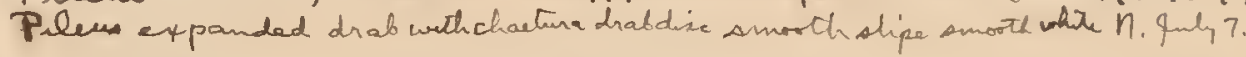

Stive fleshy

Gills decurrent, varyint to adnate

Pileus white, $1-2 \mathrm{~cm}$

C].to,ijus

Dileus Eray, $5-10 \mathrm{~cm}$

C. Jarlei

C. abortivus

Gills free

Pluteus

Pileus $1 \mathrm{~cm}$ or less broad

surface white covered with soft blackishhirs

Surface reddish wite, Elabrous

P. teihrostictu?

surface orance red.

P.a.lborubellus

Surface date brown

P. Iaetifrons

F. a.etra. Ius

Pileus $2-3 \mathrm{~cm}$, reaching $4 \mathrm{~cm}$ in P.compessipes

Pileus whitish with yej.J.oish disc

P. myceniformis 
Puleus umbelicate

Nolanea helicta

Pilens convers. palie fuacono

avellaneons unber to fuliginous struate conce n. cubsomsis drab with chaetura drab diac smooth not unbinat famaicensis

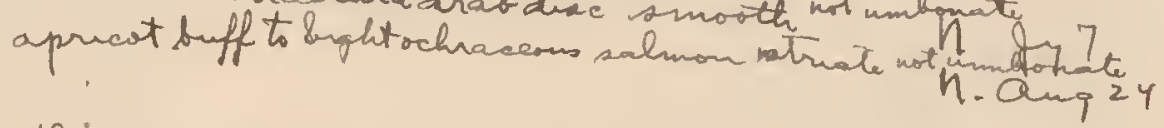

Pilens llat, hygrophanous.

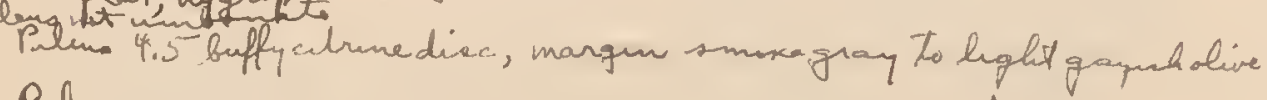
Pilens 2.5

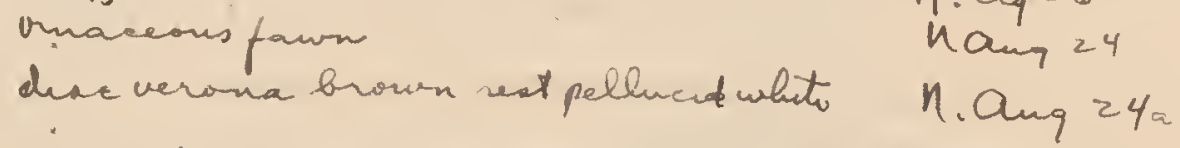

n. 0 q 26

Pilens unbonate, umbo saccardoumber, niangun bay al srown

n. $\operatorname{ang} i 1$

4 
Dilel.s rosy isabel.line, sliehtly darker on disc

Maryin striate; stire com ressed $30 \times 2-3 \mathrm{~mm}$

pluteus compressipes

Margin not stiate; stipe terete $50 \times 7-3.5 \mathrm{~mm}$

P. 5031

Pileus dark brown or fuliginous rarely varyine to olivaceous

Stipe brownish, $2 \mathrm{~mm}$ thick

stipe $7 \mathrm{~cm}$

Stipe $2 \mathrm{~cm}$

stife barium yellow

Stive white

Pileus dilabrous

surface striate

$\begin{array}{ll}\text { Stipe } 3 . \mathrm{cm} \times 2-3 \mathrm{~mm} & \text { P. multistriatus } \\ \text { Stipe } 8: \mathrm{cm} \times 4 \mathrm{~mm} & \text { P. } 5223 \\ \text { urface ruese, not striate } & \mathbb{P} \text {. jameicensis }\end{array}$

Surface ruese, not striate

stipe $1-3 \mathrm{~cm}$; pileus fuscous brownor olivaceous

stipe $3-4 \mathrm{~cm}$; wileus pale fuliginous to ditrk: chestnut P. Harrisili732.3

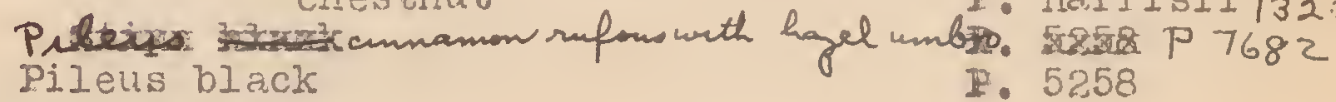

Pileus $4-5 \mathrm{~cm}$

stipe 3-4 min thick

Pileus glabrous, smooth

Pileus marein striate, stipe $10 \times 7 \mathrm{~mm}$ uray

P. 5030

Warein not striate, stipe $4-5 \times 3-4$, concolurous

Pileus 6-10 cm

P spinulosus

Surface densely floccose, uriformly wale yellow

Sunface minutely fibullose with pun to Tarlei

surface subgiabrous.

Tarein striate houm sitem

Warein not striate, stipe smooth $8-15 \div$ in $7-12 \mathrm{~mm}$

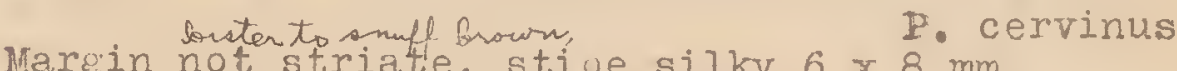

Marein not striate, stipe silky $6 \times 8 \mathrm{~mm}$

P. 5380

\section{Dileus velvety, reticulate \\ stipe terete, straw color $4 \times 5$ \\ stipe compressed pink 6 × $4 \mathrm{~mm}$ \\ Stipe $10 \mathrm{~mm}$ or more thick \\ litipe $5 \mathrm{~mm}$, mens vincid firielore!}

Volva wanting; annulus present ower

Volva 1resent; annulus wantiné

Pileus white or whitish, sometimes darker on dis
Terrestrial

Epixylous

Pileus $5 \mathrm{~cm}$ broad; stire $3-5 \mathrm{~mm}$

Pileus $6-10 \mathrm{~cm}$ broad; stipe $8-16 \mathrm{~mm}$

Dileus dark brown or fulieinous

Stipe $7 \mathrm{~mm}$ thick $\mathrm{ck}$.
P. reticulatus

P. 5029

P. rimosus 728

Chameota Broadway 1

Volvaria

V. Darlei

V. jameicensis

$V$. hombycina

$v$ cubens is

V. Bakeri 

Plens dark red drying reddesh brown gells narrow dryung redduch hrown

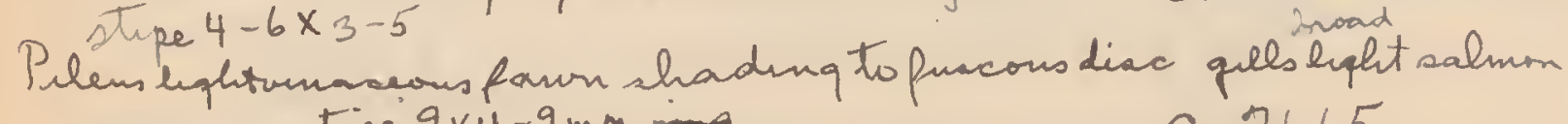
orange stipe $9 \times 4-9 \mathrm{~mm}$ ing $\quad$ C7665 
Spores brown

Volva and annulus absent

Pileus irretular, dimidiate or resuinate

Pilells 2-10 mm (reealas C. cuncifformis)

Pileus floccose mlverulent, $\begin{cases}\text { mare white } & \text { C.parvulus } \\ \text { pileus elabrous } & \text { c gylo }\end{cases}$

Pijeus 2-5 $\mathrm{mm}$ broad

Surface russet white or isabeliine

stipe inconspicuous, fueacious

stipe conspicuous, persistent

surface chrome yellow

Bileus $5-10 \mathrm{~mm}$

Dileus avellaneous or lizhter

pileus pele ochraceous

Pileus testaceous to latericious

C. Citri

C. eccentricus

C. Dussii

C. 5554

C. Psychotriae

C. bicolor

pileus $1-6 \mathrm{~cm}$, sometimes smaller in $C$. cuneiformis and $C$. subcuneiformis

Pilelis white or whjtish

pileus striate on the marcin

Marein very thin, pellucid, becomjng dark on drying

Margin thicker

pileus not stjate on the marcin

Dileus $1 \mathrm{~cm}$ broad

C. cinchonensis

C. sulce.tus

Di.le $2-3 \mathrm{~cm}$

c musa,ecola

Surface with small, brown pyramidal fibrils

surface glabrous

c. 4812,7318

C. fumosifokius

Pileus $6 \mathrm{~cm}$, viscid, glabrous, edees of vills flagcdse

Pileus yellowish, isabelline, ochraceous or melleous

Pileus about $1 \mathrm{~cm}$ broad

surface elabrous or nearly so

pileus sessile

Pileus attached by dark breddishbrown

C. sidbouneiformis

surface squamulose

stivie

C.substipitetus

C.cacaorhy l.u.

Pileus $3 \mathrm{~cm}$ broad, decorated with minute conic eleve.tions

Pileus $5 \times 3.5 \mathrm{~cm}$, ochraceous buff, minutely tomentose; Eills amber brown

c. 5547

Pileus 6 x 4 cm, fibrjilose scaly, disc snuff brown shadine to cream buff, sessile, eills decurrent

c. 5129

Pileus ochraceous red, pulverulent, deenly lacerate

C. Ieceretus

Pileus rufous, elabrous, not lacerate, sometimes Jobed

C. oyrrhus

Pileus buckthorn brown, blackening, Eills cinnamon buff, thick, crisped, interveined, decurrent c. 5049

Pileus clay color $2.5 \mathrm{~cm}$, eills cream buff, sessile

Dileus pale brown or watery brown

C. 4981

pileus $8-12 \mathrm{~mm}$ lamel. lae serrulate

C. cuneiformis

Pileus 1-2.5 cm; Uj.lls rot serrulate

pileus clove brown, faintly stricte,

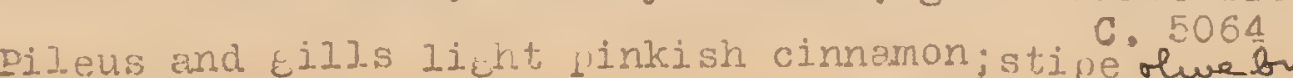

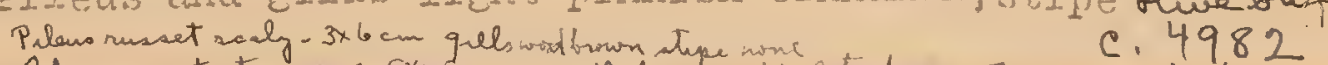

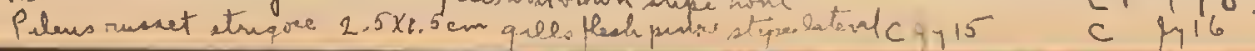




$$
\text { .4. ". . . - . }
$$


Tileus centrally stivitate

Stipe cartilarinous

Gjices ppánate lyzine

qullonstap. Hilis decurrent.

Pileus dark brown, mIverulent; stipe 4 unm thick

Paxillus 6137

Tubaria

T. coniophora

Gills adnate or adnexed.

Margin strajuht and apressed Galera

stipe $7-20-237$ lone

pileus $3 \mathrm{~mm}$, tawny to russet; stipe $23 \mathrm{~mm}$

Pileus $5 \mathrm{~mm}$

G. 5106

Epixylous

Terrestrial on elay banks

G. Martiana

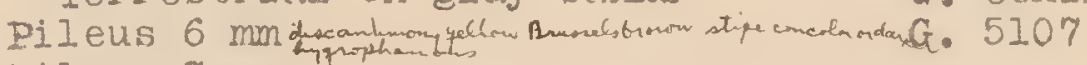

Dileus $7 \mathrm{~mm}$

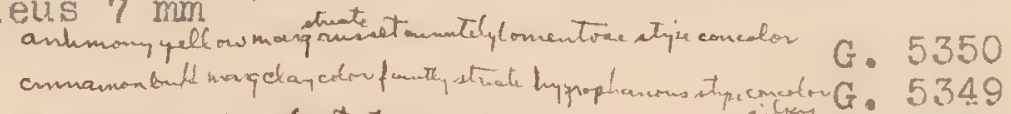

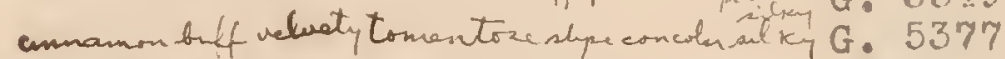

Pileus $10 \mathrm{~m}$; ilis distant G.distantifolia

Pileus 12 m; EilJs close; mumrybrown with echinate

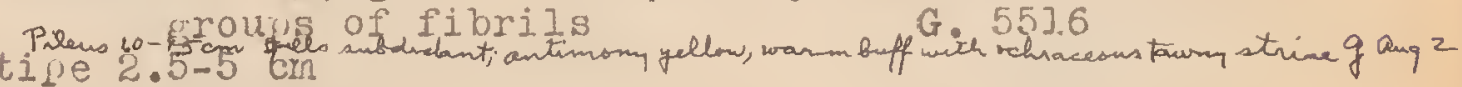

pileus cylindric G. macromastes

Pileus subconic or campanulate G. Hypni,73/4

Pileus becomine dewressed with maryin reflexed

Stipe $8-12 \mathrm{~cm}$

G. reflexa

pileus $1-2 \mathrm{~cm}$, marein striatulate

Surface smooth

Surface subsuluamulose

G. tenera

G. flocculenta, Fr.?

pileus 2-6 cm

Marein striatulate

Margin not stiatulate

G. crisla

G. mexicana

Maruin incurved (see also N. arenicola) Naucoria

pileus white or whitish

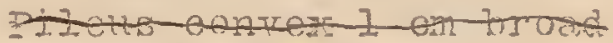

surface elabrous

Pileus convex, $1 \mathrm{~cm}$ broad

Pileus umbonate, $2.5 \mathrm{~cm}$ broad

surface papulose; pileus $2.5-5 \mathrm{~cm}$ broad

H. the reztentis

N. tepeitensis

N. ja.layensis

N. wepularia

1. subolivacea

Pileus dull olivaceous, $1 \mathrm{~cm}$ broad $\mathbf{B}$, sub
pileus testaceous with bej umbo, 7 mo broad.

pileus vinosus, $6-8 \mathrm{~mm}$

N. pellucida

N. ojnoides

Pileus ochraceous, isabelline or fulvous, sometimes differently colored on disc

pileus $0.7-1.5 \mathrm{~cm}$ broad

Surface Elakrous, fulvous

Surface innate fibrillose, isabeline

Gill. wele ochraceous

N. cyathicola,

Gills bay-fulvous at maturity $N$. corticole.

surface hisuid-s tuamulose, fulvous

I. meyicana

n. 5010 


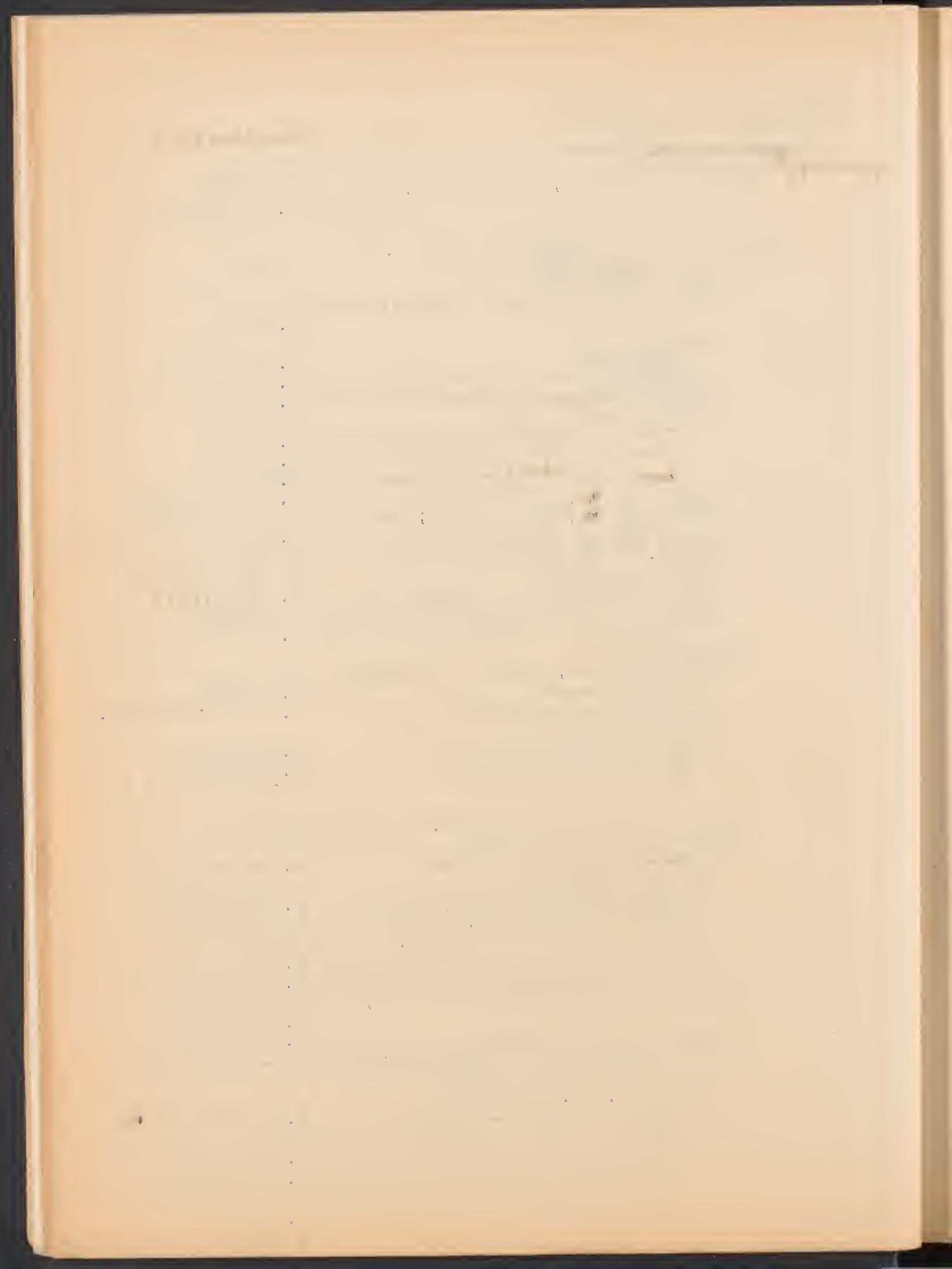


Pileus $2-5 \mathrm{~cm}$ broad

Stjpe $1.5 \mathrm{~cm}$; pileus umbonate ochraceous tawny with cinnamon brown disc, marejin not, stiate, but

atrendicil.ate N. 51.64

stipe $2-2.5 \mathrm{~cm}$, sometimes longer in $\mathrm{N}$. subpectinata

PiJeus ochraceous with bubfulvous disc, si ichtly depressed

Pileus cinnamon brom, convex N. 5083 viscid

Pileus isabeline with estaceous disc, umbonate

Pileus fulvous, dejressed, striate N!. spinulifer

I. . subpectinata

stipe $3-4 \mathrm{~cm}$ maid

viscid\{ril eus bay to buff, stipe $4-5 \mathrm{~mm}$ diam,

Pidens cinnomon buff, stupe $1.5 \mathrm{~mm}$ dian n.quhy 14 NT. 51.67

DiJ.eus alutaceous, stife $2 \mathrm{~mm}$ diam

N. Barlisi

Pileus ochraceous orance, stipe 2 mun diam

Pilens warm buff stype $2-3 \mathrm{~mm} \quad n, \mathrm{f}_{7} 28$
Stipe $4-8 \mathrm{~cm}$

Gill.s close

Gills distant, fus@ us
PiJeus some shade of lanbrinous or brown, valabrous, $1-2 \mathrm{~cm}$

N. 501.1.

N. semiorbiculeri 1237 stive $1-2 \mathrm{~mm}$

Pileus umbonate

Margin striate

Marein not, stricte

N. montana

N. 5165

Pileus donvex, marein not striate $N$. Sacchari

PiJeus depressed; maruin not striate N. 5159

stipe $3 \mathrm{~mm}$ thick, pileus conves, maruin not s.triate

N. underwoodi

pileus auburn with conical, bay umbo, marinstriate, $4 \mathrm{~cm}$

N. 5009

Gill.s free

Pluteolus

Pileus $2 \mathrm{~cm}$, umbonate fibrillose, florssels hrown, stive

$$
4 \mathrm{~cm} \times 2.5 \mathrm{~mm} \text { P. } 51.11
$$

Dileus 3-4 cm;not umbonate, Elabrous, isabelline, stipe

Gills autolyzing

$$
10 \mathrm{~cm} \times 5 \mathrm{rm}
$$

Plieus flavo-melleous or sulfurl yeljow. stipe elabrous Stipe viluose

Pileus avellaneous, hrowish on the disc Pileus $12 \mathrm{~mm}$; stipe $6 \mathrm{~cm}$

Dileus $2 \mathrm{~cm}$; stipe $2-4 \mathrm{~cm}$

P. jamaicensis

Bolbitius

tawmy on disc

B. jalapensis

B. villipes

Pileus japan rose to pleshcolou mangin puleus $1.5-2 \mathrm{~cm}$ stype $3-4 \mathrm{~cm}$ B. 3306

stipe fleshy to yroody

Gills adnate to short decurrent;

Terrestrial.

stipe 2-4 $\mathrm{mm}$ thick

Pileus dry

Pileus viscid

Stile 6-8 min diam $2-2.5 \mathrm{~cm}$ lone: dry

stipe $10 \mathrm{~mm}$ diem, $8 \mathrm{~cm}$ Ione, dry
Flammula

F.hy phojomoides

F. carbonarius

F.jelapensis 


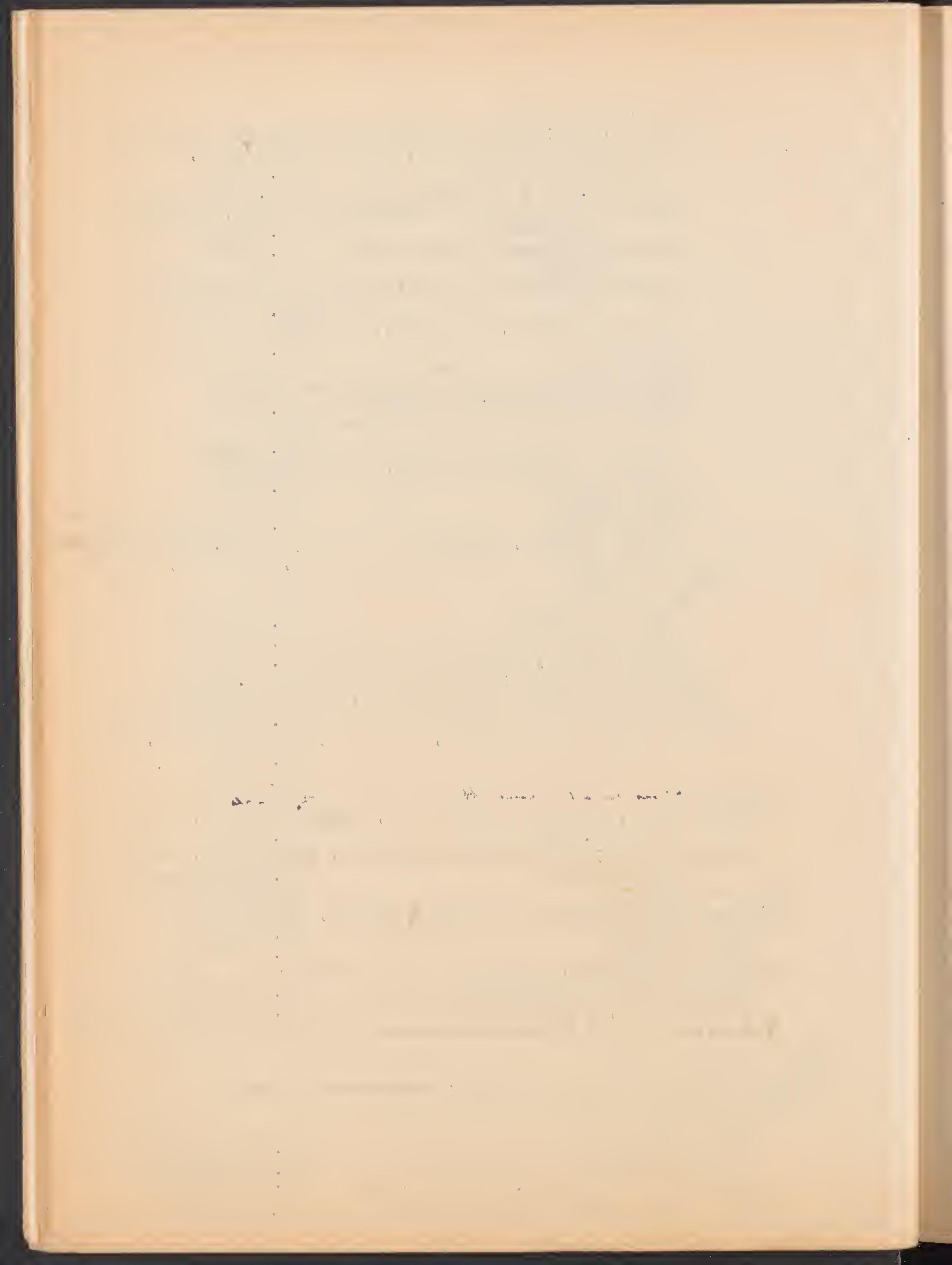


Fipixy]-ous

Pileus Elabrous

Dileus amber brown to antique brawn, slichtly umbonate stipe $18 \times 6 \mathrm{~mm} \quad$ Flammula, 5172

Pileus pel.low-olive; stipe $1.5 \mathrm{~cm}$ F. olivaceus

pileus jellowish orance to Ereenjsh; stipe $4-7 \mathrm{~cm}$

F. aureoviridis

Pileus melleous, ochraceous or luteous, stipe $3-5 \mathrm{~cm}$

F. flavidellus

Pileus ferruginous to fulvous; stipe $4-6 \mathrm{~cm}$

Pileus ferrueinous vinous; stipe $2.5-3 \mathrm{~cm}$

F. chrysotrichoides

pileus red tinged with vinous; stive $3-4 \mathrm{~cm}$

F. hèriolicens

F. vinicolor

Pileus fibrijlose to sulumulose, sometimes becomine more or less glabrousin ace

Pileus éldenyel.low

stile $1 \mathrm{~cm}$, eills adnexed

stipe $2-5 \mathrm{~cm}$

Tawify maculate, musta'd yellow $u_{1}$ 'to '2 cm; stipe

$3 \mathrm{~cm}$, slender

F. 4642.7233

Not maculate $2.5-4 \mathrm{~cm}$; stipe $2.5 \mathrm{~cm} \times 8 \mathrm{~mm}$

Not maculate, rus'k to antimony $13^{\prime \prime}$. chrysotrichus

Iot maculate, russet to antimony yellow; stipe

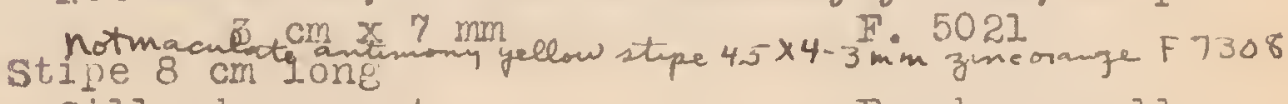

Gills decurrent F. chrysopelia

Gills adnate

F. aureobrunnea

Pileus pale jellow to ferruginous oranee

pileus about $1 \mathrm{~cm}$, umbilicate, umbonate, stipe xantinin orange to ainc'órange

pileus $2-4 \mathrm{~cm}$, rarely reachine $6 \mathrm{~cm}$

Pileus umbilicate or depresseo

Stipe $2-2.5 \mathrm{~cm}$

pilelis contex to ext anded

Stipe $2-4 \mathrm{~cm}$

pileus amber brown $13 k$

Pileus auburn $11 \mathrm{~m}$

Pileus raw sienna 17

Pileus ochraceous 17'

Taste mij.d

Taste bitter

Margin striate

Margin not, striate

pileus ferrueinous $9^{\prime} i$

Pileus ferrueinous orane

Pilelus chestnut $9 \mathrm{~m}$

F. 5520

stipe $4-7 \mathrm{~cm}$ lone

Dileas ochraceous 17 !

Pileus pa.le ferruginous

Pileus $6-10 \mathrm{~cm}$ broad

Pileus deeply depressed

Dileus not depressed

F. hispidus, 7650

7. $\operatorname{colmicola}$

F. 5169

F. 5179

F. 5181

F.pholio toides

F. parvujus

F. hispidel.Jus

F. bryouhijus

F. subpenetrans

F. 5176

F. Nashii

F. Farlei

Gills decurrent

stipe $4-6 \times 5-8$

F. defressus

F. tenfuis 


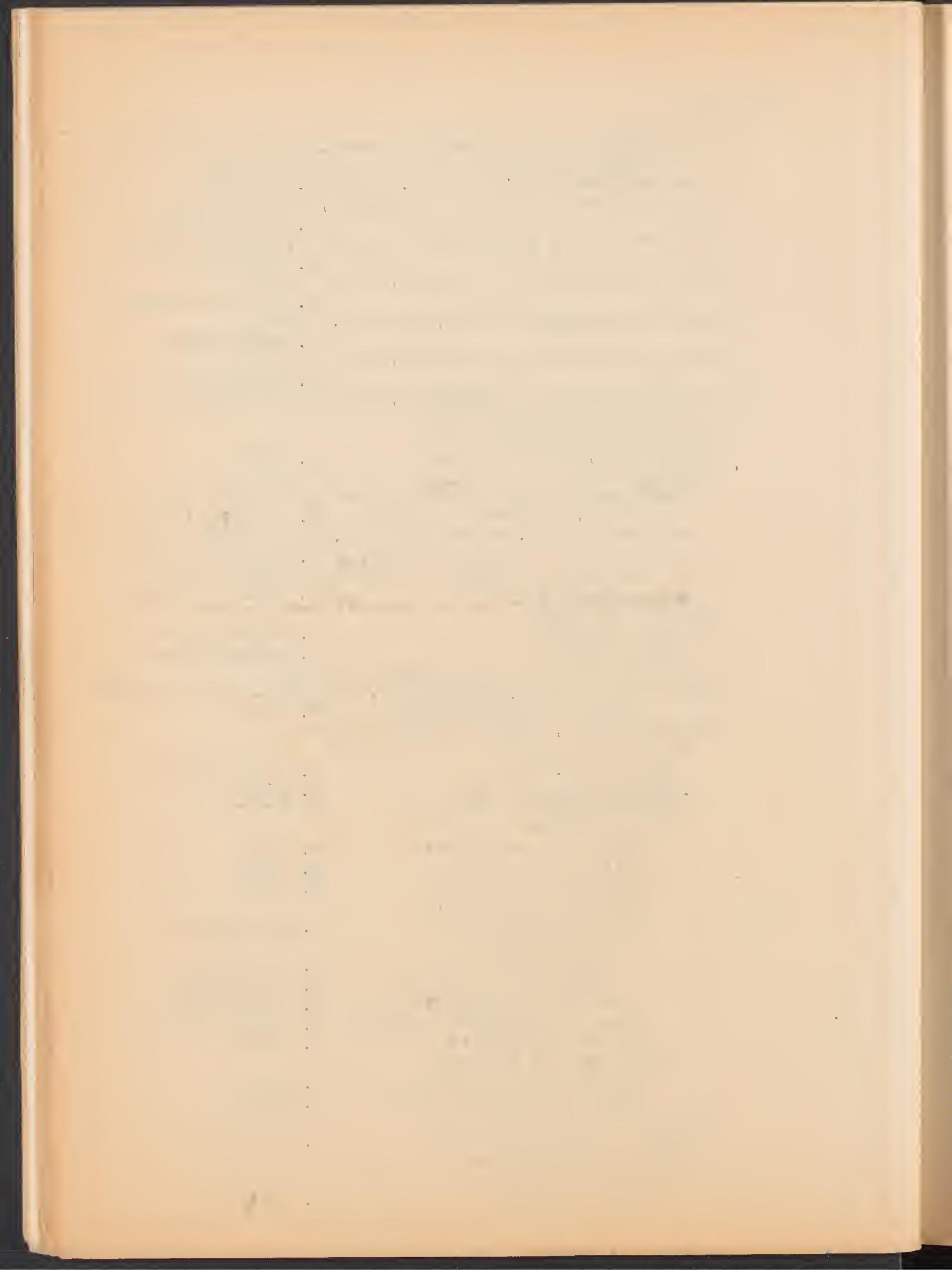



Stipe $2.5 \mathrm{~cm} \times 5 \mathrm{~mm}$
Gilis adnate
Pileus fulvous $13^{\prime} i$
Pileus latericious $5^{\prime} x$
Flammula 5177
F. areolatus
F. penetrans
F. Iateritia.

Gills sinuste or adnexed, terrestrial

pileus Elabrous and viscid

pileus white or nale isabelline

Stipe $2-4 \mathrm{~mm}$ diam

stipe 7-10 min diam

pileus pale testaceous, scarcely

Pileus incarnate isabeliline, $2-2.5 \mathrm{~cm}$

Hebeloma

Pileus chestnut brown

H. broadwayi

H. cinchonense

Pijeus mummy brown to dresden brown with white marein 8-1.0

Tileus sabelline shading to colomal buff $3 \mathrm{~cm} H .6128$

Volva absent; annulus jresent

stipe Elabrous, fibrillose

Pileus laree $3-12 \mathrm{~cm}$; tawny to cinnamon brown

stipe pale yellow, $3-6$ m $x 3-10 \mathrm{~mm}$

stipe soon blackening, $10 \mathrm{~cm} \times 1-3 \mathrm{~cm}$

Pholiota

H. subtestaceum

diam

H. subincarnatum

H. cubense

Pileus much smaller, up to $4 \mathrm{~cm}$.
P. cubensis
P. Brittoniae
P. 4647
P. 5378

Dileus $3 \mathrm{~mm}$; pale avellaneous; stive $5 \mathrm{~cm} \times 4 \mathrm{~mm}$, white

Pileus $5 \mathrm{~mm}$ to $4 \mathrm{~cm}$

Pileus white or isabeline; stipe concolorous or slishtyy Iighter

Pileus $0: 5-2 \mathrm{~cm}$; stipe $2 \times 1-2 \mathrm{~mm}$ P. bryorhila

pileus $1-3 \mathrm{~cm}$; stipe $4-7 \mathrm{~cm} \times 2-3 \mathrm{~mm}$ P. Broardwayi

pileus pale fuscous to tan, $1-4 \mathrm{~cm}$; stipe white, $4-6 \mathrm{~cm} x$ $3-6 \mathrm{~mm}$

P. Musae.

Pileus ochroleucous to ochraceous, $1.5 \mathrm{~cm}$; stipe $1.5 \mathrm{~cm} x$ $1 \mathrm{~mm}$

P. cinchonensis

Pileus cinnamon buff to ochraceous orane, $05-2 \mathrm{~cm}$; stive $2.5-5 \times 2-4 \mathrm{~mm}$ P. unicolor

Pj.leus reddish brown, $0.8-1.5 \mathrm{~cm}$; stive $2-3 \mathrm{~cm}$

P. martimicensis

Pileus vinaceous cinnamon, $2.5 \mathrm{~cm}$; stipe $3.5 \mathrm{~cm} \times 3-5 \mathrm{~mm}$

insent tuly 13,14

stipe squarrose scaly
P. 5372

Hypodendron

H. 5253
Volva present; annulus absent

Volva present; annulus present
Isocel.Iina quly 28

Rozites 


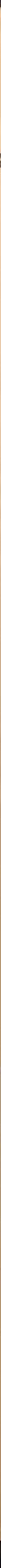


Spores purple brown

Pileus lateral or excentric

Pileus centrally stipitate

$=-\cdots 7316$

stipe cartilatinous, no annulus present

Lamellae decurrent

Lamellae adnate or adnexed

Margin of pileus straisht

pileus white, umbonate

pileus bromish olive,

$6 \mathrm{~cm} \times 4 \mathrm{~mm}$ white

(1300m)

Deconica fy ang 2

Psathyra

P.4731

shading to chamois $3.5-4 \mathrm{~cm}$, wstipe

conic, maregin striate) (40m) P.7276

pileus huff or chajky white to fusous mareins, expanded, margin lacerate, broad umbo (1300)

$\mathbf{P . 4 7 2 3}$

pileus buff shading to whjte, expanded, marein substriate broad umbo, stipe white

pileus honey yellow to olive lake margin isabelline, cate, stipe wax yel.low

$(2250)$ of. Po.preadotanes

P. 5593

umbili-

pileus avellaneous to buffy brown, expended to hemispheric lowland except P. 5105

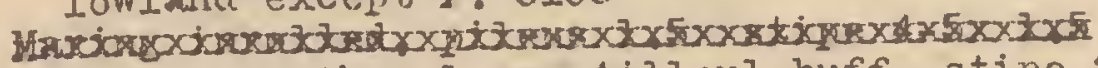

pileus less than $1 \mathrm{~cm}$, tilleul buff, stipe $2 \mathrm{~cm} \times \mathrm{lm}$ concolor, marein inrolled

P. 7278

pileus $1-2 \mathrm{~cm}$, stipe whj.te

Marejin inrolied, pileus $1.5 \mathrm{~cm}$ avellaneous stipe $4-5 x$ $1,5 \mathrm{~mm}$

Margin striate, pjileus $1-1.5$ cmwood brown to buffy brown, stipe $2 \times 0.5 \mathrm{~mm}$ umbonate

P.7292, 7638

Margin unknown, pileus $2 \mathrm{~cm}$, wood brown, stipe $3 \mathrm{~cm} x$ $2 \mathrm{~mm}(2.00)$

P.5105

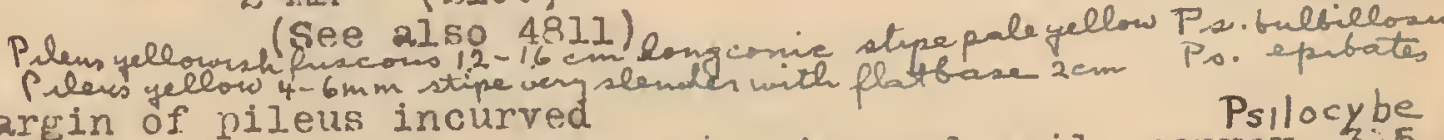

pileus viscid under scuamose universal veil, convex, $35 \mathrm{~cm}$ russet shadinf to antimony yellow; stipe russet, shagey below

Pileus not viscid, no evidence of universal veil, stipe silky smooth

Gills adnexed, pjileus campanulate, $2 \times 1.2 \mathrm{~cm}$, shinine white, margin smooth, stipe white $8 \mathrm{~cm} \times 3-4 \mathrm{~mm} .5529$

Gills sinuate adnate, pileus expanded stipe concolor or nearly so

pileus gibhous zinc orane to gale pinkish buff velrety P. 4733

Dileus umbilicate, honey yellow to olvefota shadjne to old gold

P. 5102

Gills adnate

Stipe concolor or nearly so, marein smooth

pileus $0.7 \mathrm{~cm}$ expanded tilleul buff P.7278

Pileus up to $4 \mathrm{~cm}$, campanulate disc merulioid, ochraceous orange to ochraceous buff, subtomentose

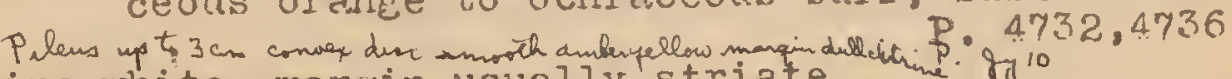

Stipe white, marein usuelly striate me g 10

pileus conic, $4 \mathrm{~cm}$ chestnut brown to avellaneous marejin strjate; stj.e $8 \times 2.5-3 \mathrm{~mm} \quad$ P. 5082

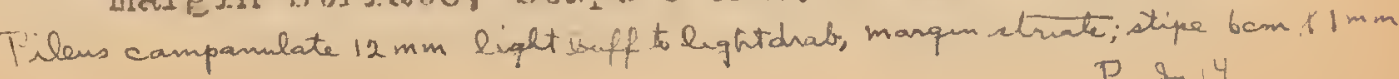



pileus conic exyanded $2 \mathrm{~cm}$ raw umber, marein striute stipie $2.5 \times 2$

Psilocybe 4.986

Pileus hemispheric, $3 \mathrm{~cm}$ honey yel.low shadine cream buff to cartridge buff, marein lacerate, striate stipe $4 \mathrm{~cm} \times 3 \mathrm{~mm}$

Ps. 4984

Pileus exiranded

Pileus tomentose, marein lacerate stive more then $5 \mathrm{~cm} \times 4 \mathrm{~mm}$

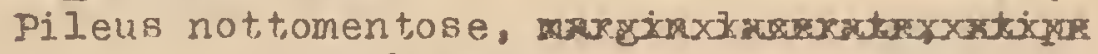
hyi rophanous

Disc white marein fuscous, margin lacerate, stive $2 \times 3 \mathrm{~mm}$ Ps. 4723

Disc buff, martin white, substriate Ps 5593

Gi1.1. free gray

annulus absent yom
Peleus dark frown slye $3 \times 3$

$P$ lem lown $2 \mathrm{~cm}$-Pi.losace 4815

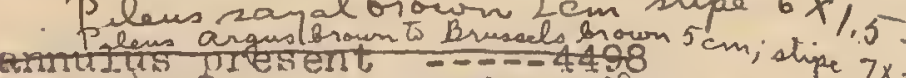

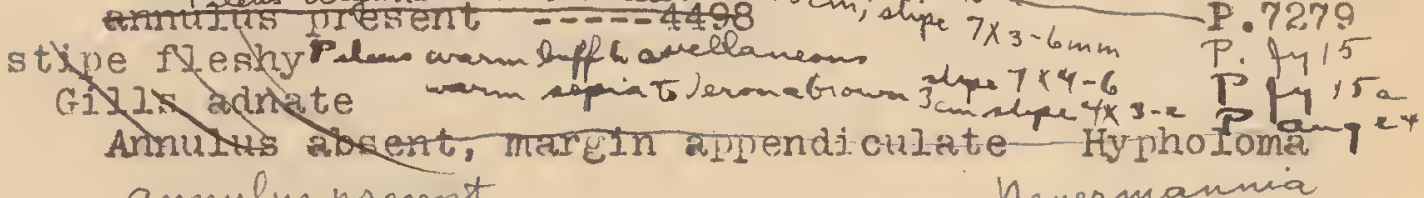

ammelus prosent

stipefleshy
quelsfree present
nnuius present
volva absent
pileus more

1.5409

thick

pileus under 7 s

Sting thick, $5-10 \mathrm{~mm}$

pileus Fitruscan red or darker

pileus ochraceous $(15-17)$

pileusochraceous buff disc warm buff stipe warm buff to white, tapering downward. Plemannomy yelownthem annulus broad, thick, single cte Ps6957

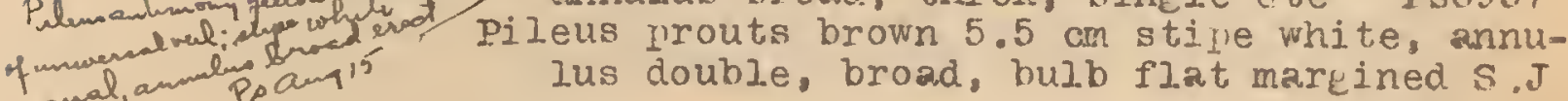
singlé

Pileus buckthom brown or liehter, viscid, Ps. 5376

stipe crenadine pink or liehter, annulus

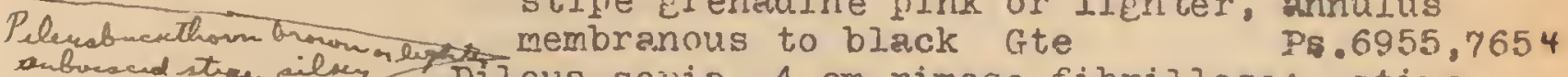

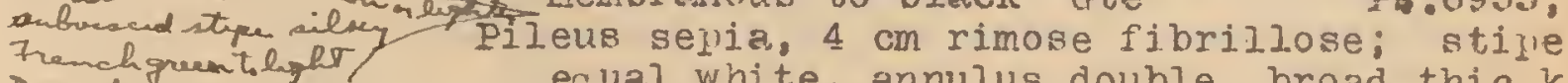
sremchgrumt.hget, equal white, ennulus double, broad thic $k$ S.J. Ps.5027

Pileus brown $6.5 \mathrm{~cm}$, tomentose scaly, stipe white tanerine upward, annulus thick, spreading

Ps. 48j.6, 4498

Pileus umber $6.5 \mathrm{~cm}$ sumamose, stipe white taperine upward, annulus evanescent Antilles

Ps. jejunus Fr.

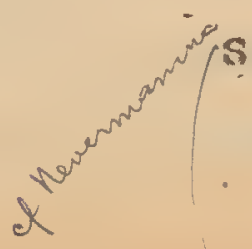
stipe slender $1-4 \mathrm{~mm}$ more or less cartilatinous low elevations (n.t.)margin striate Dileus wood brown, disc darker $4 \mathrm{~cm}$, stire white, annulus narrow to medium erect 4 cm below apex

Ps.7285 

Pileus pale ecru drab, disc orante vinaceous $4 \mathrm{~cm}$ stipe vinaceous cinnamon, annulus broad white, bulb $6 \mathrm{~mm}$ Pralliotsxx 7235

Pileus light cinnamon drab disc warm sepia $2 \mathrm{~cm}$ stive warm sepie, annulus narrow, erect, no bulb.

Ps. 7294

Werckle report, campestrist aruemsio

volva present

Clarkeinde, 5336 Annulus absent

Pilosace

pileus $10 \mathrm{~cm}$, stipe yellow rufous, $12-15 \times 6-8$ fuscous

P.tricholepis

Pileus $5 \mathrm{~cm}$, white, stipe white $5 \times 4-6$ bulbous

Gills adnate or adnexed

$$
\text { P. hololepis }
$$

Annulus absent, marein appendiculate Hypholoma

Pileus arricot yellow, eills adneted, stipe light buff shading to russet compressed H. 5407

pileus tawny shadingto buffy yellow $3 \mathrm{~cm}$ gills adnate stipe light buff shadingto tawny fibrillose

$$
\text { H.5243 }
$$

Annulus present Stroinaria

Yolva abseth

Volva present, becomine gelatinous,jileus viscid

$--.-5370,5160$

Spores cozl black

GiJ.Is not autolysint

Stipe cartilasjnous

Iamellae adnateur adnexed

Martin of pileus straicht

sunulus absent

Pileus and stipe becomine bleck; eills mante

pileus viliose, broad conic

pileus survoth, expondied

P8. 5132

Psathrreila

pileus ochraceous $(15-27)$. marein more or las ntrinte

Gills adnexeu. Nileus velvety, expinded camprulate. disc cinnimon, martins wood brown to drmb. stipe whitish $5 \times 2$

P6. 4997

Gills ainite

Pileus buffy brown, innate filurillose hemispheric naxin aljendiculate Ps. 4644

Pileus ochraceous buff, membranous camnanulate

Plans warm buff, hemepheric $P$ o. gy 13

pileus vinaceous fawn, campanulate

Annulus present

Maufin of pileus incurved

Pileus $1.6 \mathrm{~cm}$ buffy brown stipe drah

GiIIS free pale yeled stipe pale infous

stipe fleshy

Gills dinate no annulus present

Ricizx

Annulus and gelatinous volva present
Ps. 5085

PS. 7694

? 6135

Paneeolus

P. 4644

P. Phalenarmm ?6954

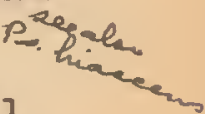

\section{8}




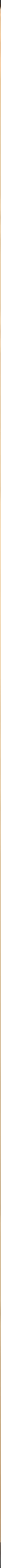


Gilzs autolysing

pileus all white, lacerate scuamose $6 \times 20 \mathrm{~cm}$, stipe $37 \times 20$ C. comatus?4953

pileus white with isabeline disc $3 \times 2$, stipe $11 \times 4$ C.7725 Pileus ochraceous $\left(15^{\prime}-19^{\prime \prime}\right)$

Pileus isabelline $4 \times 5$ stipe $15 \times 5$

Pileus chamois, $1 \times 1$, stipe $3 \times 2.5$

C7661.

Dileus dresden brown toavellaneous

C5059

pileus buckthorn brownto warm buff

C5060

pileus cinnamon brown to ochraceous tawny $3.5 \times 3$ C. 5109

see also C.armullaris Cephemenus, C. pheatilis, Cspoerstic/2 

inton

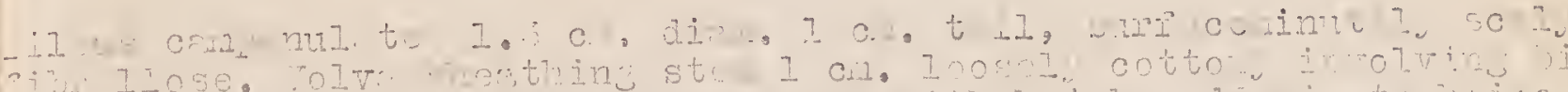

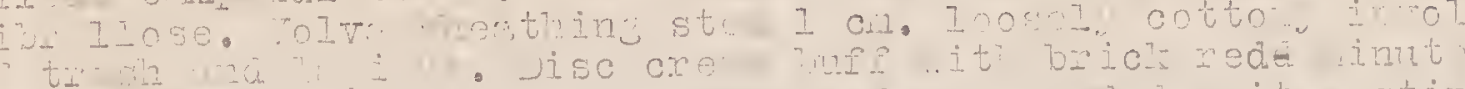

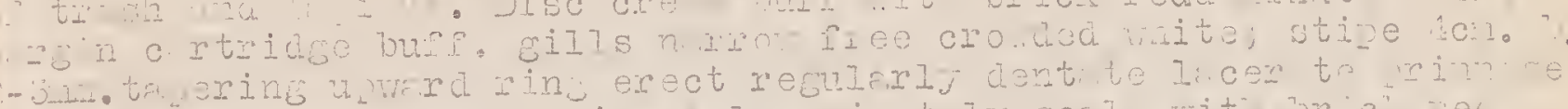

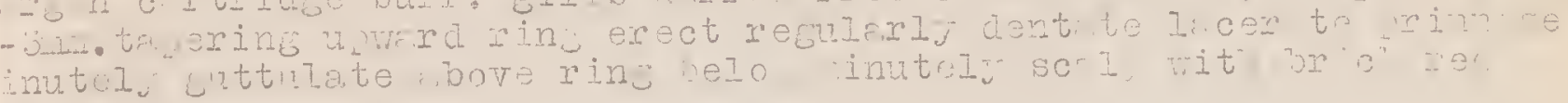
rioriI

C-stin $\quad 7-2 i-36$

$2+i \quad n \quad \ldots+i n=0$

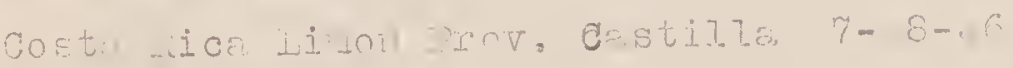


Amani ta

Pileus conic campanulate, white, $12 \mathrm{~mm}$ diam, cottony unirersal veil quite uniformly distributed over surface; mareins slightly inrolled, thin; gills close free, white, annulus thick erect; stipe $24 \times 2 \mathrm{~mm}$, taperingupward, b ulb flattened above, corered with a cottony universal reil which has a very cottony, evancescent free margin on outer edge of bulb.

on bank on roadside

Specimens examined

Costa Rica: San Jose, Rancho Redondo, 2200m Dodke \& Thomas 5001 


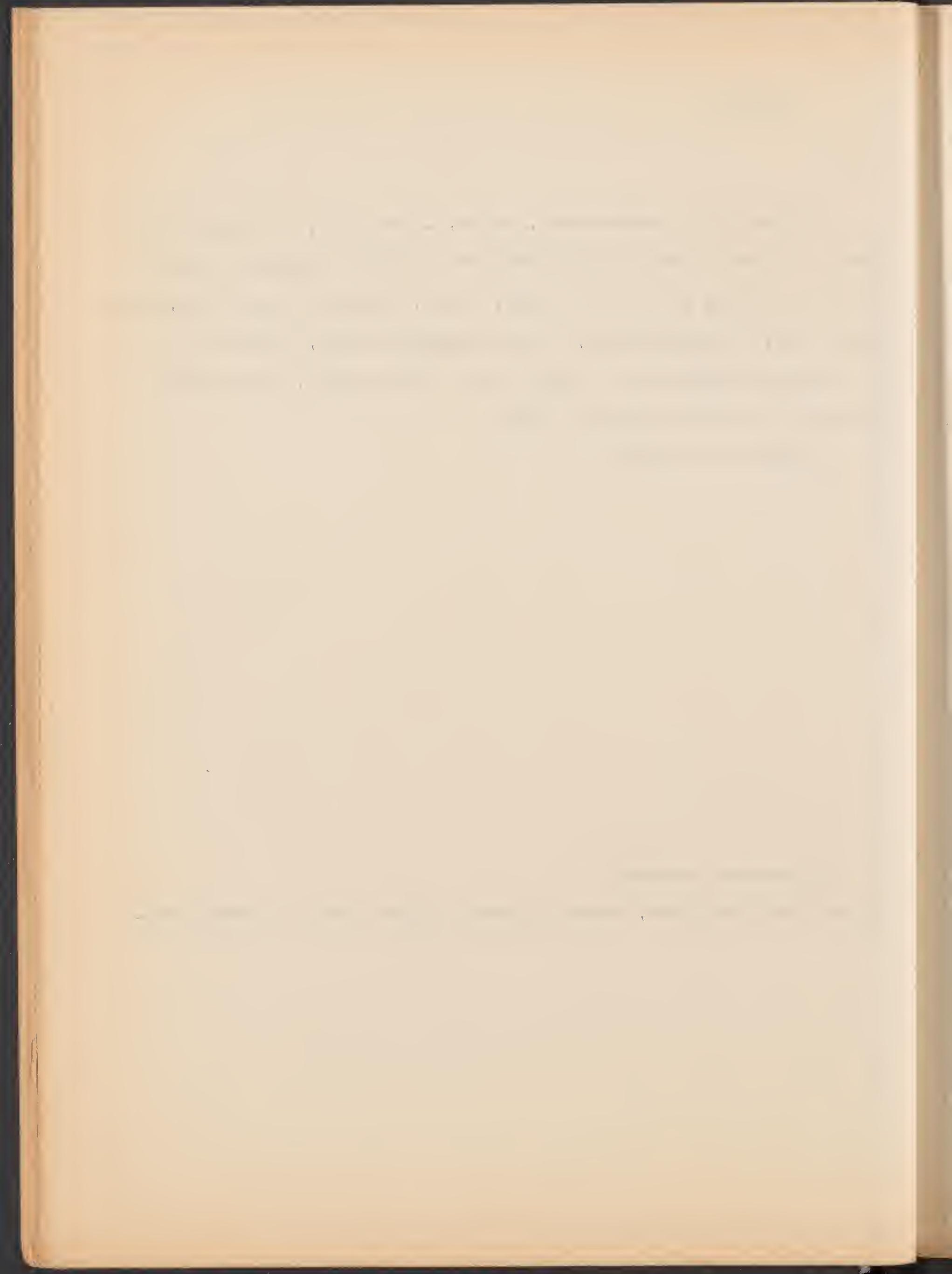


Amanita

Pileus conic, $11 \mathrm{~mm}$ diam, white, minutely fibrillose squamose with torn remains of universal reil; rolva membranous involvine particles of soil on its outer surface; gills free, narrow, crowded $\mathrm{cm}$ bulb $6 \mathrm{~mm}$

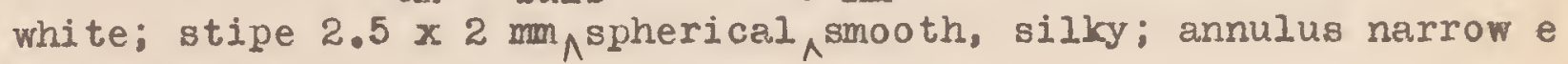
erect with a, a few partides of soil clinging to it.

Bank by roadside

Specimens examined;

Costa Rica: San Jose, Rancho Redondo, $2250 \mathrm{~m}$. Dodge \& Thom8, 5088 

Amanita

Pileus $2.5 \mathrm{~cm}$, convex, pure white, innate fibrillose, margin slicht Iy apprendiculate, smooth; gills free, ventricose, crowded white, ring inconspicuous, narrow, erect; stipe white, $6 \mathrm{~cm} \times 2-3 \mathrm{~mm}$, taperine upwardbulb flat with marrow mareinal volva

Costa Rica: Puntarenas, Osa, Bsterosandalo 5-20 m. Dodee 7673 


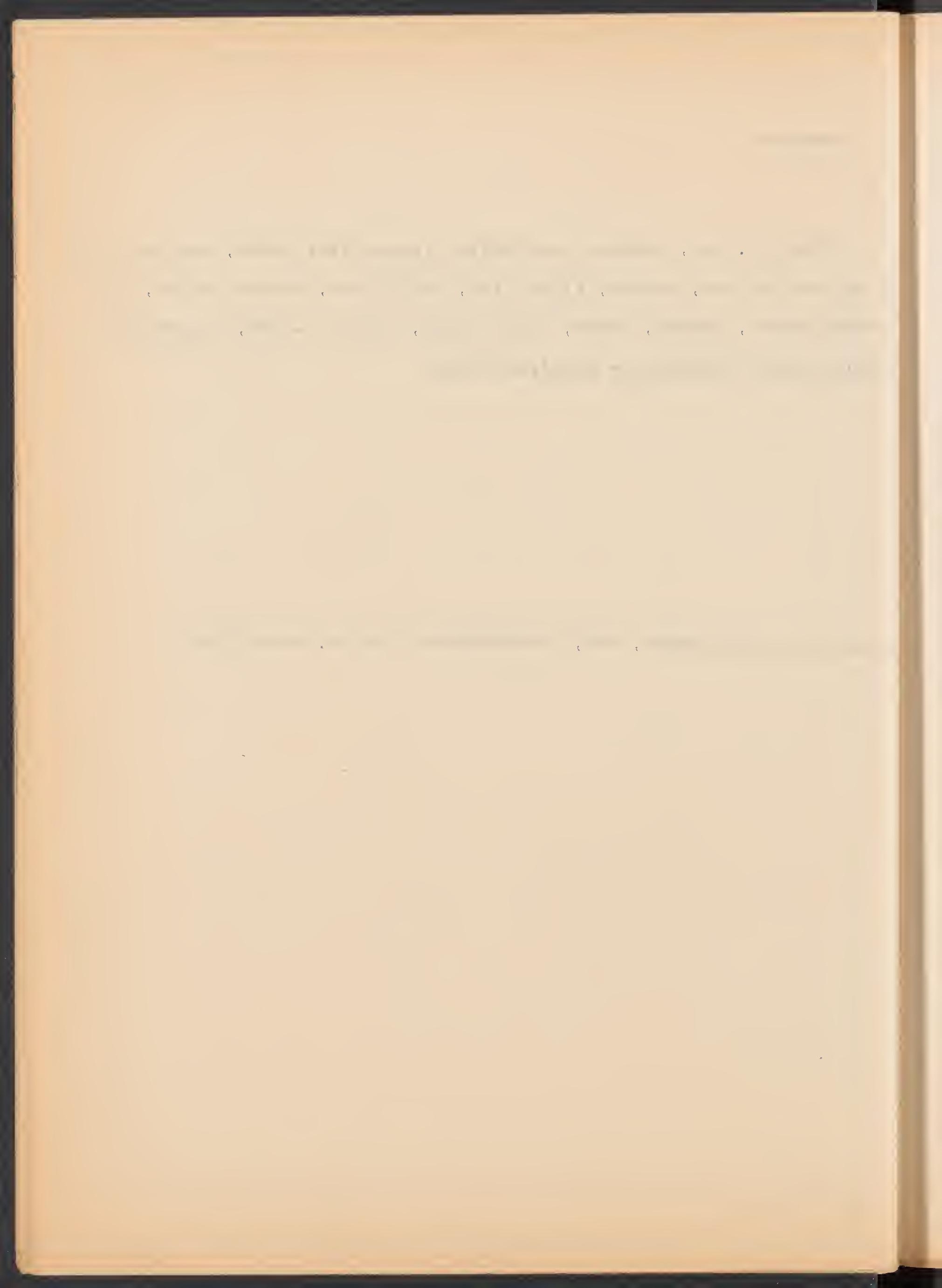


Armjlilaria

Pileus expanded depressed, $8.5 \mathrm{~cm}$, marein crenate in roxl.ed somewhat lacerate in age; surface fibrillose squamulose, sayal brown to snuff brown, flesh white, thin except on disc; gills walnut brown to carob brown, decurrent by line to ring which is narrow, thick, concolorous; stipe tajering cowmward concolor with pileus, fleshy fibrous $5.5 \mathrm{~cm} \times 2$ cmabove $1.5 \mathrm{~cm}$ below

In bare soil banks by roadside

Specimens examined

Costa Rica: San Jose, Rancho Redondo, $2250 \mathrm{~m}$ Dodge \& Thomas 5285 

Armillaria

Pileus expanded, moist, slightly viscid( remains of universal veil present, since soil clines to stipe from position ocupied by margin of pileus to end) russet, slightly lichter arround faintly striate marein; flesh thin on disc gills adnate with decurrent line to annulus, pinkish, medium width, close, regularly interveined stipe fleshy, $3 \mathrm{~cm} \times 8 \mathrm{~mm}$, innate fibrillose, annulus fibrillose, persistent

Bank by roadside amone mosses

Specimens examined

Costa Rica: San Jose, Rancho Redondo, 2500m, Dodge \&Thomas 5133 

Armiliaria

Plleus $1.5 \mathrm{~cm}$., surface pruinose to very slightly verrucose, scaly, margin thin,appendiculate with volva;warm buff, shading to ochraceous-tawny disc; gills adnate, close, white; stipe $3 \mathrm{~cm}$. \# $3 \mathrm{~mm}$., warm buff, fibrillose above; annulus ochraceous-tawny above, belonfverrucose, scaly, scales floccose at annulus.

Bank by roadside

Specimens examined:

Costa Rica, San Jose, Rancho Redondo, Alt. 220, Doage \$ Thomas 5135 


$$
\text { 1. }
$$


Armiliaria

Pileus flat, $5 \mathrm{~cm}$. with slight conical umbo, disc pyramidal, scaly, margin striate-sulcate, flesh thin, except on disc; gills white, ventricose, pink decurrent by a line, edges darker, close; stipe spongy, fleshy with fibrous cortex, cottony below; somewhat evanescent annulus, ending in bulb, $2 \mathrm{~cm} . \# 5 \mathrm{~mm}$. Mossy bank of roadside

\section{Specimens examined:}

Costa Rica, San Jose, Rancho Redondo, Alt. $2500 \mathrm{~m}$. Dodge \& Thomas 5235 


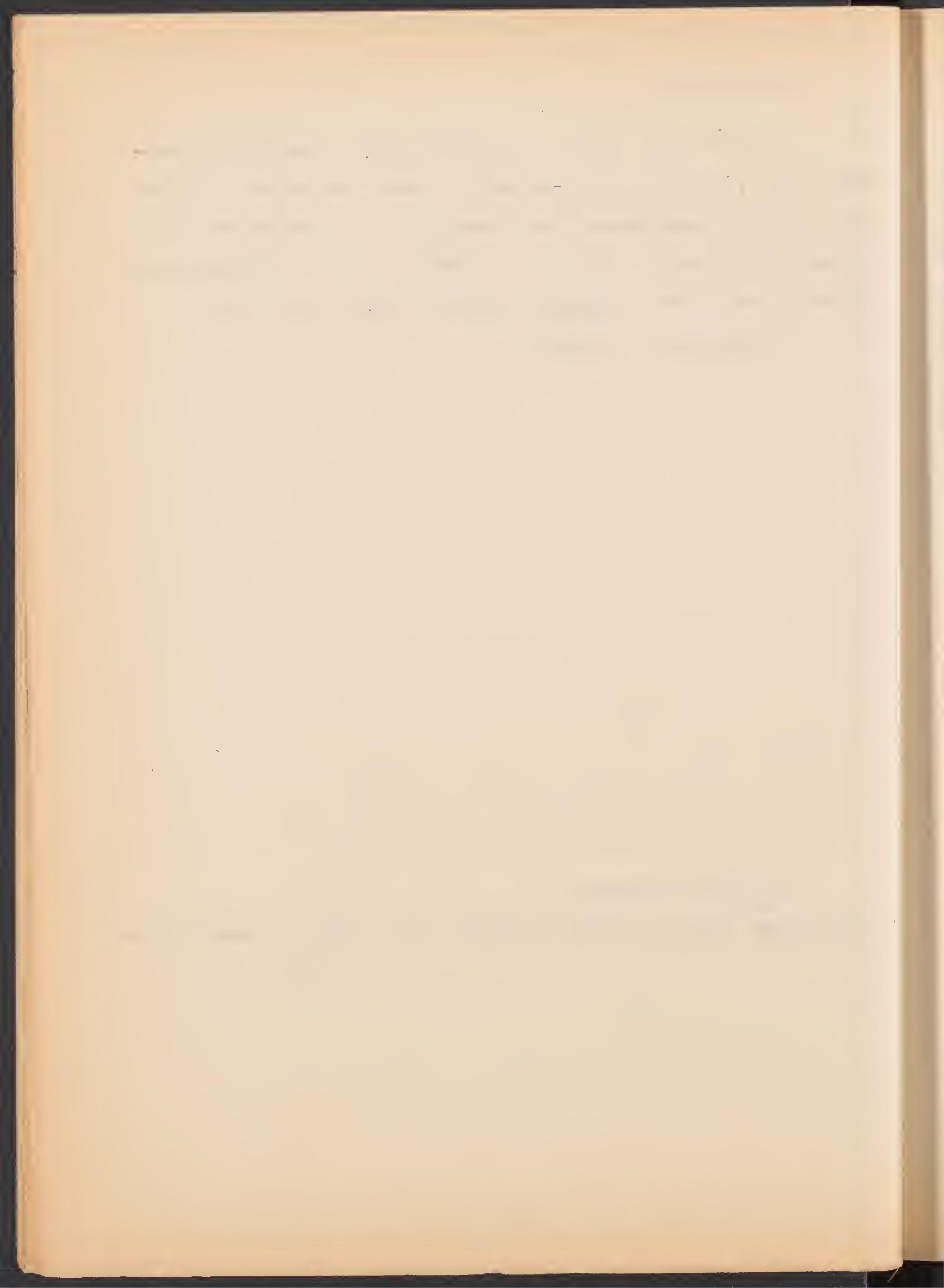


Armil Laria

Iopiota

pileus hemispheric, margin thin, unrolied, appendiculate up to $7 \mathrm{~mm}$. diam, surface pyramidal, warts like Elaph. variegatus but much finer, antimony yellow, flesh, medium thickness, a Itte lighter than surface; gills close, white, ventricose, sinuate-adnate; ring like Cortinariug_ but thiker; stipe tomentose above ring, below with warts like pileus, but larger and of looser texture, $2 \mathrm{~cm}$. \# $1,2 \mathrm{~mm}$. above ring, $2 \mathrm{~mm}$. below, ending in slight bulb

Bank of roadside

Specimens examined:

Costa Rica, San Jose, Rancho Redondo, ALt. $2250 \mathrm{~m}$. Dodge \& Thomas 5000 


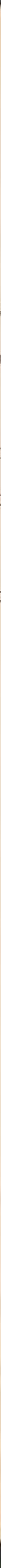

er

i

Her 
Armiliaria

Pileus not fully expanded, $2.5 \mathrm{~cm}$ in diameter, $1.5 \mathrm{~cm}$ tall, convex, with large broad umbo, densely strigose tomentose scaly an umbo, strigose scaly over rest; prouts boown, margin appendiculate; gills adnate to slight ly decurrent, close, broad, white; stipe $6 \mathrm{~cm} \times 5.5-12 \mathrm{~mm}$, ring slight perhaps evanescent in fully mature specimens, fibrillose spongy, drab gray with fusiform bulb, 12 mon tapering upward.

Heredia, Concordia, 1900 m., Goerger

$$
9-9-36
$$



P1leus expanded, $10 \mathrm{~cm}$., depressed, margins wavy, strlate, unrolled when young broady umbonate in depression, Mars brown, lighter toward margin, even, honey color, minutely fibriliose, scaly, flesh thin, except on disc; gills decurrent by a line, adnate, ochraceous-salmon, darker in age, narrow, close; stipe fibrous, stuffed, tapering downward, $6 \mathrm{~cm}$. $\hat{1} \hat{\mathrm{i}} \mathrm{mm}$ above, $\hat{5} \mathrm{~mm}$. belwo, silky, fibrous with floccose warts just below \& on underside of lacerate, fleshy, fibrous annulus.

Stump in potrero

Specimens examined:

Costa Rica, San Jose, Rancho Redo\$ndo, Alt. 2200向. Dodge \& Thomas 5043,5406 
Armillaria

Pileus expanded broadly umbonate, disc minutely scaly, cinnamon brown shading to cinnamon buff, margin striate sulcate; gills ginuate adnate broad, close, pale pinkish cinnamon; stipe fibrous, cottony below, somewhat evanescent annulus, tapering or equal $7 \times 1$ cm Formine fajry ring around old decayine stump

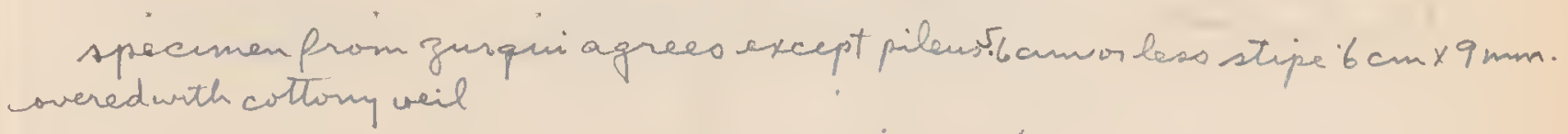

specimens examined

Costa Rica: San Jose, Rancho Redondo, N 2200 m Dodee\&Thomas 5408 Heredia: Cerro Centralde Zurqui. $1600 \mathrm{~m}$ DodgeValeriot Thomas 


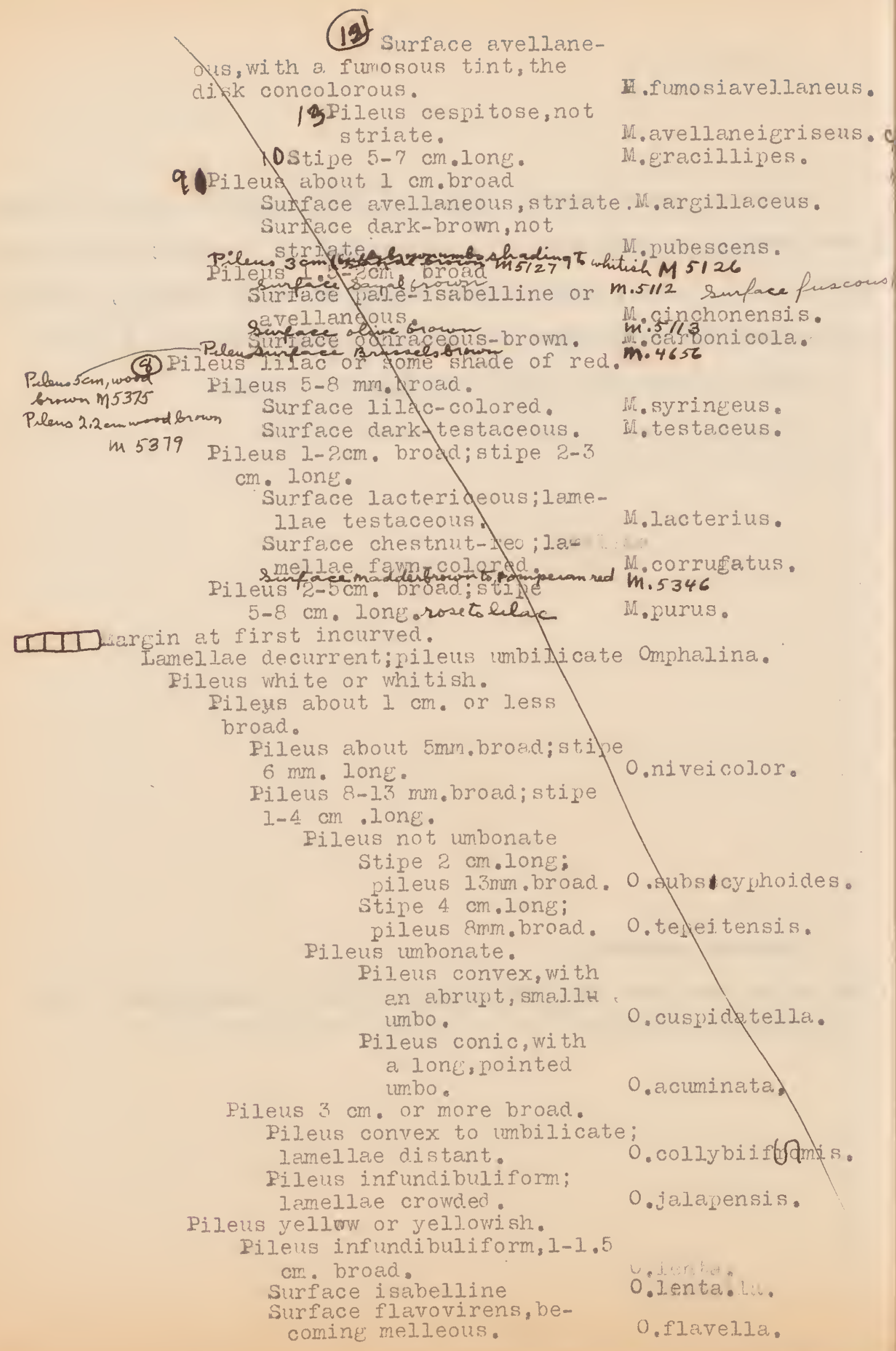




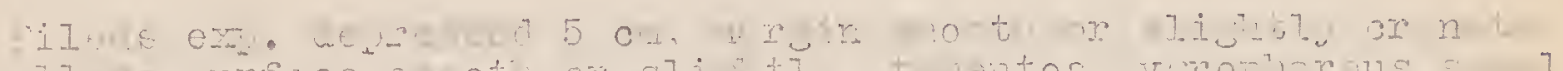

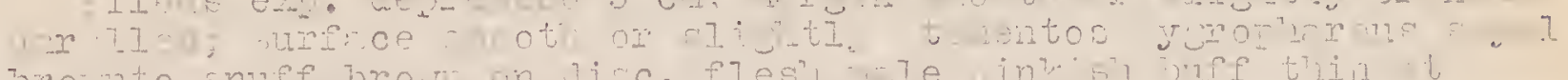

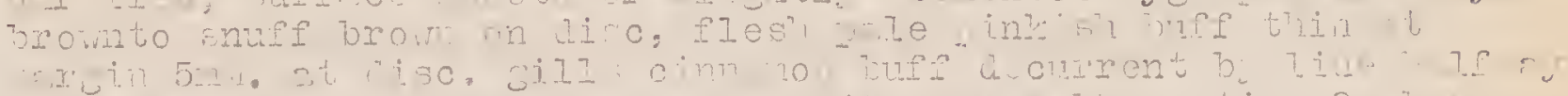

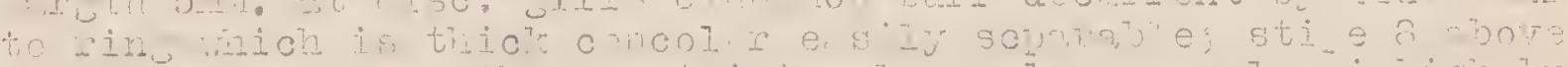

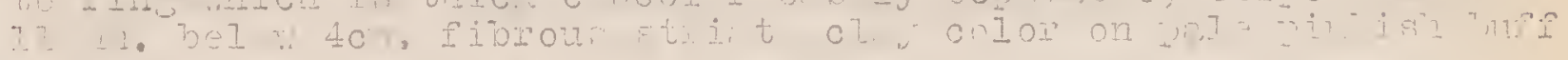
sroutins

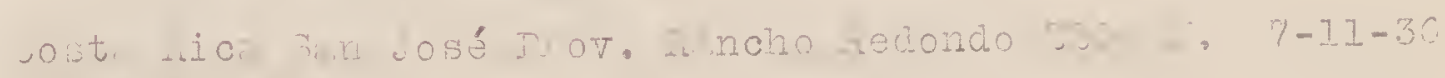
lod vocier 
Armiliaria

Plileus not fully expanded but convex, $2.5 \mathrm{~cm}$, very squarrose to pyrdmidal, scaly on disc, less so toward margin; gills close ventricose, sinuate-adnate, white, decurrent by Iine to annulus; stipe short, 3 \# I cm., white, cottony without becoming cinnamon,buff where handied.

Among mosses.

Specimens examined:

Cost:arica, San Jose, Rancho Redondo, Alt.2300 m. Dodge \& Thomas 

Armiliaria

Pratrolond

Pileus expanded $5 \mathrm{~cm}$.; margin thin,unrolled with remains: of partial ve1l; surface innate-scaly, appearing areolate, pinkiahcinnamon and sayal brown; gills, light plnkish-cinnamon, close, sinuate-adnate with slightly decurrent line about $2 \mathrm{~mm}$. to evanescent annulus; stipe, thlck, short, tapering upwara, white becoming yellow where handled, fibrillose-scaly, pale fleshy, $3.5 \# .7-$ $1.1 \mathrm{~cm}$.

on decayling 108

Specimens examined:

Costa Rica: San Jose,Rancho Redondo, Alt.2400m. Dodge \& Thomas 5257 

Armiliaria

Chaegta

New Genus.

Pileus verrucose on disc;flaccose-squamulose, margins, glabrous,-striate, orange-cinnamon to Mikado brown;gills, Itghtpinkish-cinnamon, darker eage;plane of gill shallow, reticulateporose as in young porold Merulius; annulus broad,persistent but thin;stipe fleshy, striate abovefannulus, floccose, striate below; airt adhereing to bulb as pseudovolva, $13 \mathrm{~cm}$ \#o6 mb., continuous with pileus.

Mossy bank.

\section{Specimens examined:}

Costa Rica:San Jose,Rancho Redondo Alt. 23-2400 m. Dodge \& Thomas 
Armillaria

Cortinarius

Pileus expanded $18 \mathrm{~mm}$. antimony-yellow with long conical, dark Indian-red sines, especially on disc; eills close,broad,adnate with decurrent tooth antimony-yellow; stipe fleshy.3.5 cm. \# $3 \mathrm{~mm}$. antimony-yellow above, whiter below; annulus cortinoid, evanescent; stipe fibrillose, splitting.

Specimensexamined:

Costa Rica, Iimon, Rose Hill, Alt.90 m. ,above Rio Pacuare, Catt, Dodge, \& Thomas 5540 
Bolbitius

Gomphidius?

Coprinus?

Pilelas campanulate, slichtiy umbonate, fibrillose, salmon to rufous, margin not striate; gills crowded, broad, free or nearly so, white at first, then brown and deliquescing;stipe concolorous, I cm \# I mm.,sliehtly fibrillose with remains of cortina formine inconspicuous ring.

Decayine $\log$.

Costa Rica, Limon, Rose Hil. above R.Pacuare, Catt, Dodge, \& thomas 5509 
Dileus $1.5-2 / \mathrm{cm}$, campanulate, merein inrolied, minutely tomentose, japkn reae on disc shadine to flech color et maruin; eills brown, deliciuescine, close, free; stige fragile, cartileginous, smooth, fJesh color above rxme, japan rose ring, white below, eloneating to $3-4 \mathrm{~cm}$.

Decayine stumps and logs in bananal.

specimen frou castilla quly 26 pleshoche shading to salmon, qulls mummy brown, stipe whte runq evanoacent. Specimen fron R. Sandalo liqht salmon ounge

Costa Rica:Iimon, Marta $20 \mathrm{~m}$. Dodee 7306; Castilla quly 2 Puntarenas, Osa, R Sándalo 5m Dodge tgoerger ang 24, Sept I 


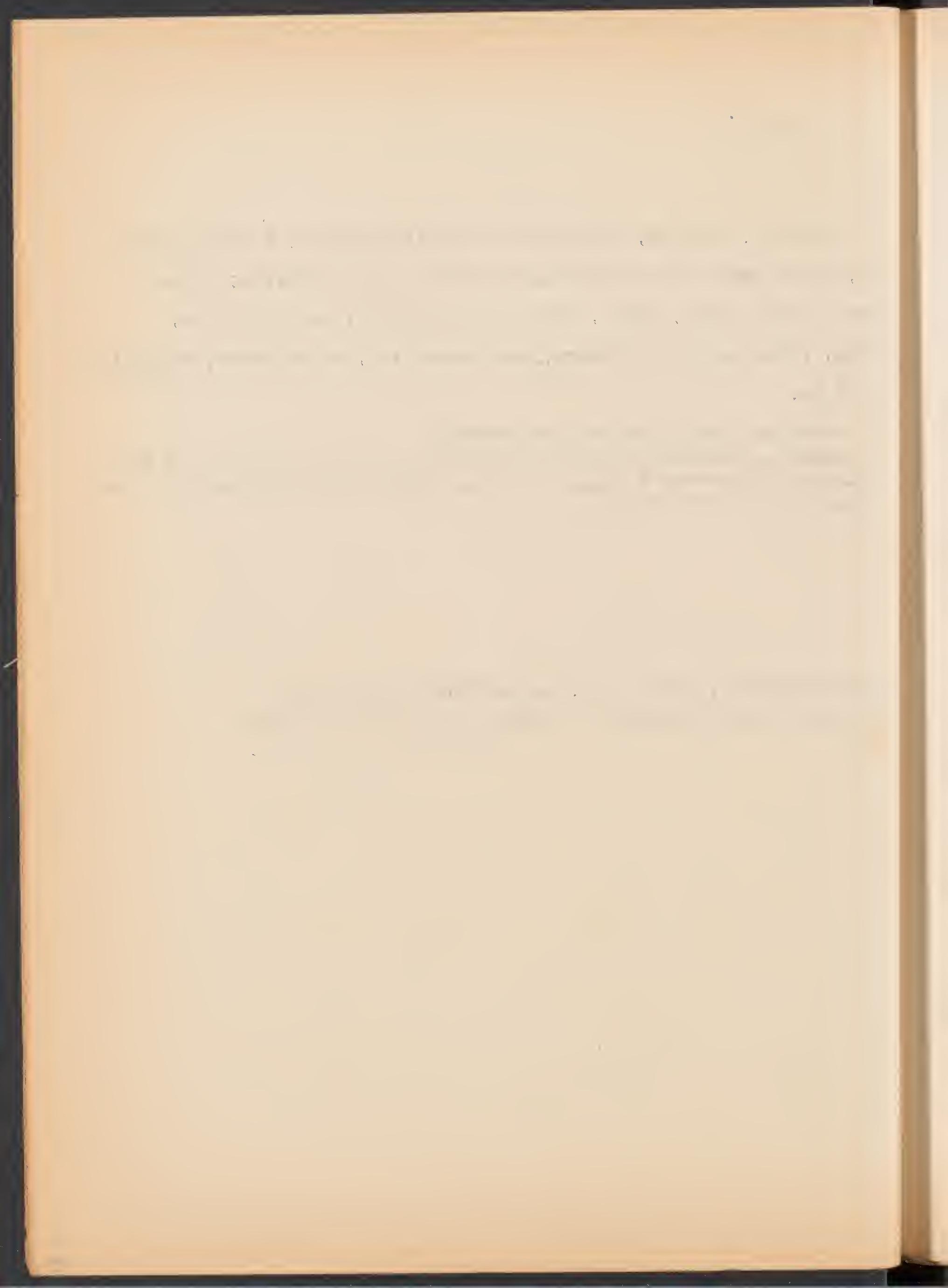


Boletus

see painting. Surface cracked minutely velvety; tubes large angular, not changing color when wounded, mouths not different color: Glesh with faint pankish tint, dereloping slowly after cutting; stipe deep brown, broably starting to decay since tubes and part of the surface of cap show whitish mycelium

Specimens examined:

Costa Rica: San Jose, Rancho Redondo, $2230 \mathrm{~m}$, Dodge \&Thomas 4985 


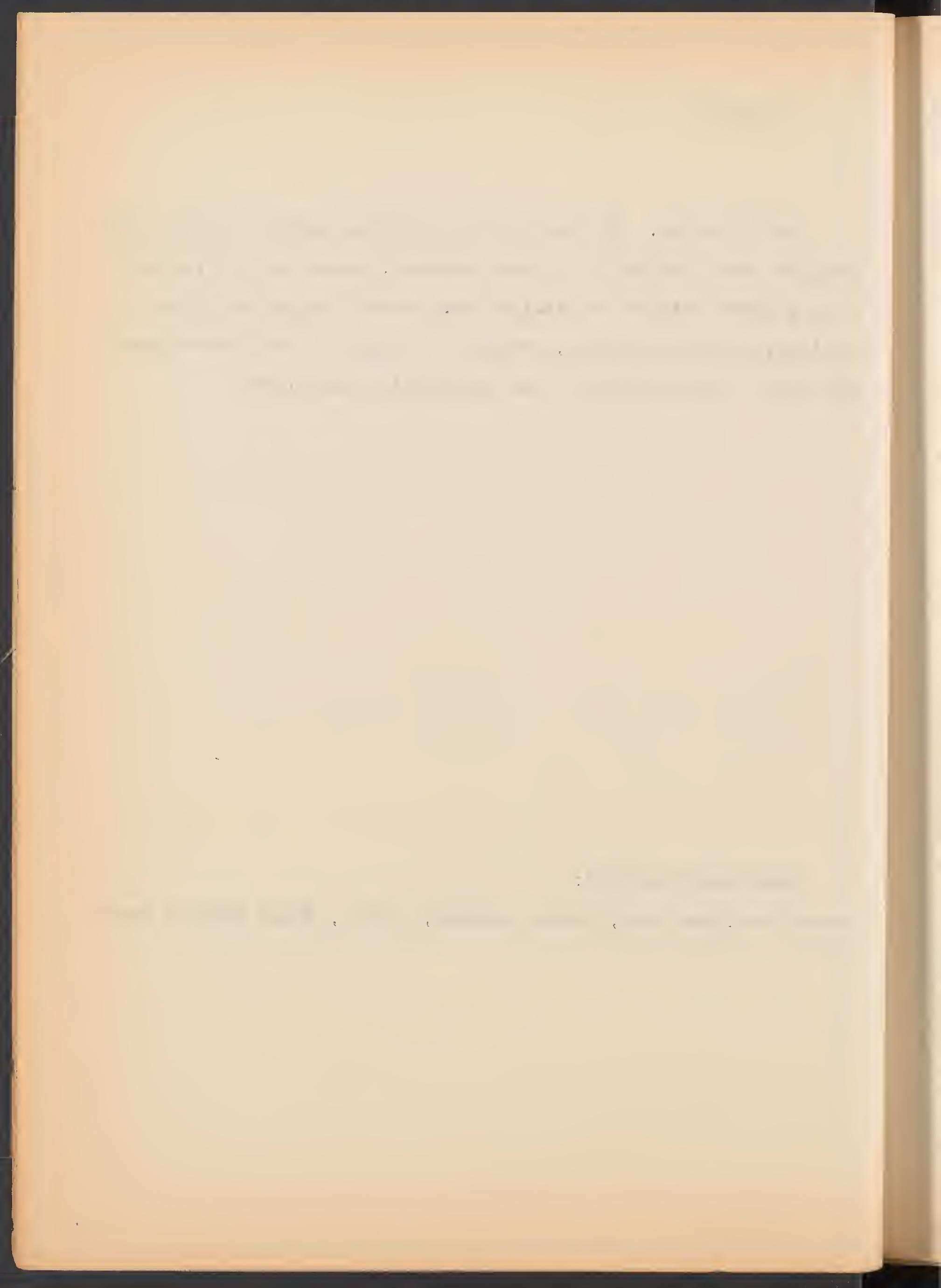


Boletus

Pileus $1.7 \mathrm{~cm} \mathrm{~cm}$, minutely verrucose, velvety, orange rufous or darker, convex, flesh thick on disc thin toward margin; tubes white, depressed around stipe; stipe floccose tomentose, concolor taperingupward $3.5 \mathrm{~cm} \times 3-5 \mathrm{~mm}$

Mossy bank by roadside

$S_{n}$ ecimens examined:

BCosta Rica:San Jose, Rancho Redondo $2150 \mathrm{~m}$ Dodge \& Thomas 5120 
Boletros

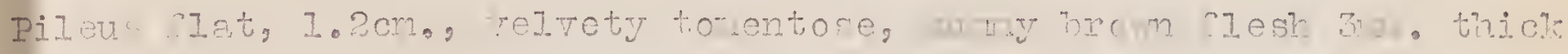

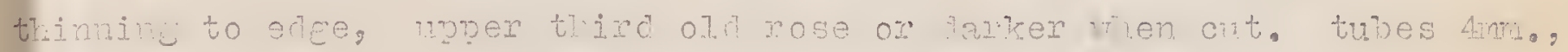
yello:, worths of tribes at marein veined old rose

Stipe rexy anort, bromen off, .6X?m.

Tosay rondide hanks

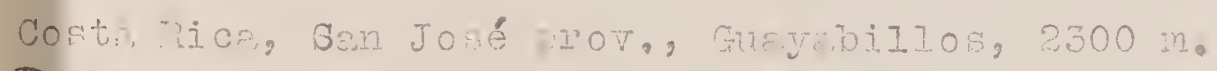
Dodge + Goerger 
52

rectus

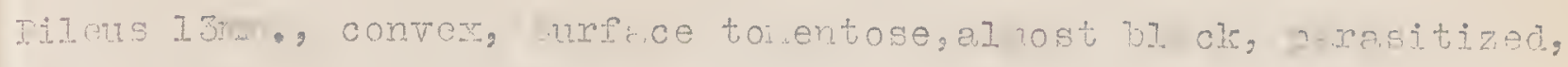

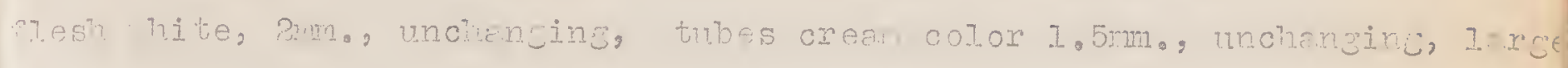

ny il I

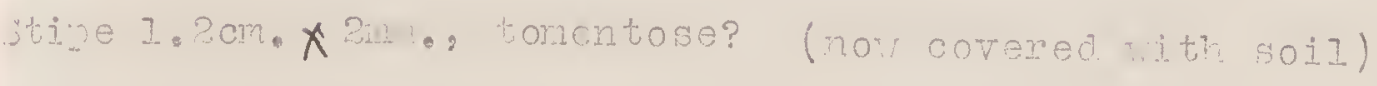

Mossy roadside bant

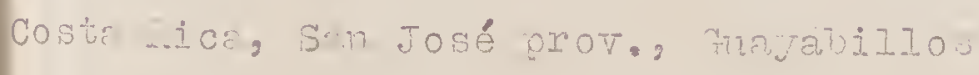

$7-26-7 n$

Dodge + Goerger 


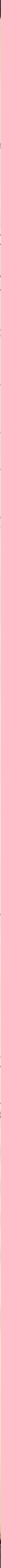


01 ten:

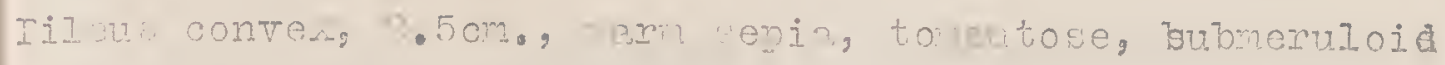

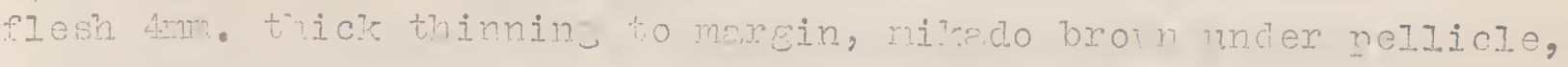
waforr yeliow unchncinúgtubes dare te cheprecsed rounc stipe, limo meen,

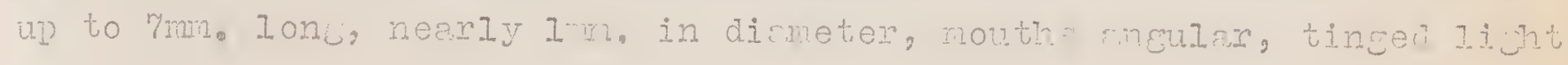
jusper red.

Stipe tinged li ilt $\mathrm{y}$ sper red, tomentose, $3 \mathrm{~cm} \times 6$-4mm, tapering dointiand.

on m, sy bank

Coste Nicn, Gan José prov, rancho redondo mecr britge $7-13-36$ Dodce i Goenuen 
Cantharellus

Pileug convex, flesh thick, $2 \mathrm{~cm}$, orange buff, spongy fibrillose; decurrent gilis narrow, distant, with anastomosing merulioid cross gills, orange thick; stipe $6.5 \mathrm{~cm} \times 3 \mathrm{~mm}$, fleshy trexpme tapering downward, concolor

Heredia Chahuites, $1600 \mathrm{~m}$., Goerger 
Cantharellus

Pileus $4 \mathrm{~cm}$ (both pilei in poor condition but apparently buff) infundibuliform; gills orange buff.

Specimens examined:

Costa Rica: San Jose, Rancho Redondo, $2250 \mathrm{~m}$, Dodee \&Thomas 5132 


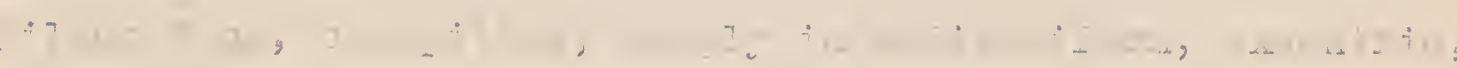

2.

07.

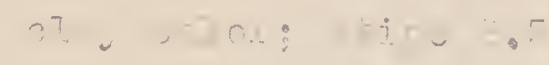


- 


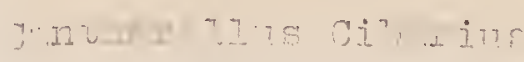

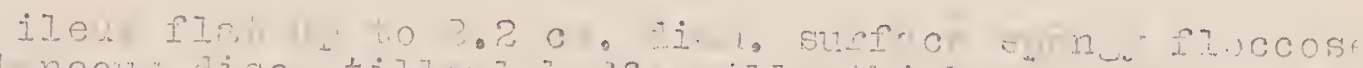

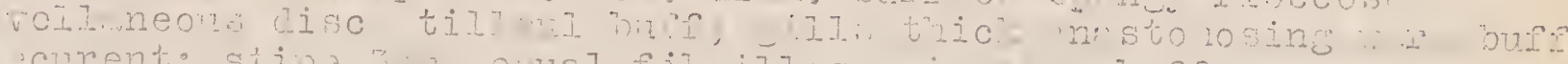

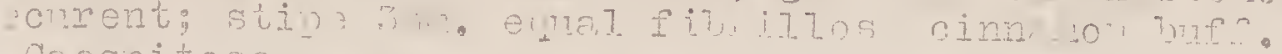

Crespitose

Terrestrial hebredi forest

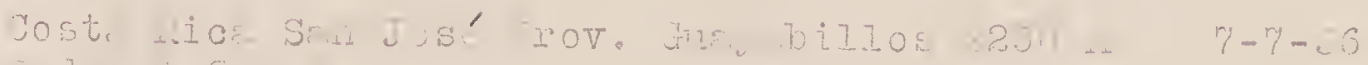
nace torger 
Chamseota

pileus $5.2 \mathrm{~cm}$ diam, fibrillose, bibulous, deply sulcate-striate over gills, disc fuscousto fuscous black surrounded by zone cream withsmall fuscous scales, margin light vinaceous fawn; eills free, ventricose, close, light salmon orance; stipe fleshy, taperine upward $9 \mathrm{~cm} \times 4-9 \mathrm{~mm}$, silky fibrillose, white, rine apressed, inconspicuous, erect $3.5 \mathrm{~cm}$ below apex, no sigh of rolva

Costa Rica: Puntarenas, Osa, Estero Sandalo 5-20 m Dodee 7665 


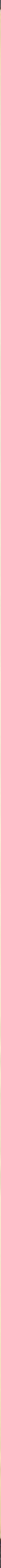


Clarkeinda

Pileus $3.2 \mathrm{~cm}$, convex, viscid, margin stiate, dresden brown on disc shadingto buckthorn brown; gills broad, subdistant, sinuateadnate decurrent by a line, dark brown; stipe reticulate verrucose above thick persistent annulus, below fibrillouse reticulate with remains of partial reil, hollow, somewhat crooked, ending scte in bulb with somewhat filamentous eleified rolva $9 \mathrm{~cm} \times 5-9 \mathrm{~mm}$ On earth near rotting $l o g$ in potrero

\section{Specimens examined}

Costa Rica: San Jose, Finca de Alfredo Volio above Cabeza de Vaca 2700m Rancho Redondo, Doddge \& Thomas 5372 


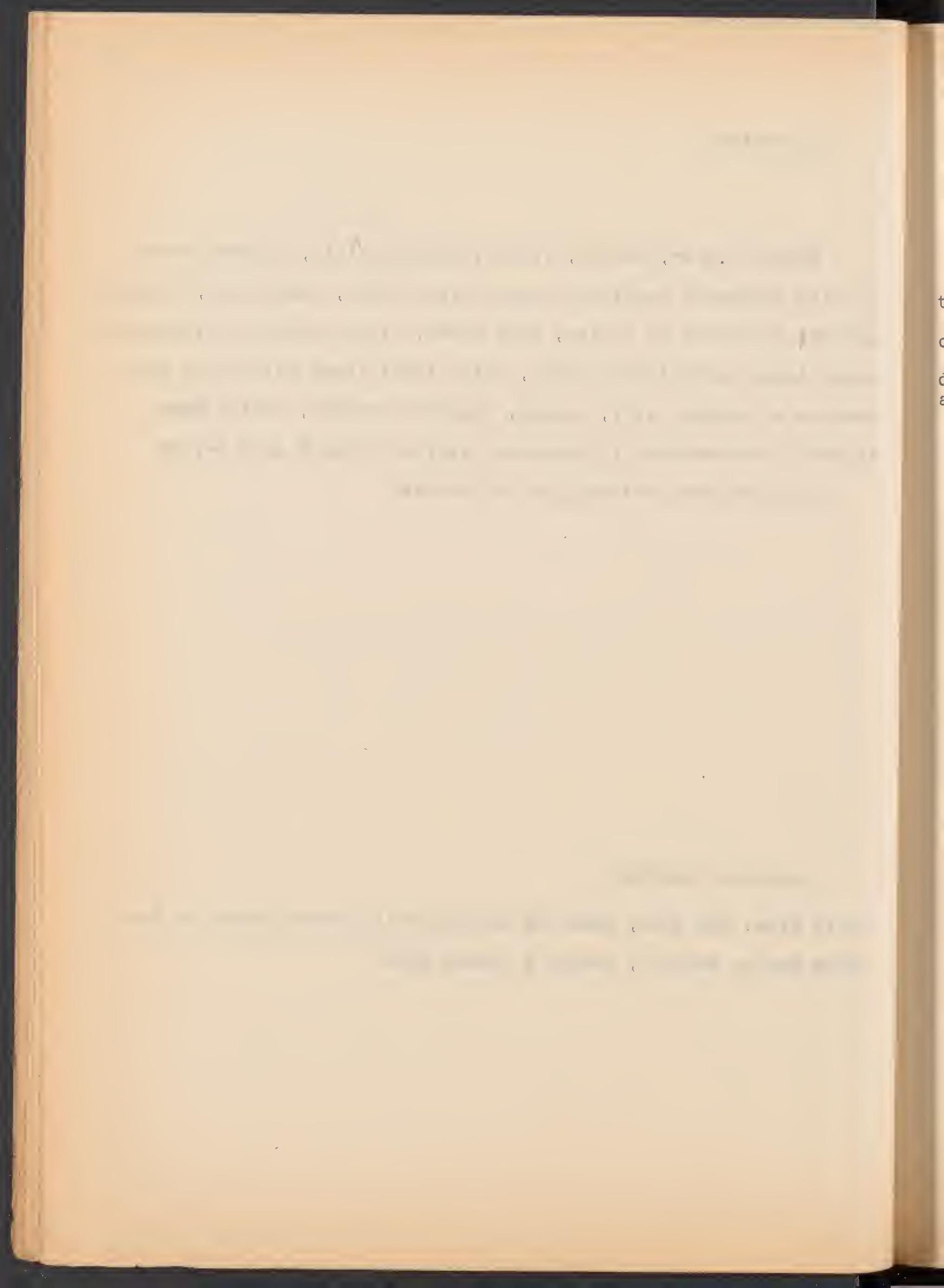


Iglesias Valerionis

Pileus hemispheric, marein slightly inrolled $2.5 \mathrm{~cm}$, clay color to cartridge buff at margin, smooth shining but not viscid; gills close, broad, sinuate adnate, oljve brown; stipe slender, tapering do downward with bulb at base $55 \times 4-5 \mathrm{~mm}$ white stained greenish(by algal growth?)

annulus collapsed about stipe, thin, black

Incow dung in potrero

Specimens examined:

Costa Rica: Heredia, Cerro Central de $\operatorname{Zurc}_{1} u i 1530 \mathrm{~m}$, Dodee, Valerio \& Thomas 6135 
Clarkejnda ?

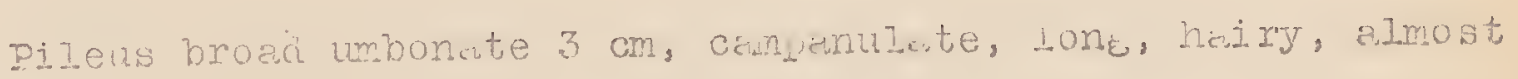
Wracty, edce lone fimbricte, a wencicujite, whonate, mikado krown to tamy orive, silittine easily; eills broad cuttulate free cimismon brow, close; stipe cottony tomentose, white above cortinoid subevanescent annulus, below towny olive, fivrijlowe, $9 \mathrm{~cm}$ x $5 \mathrm{~mm}$ ending in bujb with suivel.minous volve

so j.]. neir decaying wood

Bipecinens exwined:

Costa Rica: Sean Jose, Rincilo Recondo $2700 \mathrm{~m}$, Dodete dnomas 5336 

Clarkeinda

Pileus $3.7 \mathrm{~cm}$, convex with conical umbo, riscid with punctate remaj.rs of universal veil, tawny with massicot yellow mareins, the latter tawny punctate from remains of veil; gills adnexed or sinuate adnate decurrent by a line old gold becoming purple brown, b broad, subdistant; stipe white punctate pruinose above the broad below squarrose scaly, fibrillose with lacerate persistent annulus,/a few light tomentose remains of partial reil ending in a small bulr with gelified rolva which now includes particles of soil in the gel

Among grasses in potrero

Specimens examined

Costa Rica : San Jose, Guayabillos 2700 m Rancho redondo Dodge Dodge \& Thomas 5370 


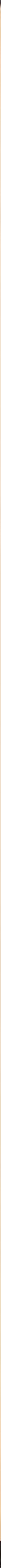


Clankeunda??

titroupharar

Politibia purpte-spored
Psilocybe

Pileus expanded, convex, viscld after disapperance of shaggy, squamose, universal. ve1l, russet disc, shading to antimonyyellow, $3.5 \mathrm{~cm}$; gills adnate, sinuate, ventricose, close; flesh thick on broad umbo, yellowish; stipe hollow, cartillaginous, shag8y, belwo remains of cortina, some of which remains attached to margin of pileus, russet after handing, bulbous, dirt clinging as a pseudovolva.

Specimens examined:

Costa Rica, San Jose, Rancho Redondo, Alt. 25-2650, Dodge \& Thomas. 5160 


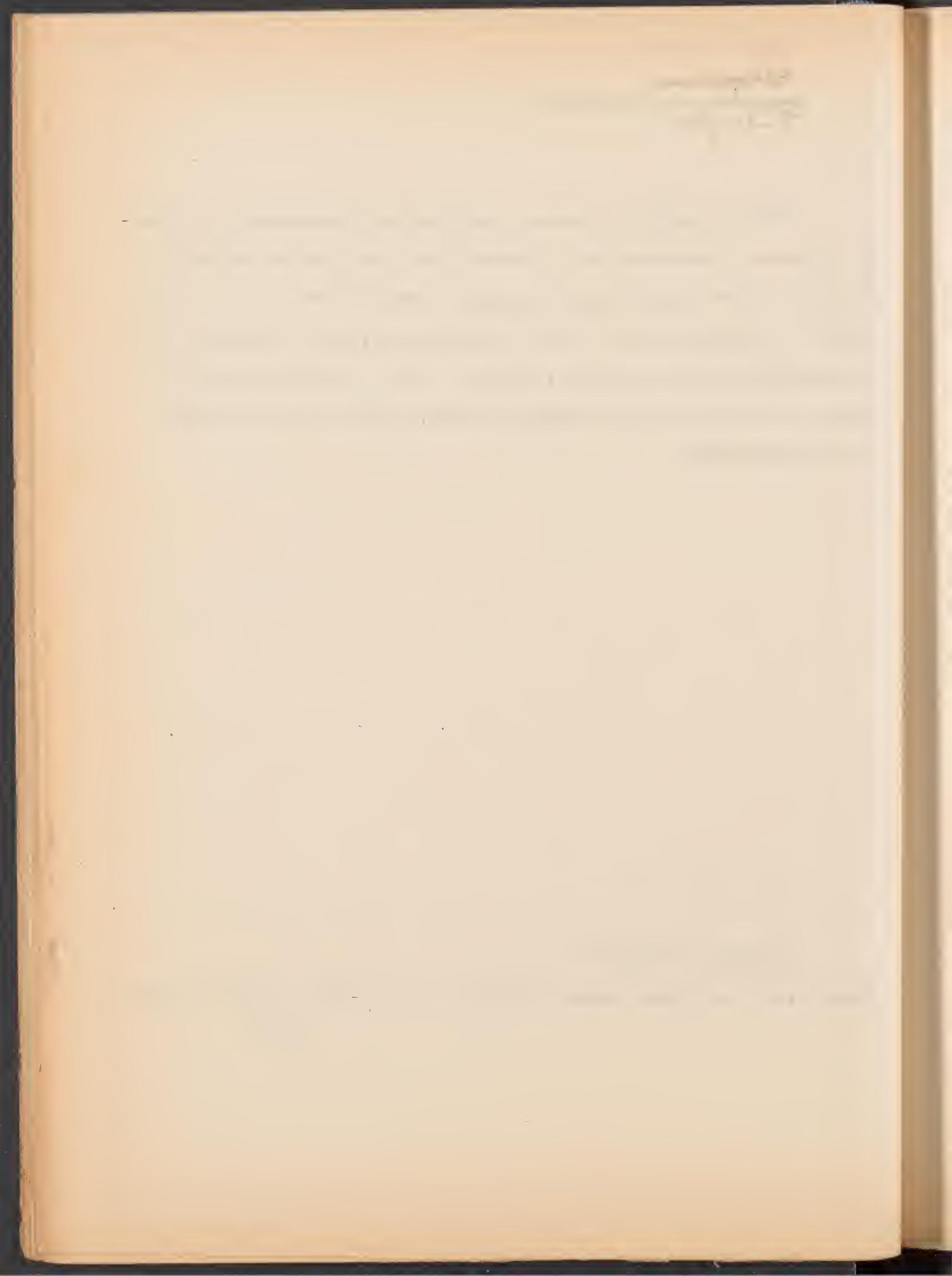


Cli to cybe

Pileus small, about $4 \mathrm{~cm}$ diam, disc viscit, smooth, clay color margin fibrillose, flat, flesh thin, cinnamon buff consisting of gelatinous pellicle, a compact watery layer with a subgelatinous layer beneeth, the fleb stipe layer extends up over the gills beneath th e subelatinous layer, while the cottgn stuffing of the stipe fills the interior and is somewhat riddled by insects; gills adnate, margins serrate, eroded, subfloccose, white; stipe covered with floccose scaly remajns of veil, about $8 \times 1 \mathrm{~cm}$.

\section{Specimens examined}

Costa Rica: SanJose, Rancho Redondo, $2250 \mathrm{~m}$, Dodee \& Thomas 5020 
Clitocybe nearBroadwayi

Pileus infundibuliform, $4 \mathrm{~cm}$, striate, sulcate, marein sliehtly inrolled, tilleul buff; gills warm buff, decurrent, narrow, distant; stipe $4 \mathrm{~cm} \times 85-4 \mathrm{~mm}$ white slightly tomentose

Costa Rica: Puntarenas, Osa, near Rio Terrones 5-20 m Dodge 7735 


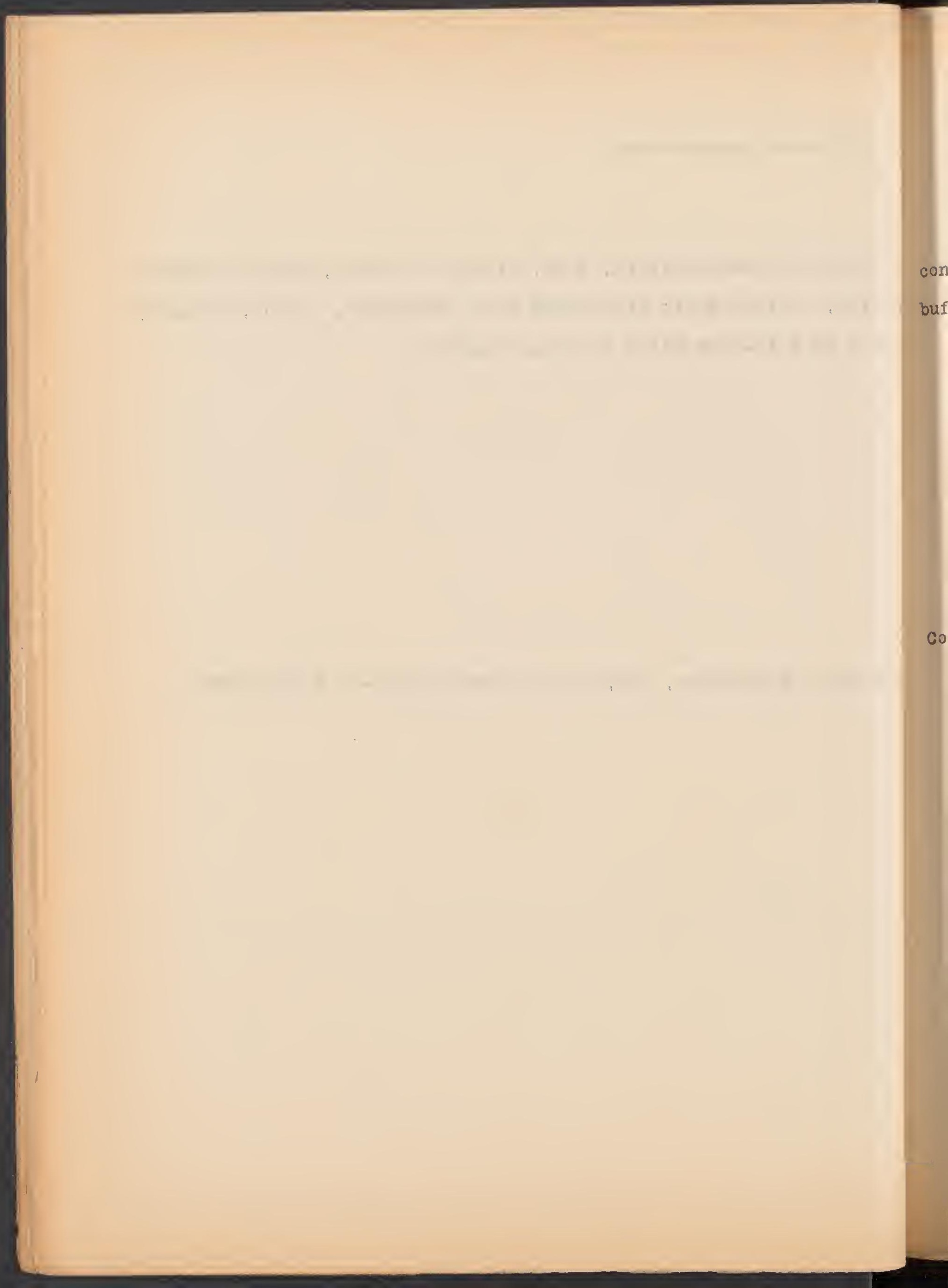


pileus $3-4 \mathrm{~cm}$, cinnamon brown topale ochraceous buff, plane, convex, umbilicate;margin faintly striate; gills pale ochraceous buff, narrow, close, decurrent; stire $4 \mathrm{~cm} \times 4 \mathrm{~mm}$, concolorous

Costa Rica: Puntarenas, Osa, Fsterosandalo 5-20 m Dodee 7698 


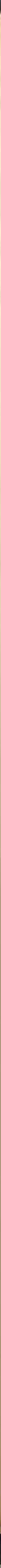


cli tocybe

Pileus flat with sharp umbo $15 \mathrm{~cm}$ smooth, subtomentose, pinkish sinnamon, umbo sayal brown, $\mathbf{x}$ flesh thick, gills braod decur rent subdistant, thin, light pinkish cinnamon; stipe fleshy, 3.5 cm $x$ $4 \mathrm{~mm}$, light pinkish cinnamon, subtomentose

Among grasses in potrero $2260 \mathrm{~m}$

Specimens examined:

Costa Rica: SanJose, Rancho Redondo, $2260 \mathrm{~m}$. Rruth Dodge \& Thomas 5266 Dxx 


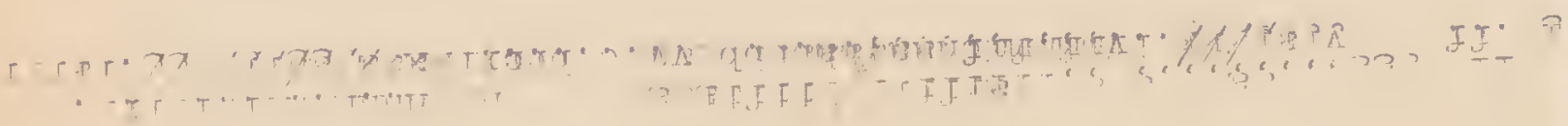




\section{Cli tocybe}

Pileus $7 \mathrm{~cm}$ deeply infundibuliform, sulcate, russet shading to light buff; context very thin; gills decurrent, broad, subdistant, white with cadmium yellow edges; stipe short fleshy, hyerophanous, $2 \times 1 \mathrm{~cm}$

On decaying, mossy log

Specimens examined:

Costa Rica: San Jose, Rancho Redondo, 2400m Dodge \& Thomas 5373 
Cli tocybe

Pileus \& stipe antimony yellow; gills slightly decurrent, wh white.

Amone erass in potrero

Specimens examined

Costa Rica: San Jose, Rancho Redondo, 2200m, Dodge \& Thomas 5100 


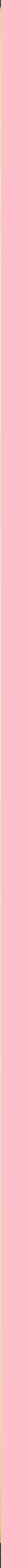


Clit tocybe

Pileus convex, slightly umbillicate, white, subhyerophanous, $2.2 \mathrm{~cm}$ diameter, flesh white, thin except on disc; eills white, decurrent, broad, close; stipe tapering downward $2 \mathrm{~cm} \times 3 \mathrm{~mm}$ fibrillose silky, hyerophanous, pruinose above Rotten log

Specimens examined

Costa Rica: San Jose, Rancho Redondo $2300 \mathrm{~m}$, Dodge \& Thomas 5117 
CIitocybe n sp.

Pilei cespitose, convex to flat, umbilicate, margin floccose, inrolled, $2.5 \mathrm{~cm}$., white, hyerophanous with pale j.sabelline tints, smooth dull; gills decurrent,close, naize-yellow; stipe white, $2.5 \mathrm{~cm}$. \# $6 \mathrm{mn}$. fleshy.

Dead Iog.

\section{Specimens examined:}

Costa Rica, Limon, above Rio Siquirres, AIt. 140-200m.. Catt, Dodge \& Thomas 5527 



\section{c.is tocybe}

pileus densely cespitose, epixylous, convex, maring, umbilicate, smooth, chalky white, upto $5 \mathrm{~cm}$ diam. eills narrow, close decurrent white, stipe white smooth, equal, $2.5 \mathrm{~cm} \times 3.5 \mathrm{~mm}$

Rottingl.og in bananal

CostaRica: Isimon, Marta, $20 \mathrm{~m}$. Dodee 


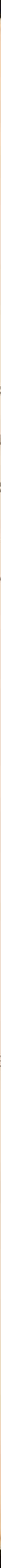


Clitocybe nearest testaceoflava

Dilei densej cespitase, thereby distorted and stipe awearine ccentric to almost lateral but marginate behind; ur to $3.5 \mathrm{~cm}$ diam. jinkish buff to pinkish cinnaxon, umbij.jcete, f'Jesh thick; éijJs very decurrent cuite thin, comparatively narrow, arpicot huff; stipe hick, fleshy distorted, seashell pink $1 . \mathrm{cm}$ djam about $1 \mathrm{~cm}$ lone tam perine slightly downward, flesh seashell jink or darker. decaying J.oe in bananaj.

Coster Rica: Limon, Marta $20 \mathrm{~m}$., Dodge 7218 of Costrlla 7-28-36. 
C1:

11
1
8
8

80 ?

$7 \mathrm{cr}$

$4 \mathrm{~cm}$

on

Co

Do 
9't

$61 j \div 0$ yoe

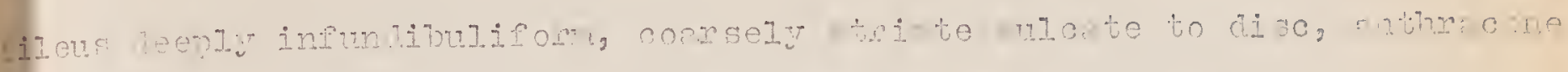

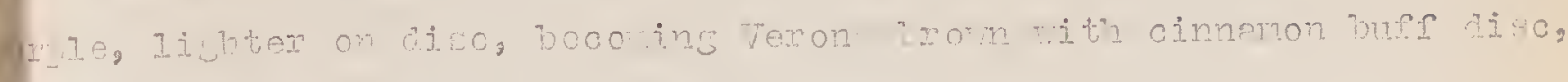

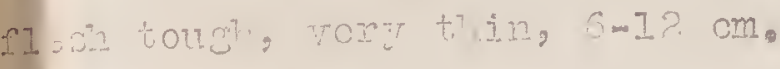

G'Jus nerror, arbdistant, decurrent, Iight winsceous Iilac

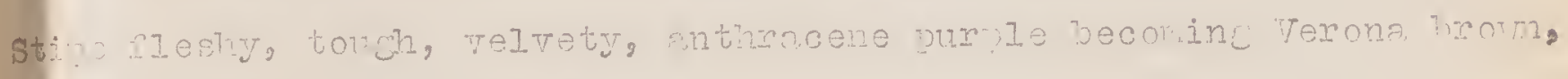

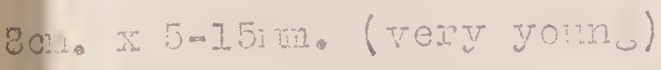

7on. $:$ 9m (purnze specimen)

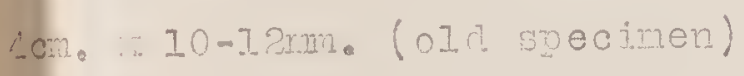

on dec.uil Ios

Cosur Rice, mutarenas nyov, osng R, Séndalo, I-5m. $-4-36$ Do je is goercer 


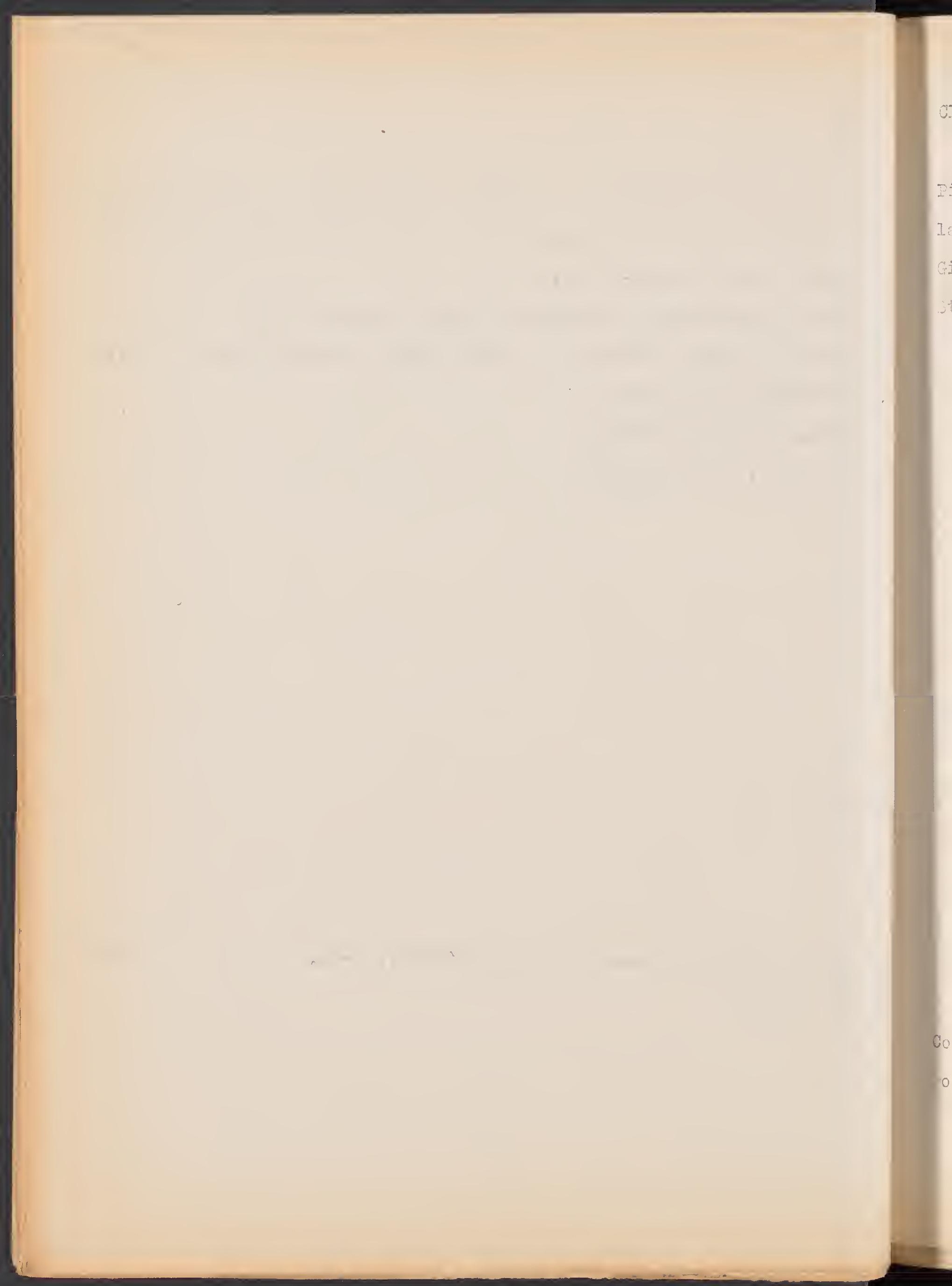


clit tocybe

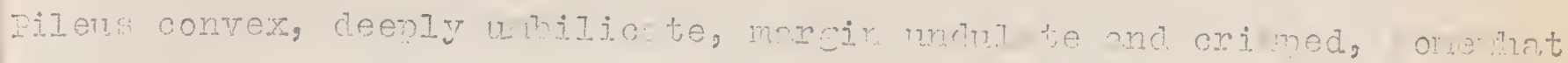

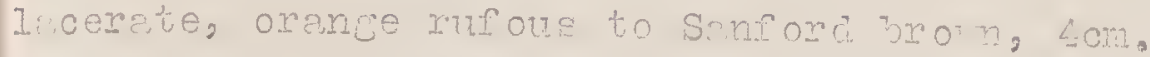

Gills hite, subcistant, decurcent, norrou

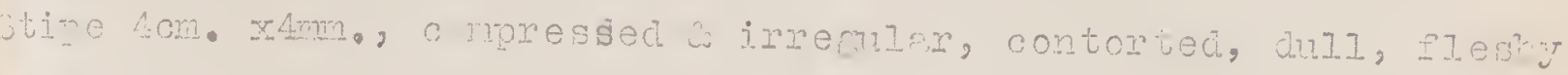

Costr, ich, muntorenne prov, Osa, R. S'ncalo, I-5m, $8-37-36$ olje coreger 
citocine n. p.

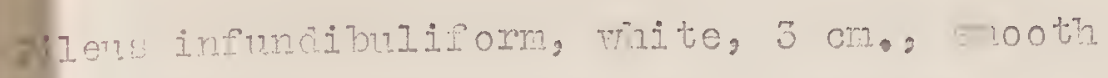

gills decurrent, white, close, narrow

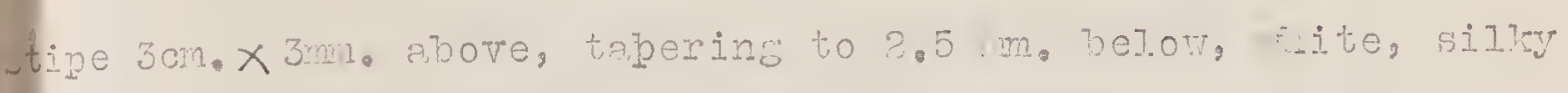

Corta sica, Linon prov., Castiza Parm, 20m. 
Iilevs vinaceous-rufous, leeply infundibuliform, fibrillose, $1.3 \mathrm{cin}$ in sineter

Gils nassjoot yellow, nerrow, crowded, decurrent

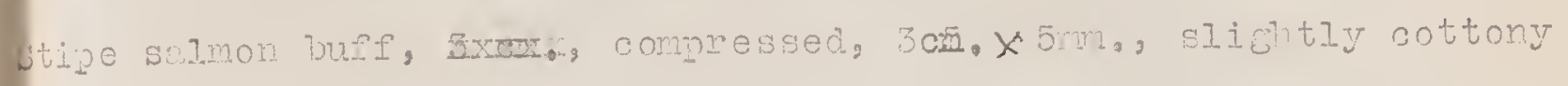

Leer unld in palin forest

Dosta Rica, Iinon prove, Castirl sarm, $20 \mathrm{~m}$ $7-28-36$ 


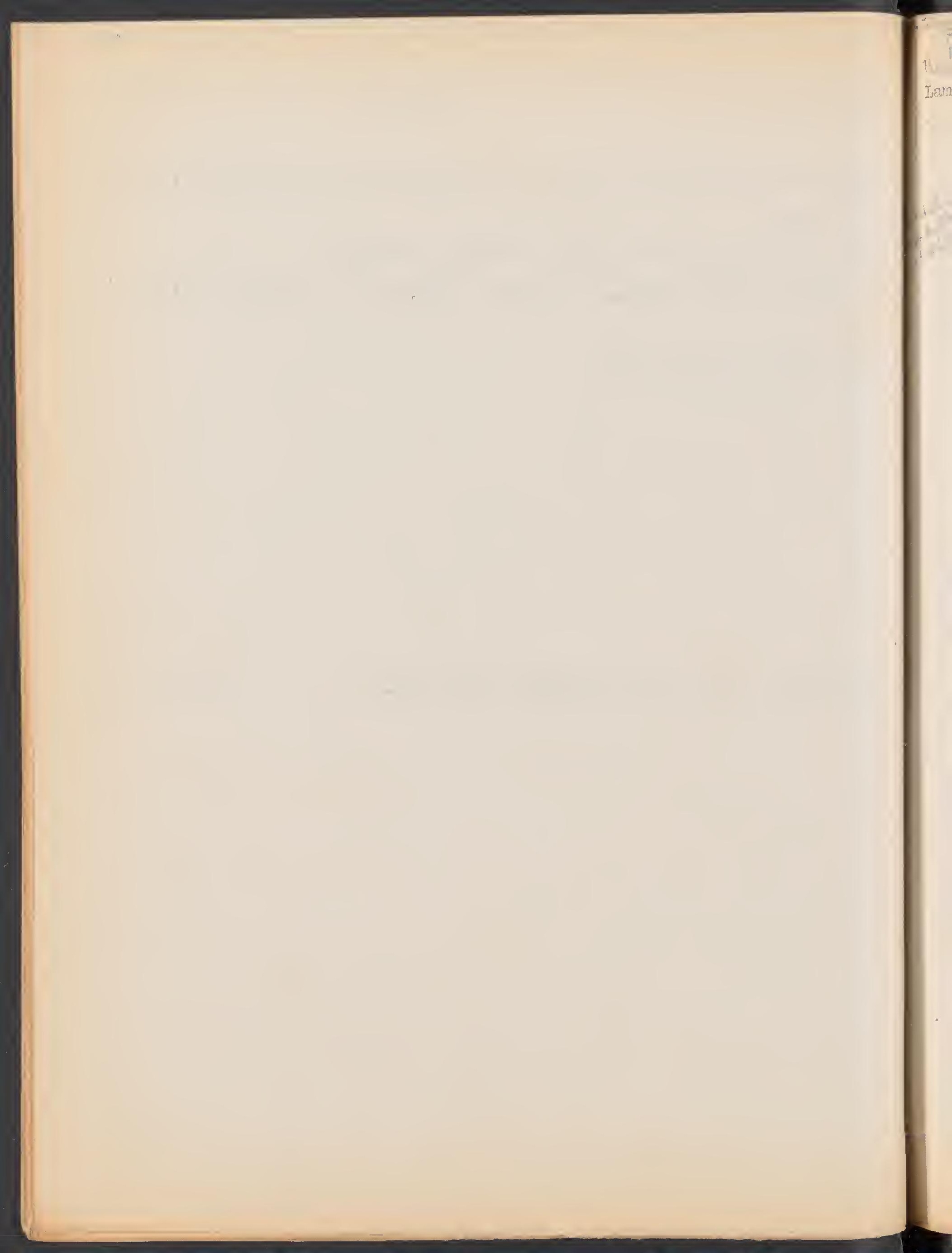




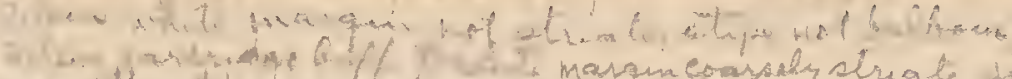

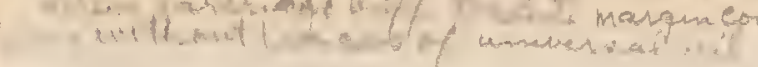

Iarnellae adnate

Pileus viscid or at least moist and glutinous

* Pileus pure white

Pileus colored(9-19)

Stpe viscid

Stive shining"

e $-\infty, 23$

Stipe velvety or tomentose

Irarein striate, vileus chestnut

Margin smooth

COJIYBIA

pizeus $4 \mathrm{cra}$, Sanford brown to raw sienna; stine $6 \mathrm{~cm} \times 2.5 \mathrm{~m}$ xanthine orange to Argus brown

Pileus $2-2.5$ crn stipe short

$$
\text { C. } 8-15
$$

pileus ochraceous orange to lanlée yellow

$$
\text { stipe } 2 \mathrm{~cm} \times 2 \mathrm{~mm} \quad \mathrm{C}, 7-16
$$

Pieleus amber brow to antimony yellow

$$
\text { stine } 1.3 \mathrm{~cm} \times 2.5 \mathrm{~mm} \quad \mathrm{C} .5308-5311 \text { incl. }
$$

Pileus pale olive buff to mite, merely clutinous, sinininc, margin strifte over fills; stipe $3 \mathrm{~cm} x 4 \mathrm{~mm}$ silky, compressed.

Pileus velvety, tomentose, pcaly or lacerate C. ITevermann

Pileus lacerate, nas brown with proninent conical umbo

Disc with pyramidal scales

$$
\text { C. } 7-10
$$

Pileus Prouts brom, stipe tomentose

Pileus onion shin pink, stips silky

$$
\text { C. } 7-17
$$

Pileus lignt ochraceous salion to russet, stipe flnccose

Pieleus tomentose scaly to floccose

Cuticle seprrable, pileus white
Stipe white
Stipe cinnemong buff with Seccardo's umber scales

Cuticle not separable

$$
\text { C. } 447 \text { I. }
$$

PIent wholly wite, disc slightly denressed

pilleus some shade of yellow or hrown

$$
\text { C. } 911 .
$$

Stipe white, snon blackening gilous buckthorn brown on a and

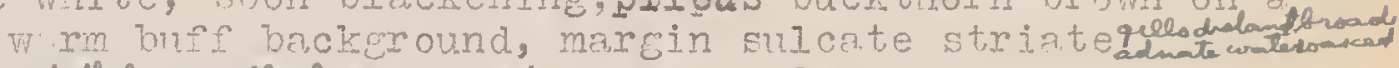

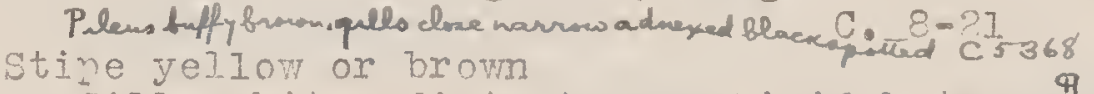

GiIIs white, distant, merulioid bet een, stipe moluy buenthombum

$$
\text { e.soton C. } 8-25
$$

Gilis antimony rellom, crowded, narrow

Stipesotatore tratulline

$$
\text { C. } 5.13
$$

Gills Marles yellow, subdistant, broad

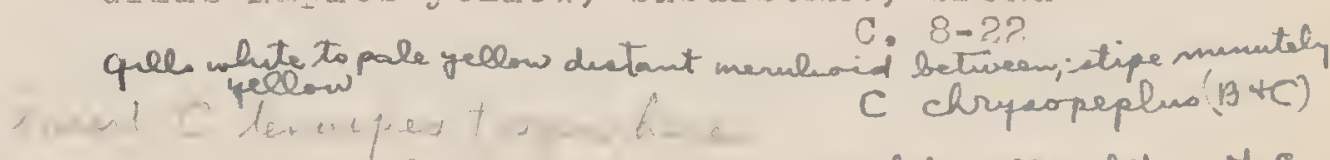

Pileus malogany ned to chestunt, umbonate, shpe white qulls white with Engleah red alge C 5028 

and inaturn to

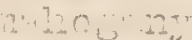

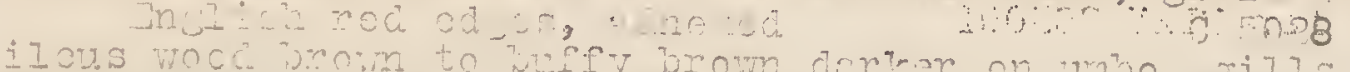

adnered subdistant

o. 5533

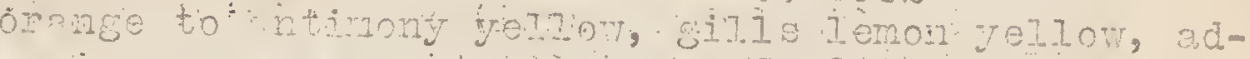
nate, cioso

jleus nntinony yeliow to yellow ochre, wijlz wite, anste,crowded

Dileus convex, not umbilicate

C. $51 ? 28$

ileus ninkish buff ard cinnamon buff, wtine thite noore, chestnut

$$
\text { or cirker below C. } 9-11
$$

Ieng buclethorn brom or drrkex, otime nosxly black

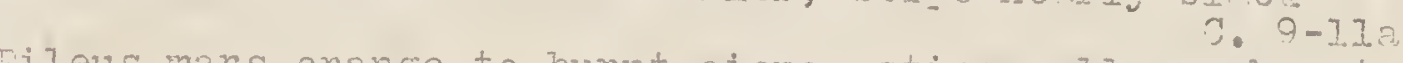

Fileng mars orange to burnt wienc, stino yeIInW oclire to ul ok

Stine velvoty, magins strifte

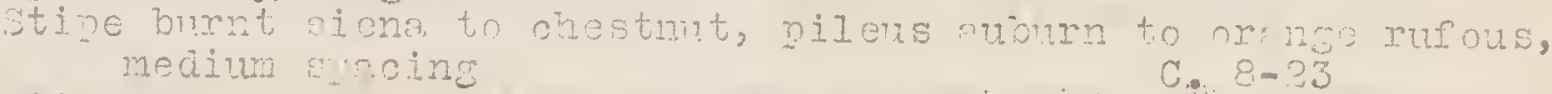

$\operatorname{ci}[1$

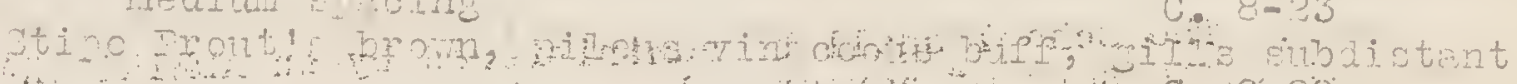

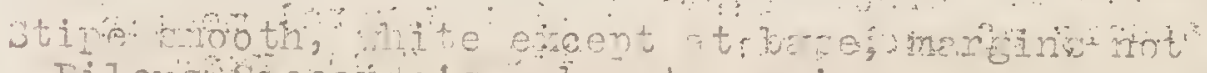

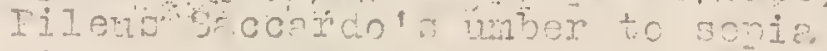

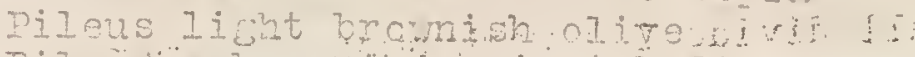

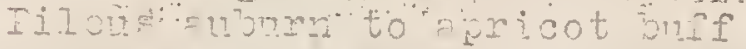

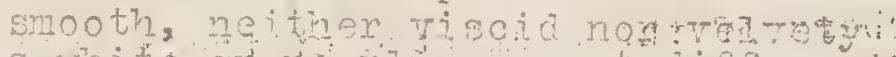

$8 \cdot 8-28$

wite

ilous umbonte

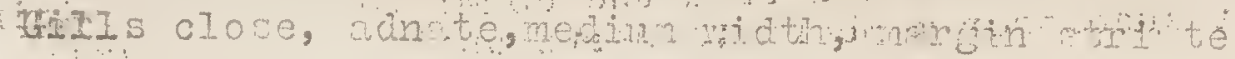

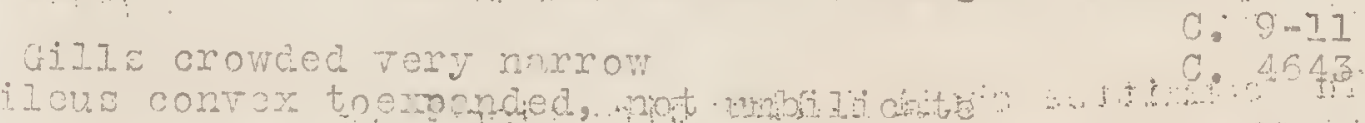

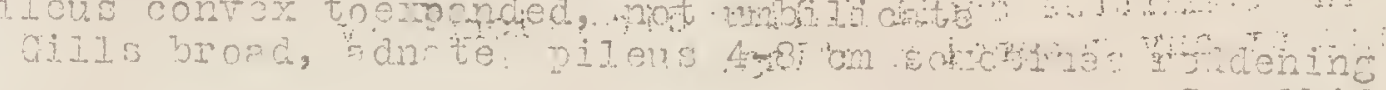

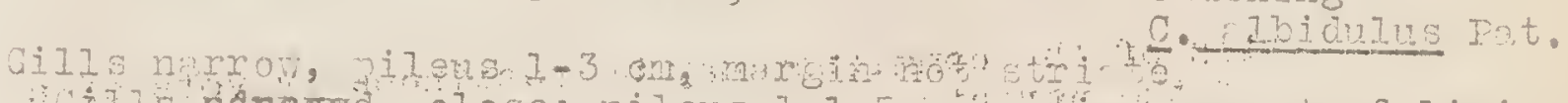

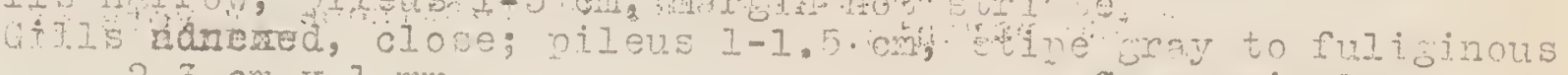

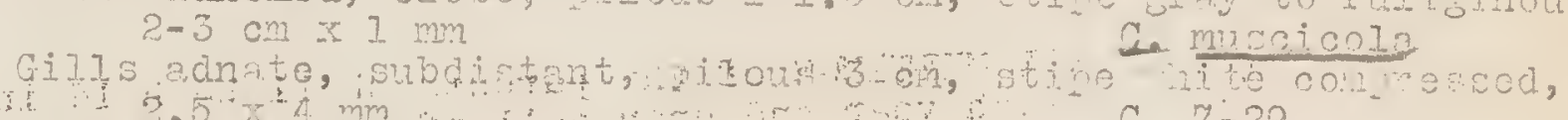

Pilms it, $4 \mathrm{~cm}$, jitis nariow, close,

C. $7-29$

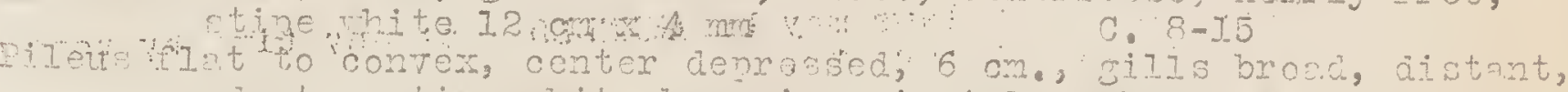

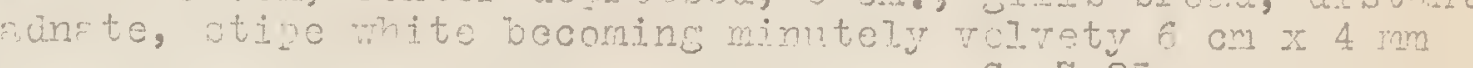

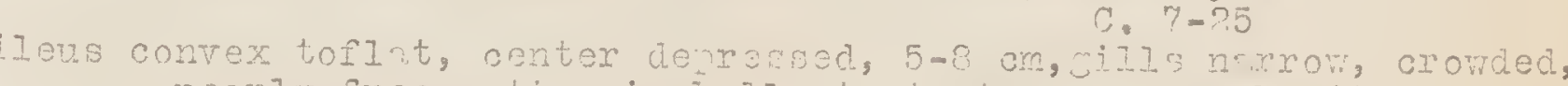
nerly rree, stipe isabelfa tc sestroeous rouch ith achles nointinc notard, $3 \mathrm{~cm} x 3-4 \mathrm{~mm}$ c. Jonryana

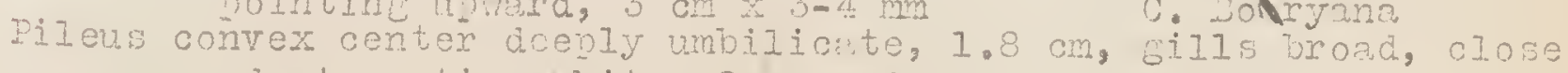
adnate, stipe hite, 2 cm $x 2 \mathrm{~mm}$. $9.7-30$

lexs some shad of ourple, blue ox green

Tileus doen azure (53), glabrous, fills brond, desn azire blue

ile cyanocenhalus

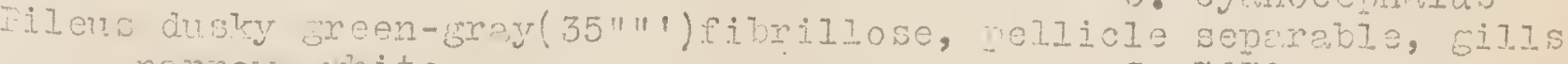
nexpor, wite c. 7670

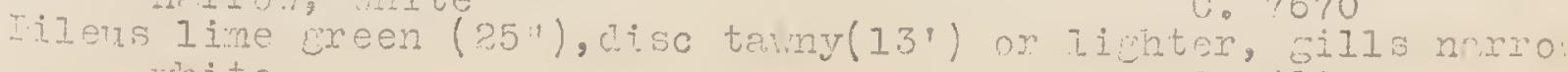

vite C. 4.983

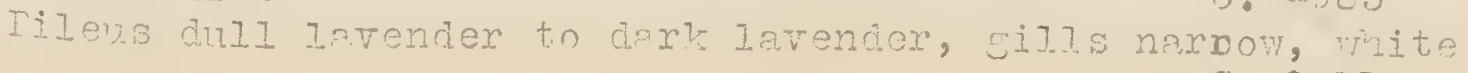

s. $9-17$ 
9. The School shall be administered by an ADMINISTRATIVE COMMT? T⿰氵工丁 of three sets of parents elected by a general meeting of all parents in January of each year to serve for one year.

The committee shall divide its work omong its members as it sees fit.

Withdrawal by members of the committee of their child from the school automatically acts as their resignation from the committee.

In case of resignation from the committee, the remaining members shall elect successor members to serve until the next general meeting of parents.

The comnittee shall follow directions given by vote of the parents at any general meeting.

Action of the committee may be rescinded by vote of a general meeting of the parents.

10. GENERAL MEITINGS of the parents shall be held in september and January of each year and upon call of the committee.

11. These by-laws may be AMENDED et any time by vote of a general meeting of parents. 
Collybia

of Higrophono (Enttygn)

Pileus viscid, white with a few brown patches of universal veil, $3 \mathrm{~cm}$, white;maryin not striate, flesh very thin, almost membranous; gills broad, white, adnate, subdistant;stipe slender,cartilaginous, $3 \mathrm{~cm} \# 2 \mathrm{~mm}$, tapering downward, smooth white; spores white

Specimens examined:

Costa Rj.ca, Limon, Rose Hill above Rio Pacuare, Alt, 100m, Catt, Dgdye, 


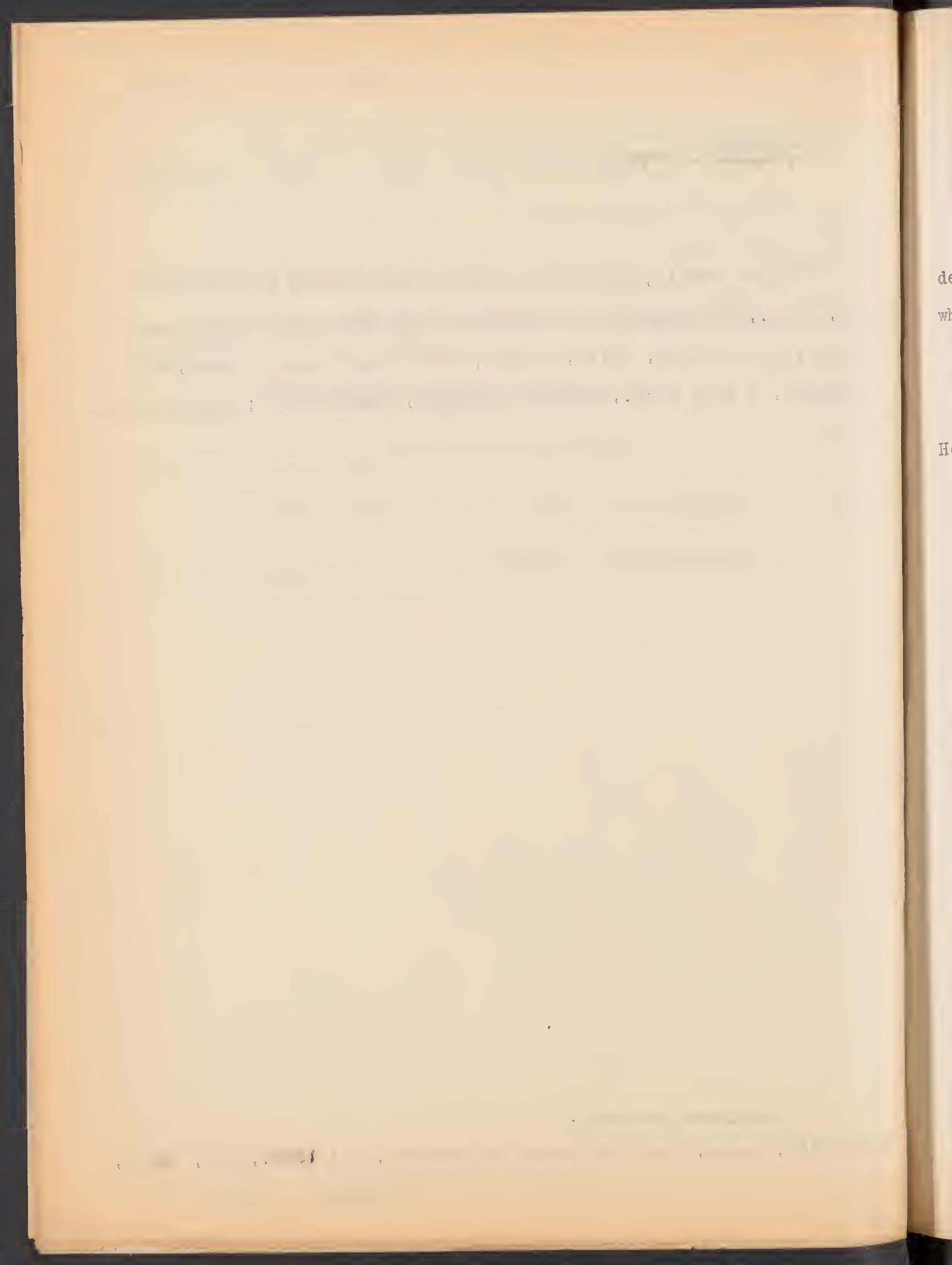


Collybia.

Pileus hemispheric, $1 \mathrm{~cm}$, viscid, umbilicate with tiny umbo in depression, margin inrolled, old gold; gills crowded narrow adnate white or concolor; stipe tiscid, $5 \mathrm{~cm} \times 2 \mathrm{~mm}$ cartilaginous concolor 


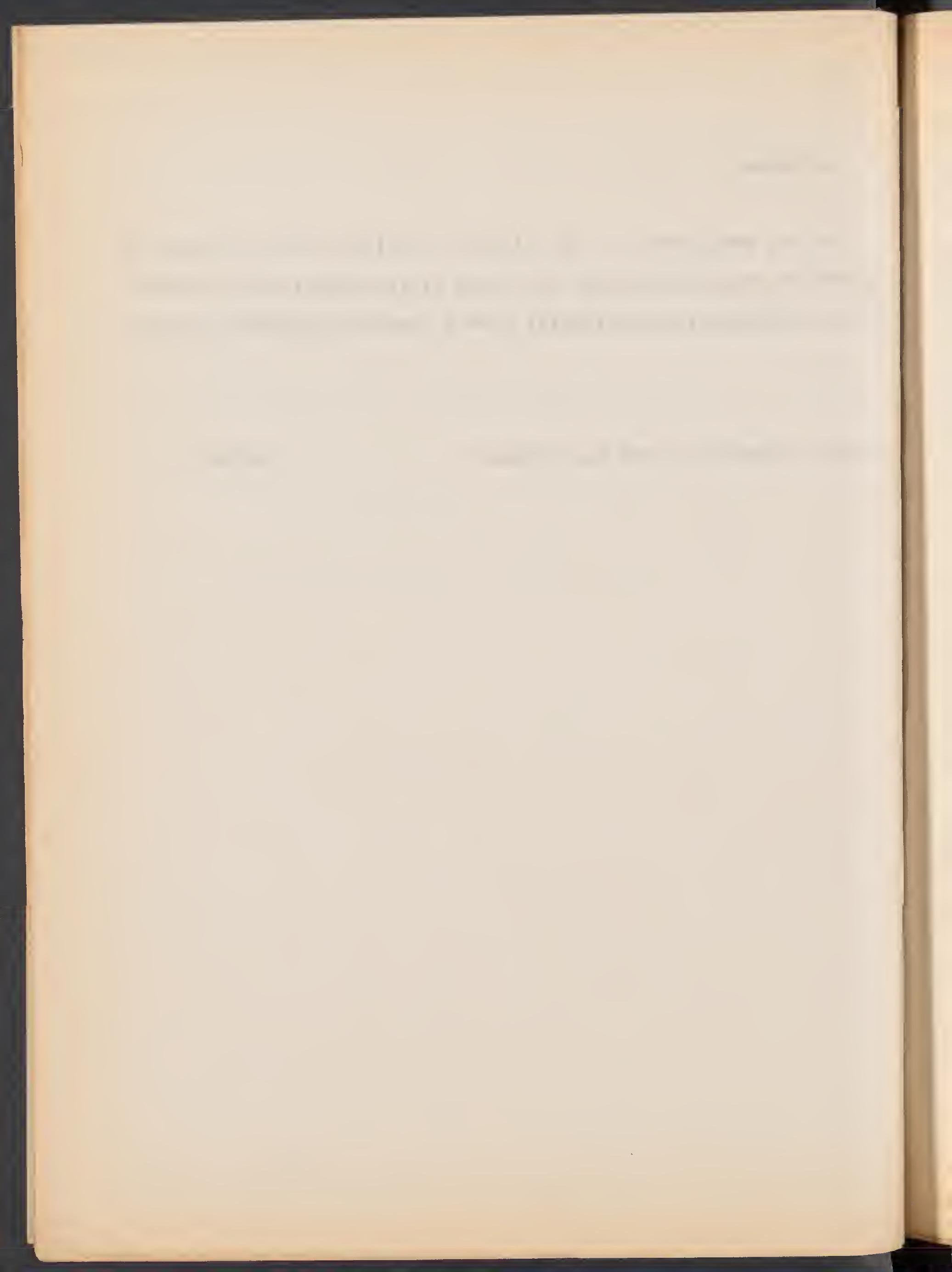


Collybia

Pileus viscid, expanded, slichtly depressed, $6 \mathrm{~cm}$, marein very slightly, coarsely striate, surface with aresden brown areolate remains of universal veil, cartridee buff shading to white maryins; flesh watery; fills thick, subdistant, broad, white, sinuate adnate; stipe cartilakinous $3.5 \times 4 \mathrm{~mm}$, tough, equal ending in bulb $9 \mathrm{~mm}$ diam. silky shining.

On dead wood

Slecimens exarnined:

Costa Rica: Cartaco, Laeuna de Bonilla Dodee, Catt \& Thomas 5868 


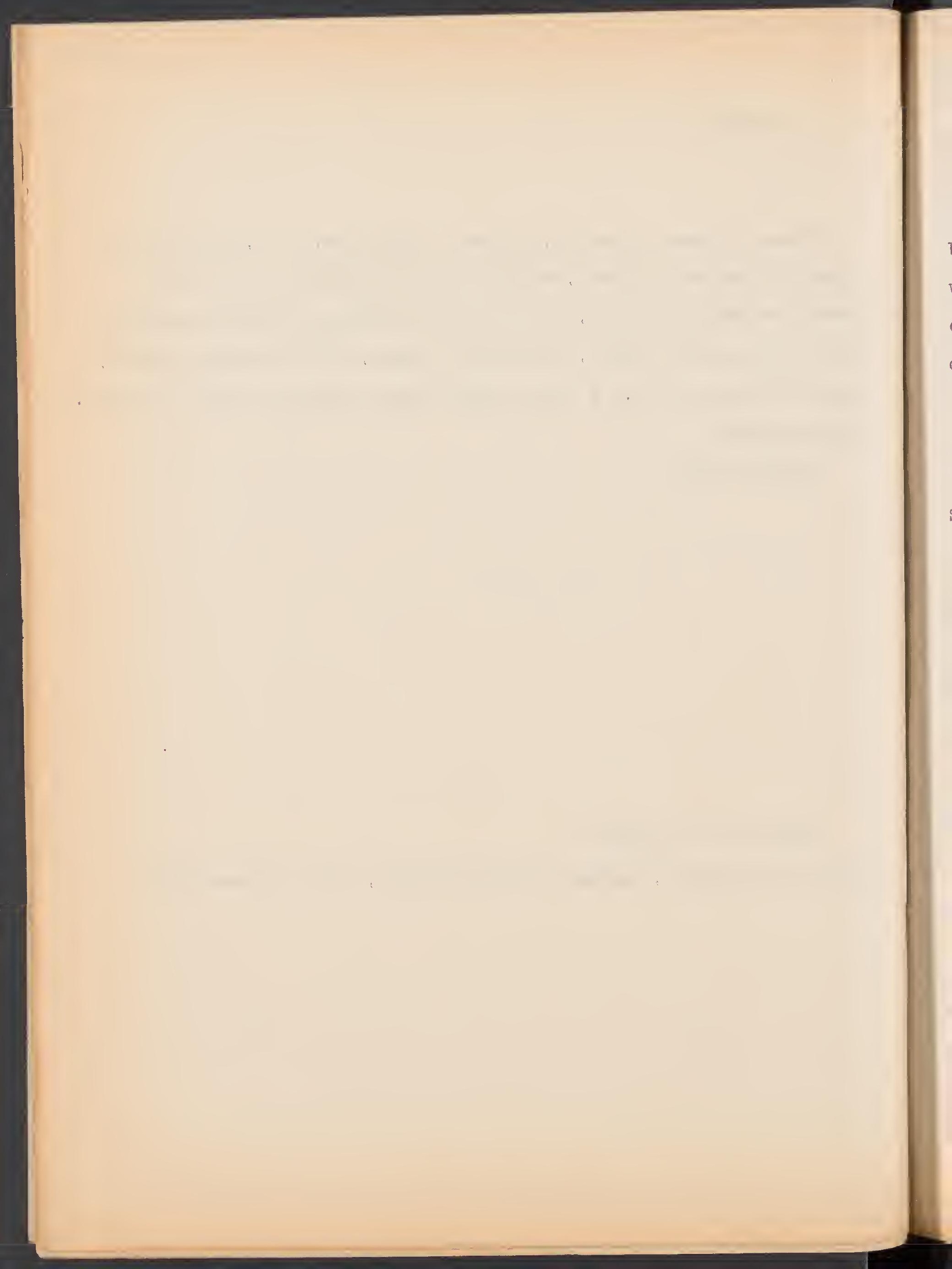


Collybia

(Pleurotus)

Pileus $5 \mathrm{~cm}$, margin narrowly striate, smooth, white to vinaceous buff, dotted more or less concentrically with tufts of universal veil which are avellaneous in color; gills ventricose sinuate adnate with decurr ent tooth, white subdistant; stipe central appearing lateral from position of growth, fleshy, $3 \mathrm{~cm} \times 3 \mathrm{~mm}$, white pruinose furfuraceous, base bulbous

Rotten branch on recently felled tree

SanJosé, R. Paquita, $2 \mathrm{~m}$., Dodge \& Goerger $8-14$ 


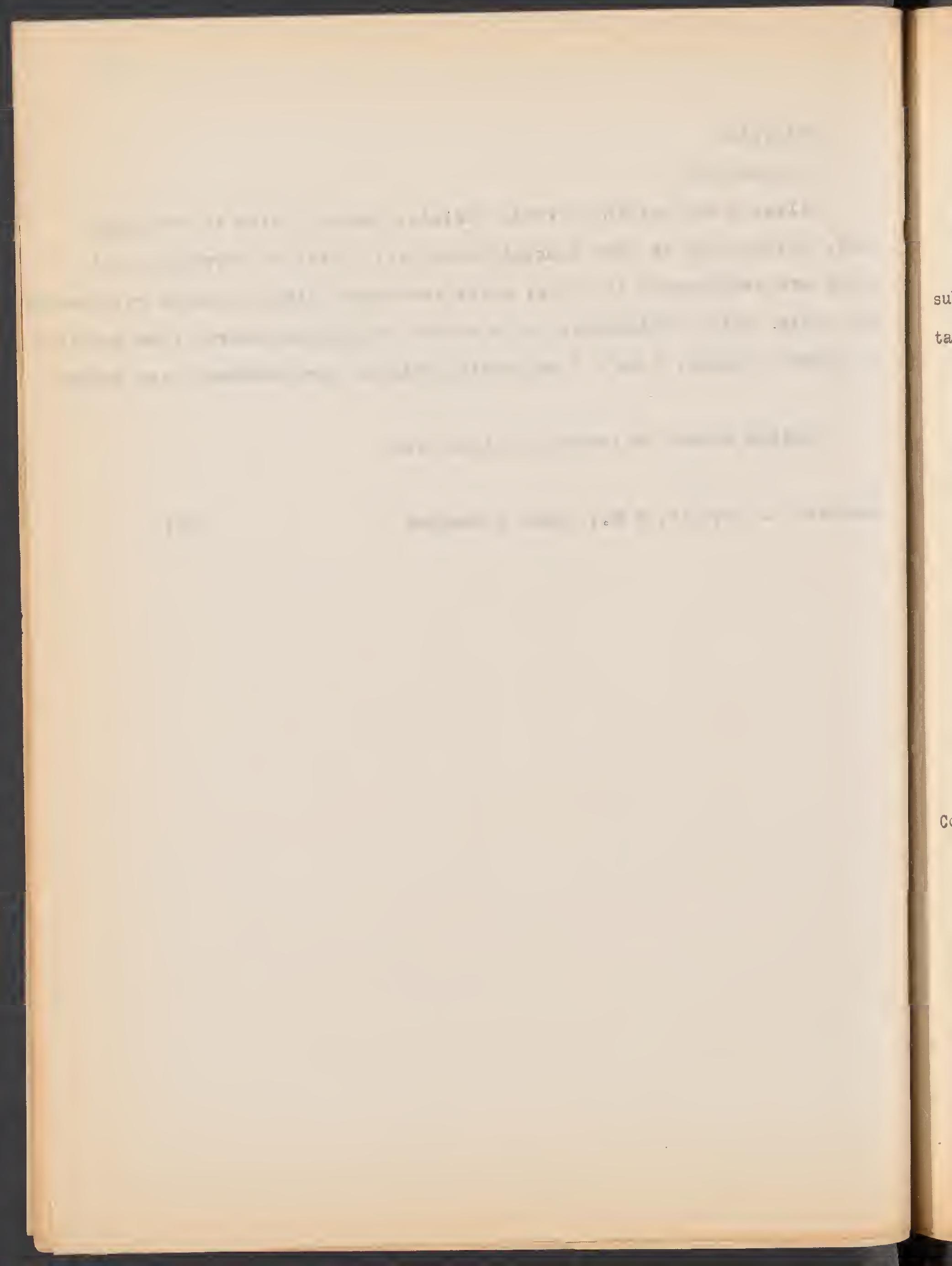




\section{collybia}

pileus viscid, pure white, marein slightly striate, $4 \mathrm{~cm}$; Eills subdistant, broad, white, adnate; stipe somewhat fl shy, cartilaginous taperine downward $8 \times 4-3 \mathrm{~mm}$, white.
Sama bocality Qung 27 hasplocerse a

Costa Rica: Puntarenas, Osa, Rufstero Sandalo 5-20 m, Dodee 7716 Dodget Goenger ang 27. 


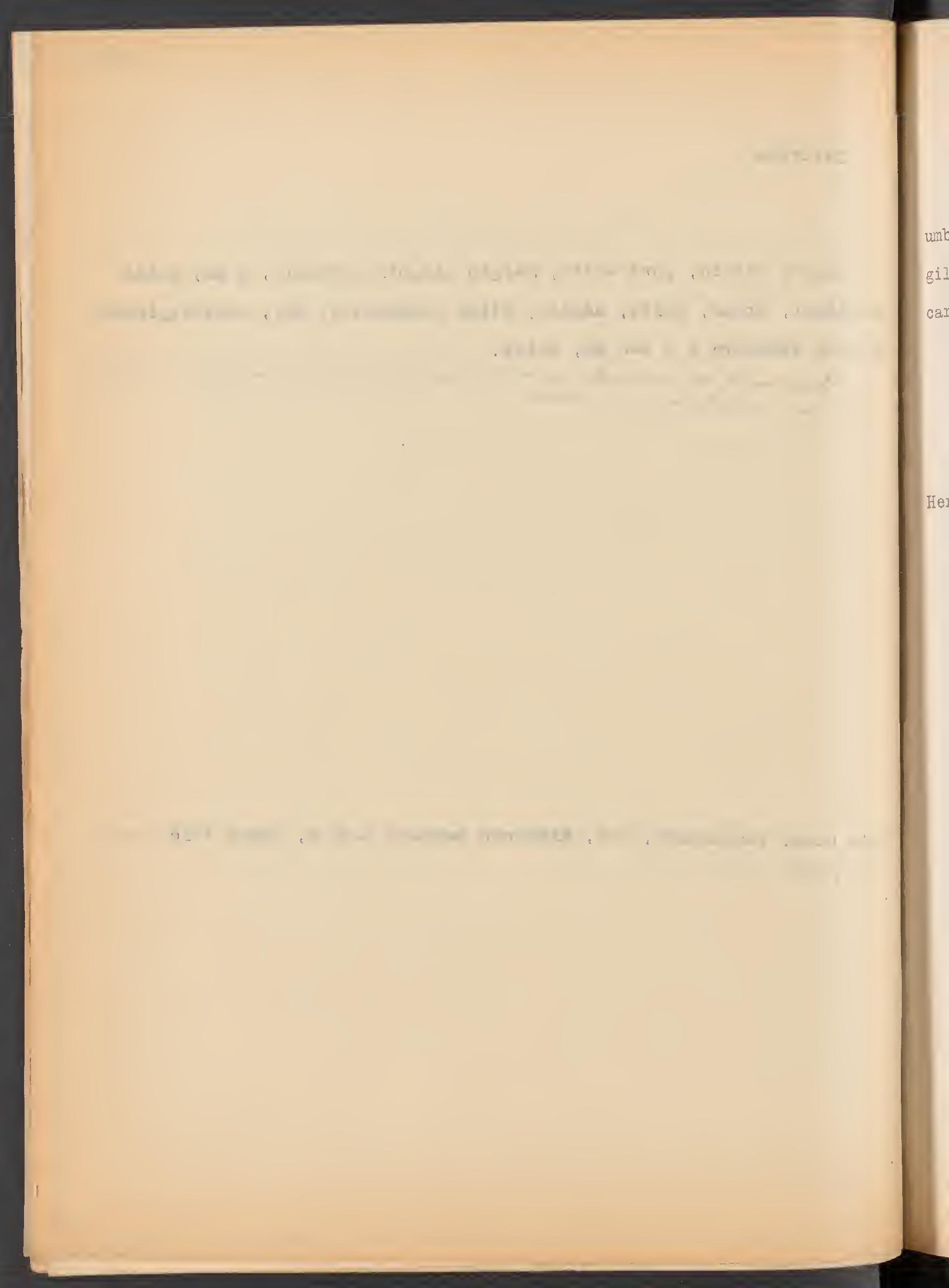


Collybia

Pileus hemispheric, viscid, sulcate striate over gills, umbonate, 17 . 19

unbo Dresden brown, light cadmium, striate with dresden brown, $2 \mathrm{~cm}$, gills adnate, light cadmium, subdistant, broad; stipe $11 \mathrm{~cm} \times 3 \mathrm{rm}$, cartilaginous, viscid, light cadmium, darker below,

Very cespitose

Heredia, Chahuites, 1600 m., Goerger

$9-11-36$ 

Collybia

Pileus expanaea, striate, $4 \mathrm{~cm}$., Saccardo's umber, moist, subviscia; gills, white, crowuea, anate, nearly Iree, ventricose; stipe shining, tapering downwara; pale chalcedonyyellow, cartilaginous, $3.5 \mathrm{~cm}$. Long, $5 \mathrm{~mm}$. above, $3 \mathrm{~mm}$. below, fibrillose.

Mossy 108 in potrero, south of house.

Specimens examined:

Costa Rica, San Jose, Rancho Reaondo, A It. 2160-2180 m.

Döage \& Thomas, 5062 a 


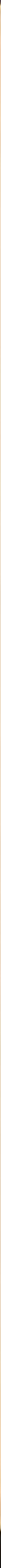


Coliyuia

Pileus up to $9 \mathrm{~cm}$, watery, viscid, between wood brown and buffy brown, lighter on coarsely striate marein; gills broad, white, sinuate adnate, subdistant; stipe cartilatinous stuffed, white $4.5 \mathrm{~cm} \times 5-7 \mathrm{~mm}$ surface dull wxa equal with bulbous base $13 \mathrm{~mm}$ somewhat compressed. cespitose on dead wood

\section{Specimens examjined}

Costa Rica:Cartago, Jaeuna de Bonilla, Dodee, Catt \& Thomas 58629 


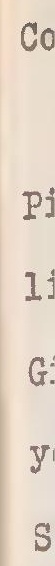


Collybia velutipes group

Pileus expanded, viscid, 3cm., margin striate, chestnut, slightly lighter at margin

Gills sinuate, adnate, ventricose, close, Naples yellow stained yellow ochre on edges

Stipe $2 \mathrm{~cm}$. $x 3 \mathrm{~mm}$. , dense, velvety tomentose, mummy brown or darker

Solitary on decaying log

Costa Rica, San José-prov., Rancho Redondo, 2150m.

Dodge \& Goerger 
Collybie velutipes group

Pileus 4 cr., nearly flat, margin striate, sulcate, surface viscid, disc Sanford brown, rest raw sienna; gills with small cross gills, adnate subdistant, mdeium width, baryta yellow; stipe velvety, 6 cr x $2.5 \mathrm{~mm}$, tough, cartilaginous xanthine grange to Argus brown

San José, Paquita Hill, $15 \mathrm{~m}$, Dodge \& Goerger $8-15$ 


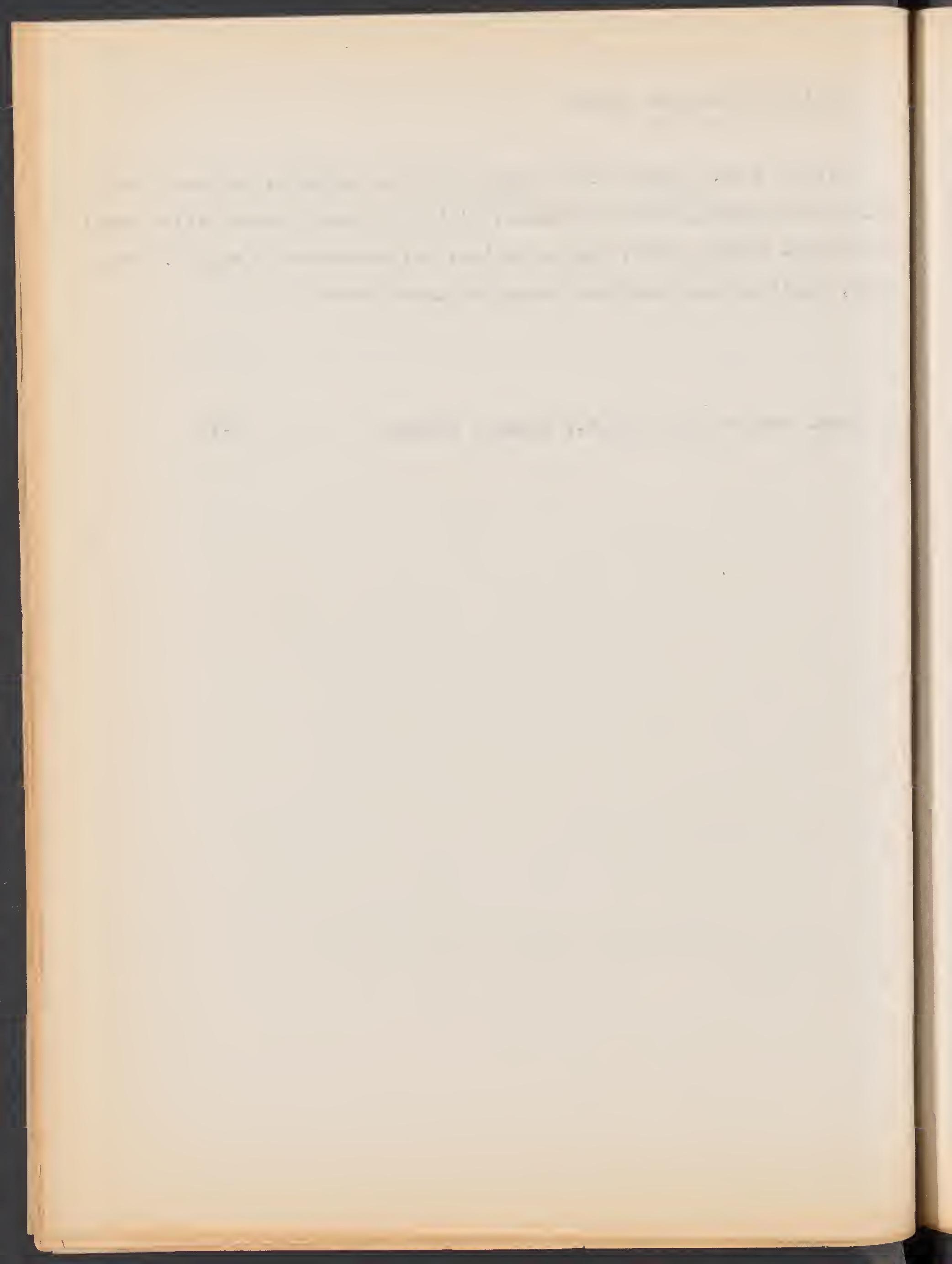


Collybia velutipes group

Pilei up to $2.3 \mathrm{~cm}$, $\nabla$ iscid, disc ochraceous orange shading irregularly toNaples yellow, margin thin, smooth; gills ventricose, adnexed, subdistant, white; stipe cartilaginous fragile, velvety, cream above shading to mummy brown below, $2 \mathrm{~cm} \times 2 \mathrm{~mm}$,

Cespitose on decaying Erythrina post in front yard

San José, Guayabillos $2250 \mathrm{~m}$., Dodge \& Goerger $7-16-36$ 
Collybia velutipes'.

P1leus convex, $2 \mathrm{~cm}$., vigcid, amber-brown, shading to $17 \%$ antimony-yellow, margin; tlesh thin,yellowish;gills adnate, closeventricose; stipe cartilaginous, slender, velvety, Mars 3 . brown, shading to yellow above, $13 \mathrm{~mm}$. long $2.5 \mathrm{~mm}$. diamter, caespitose.

\section{Specimens examined:}

Costa Rica, San Jose, Rancho Redondo, Alt. 2300m. Dodge \& Thomas 5308 


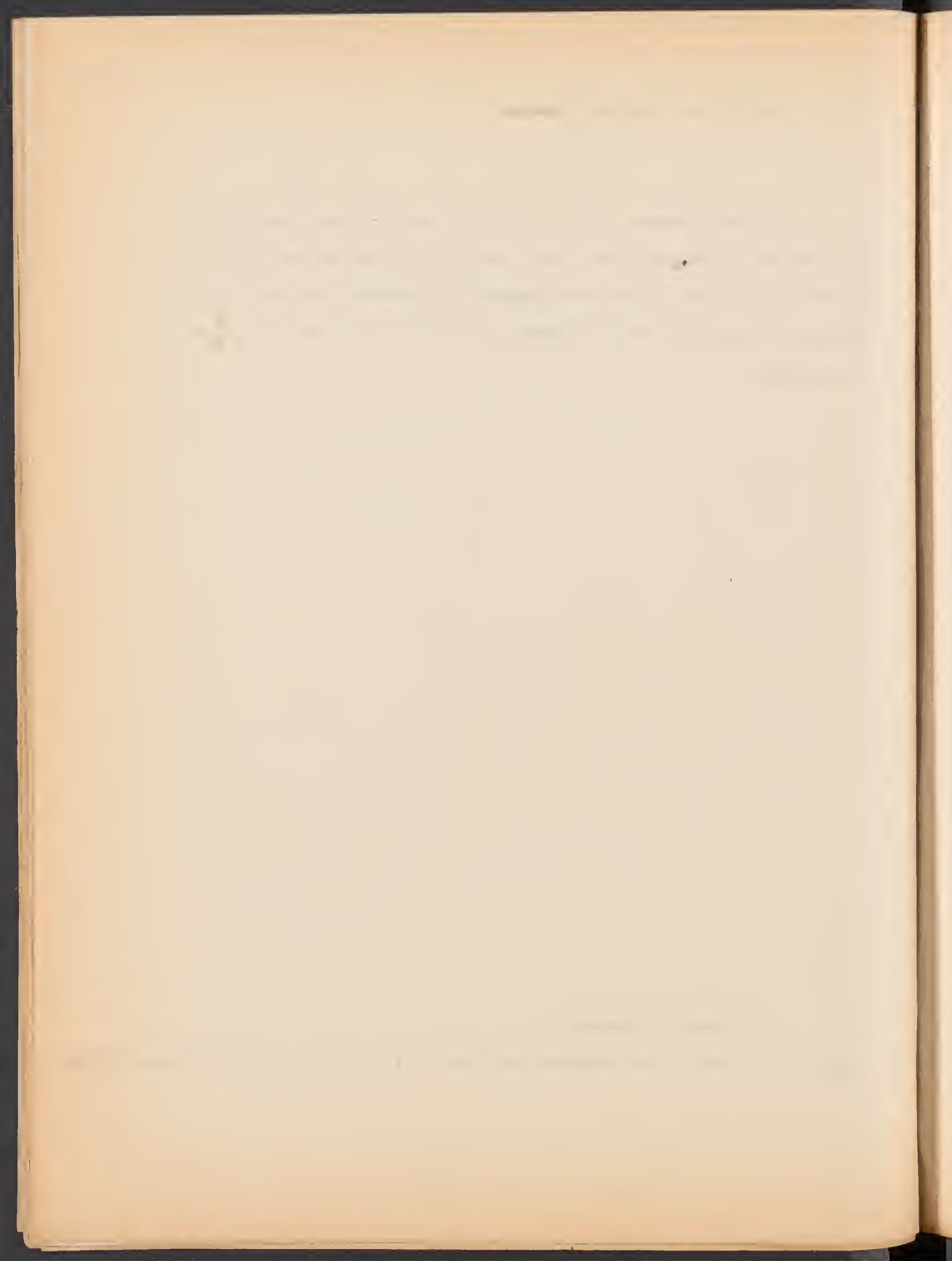


Collybia velutipes group

Stipe caespitose, viscid with velvety tomentum.

Dead wood in potrero $S$. of House.

Specimens examined:

Costa Rica, San Jose, Rancho Redondo, Alt 2160-2180. Dodge \& Thomas 5309 

Collybia velutipes group

Plieus viscid, Iighter color; stipe mucn longer, less densely cespitose, less densely velvety.

Dead wood in potrero s.of tousa

\section{Specimens examined:}

Costa Rica, San Jose, Rancho Redondo, Alt. 2160-2180 Dodges Thomas, 5310 

Collybia velutipes group.

pileus viscid; stipe velvety; caespitose.

On dead wood in potrero $S$. of house.

\section{Specimens examined:}

Costa. Rica, San Jose, Rancho Redondo, ALt. 2160-2180m. Dodge \& Thomas $53 / 1$ 


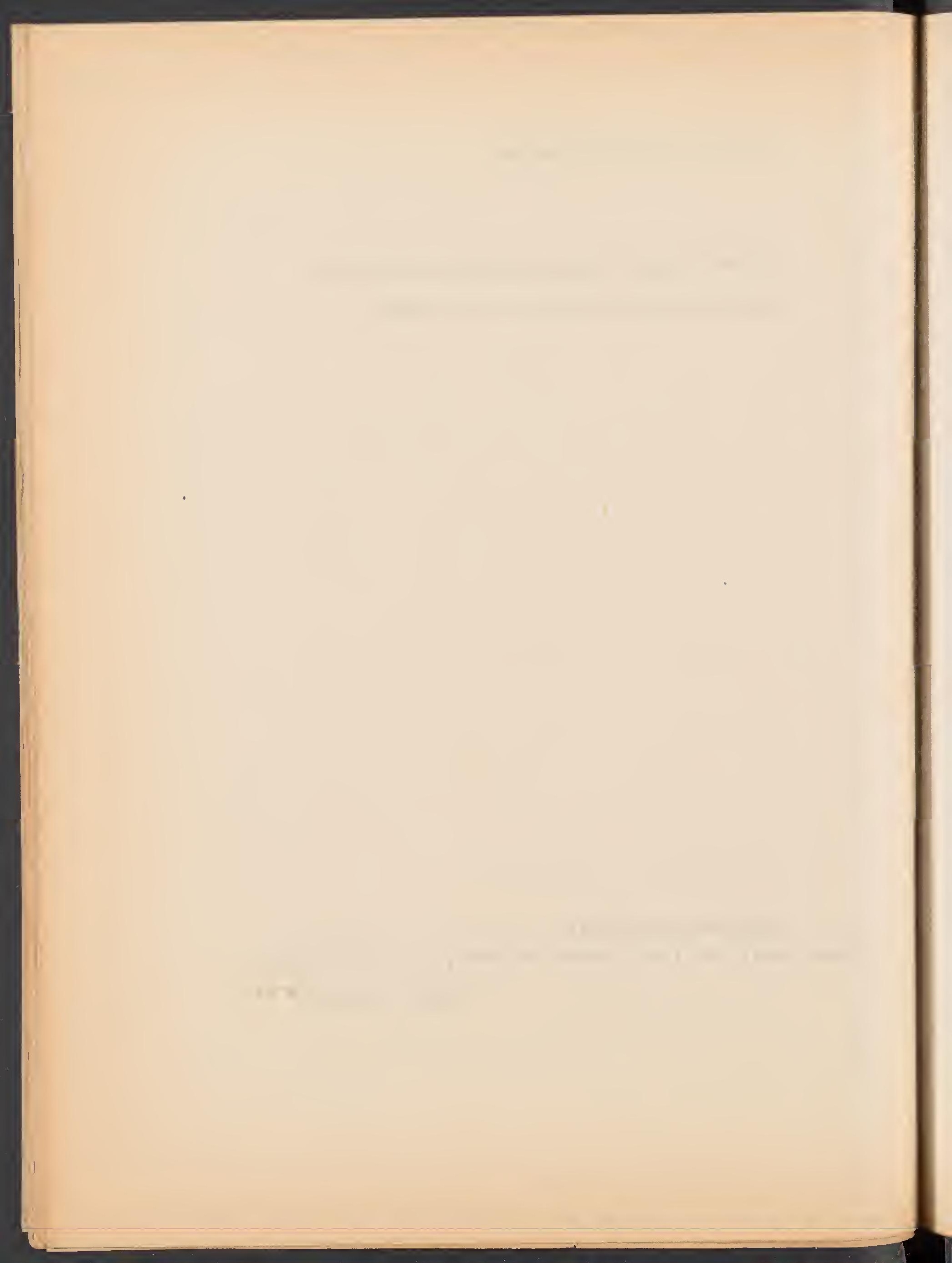


Collybia

Pileus fla.t, $5.5 \mathrm{~cm}$, moist, almost glutinous, shining perhaps slightly fibrillose when dry, striate but not sulcate over gills, pale olive buff to white; gills adnate subdistant, ventricose, white; stipe $3 \mathrm{~cm} \times 4 \mathrm{~mm}$, cartilaginous, quite tough, silky, compressed.

In soil on creek on tram east of Guacimo bridge

Limon, Siquirres, Castilla, $20 \mathrm{~m}$. F. Nevermann 

Col1ybia.

Pileus mars brown before laceration, conic expanded with prominent conic umbo, surface lacerate fibrillose, margin lacerate, $2.5 \mathrm{~cm}, 1 \mathrm{~cm}$ tall, gills adnate, white close,broad, stipe white $5 \mathrm{~cm} \times 5 \mathrm{~mm}$, tapering upward, very fragile

On soil in forest along quebrada

Sna José, Guayabillos, 2300 m., Dodge \& Goerger 

Collybia

$$
\text { tenuipes group }
$$

Pileus hemispheric, Icm., densely scaly with pyramidal tufts of hairs, Prout's brown

Gills adnate, white, subdistant, broad

Stipe tomentose, floccose, whitish above more or less concolorous or lighter, $2 \mathrm{~cm}$. $\times 1.5 \mathrm{~mm}$.

Costa Rica, Sam Jose prov., Guayabillos, potrero 2200m. 


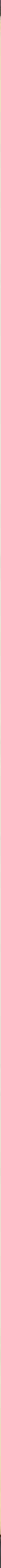


Collybia

Pileus $2.8 \mathrm{~cm}$, flat or very broadly umbonate; disc pyramidal, scaly, onionskin pink; lighter toward margin due to growth pulling the colored hair tufts further apart

Gills close, broad, sinuate, adnexed to nearly free white stipe, light ochraceous salmon, $3 \mathrm{~cm}$. $x 3 \mathrm{~mm}$, silky

Costa Rica, Limón prov., Castilla farm, $20 \mathrm{~m}$. $7-25-36$

Dodge \& Goerger 

Collybia

Pileus hemispheric, 3 cm, hygrophanous, disc decorated with pyramidal tuBts of hairs, margin striate smooth, disc light salmon, margin russet; gills subdistant, adnate, broad, white; stipe floccose light ochraceous salmon, becoming russet when handled, $3.5 \mathrm{~cm} \times 5-7 \mathrm{~mm}$, tapering upward

SanJosé, Guayabillos, $2200 \mathrm{~m}$., Dodge \& Goerger $7-11-36$ 


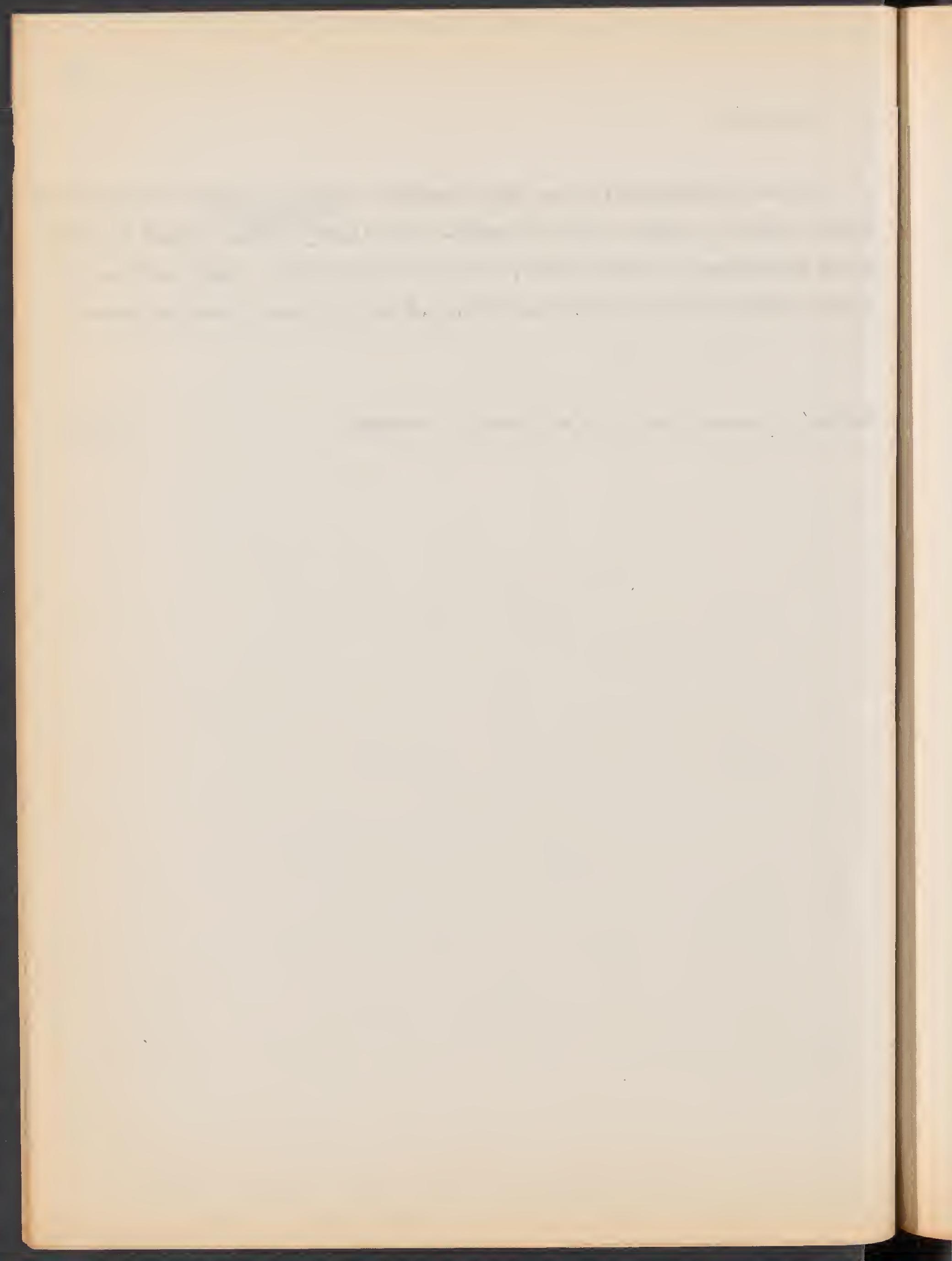


Collybla near C. subflavescens. Mumr.

Pileus campanulate, minutely scaly, cuticle separable, flesh $2 \mathrm{~mm}$. On disc, thin at margin; gills narrow, crowded, nearly tree; stipe slender, cartillaginous, sturted, silky above, furfuraceous-scaly below, cinnamon-buff with Saccardo umber sclaes.

Specimens examined:

Costa Rica, Cartago, Pejivalle, Alt. 650-820, Dodge \& Thomas Azt $=4471$ 


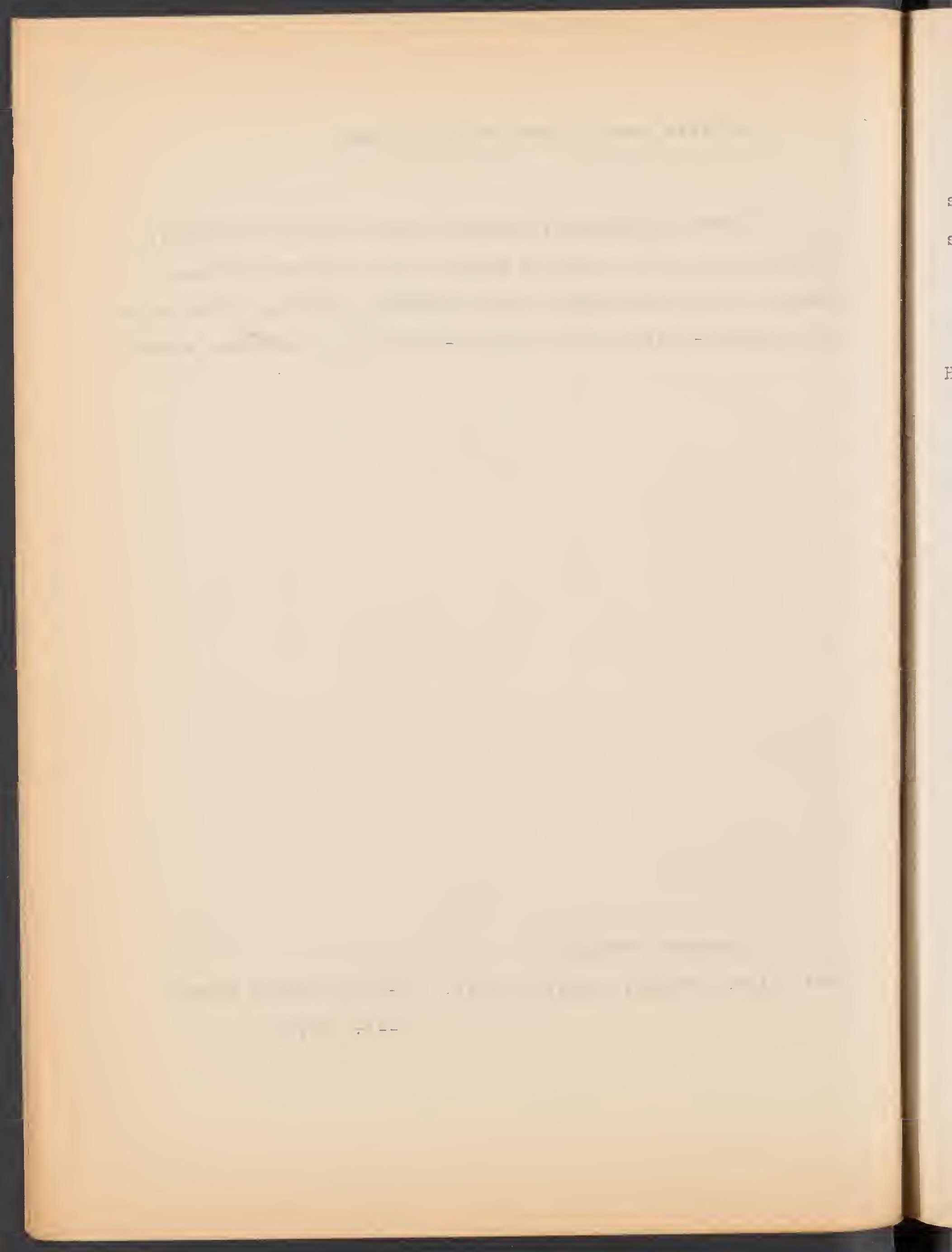


Collybia

Pileus white, hemispheric, $1 \mathrm{~cm}$ disc slightly depressed, and scaly, rest fibrillose spongy; gillg broad, adnate, white, close, stipe $4 \mathrm{~cm} \times 2 \mathrm{~mm}$, white cartilaginous, hollow

Heredia: Chahuites, 1600 m., Goerger $9-11-36$ 


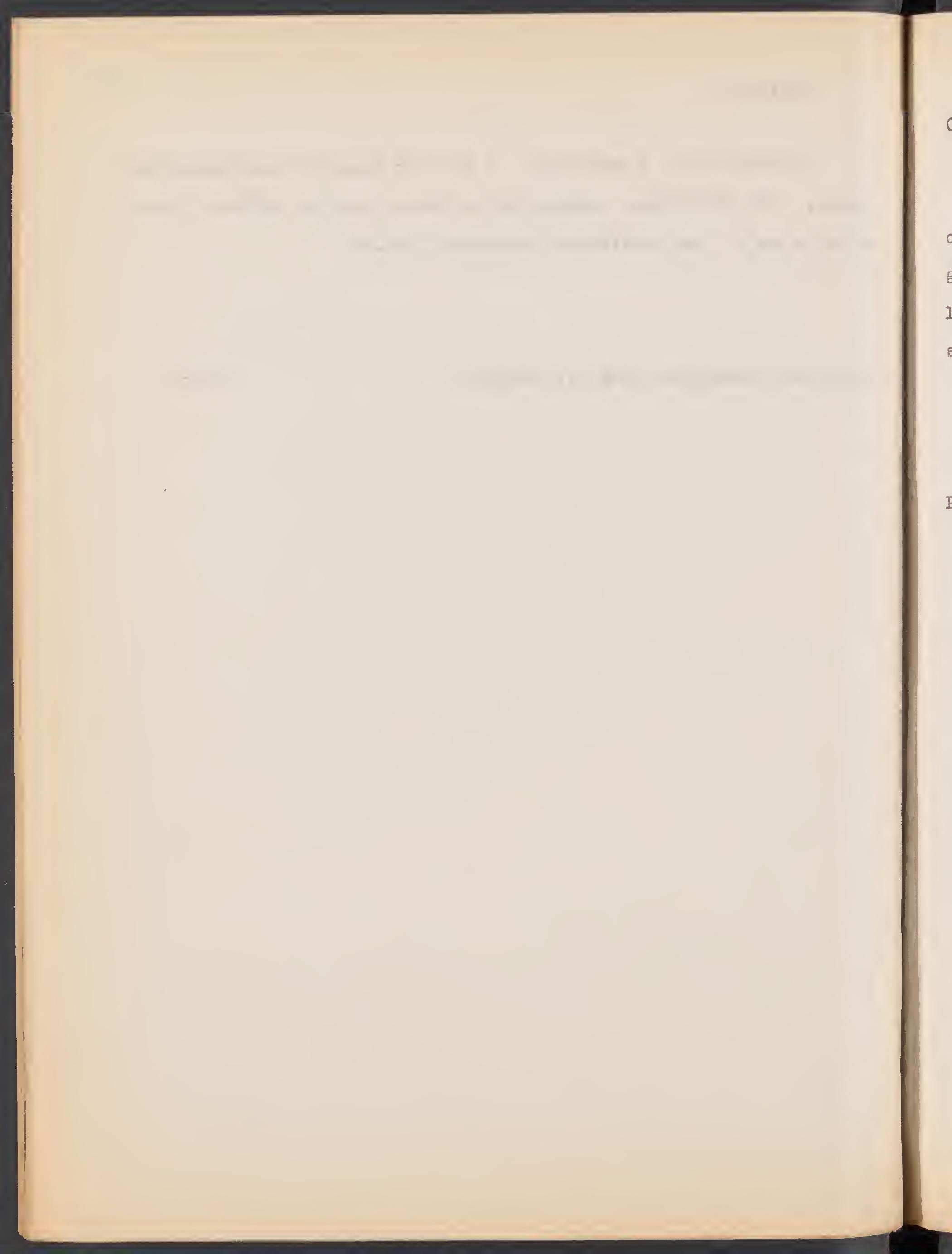


Collybia A

Pileus up to $4 \mathrm{~cm}$ in diameter convex to irregularly expanded, sulcate over gills, surface tomentose scaly, buckthorn brown a warm buff background, margin irregular; gills very distant and broad, white more or less watersoaked, adnate; stipe short, $2 \mathrm{~cm} \times 2.5 \mathrm{~mm}$, hygrophanous white soon blackening, cartilaginous

On decaying stick

Puntarenas, Osa, R. Sándaio, $2 \mathrm{~m}$. Dodge\& Goerger $8-21$ 

Colzybia

Pileus tomentose, buffy brown quickly blackening, conrex up to $3 \mathrm{~cm}$ diam; eills adnexed, close white, soon blackenins in spots narrow; stipe flattened, subterete below tomentose pruinose, black $5 \mathrm{~cm} \times 5 \mathrm{~mm}$

Speciratis examined:

Costa Rica: San Jose, Rancho Redondo Dodge \& Thomas 5368 


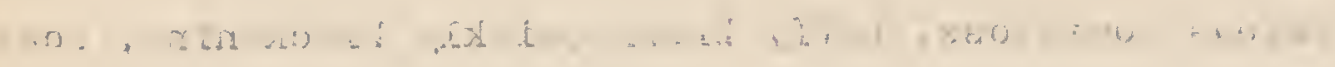


Collybia

Pileus $3 \mathrm{~cm}$, flat strigose velvety disc mars brown to mumby brown to buckthorn brown, margin very slightly coarsely striate; gills distant, broad white more or less merulioid between, adnate; stipe cartilaginous tough, buckthorm brown, silky, $4 \mathrm{crn} \times 2 \mathrm{~mm}$.

Puntarenas, Osa, R. Sandalo, $2 \mathrm{~m}$. Dodge \& Goerger 


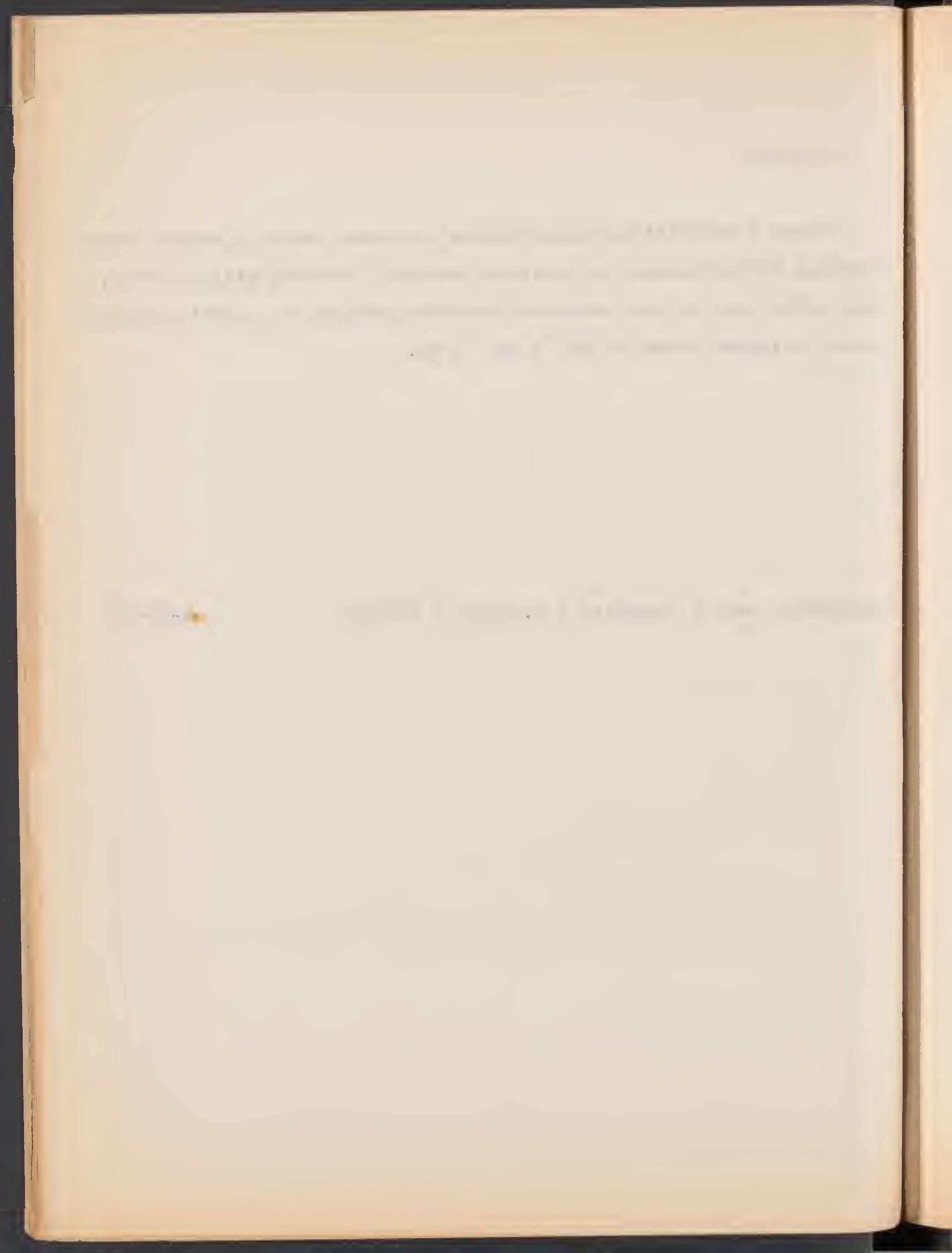


Collybia nearest C. setulosus (see painting 4472)

Lamellae narrow, crowded, nearly free, prominently umbonate, gibbous.

Specimens examinea:

Costa Rica, Cartago, Pejivalle, Alt.650-800m. Dodge \& Thomas 4472 


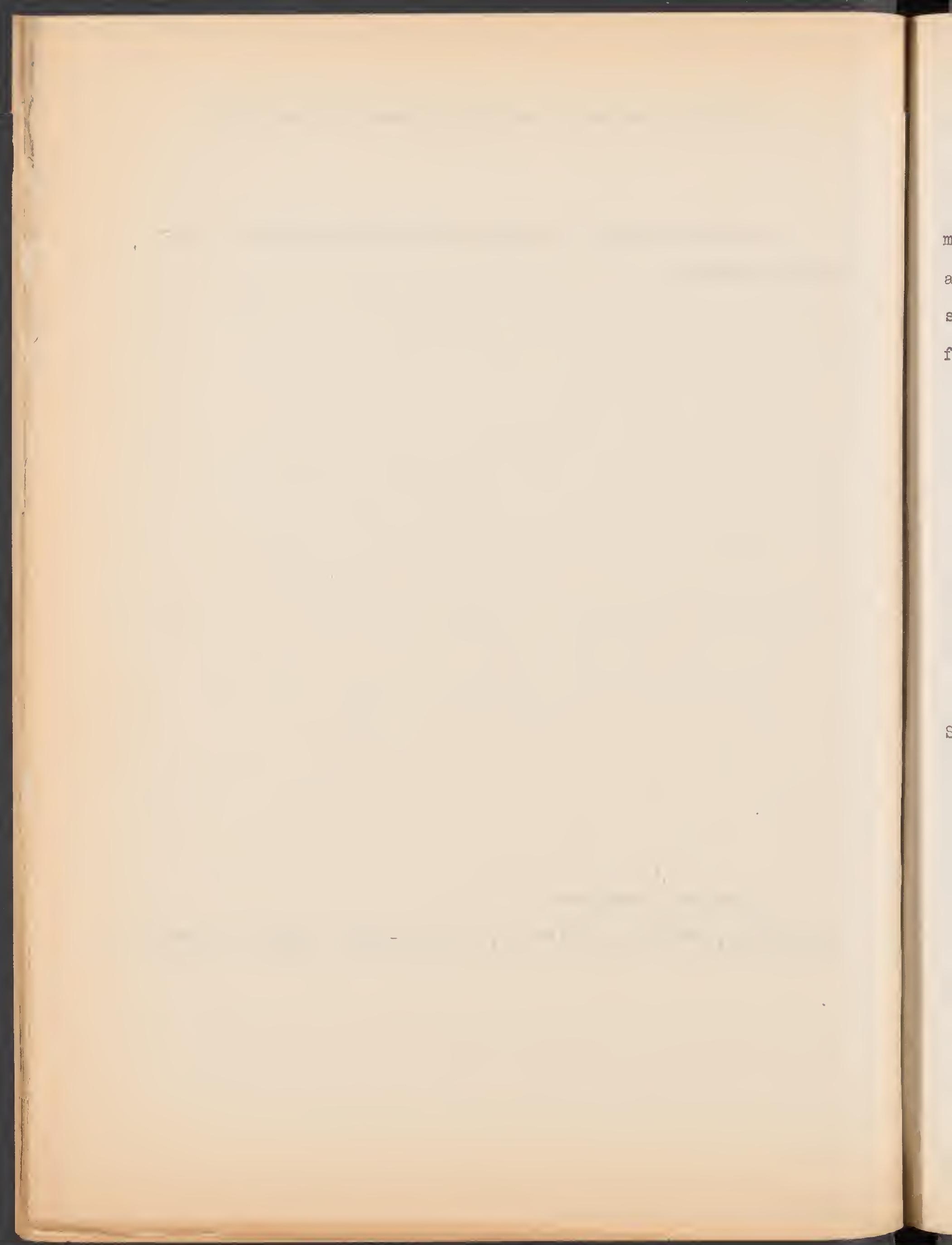


Collybia

Pileus $2-3 \mathrm{~cm}$, convex to haerly flat, surface tomentose, short margin even, disc buckthorn brown shadingthrough yellow ochre to antimony yellow, subhygrophanous; gills growded, narrow, anitmony yellow stipe yellow ochre, cartilaginous tough, slightly velvety above, very faintly fibrillose, $2 \mathrm{~cm} \times 2 \mathrm{~mm}$

On very rotten log

San José, R. Paquita, 3-5 m., Dodge \& Goerger 

Collybia

Pileus $1.5 \mathrm{~cm} .$, spongy, tomentose; disc ochraceous orange; rest Naples yellow,

Gills adnate, broad, subdistant, Naples yellow

Stipe $3.5 \mathrm{~cm}, \times 1.5 \mathrm{~mm}$, slightly bulbous below and above, Naples yellow above, deeper below

Costa Rica, Puntarenas prov., Osa, R. Sándalo, 1-5m, 

Collybia

Marasmius of Berteroi
haematocephalus

Pileus $3.5 \mathrm{~cm}$. campanulate with prominent, rounded umbo, velvety, tomentose, almost membranous, mahogany-red with umbo bay to chestnut; gilis very broad, adnexed, waxy-white with English red edge; stipe hollow, fragile, white, shading to bay below; stipe $9 \mathrm{~cm}$. \# $3.5 \mathrm{~mm}$.

Under Quercus

Specimens examined:

Costa Rica, San Jose, Rancho Redondo, Alt. 2250m., Dodge \& Thomas 5028 
Collybia?

Pileus hemispheric, margin inrolled, conic umbonate, umbo disappearing in age, hygrophanous, umbo ochraceous orange, rest antimony yellow, very minutely velvety; gills adnate, close, narrow, lemon yellow stipe cartilaginous tough, $2 \mathrm{~cm} \times 1.5 \mathrm{~mm}$ lemon yellow, silky fibrillose

Puntarenas, 0sa, R. Sándalo, $2 \mathrm{~m}$. Dodge \& Goerger 

Collybia

Mycena

Pileus $2.5 \mathrm{~cm} .$, convex, very slifhtly umbonate, slightly velvety, wood-brown to bufy-brown, slichtly darker on the umbo;gills adnexed, thick, white, subdistant; stipe white, slender, cartilavinous, d compressed $2.5 \mathrm{~cm} * 2 \mathrm{~mm}$.

Dead wood.

\section{Specimens examined:}

Costa Rica, Iimon, above Rio Siquirres,Alt. 300m.,Catt, Dodge \&

Thornas 5532




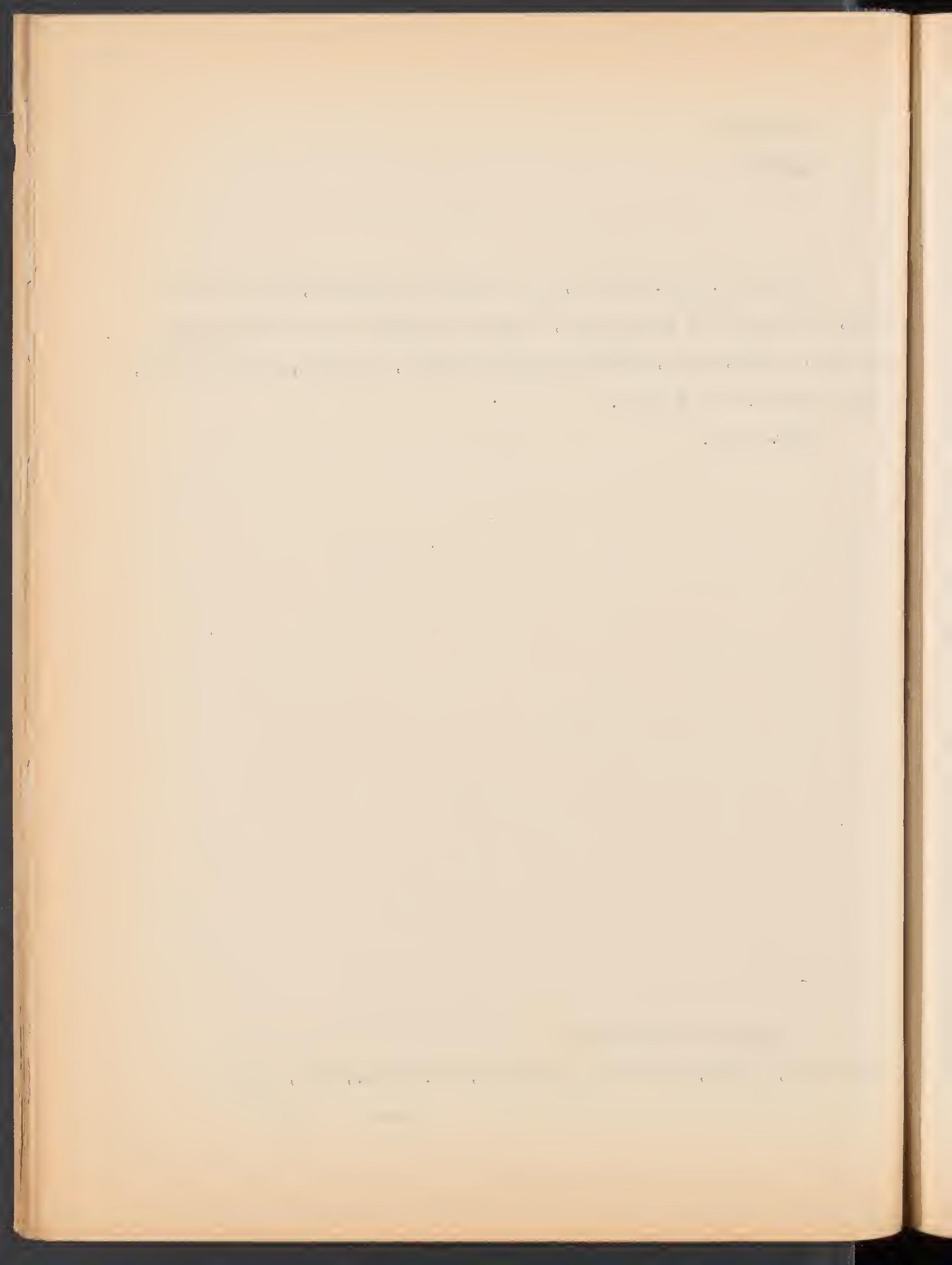


Collybia nean chropopeplus

pileus expanaed, slightly umbonate, antimony yellow to yellow-ocher, velvety to very minutley verrucose, margin crisped, thin; gills white, crowaed, broad anate; stem ochraceousbuff, $3 \mathrm{~cm}$. \# $4 \mathrm{~mm}$.

Potrero

Specimens examined:

Costa Rica, San Jose, Rancho Redondo, Alt. 2550m. Doage \& Thomas 5128 

Collybia

Pileus hemispheric to convex, $2 \mathrm{~cm}$, hygrophanous, minutely velvety disc merulioid, pinkish buff and cinnamon buff; gills white, adnate, sudistant, narrow to mediurn; stipe cartilaginous up to $7 \mathrm{~cm} \times 1.5-1 \mathrm{~mm}$ tapering downward, whitepabove, chestnut or darker below, base with yellowish cottony tomentam, fibrillose

Heredia, Chahuites, $1600 \mathrm{~m}$., Goerger $9-11-36$ 
Collyoia

Pileus convex, $4.8 \mathrm{~cm}$, buckthorn brown, disc wrux darker, very minutely velvety, deeply striate sulcate over gills, almost membranous, gills white, distant, adnate broad; stipe $7.5 \mathrm{~cm} \times 3.5 \mathrm{~mm}$ cartilaginous tough, nearly black

Heredia, Chahuites $1600 \mathrm{~m}$. , Goerger $9-11-36 a$ 


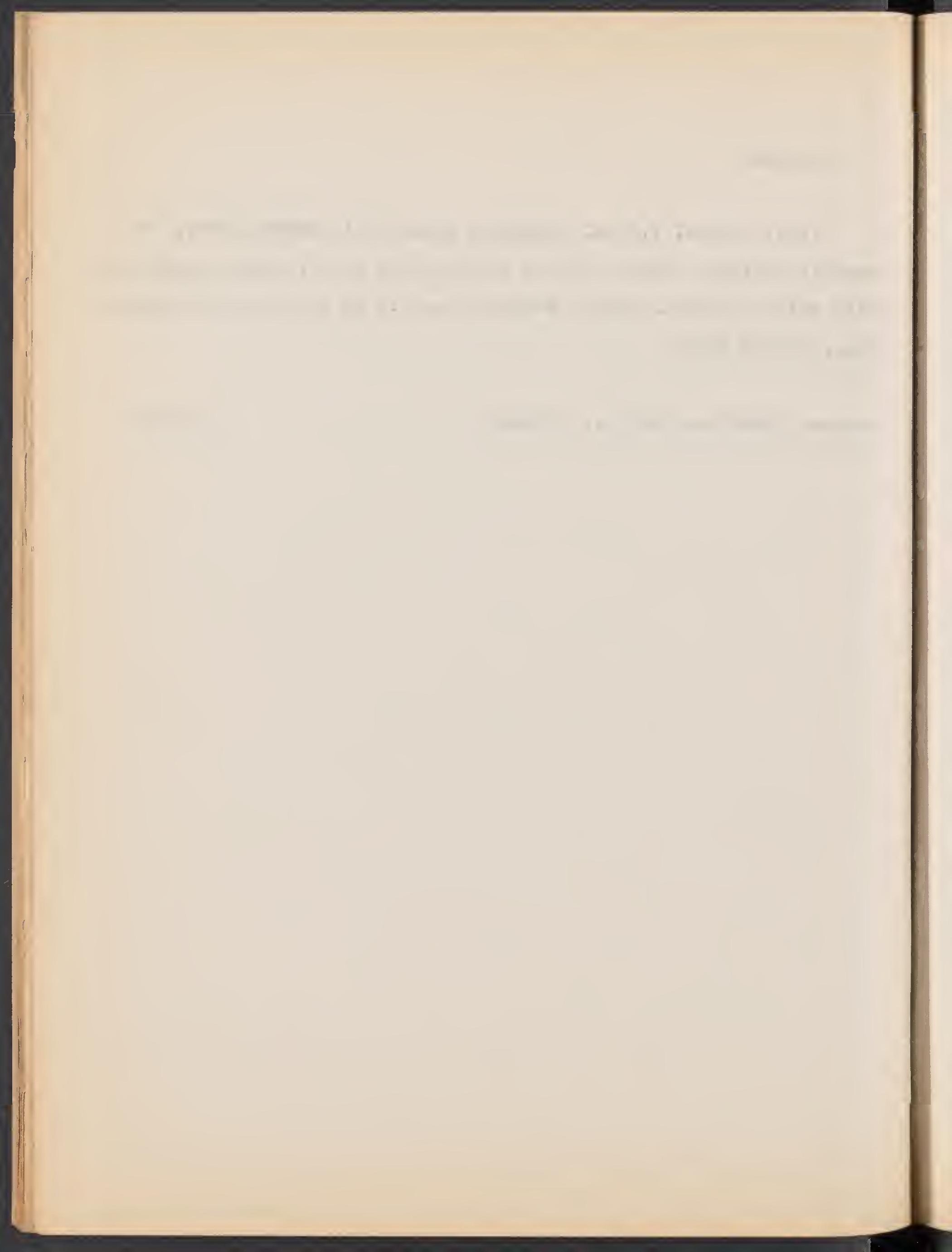


Collybia

Pileus broadly campanulate, velvety, very broadly and shallowly sulcate striate, mars orange to burnt sienna, gills adnexed, practically free, white distant broad; stipe cartilaginous toggh, yellow ochre black

above, shading through chestnut to kekx, silky smooth

Heredia, sto Domingo de Sta Barbara, $1800 \mathrm{~m} .$, Dodge \& Goerger 
Collybia velutipes group

Pileus $4,5 \mathrm{~cm}$., flat or margins inrolled, striate sulcate halfway to disc which is auburn, shading to orange rufous

Gills connected by merulioid cross ridges, sinuate, adnate to nearly free, broad, medium spacing, lemon chrome

-Stipe velvety, cartilaginous, tough, $4 \mathrm{~cm}, \times 3-5 \mathrm{~mm}$, burnt sienna to chestnut

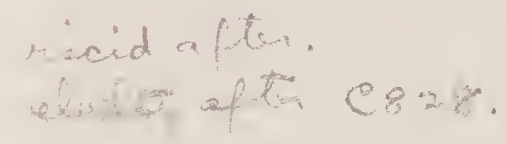

Costa Rica, Puntarenas prov., 0sa, R. Sándalo, 1-5m. 

Collybia

Pileus $2.5 \mathrm{~cm}$. , expanded, deeply umbilicate and striate, minutely tomentose velvety, vinaceous buff, margin crisped; gills broad, sinuate adnate, subdistant, pale cinnamon pink; stipe velvety, $2 \mathrm{~cm} x 2 \mathrm{~mm}$. Prout's brown

Puntarenas, Osa, Q. Huaca, $5 \mathrm{~m} .$, Dodge \& Goerger 



\section{Collybia \& $5 / 28$}

Pileus flat, deeply umbilicat, minutely velvety, disc saccardo umber, rest sepia, margins whitish, not striate, gills close

narrow thick adnate white; stipe cartilaginous, semipellucid, white smooth, $1.5 \mathrm{~cm} \times 2.5 \mathrm{~mm}$ 

Collybia

Omphalina

Pileus up to $3 \mathrm{~cm}$, deeply umbilicate, light brownish olive in depression, margin cream buff to isabelline, very minutezy tomentulose, otherwise smooth, margin not striate although appearing so from the gills underneath in certain lights; gills white, narrow, very close, sinuate decurrent by a tooth; stipe white shading to isabelline $2.5 \mathrm{~cm} \times 4-2 \mathrm{~mm}$ tapering downward

Iimon, Siquirres, Castilla, $20 \mathrm{~m}$., Dodge \& Goerger 
Collybia.

Pileus flat with margins inrolied above, $2 \mathrm{~cm}$. , slightly tomentose, very moist, light browish olive shading to white somewhat irregularly Gills adnexed, very narrow and crovded, white Stipe white, cartilaginous, $5 \mathrm{~cm} . x 2.5 \mathrm{~mm}$, slightly smoky below

very caespitose

On rotten $\log$ in forest

Costa Rica, Heredia prov., Sto, Domingo del Roble, $8-6-36$ Dodge \& Goerger 

collybira

Pileus urto $4 \mathrm{~cm}$, marin inrolled, surface fibrijuose velvety, disc depressed, auburn shdatine to apricot buff; Eills adnate, decurrent by a tooth, close, white; stipe cartilaginous to slikitly fleshy houlow, curiously contorted distorted, 4 m $7.4-5 \mathrm{~mm}$, fihrigloses? silky pure white.

Costa Rica: Puntarenas, Osa, Prerto Jimenez Dodge 7690 


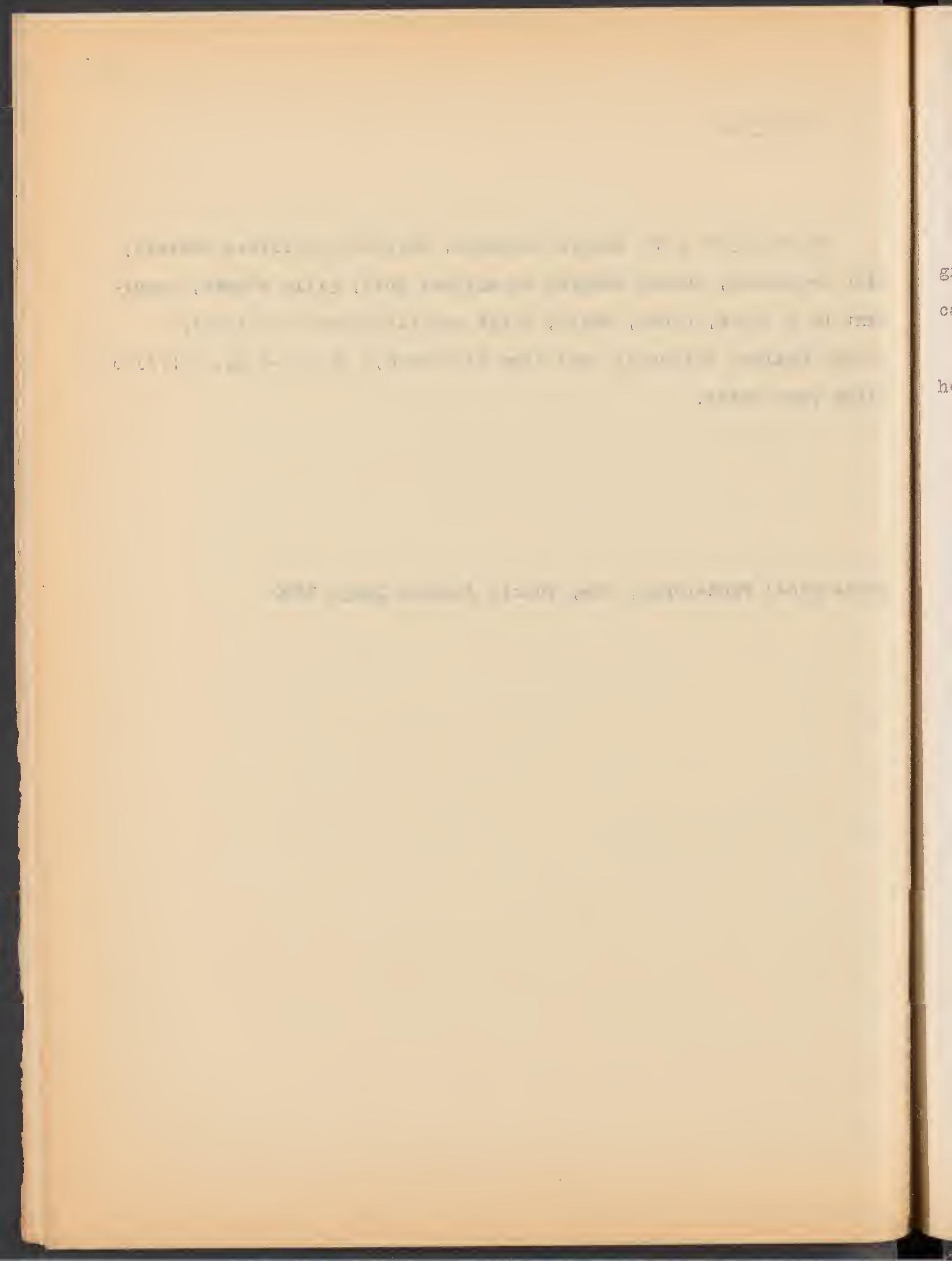


Collybia

Pileus $2.5 \mathrm{~cm}$. cartridge buff umbonate, margin finely striate, moist gills close, adnate, white, medium width; stipe $5.5 \mathrm{~cm} \times 5 \mathrm{~mm}$, concolor cartilaginous, hollow, fragile.

heredia, Chahuites, $1600 \mathrm{~m}$., Goerger

$9-11-36$ 



\section{Collybia (new) Bomama group}

Pileus cespitose, campanulate, umbonate, white or nearly so, flesh smooth, thin; gills very narrow, crowded; stipe long slender, sveral often coalescing to form a pseudo-branched stipe; disc lightly tinged with brown.

\section{Specimens expmined:}

Costa Rica, Cartago, Santiago, Canon Reventazon,Alt.1020m. Doage \& Thomas 4643 

Collybia near albidulus.pat.

On $\log$ over arainage aitch.

Specimens examined:

Costa Rica, San Jose, San Pearo de Montes de Oca, Alt. 1200 
Collybia

Pileus up to $3 \mathrm{~cm}$, expanded, pure white, margin irregular very thin not stiate; gills white, adnate, subdistant, narrow; stipe $2.5 \mathrm{~cm}, x 4$ mm, compressed, white cartilaginous

Decaying 108

Limon, Siquirres, Castilla, $20 \mathrm{~m}$., Dodge \& Goerger 
Collybia

Pileus 4cm., flat, almost membranous, coarsély wrinkled, white or very slightly pinkish; margin faintly striate

Gills close, narrow, ventricose, free or very slightly sinuate adnexed, white

Stipe 12cm. $x$ 4mm., hollow, cartilaginous, fibrillose, cottony white

On leaf mould

Costa Rica, San José prov, Paquita hill, $20 \mathrm{~m}$. 


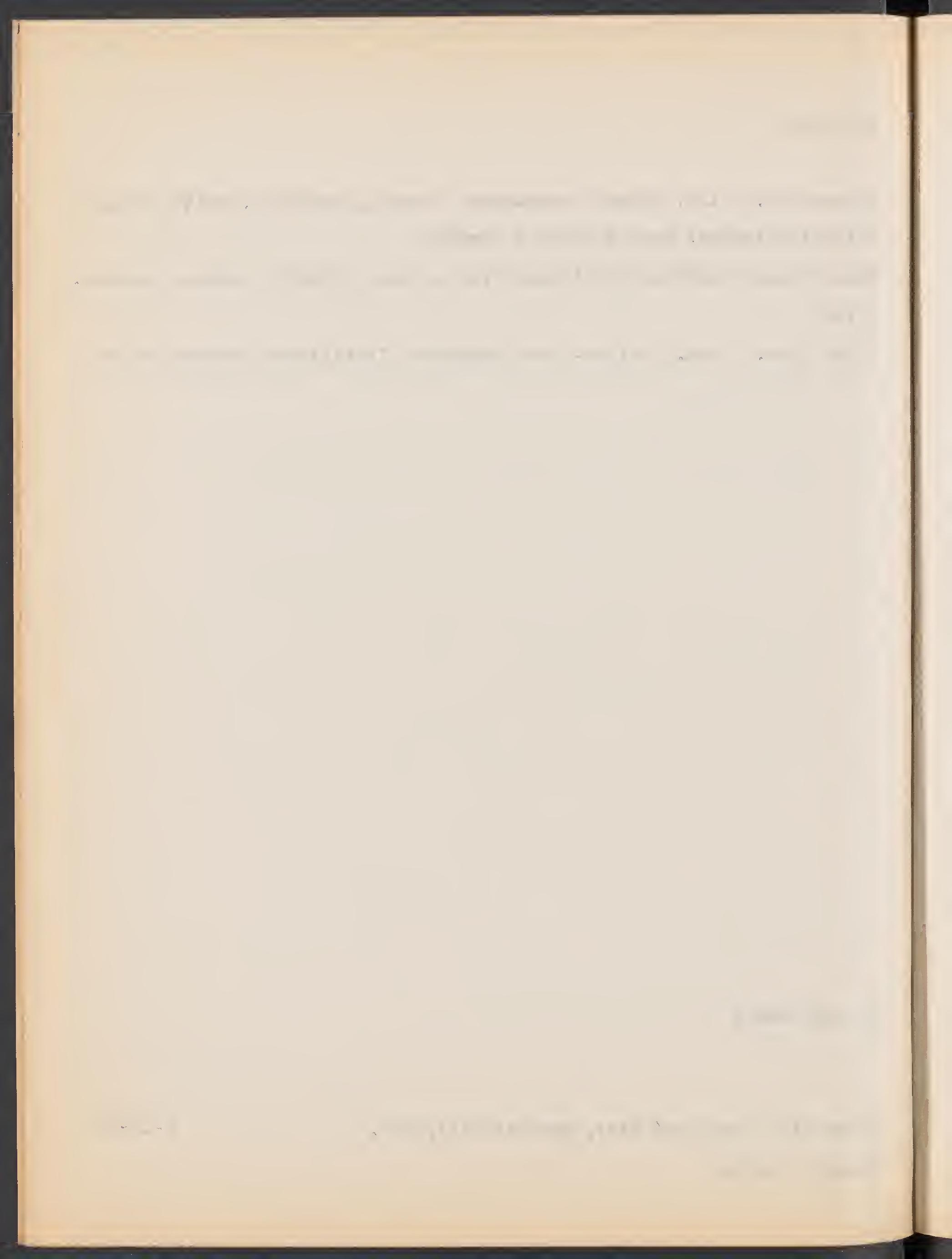




\section{Collybia albidulus group}

Pileus convex, center depressed, $6 \mathrm{~cm}$ in diameter, deeply sulcate striate from splitting over gills, cartridge buff, gills broad, m distant, white, adnate, stipe white, minutely velvety $6 \mathrm{~cm} \times 4 \mathrm{~mm}$

Limon, Siquirres, Castilla, $20 \mathrm{~m}$., July 25 Dodge \& Goerger 


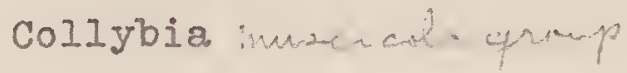

Pileus $1.8 \mathrm{~cm}$, white, center deeply umbilicate, convex, smooth; gills adnate, broad, close white stipe cartilaginous white $2 \mathrm{~cm} \times 2 \mathrm{~mm}$.

Decaying $\log$

Simon, Siquirres, Siam, $20 \mathrm{~m}$. Dodge \& Goerger $7-30-36$ 

Collybia near cyanocephajus

Pileus $5 \mathrm{~cm}$, surface fibrillose, moist, pellicle separable, margin deeply lacerate, dusky Ereen gray; gills narrow, crowded subdecurrent, white; stipe concolor, smooth, $4 \mathrm{~cm} x 4 \mathrm{~mm}$, hollow

Costa Rica: Puntarenas, Osa Fstero sandalo 2-20 m, Dodee 7670 


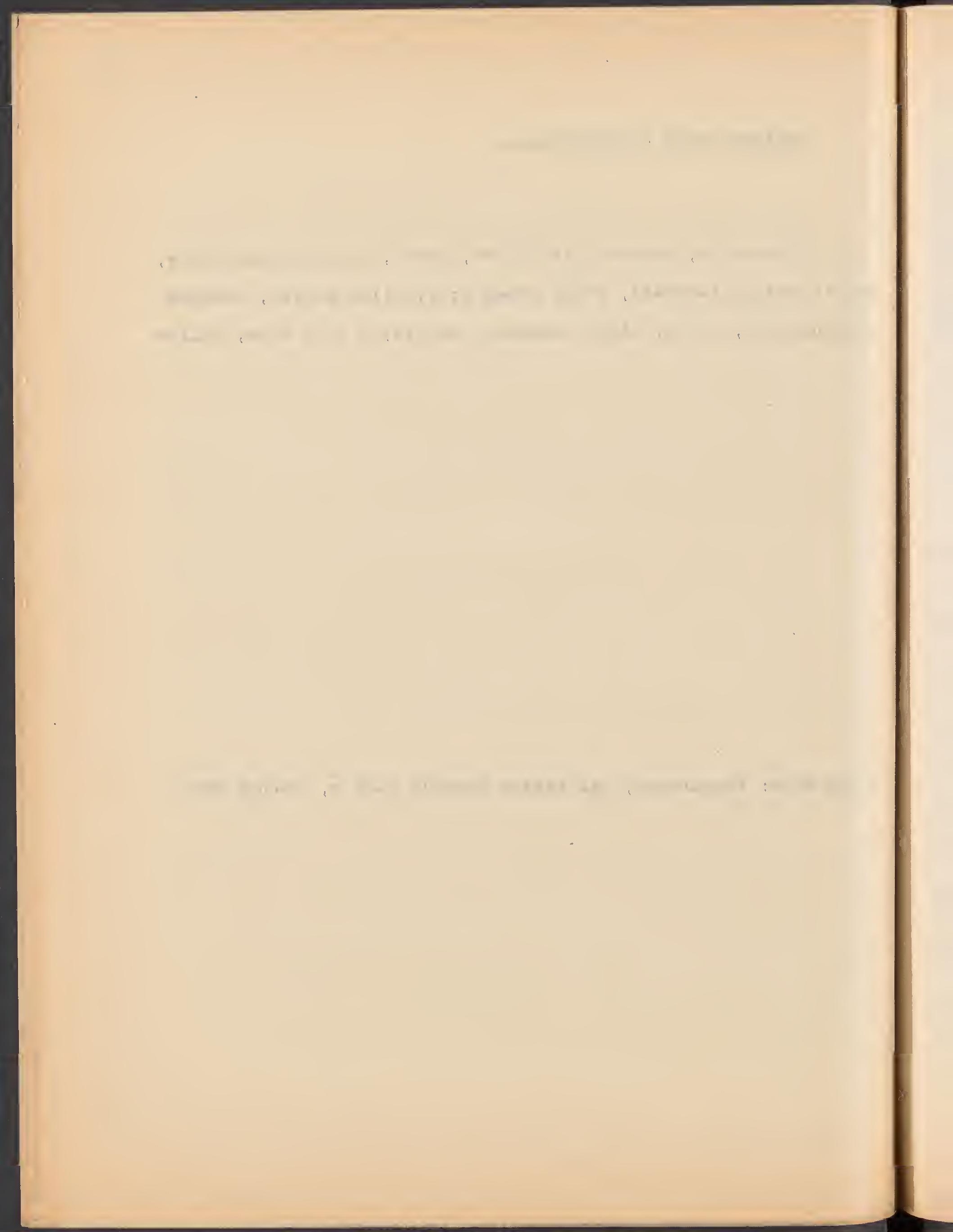


CoLlybia

Pileus expanaed, thin up to $5.5 \mathrm{~cm}$. lime-green with

disc tawny or lighter, somewhat tawny where bruised, subhygrophanous; flesh very thin; gills, narrow, crowded, concolor;

stipe silky, concolor, becoming tawny where bruised, up to $5-6 \mathrm{~cm}$. Long.

Dead log.

Specimens examined:

Costa Rica, San Jose, Rancho Redondo, Alt. 2500m. Dodge \&Thomas 4983 

Collybia.

Pileus dull lavender to almost dark lavender, hemispheric to expanded with fine anastomosing stria except on disc, $2 \mathrm{~cm}$. gills adnate, close, narrow, white or nearly so; stipe $7 \mathrm{~cm} \times 2.3 \mathrm{~mm}$, cartilaginous hollow, very minutely velvety, concolor, dull lavender

Heredia:

Chahuites, 1600 m., Goerger 


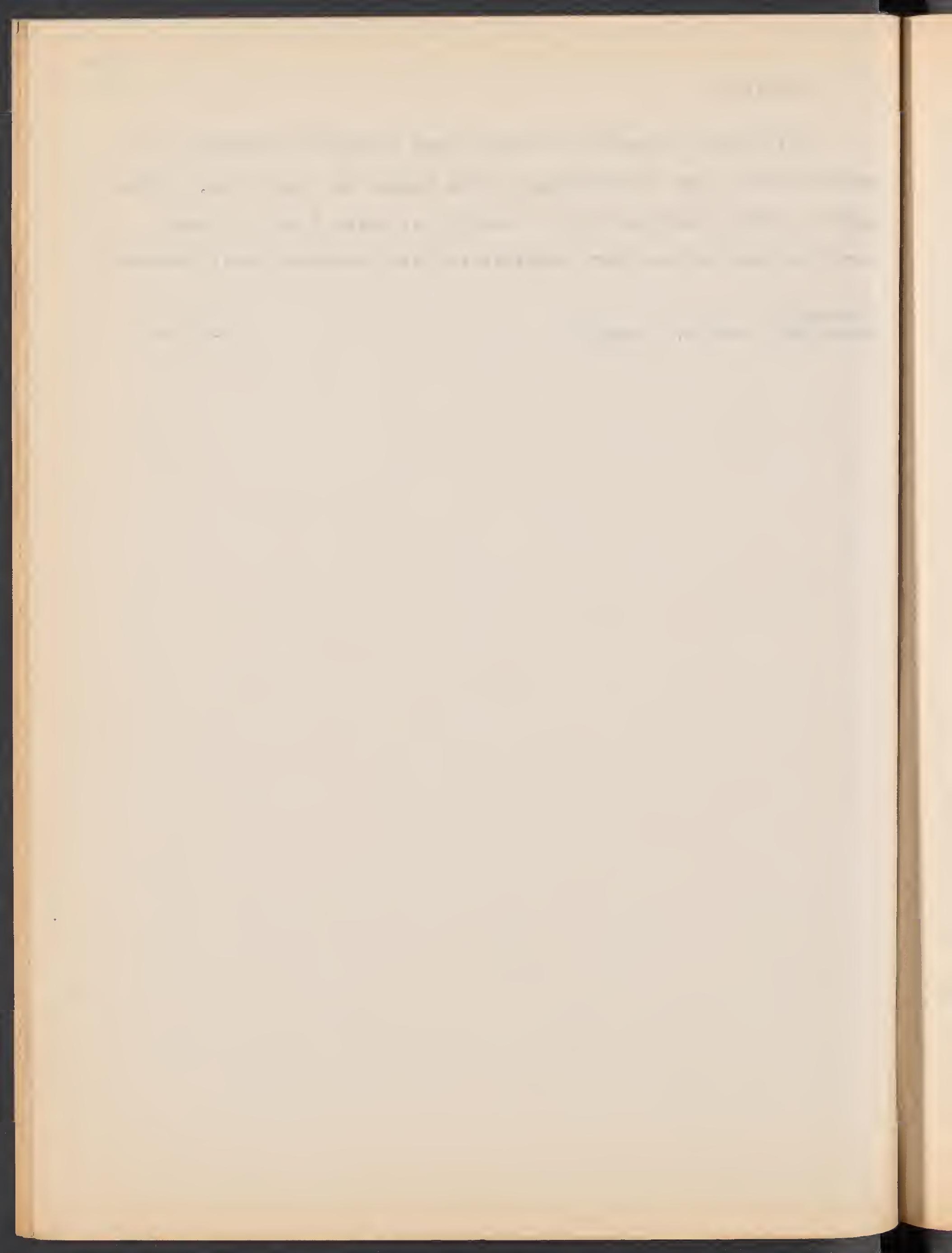


Collybia hear niquita

P1leus convex, expanded to irregular, up to $4.5 \mathrm{~cm}$., bistert to biack above, hygrophanous, margin thin, smooth; Gills adnate, white, blackening where bruised, close ventricose; stipe cartilaginous up to $3.5 \mathrm{~cm} \# 3.5 \mathrm{~mm}$., turfuraceous, pruinose-buff but quickly blackening, hollow.

Potrero S. of House

Specimens examined:

Costa Rica, San Jose, Rancho Redondo, Alt. 2160-2180. Dodge \& Rosettill above Rio Pacume Nodge Catt Thomas et al. Puntarenas: Osa, R. Jerrones $2 \mathrm{~m}$ sodge 
sI

$c 2$

or

Co 
Collybia

Pileus $3 \mathrm{~cm}$, cinnamon rufous, umbo laree, margin upturned, sliuhtly lacerate, eills adnexed tofree, white, crowded, broad; stipe cartilaginous, $5 \mathrm{~cm} \times 2 \mathrm{~mm}$, white above, fibrillose, cinnamon rufous or liehter below

Costa Rica: Puntarenas, Osa, Fstero Sandalo 5-20 m Dodee 7732 
$\mathrm{Pi}$

Mi

Gi

st

$\mathrm{Co}$
Do 
Collybia

Pileus 2am., convex, broadly unbonate, pale ochraceous salmon; marlin. Margin somewhat lighter, innate fibrillose, striate

Gills white, narrow, crowded, adnate

Stipe $7-8 \mathrm{~cm} .72-3 \mathrm{~mm}$, pruinose, white

Costa Rica, Limón prov., Castilla farm, $20 \mathrm{~m}$.

Dodge \& Goerger 
wh

cas

Cos 
Collybia

Pileus up to $3.3 \mathrm{~cm}$, convex, sliehtly umbonate, honey yellow wholly, margin striate; sills white, close adnexed, narrow; stipe cartilacinous, compressed, $7 \mathrm{~cm} \times 3 \times 1.5 \mathrm{~mm}$, white.

Costa Rica: Yuntarenas, Osa near R. Terrones, 2U-30 m, Jodge 7671 

Coilybia near cinchonensis

Pileus $2.5 \mathrm{~cm}$ with deeply depressed, slight umbo, deeply striate sulcate, tilleul buff; cills adnate-adnexed, distant, medium, tilleul buff; stipe pruinose, fibrillose, $6 \mathrm{~cm} \times 2.5 \mathrm{~mm}$

Costa Rica: 'Juntarenas, Osa, near R. Terrones $10 \mathrm{~m}$ Dodge 7696 near Estero Sandalo 5-20 m, Dodge 7685, 7733; nearPuerto Jimenez 5-20 m, 7642; Cerro Guaca Dodge 7639 
Collybia

Pileus vinaceous fawn, $3 \mathrm{~cm}$, expanded, faintly umbonate, striate, hygrophanous; gills adnate broad, subdistant, paler than pileus; stipe $5.5 \mathrm{~cm} \times 3.5 \mathrm{~mm}$, cartilaginous hollow, silky fibrillose

Heredia, Chahuites, 1600 m., Goerger $9-11-36$ 

Pileus broad campanulate, subconic, $3 \mathrm{cr}$ in diameter, $1.5 \mathrm{~cm}$ tall, striate sulcate, smooth, dry, disc mummy brown or darker, shading to buckthorn brown, gills subdistant, adnate, broad, mustard yellow to Naples yellow, edges smooth; stipe $4 \mathrm{~cm} \times 3-5 \mathrm{~mm}$ tapering upward, cartilaginous tough, velvety, raw sienna above to raw umber below

Decaying stick in bananal

San José, R. Paquita, 2 m., Dodge \& Goerger Aug. 14 

$\operatorname{col} I y b i a$

Pileus broad, campanulate, $1.5 \mathrm{~cm}$, hygrophanous, pinkish buff with mars brown margin, smooth, fibrillose, flesh thick on disc, thinning rapidly, white; gillas white at first becoming pinkish cinnamon, adnate, close very narrow; stipe cartilaginous, hollow, silky fibrillose, white hygrophanous.

SanJosé, Guayabillos, $2300 \mathrm{~m}$. , Dodge \& Goerger 


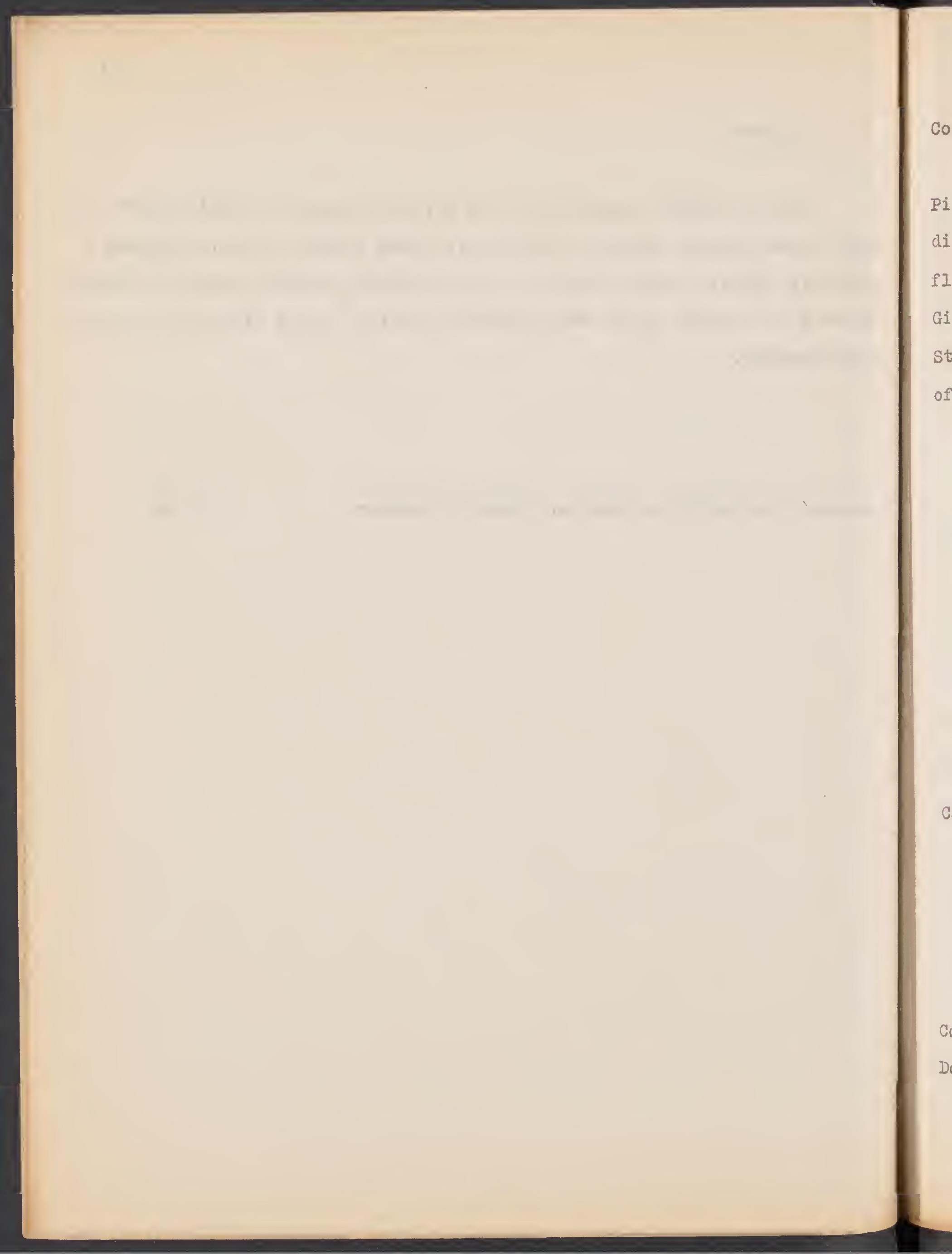


$\operatorname{Col1ybia}$

Pileus 3-4cm., campanulate, expanded, moist but not truly viscid; disc cinnamon brown to ochraceous tawny; margin not striate; smotth flesh very thin

Gills close, narrow, adnate, light ochraceous buff

Stipe 1I-12cm. $x$ 2mm., Hessian brown, smooth, cartilaginous, Iower $3 \mathrm{~cm}$. of base clothed with whitish tomentum

Caespitose in decaying leaf mould, in forest along quebrada

Costa Rica, San Jose prov., Guayabillos, 2300m. 
Collybia

Pileus convex, $1.5 \mathrm{~cm}$, disc chestnut shadineto cinnamon rufous margin, inrolled; eilzs adnate, close, hroad, citrine pyellow; stipe cinnamon rufous $3 \mathrm{~cm} \times 2-1 \mathrm{~mm}$ tempering downward

$$
\text { Rotten Iog }
$$

Costa Rica:limon, Marta $20 \mathrm{~m}$. Dodge 7253 


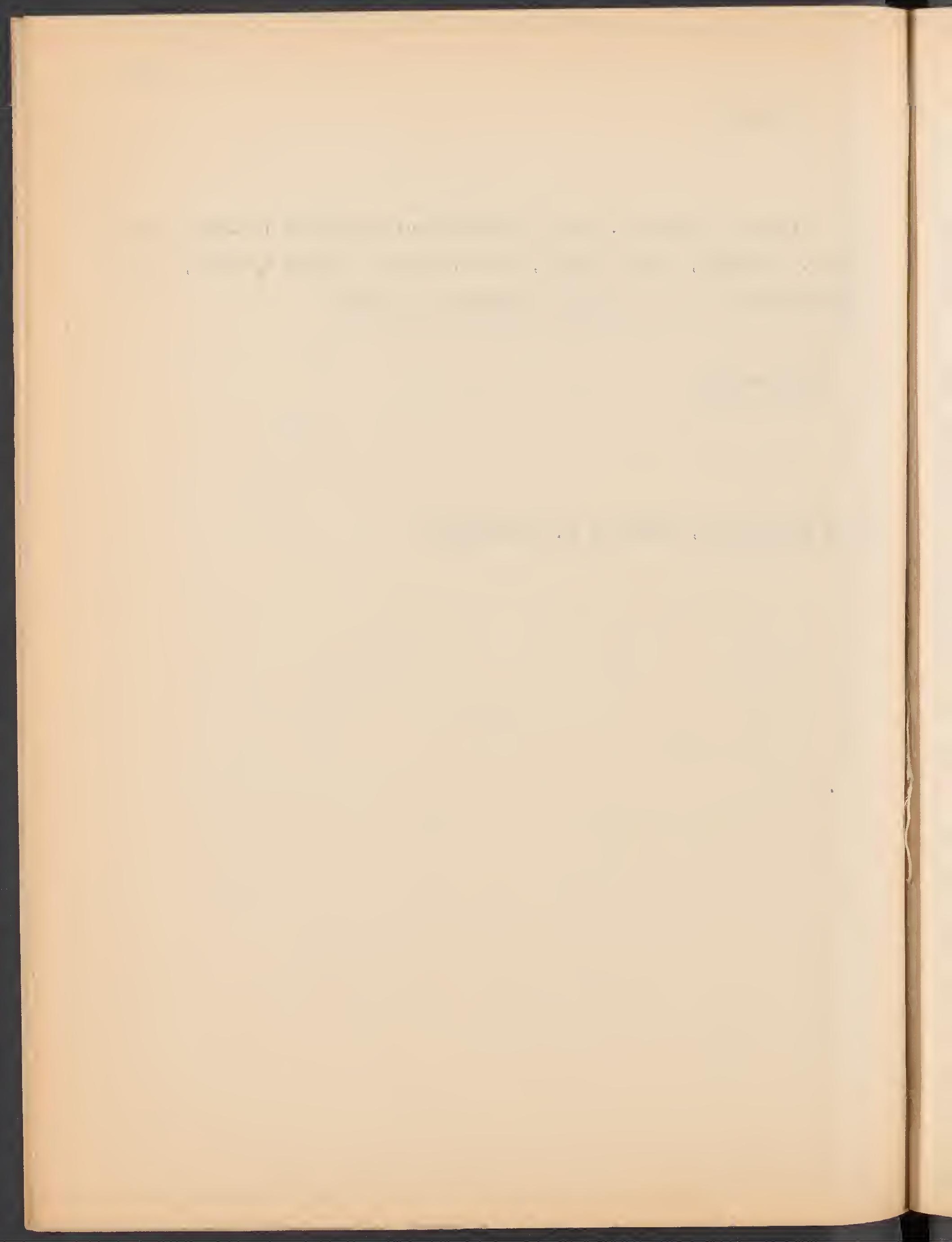


Collybla

Pileus whitish, tawny on disc, slightly hygrophanous, dimeter about 1 in. on largest; gills narrow, close, attachea to stipe,flesh thin; stipe thin, brittle, slightly porous.

In clusters on wood in jungle

Specimens examinea:

Costa Rica, Cartago, Pejivalle, Alt. $580-800 \mathrm{~m}$. Doage \& Thomas 4427 
Hiatula

Collybia

pileus expanded, $5 \mathrm{~cm}$, very thin, russet with darker disc and lighter margin whjch is broadly stiate sulcate; gills adnate, sinuate, ventricose, thick, subwaxy, tilleul buff, spores white, stipe hollow, cartilaginous silky russet, $2.5 \mathrm{~cm}$ s $3.5 \mathrm{rm}$ ending in bulb up to $7 \mathrm{~mm}$

On log by roadside

Specimens exumined:

Costa Rica: San Jose, Rancho Redondo 2300-2350 m Dodge \& Thomas 5387 


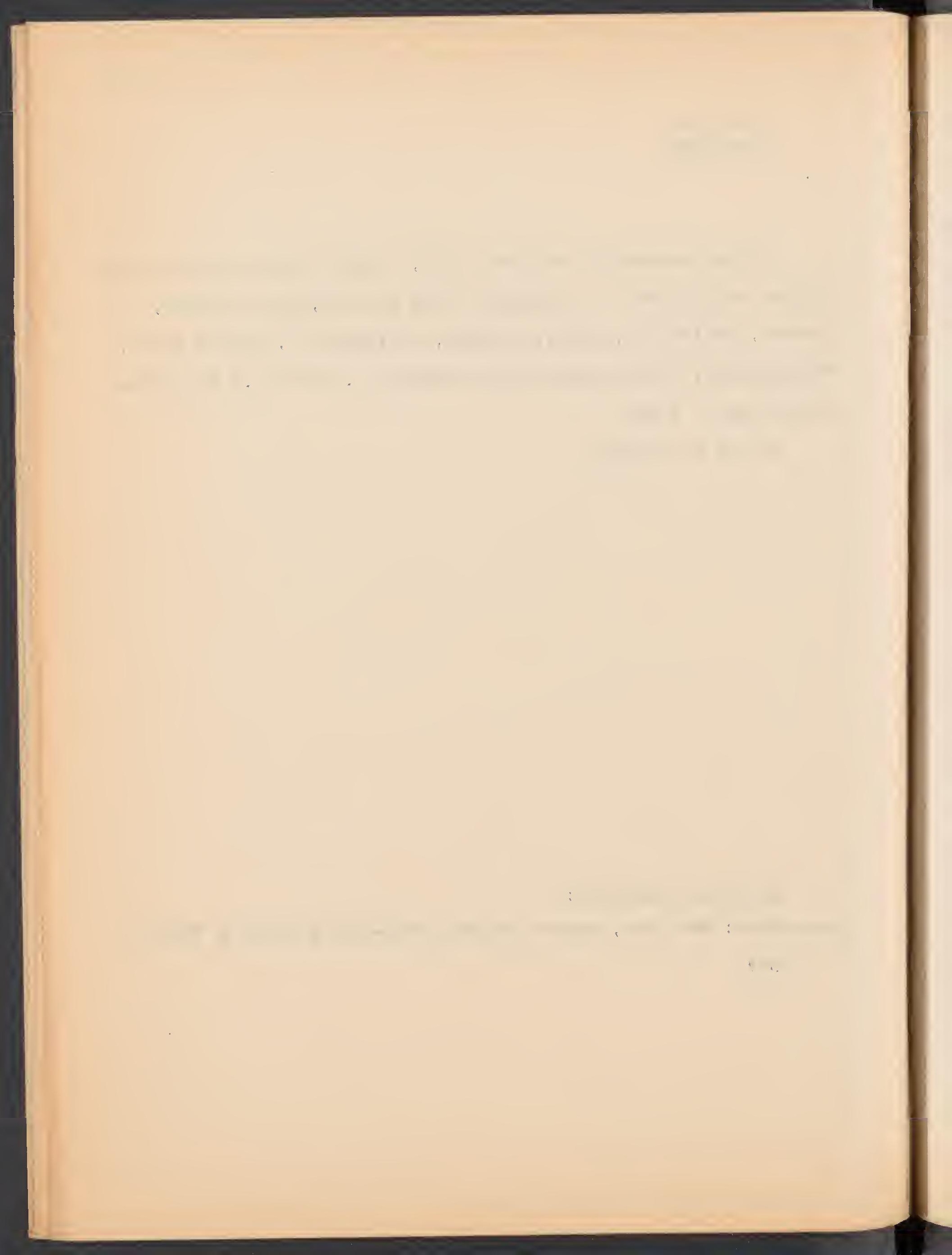


Collybia

Pileus convex, $2.5 \mathrm{~cm}$ [watersoaked and old] margin smooth sulcate striate to disc, russet shading to tawny or lighter; gills groad, adnate pale ochraceous salmon, stipe ochraceous salmon, $4 \mathrm{~cm} \times 2 \mathrm{rm}$, equal, cartilaginous

Cespitsose on very rotten log, forest along quebrada

San José, Guayabillos, $2300 \mathrm{~m}$. , Dodgę Goerger $7-10-36$ 


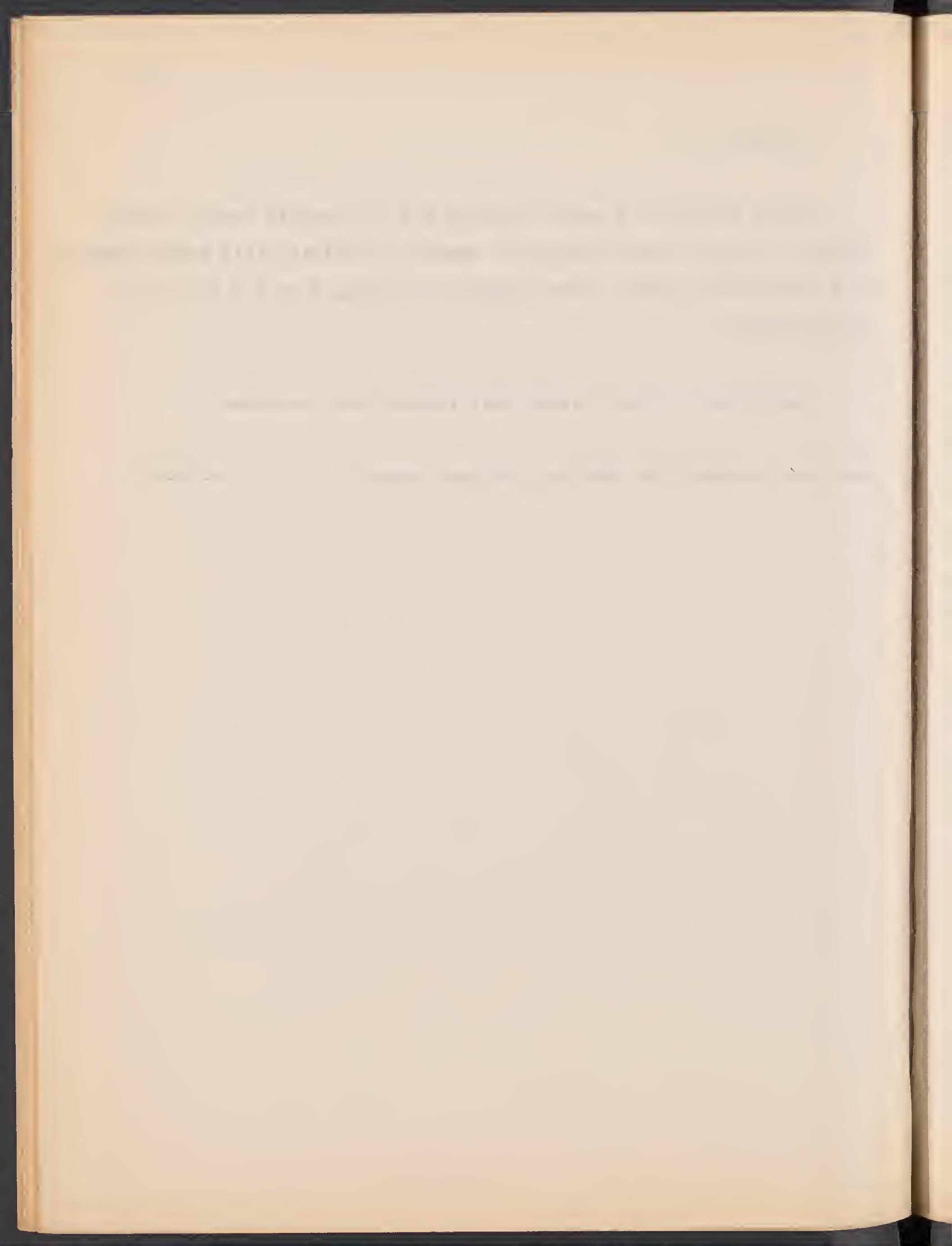


Collybia

Pileus expanded, cespitose up to $6 \mathrm{~cm}$, irregularly gibbous, mars brown or russet on disc, hygrophanous, striate sulcate, color deeper over gills, margin irregular, sinuate white; flesh thin; gills ventricose, adnate with decurrent tooth, subdistant white; stipe white above, pale cinnamon pink below, pruinose toward pase, up to $5 \mathrm{~cm} \times 4 \mathrm{~mm}$.

Cespitose on very rotten log

SanJosé, Guayabillos,

m., Dodge \& Goerger

$7-10-36$ 



\section{$131-13$,}

Collybia

Pileus convez, hygrophanous, striate over gills, $3 \mathrm{~cm}$; disc light vinaceous cinnamon: rest Mikado brown; margin irregular Gills distant, white, adnate to collar about stipe Stipe pruinose above, cartilaginous, hollow, 2mm. diameter $\times 4 \mathrm{~cm}$., light vinaceous cinnamon

Costa Rica, Puntarenas prov., Osa, R. Sándalo, 1-5m. $8-21-36$ Dodge \& Goerger 

Collybia

Tricholma

Pileus hemispheric to expanaed, margin unrolied as in Lactarius, suriace smooth, moist, ochraceous-tawny with cinnamon-brown flesh pale-buft, thick on disc, thin at margins, floccose; gills sinuate, adnexeü, ventricose, white,close;

stipe separable frow fllesh, hollow, cartilaginous, citrine-yellvw within, white above, ochraceous-tawny below.

On stump.

\section{Specimens examinea:}

Costa Rica, San Jose, Rancho Redondo, Alt.2500 m. Dodge \& Thomas 

Collybia

Pileus convex to nearly hemispheric, $2.5 \mathrm{~cm}$ striate sulcate on margin along gills somewhat hygrophanous, center Sayal brown rest Iight pinkish cinnamon, distant, adnate broad; stipe $6 \mathrm{~cm} \times 2.5 \mathrm{~mm}$, tapering doward, vinaceous buff

Limon. Siquirres, Castilla, $20 \mathrm{~m} .$, Dodge \& Goerger 

Collybia

Pileus 3 cm expanded convex, margin short striate, thin, slightly lacerate, Naples yellow, shading to white; gills adnate, close, narrow white; stipe $3 \mathrm{~cm} \times 2.5 \mathrm{~mm}$, white cartilaginous

\author{
Decaying $\log$
}

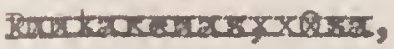

Limon, Siquirres, Castilla, $20 \mathrm{~m}$., July 29 Dodge \& Goerger 


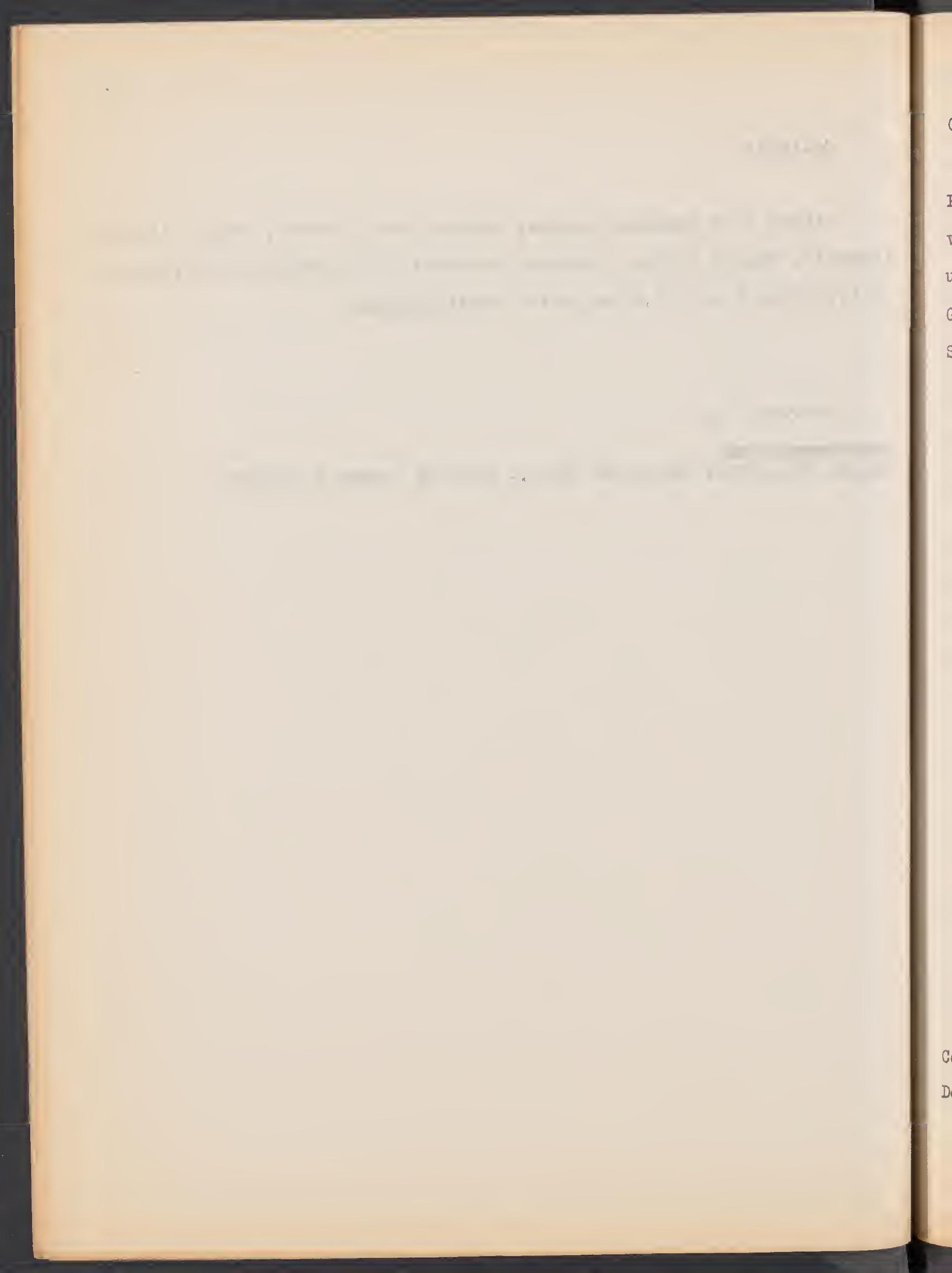


Collybia

Pileus 2.5cm., hygrophanous; margin striate sulerte, expanded; disc Pinaceous cinnamon shading to pale cinnamon pink; margin white, somewhat undulate

Gills ventricose, adnexed, subdistant, white Stipe cartilaginous, white, $3.5 \mathrm{~cm}, x 2.5 \mathrm{~mm}$, hollow

Costa Rica, Puntarenas prov., 0sa, R. Sándalo, I-5m. $8-21-36$ Dodge \& Goerger 
Coifyida vcuiatus group van 4427

Pileus varyine from $1.5-6 \mathrm{~cm}$ diam, moist, hyerophanous, disc tawny to Mars brown, margin light buff, striate or sometimes whole pileus pinkish cinnamon; gills adnate, subdistant, narrow, white, stipe $3-15$ an $x$ 2-4 mm, smooth, tawny to pinkish cinnamon Greerrious to cespjotose 

Collybia.

Pileus hygrophanous, up to $3 \mathrm{~cm}$., margin faintly striate; disc yellow ochre, rest wax bellow

Gills crowded, narrow, adr te to very slightly decurrent, amber yellow Stipe cartilaginous, tough, silky, wax yellow, $4 \mathrm{~cm}, x 1.5 \mathrm{~mm}$, on buttress roots of standing tree in forest (see photo) 


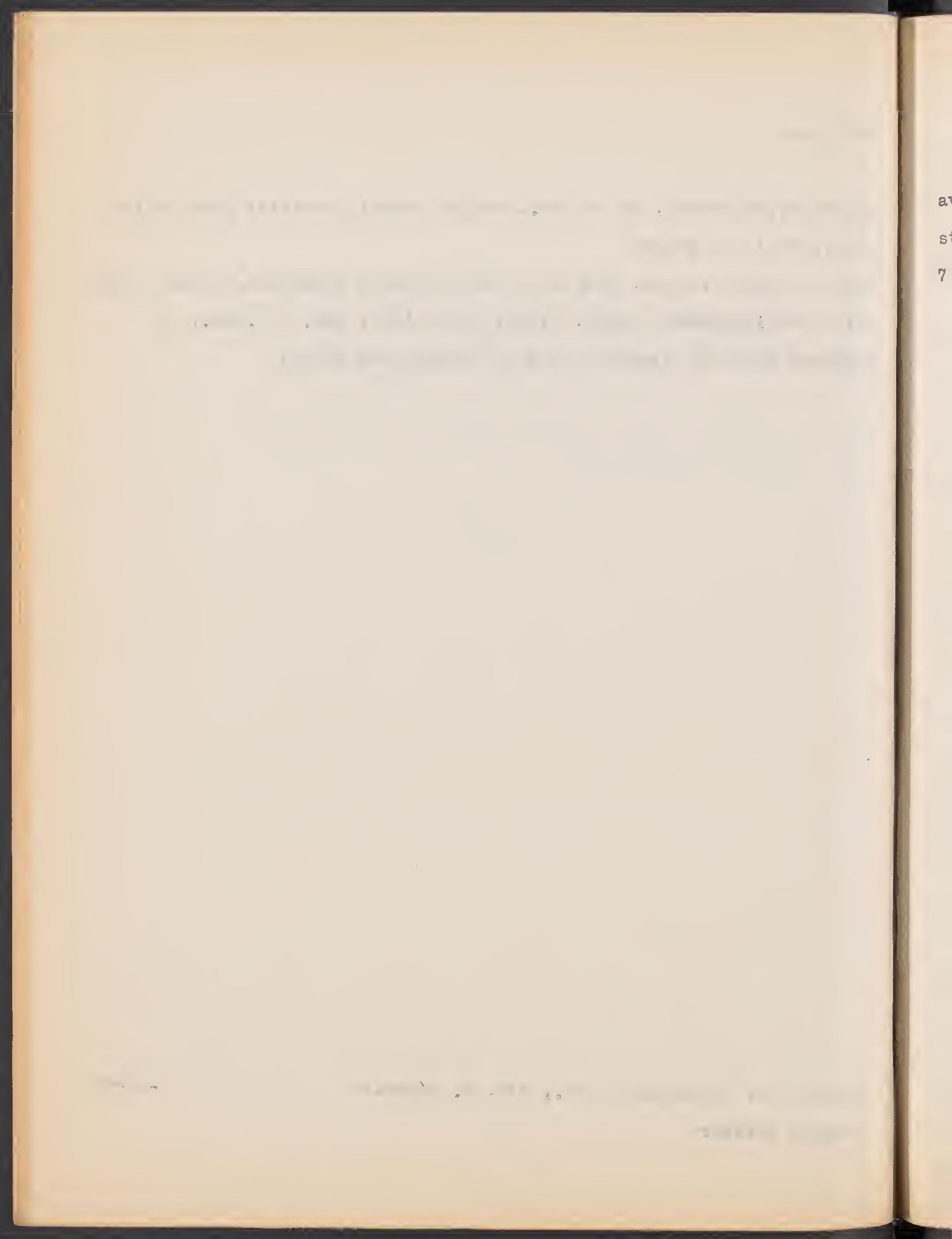




\section{$17 \times-17$ \\ 153}

Collybia

Pileus flat, irregular $5.5 \mathrm{~cm}$, margin striate sulcate, lighter center, avellaneous to vinaceous buff; gills subdistant, adnate, broad, white stipe compressed, white cartilaginous $5 \mathrm{~mm}$ above taperingto $2 \mathrm{~mm}$ below $7 \mathrm{~cm}$ long

Limon, Siquirres, Castilla, $20 \mathrm{~m}$. Dodge \& Goerger $7-25$ 


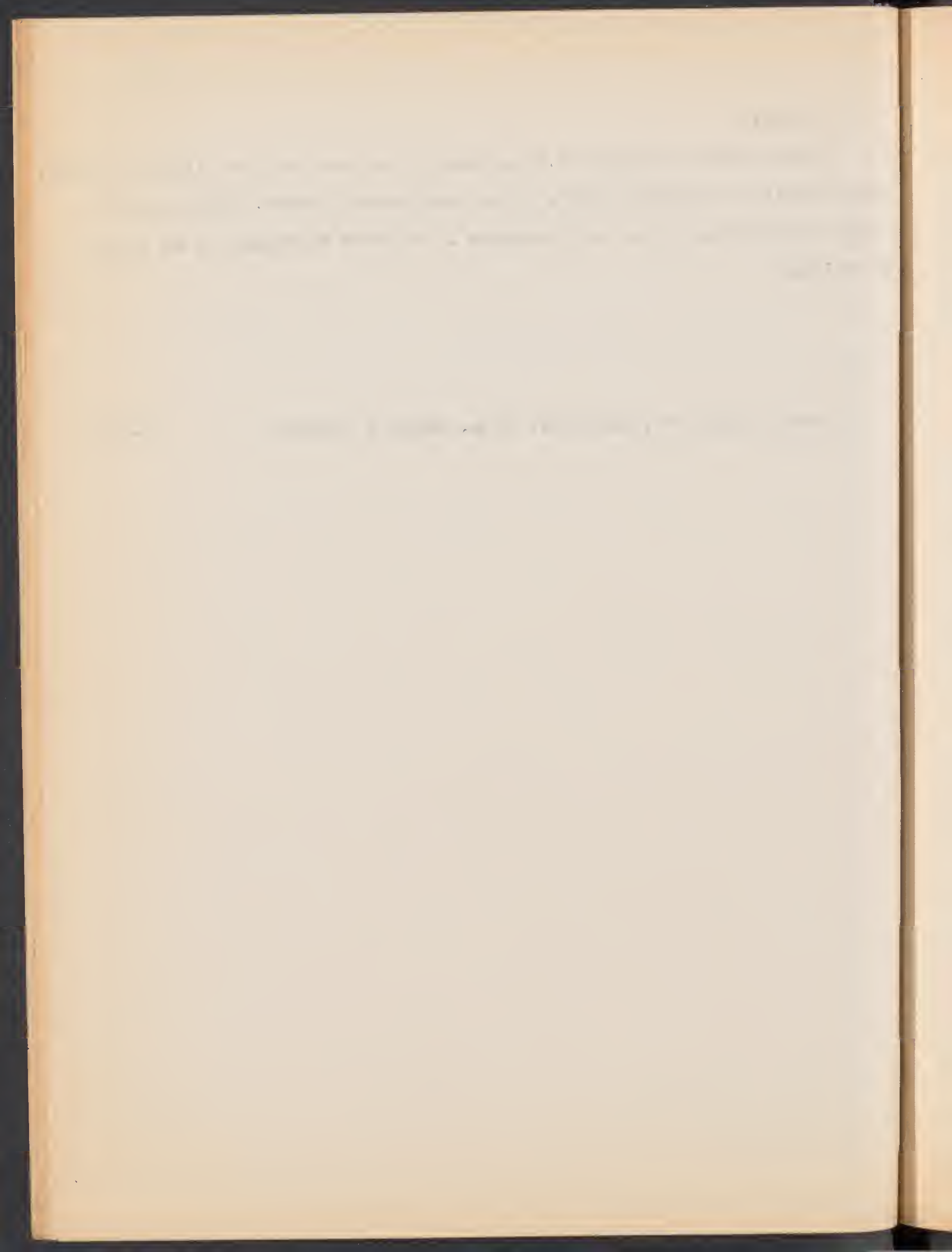




\section{Collybia near C. oculatus \\ c. monicila}

Pileus $3 \mathrm{~cm}$...smooth, pale ochraceous-salmon with russet umbo, margin sulcate striate; flesh very thin, white; sills white, anate with decurrent line, broad; stipe concolor or darker with cottony white mycelium, slenaer equal $4 \mathrm{~cm}$. Iong, $3 \mathrm{~mm}$. diameter.

no agrees with above but pleus depressed sholthy umbilucate qills

\section{Specimeng examinea:}

Costa Rica, Cartago, N.W.slope La Carpintera, Alt.1550 m.

Puntaremas: Osa, Ris Sandrval $5.20 \mathrm{~m}$ Dodge

Doage \& Thomas, 4810 


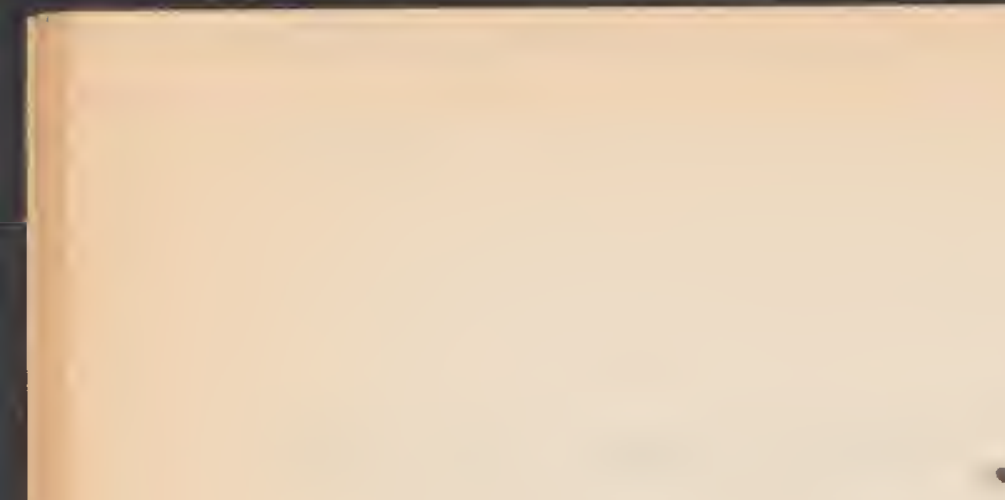


Collybia

Pileus $5 \mathrm{~cm}$, flat then edges upturned, moist not $v i$ said, decry sulcate striate over gills, disc between mummy brown and dresden brown shading kuktex to light buff, semipellucid, margins; gills rather thick, edges eroded, white, adnate, broad; stipe cartilaginous hollow, $7 \mathrm{~cm} x$ 4-2 $\mathrm{mm}$ tapering downward, Sayal brown except $1 \mathrm{~cm}$ at apex which is white

SanJosé, Santana, cañon R. Uruca, Dodge \& Goerger

$$
9-8-36
$$


Collybia

Pileus $2.2 \mathrm{~cm}$., center depressed, striate sulcate to disc, warm buff to light buff margin

Gills suibdistant, adnate, broad, white

Stipe 1.2cm. $x 1-1.5 \mathrm{~mm}$. , tapering downward, cartilaginous, concolor ous above, deeper below

on decaying liana

Costa Rica, Puntarenas prov., Osa, R. Sándalo, 1-5m.

Dodge \& Goerger 
C07. ybia

Pileus very thin, $4 \mathrm{~cm}$, vlane, sljihtly umbilicate, marein sulcate, wood brown shadinito fawn disc; tiljs narrow, crowded, adnate tilleul buff; stile tough, cetilaunous, $6 \mathrm{~cm} x$ \& 2 morn, white, cottony over fawn or darker.

Costa Rica: Iimon, Gastilla $20 \mathrm{~m}$, Dodee 7213 


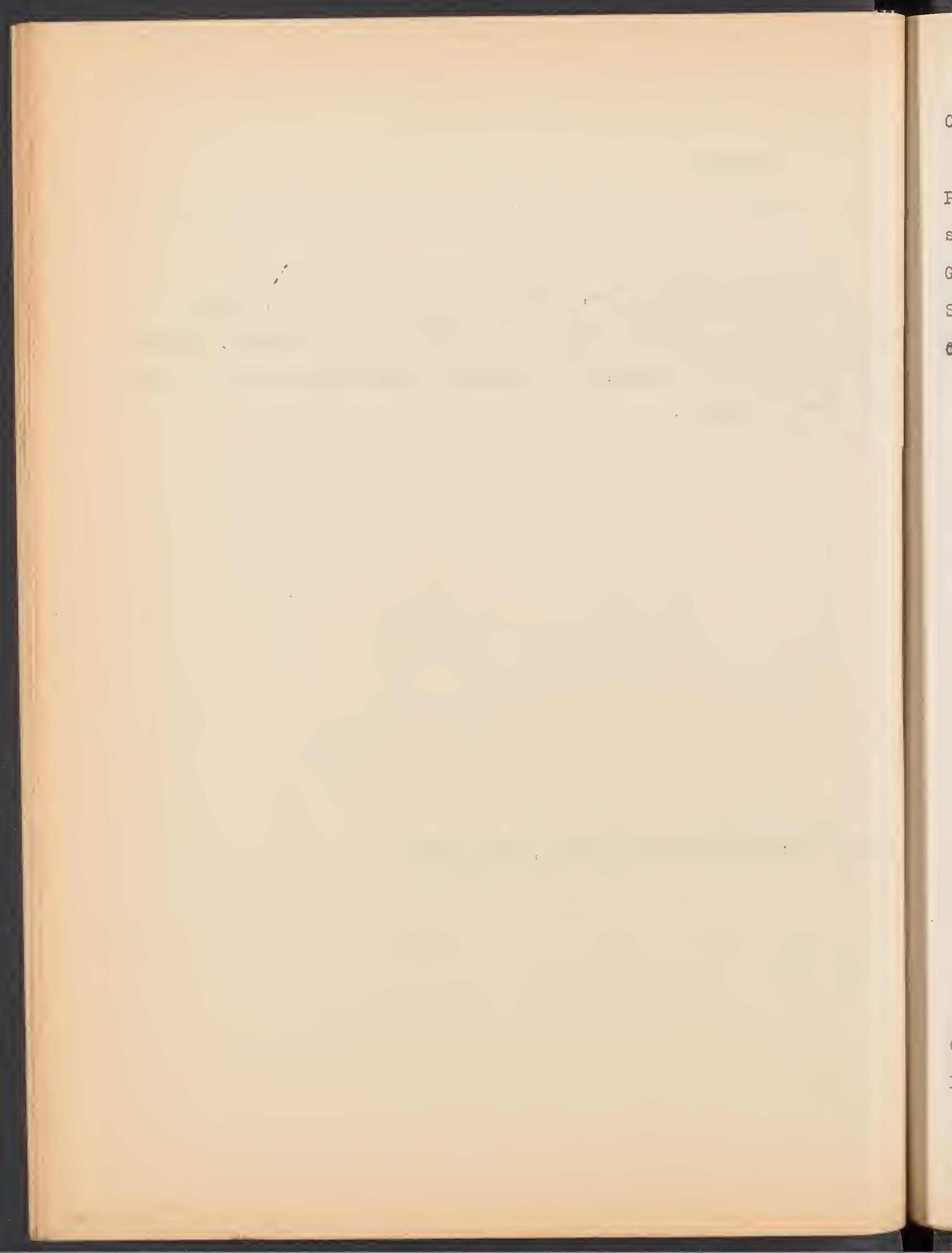


Collybia

Pileus $1.8 \mathrm{~cm}$, center depressed, margins elevated, sulcate striate, smooth; diac warm sepia, rest Verona brown

Gills adnexed to almost free, ventricose, white, close

Stipe cantilaginous, 2cm. $x .7-1.5 \mathrm{~mm}$., tapering downward, white above, Concolorous below

Costa Rica, Puntarenas prov., Osa, R. Sándalo, I-5m. 


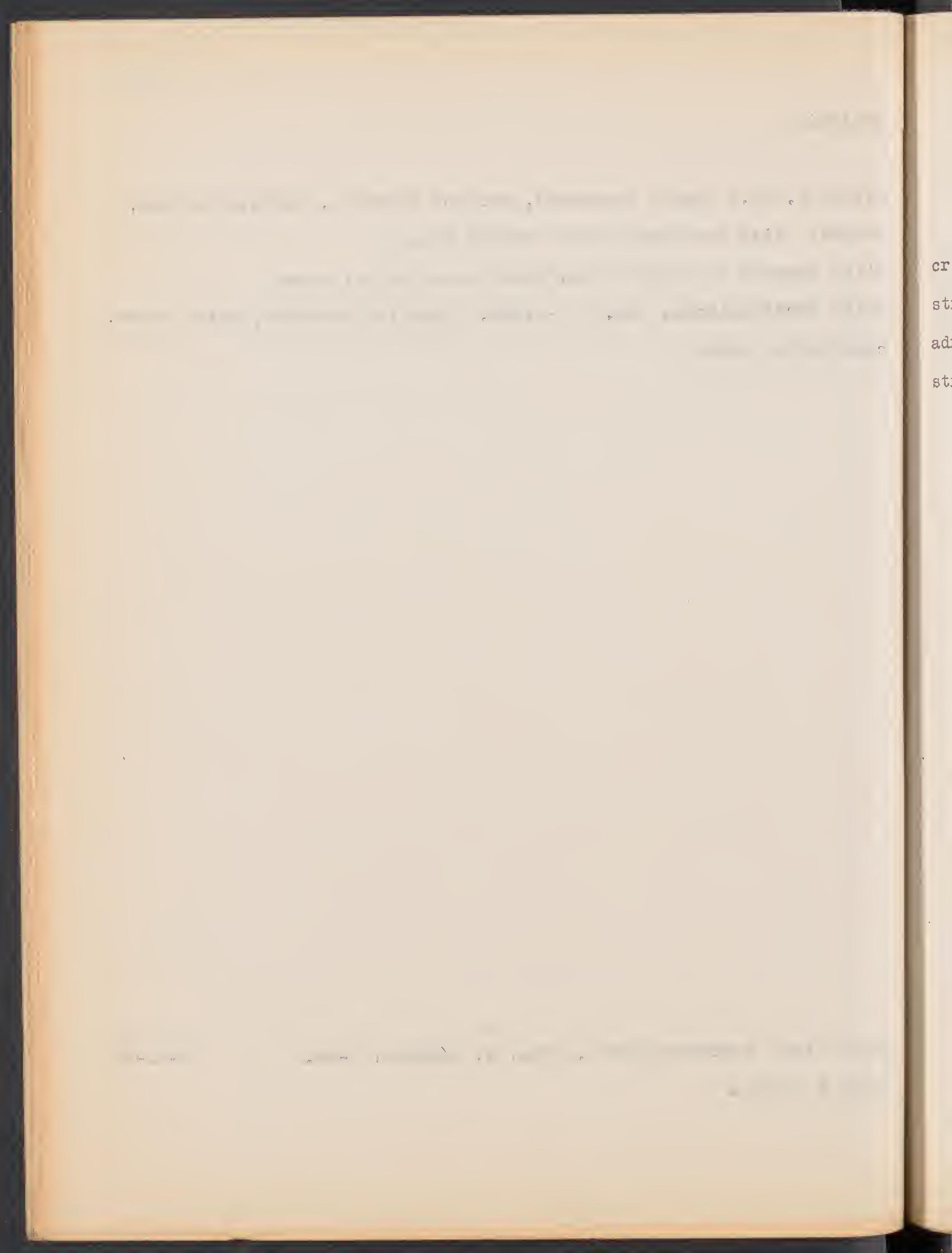


Collybia

Pileus very irregularly expanded, margins upturned and inrolled crisped, center depressed and umbilicate mikado brown to pinkish buff, striate, sulcate over gills, $7 \mathrm{~cm}$ in diameter; gills broad, distant, a adnate, pinkish buff; stipe hollow cartilaginous innate fibrillose striate, mikado brown or lighter $5 \mathrm{~cm} \times 8 \mathrm{~mm}$.

Puntarenas, 0sa, R. Sándalo $2 \mathrm{~m}$., Dodge \& Goerger $9-1-36$ 

Collybia

Pileus flat to slightly unbilicate with irregularly upturned margins, striate over gills to disc, $2 \mathrm{~cm}$, disc mikado brown, rest light Finaceous cinnamon, striate with Mikado brown; gills adnate to thickened apex of stipe but appearing free, white narrow close; stipe $4 \mathrm{~cm} \times 1.5 \mathrm{~mm}$, hollow cartilaginous tough, mikado brown, smooth Under Chusquea

SanJosé, Santana, cañon of R. Uruca, Dodge \& Goerger $9-8-36$ 
Collybja

Eileus $5 \mathrm{~cm}$, depressed, disc hazel shadine to antimony marein which is rugosestriate, hyerophanous; eills thick, broad, interveined. sinuate; stipe cartilakinous, hazel tapering downward, heavily tomentose $4 \mathrm{~cm} \times 4-2 \mathrm{~mm}$

In virein forest

Costa Fica:Limon, Wajceck $40 \mathrm{~m}$, Dodee $728 \mathrm{I}$ 


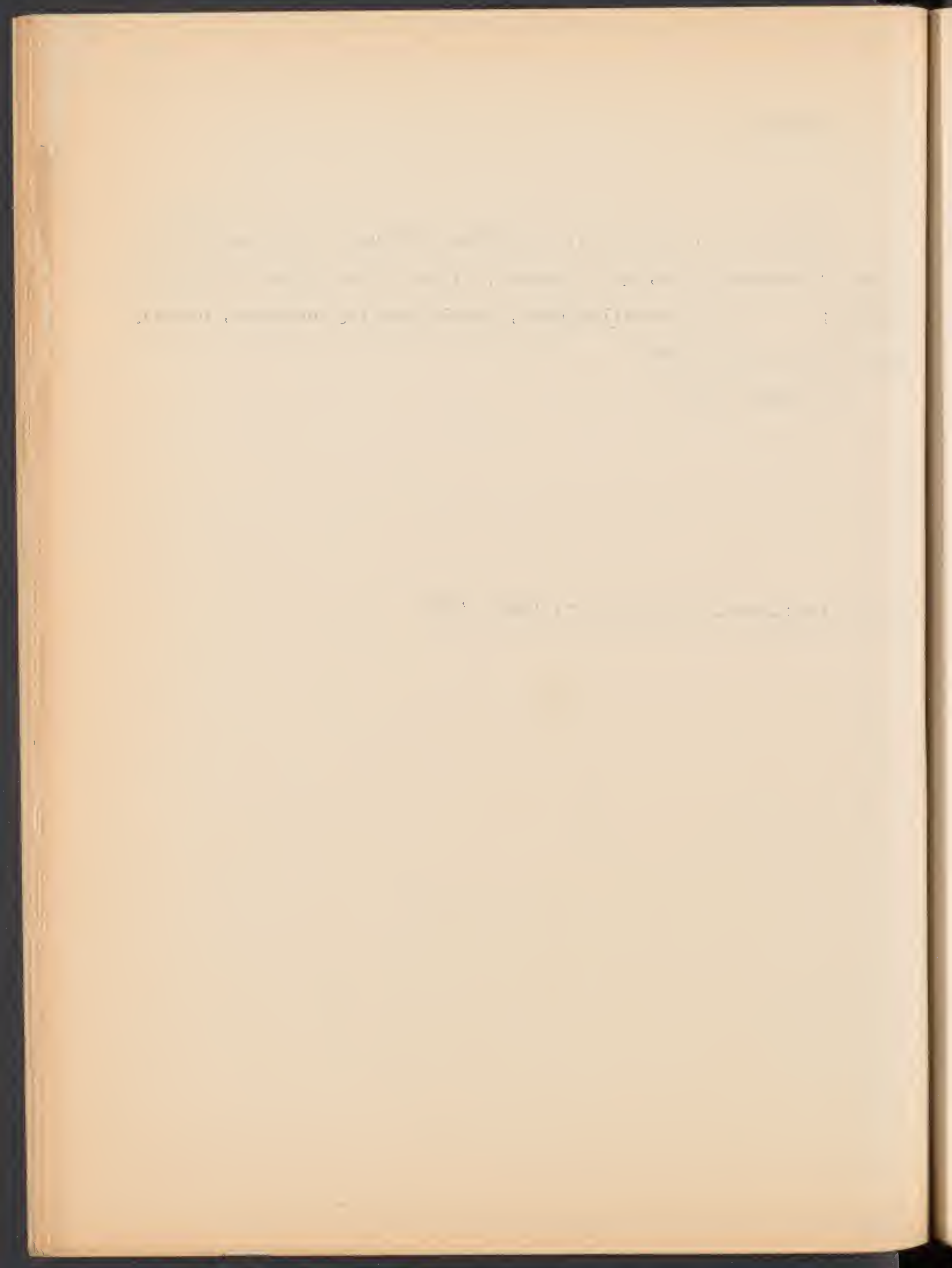

G

$D$ 
Collybia.

Pileus $2.3 \mathrm{~cm} .$, flat, center depressed; margin striate ober gills, cinnamon fufous to apricot buff

Gills distant, narrow, adnate, apricot buff

Stipe $2 \mathrm{~cm} . x$ 2mm., cartilaginous, hollow, smooth, apricot buff

Costa Rica, San José prov., cañon oî R. Uruca near Sta Ana, 


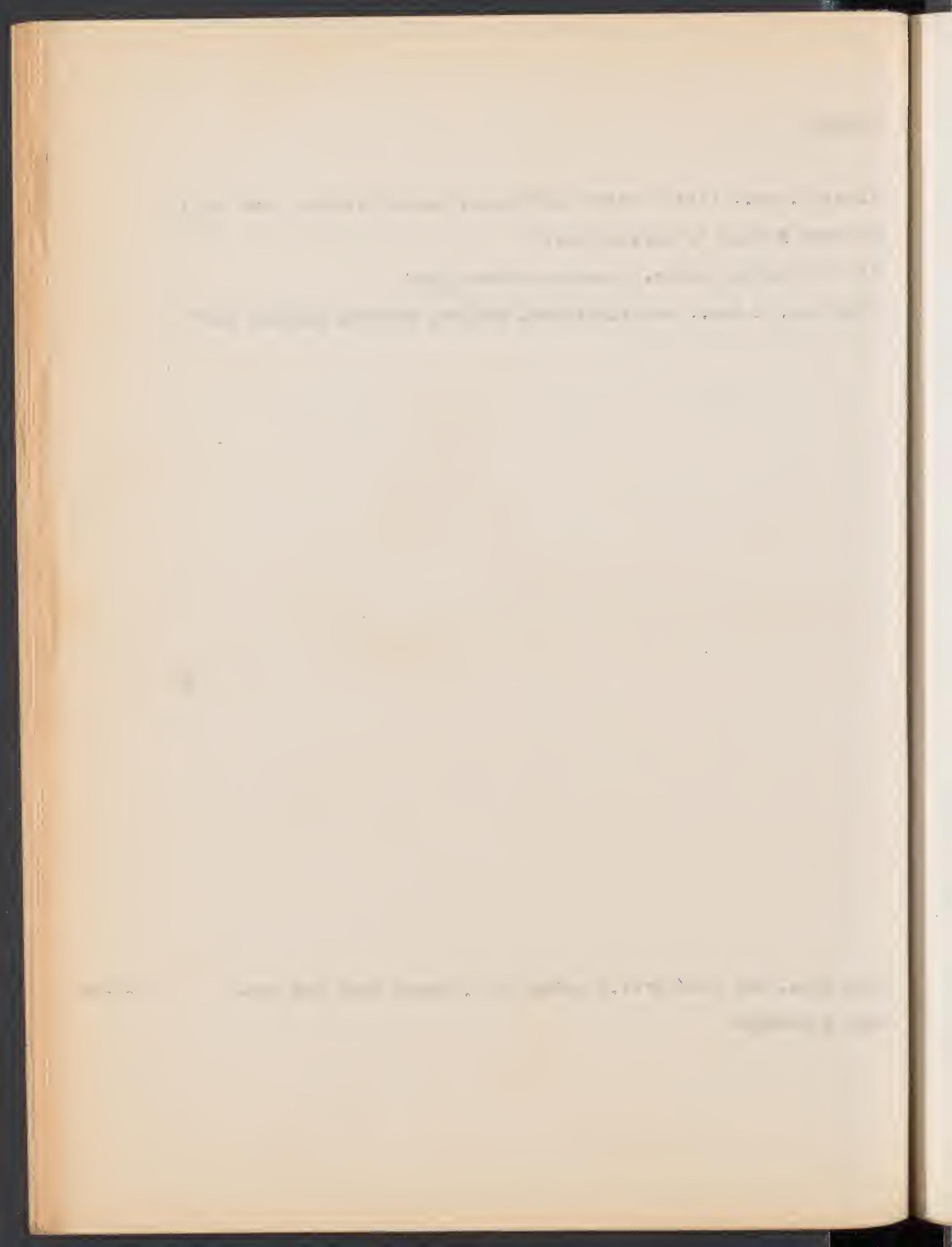


Collybia, large watery

?Hygrophorus. (see field notes on spec. same locality Nov. 6

On dead log in potrero south of house

Costa Rica: San José Province, Rancho Redondo 2180-2200 m., Dodge 5158 

Collybia or n. gen.

Pileus $4 \mathrm{~cm} .$, convex to expanded, striate margin; disc pecan brown shading to buff pink; margin white Gills white, narrow, crowded, free Stipe cartilaginous, white above, buff pink to ochraceous buff below, $6 \mathrm{~cm}$. $x$ 2mm.; when mature, young stipe shorter and tapering upward

Caespitose on leaf mould

Costa Rica, San Jose prov, canon of $R$. Uruca below Sta. Ana $9-8-36$ D.dge. Goerger 


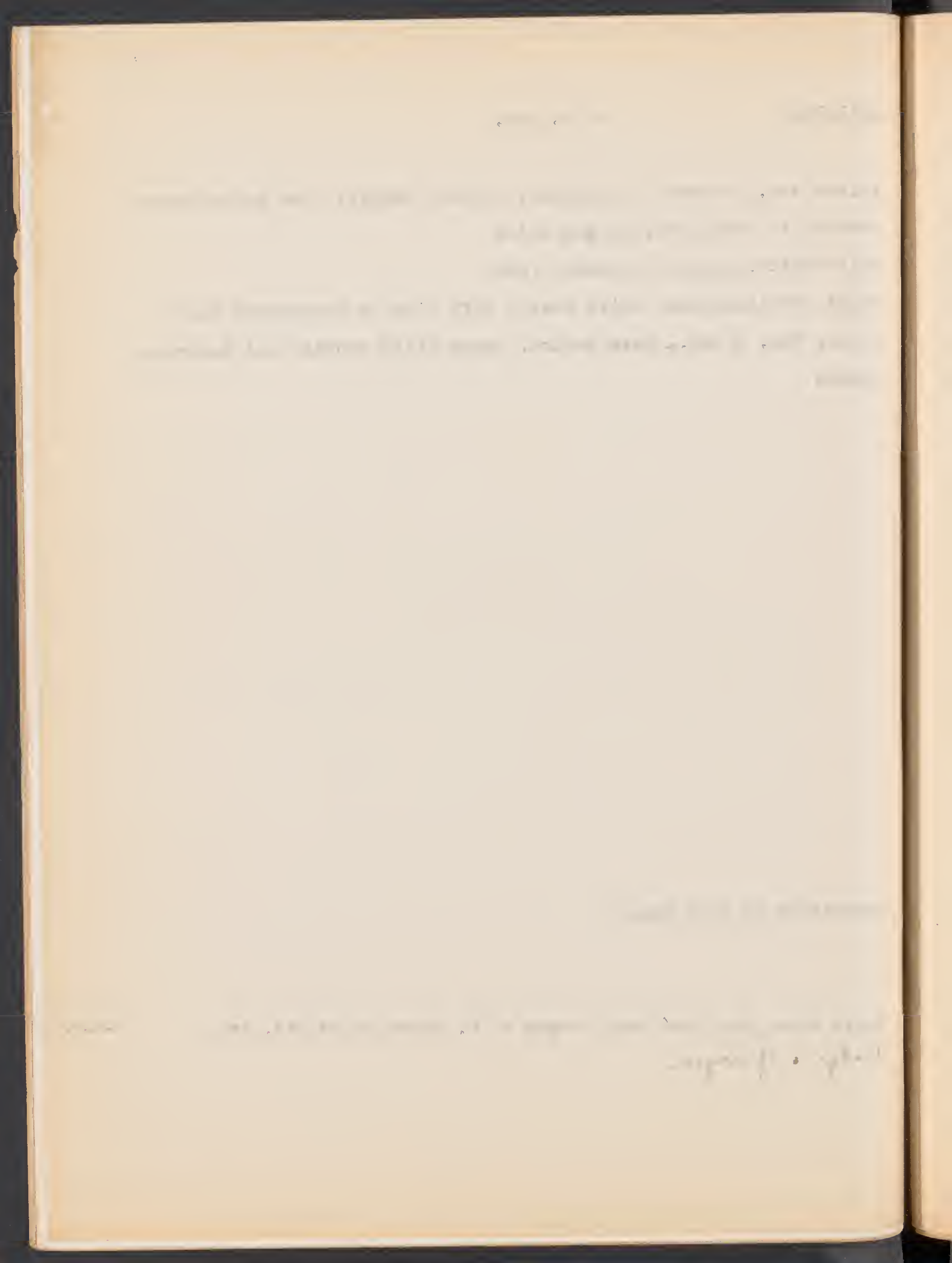


Collybia

New Genus, free gills. mycena?

Stipe cartilaginous, stufiea, f'lattened, $4 \mathrm{~cm}$. Iong \# $5 \mathrm{~mm}$. white.

Mossy $\log$

Specimens examinea:

Costa Rica, San Jose, Rancho Redondo, Alt. 2500, Dodge \& Thomas 5261 
1.

no

st

$\mathrm{ap}$

Ij 
Collybia but ש̇ills free

Pileus dresden brown disc mumm brown, campanulate, $2 \mathrm{~cm}$ in diam. $1.2 \mathrm{~cm}$ tall, innate fibrillose margin narrovly and very slightly striate, not sulcate; gills free, close white narrow

stipe cartilaginous compressed, splitting hygrophanous, brown scaly at apex, $4.5 \mathrm{~cm} \times 2 \mathrm{~mm}$, white

Palm forest

Limon, Siquirres, Castilla, $20 \mathrm{~m}$, Dodge \& Goerger 

Cropicotus nosp.

Pilcus up to srm. surface iensely fibrous, scaly, mas lacerate, extending beyond ilis, betreen ochraceous timy w buclutioun bromn Gilis concolorous on referw derlex, broad, ventricose, edce eroced or prearinc so fron renains of veil, adnete, subsinuate

Stip Iatera, $0.3 \mathrm{~cm}, x$ Imn, fibrous, to entose, Ijonter.

on decayins rood in forest along cirebrada

Costa rica, San José prov, Guarbillos, $2300 \mathrm{~m}$. $7-10-36$

Dodge i Coerger 


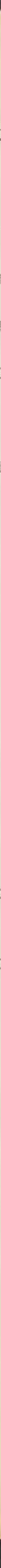


Crepidotus

Mileus up to $3 \times 6$ cno, Minidiate "conic" becoung flatte ed, plocoose, russet, tomentose which findly shows as tpyranidn scalos, noist, buctioom brown marcin mooth orisped mace

Gills ventricose, decurrent, wite becoming wood brom

on Irealyntixs

Costa, Rica, San José provo, Guayabilios

Dodge \& Goerger 


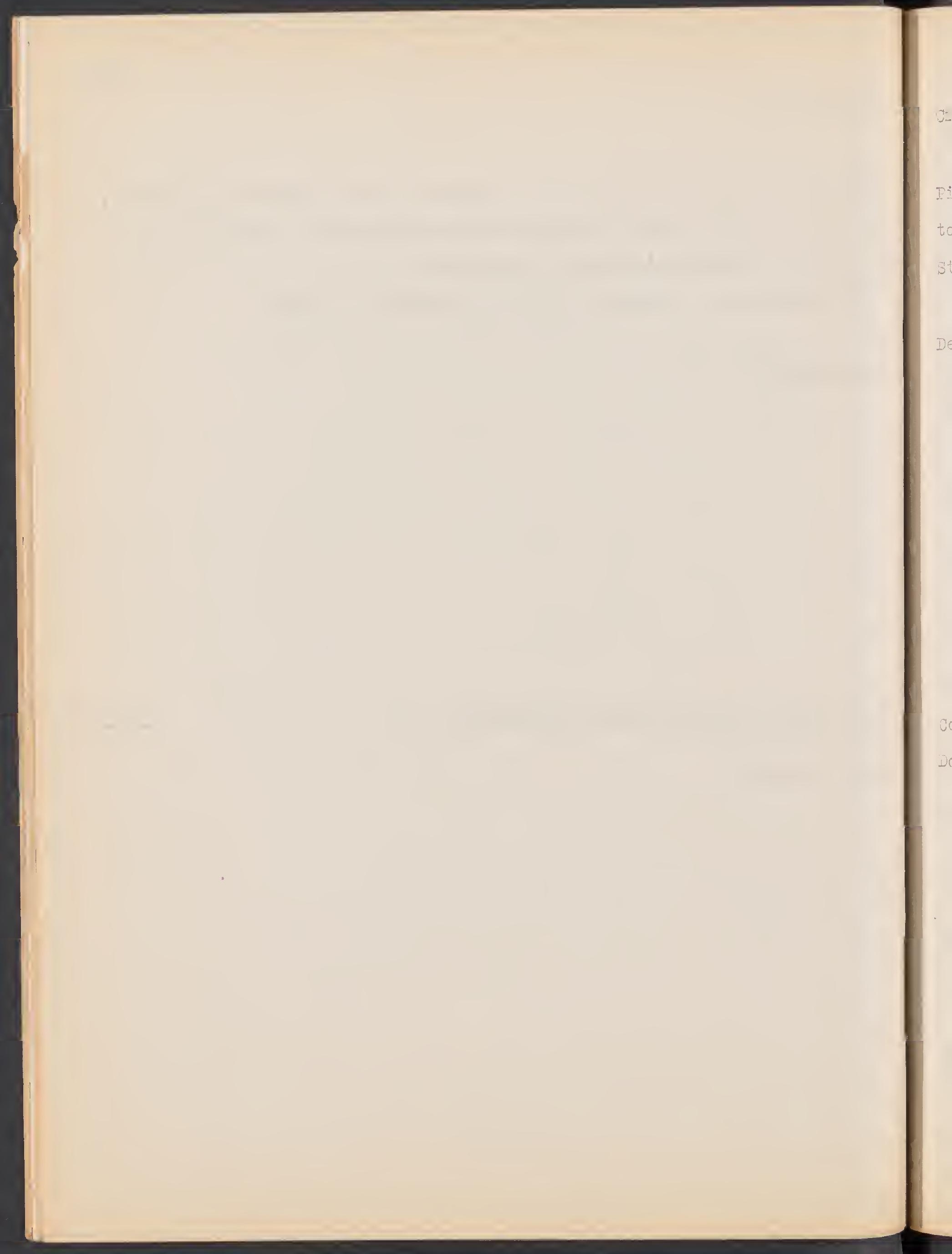


$y_{m}$ epidotus

Iileus derrinte, $.5 \times 1.5$, lensely stricose vith unsset hnims ospecir.IIy torerds marin

Stije lateral, gills decurrent, ventricose, wite to fles? pin?.

Decajing bart on Ioj alon weinad?

Costa lica, san José prov, , tancino Redondo, $2250 \mathrm{~m}$, 


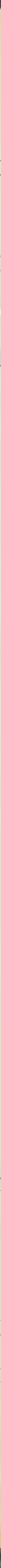


Gaviotus

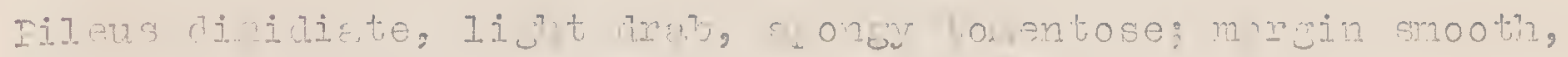

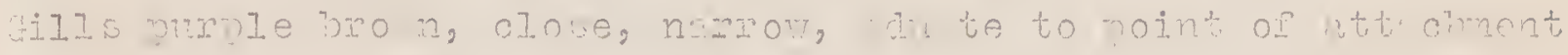

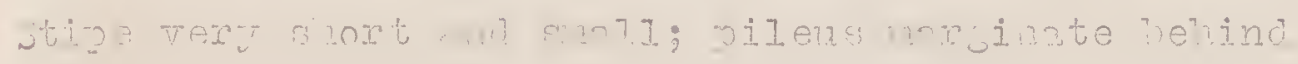

On dicer int iteliooni:

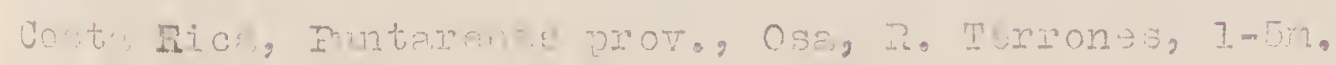




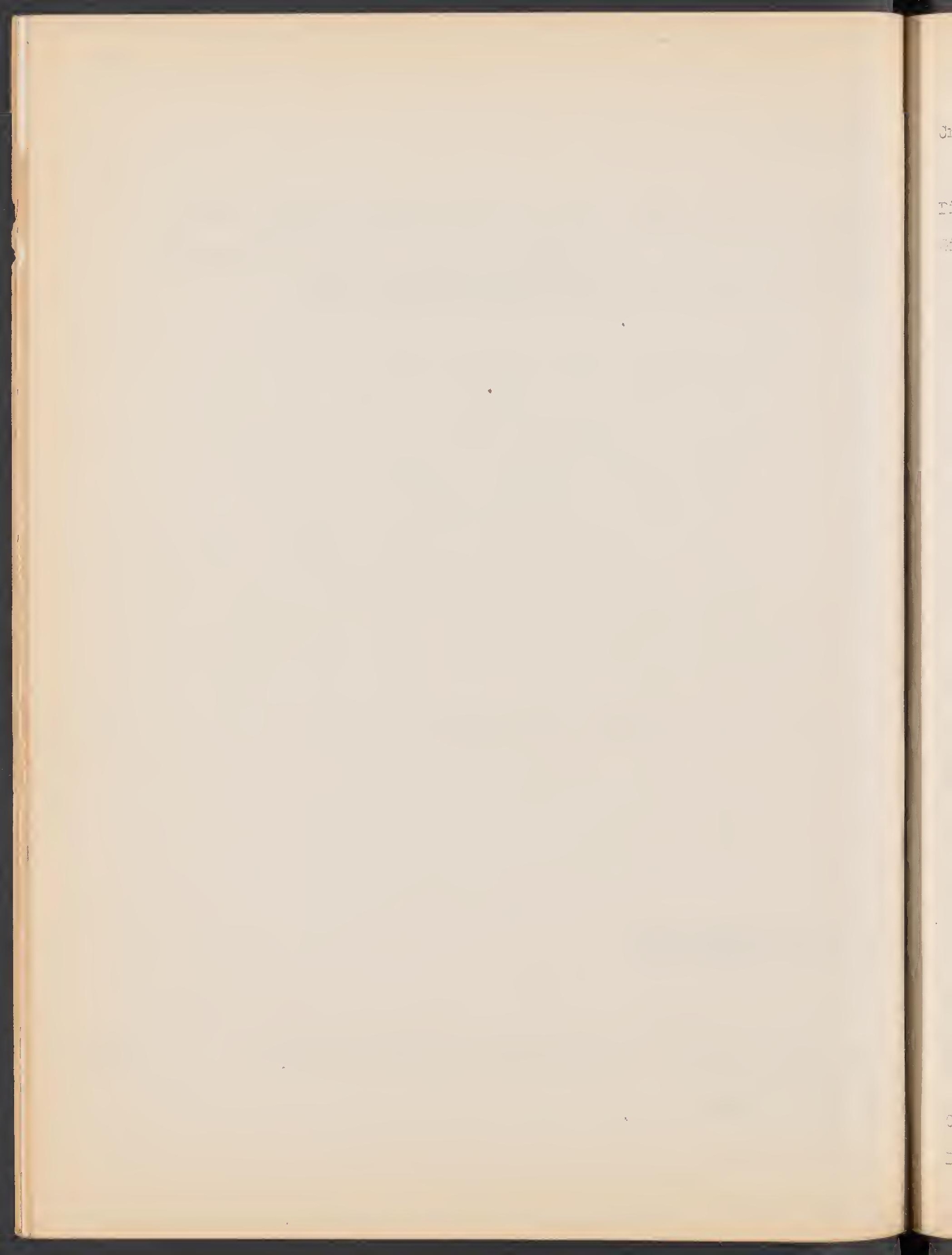


$j=: i c o t, y$

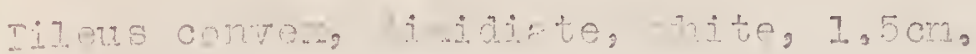

iII a bro n, subitatint, bro: ct

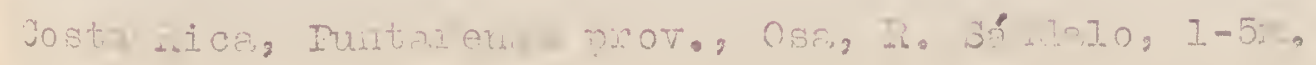





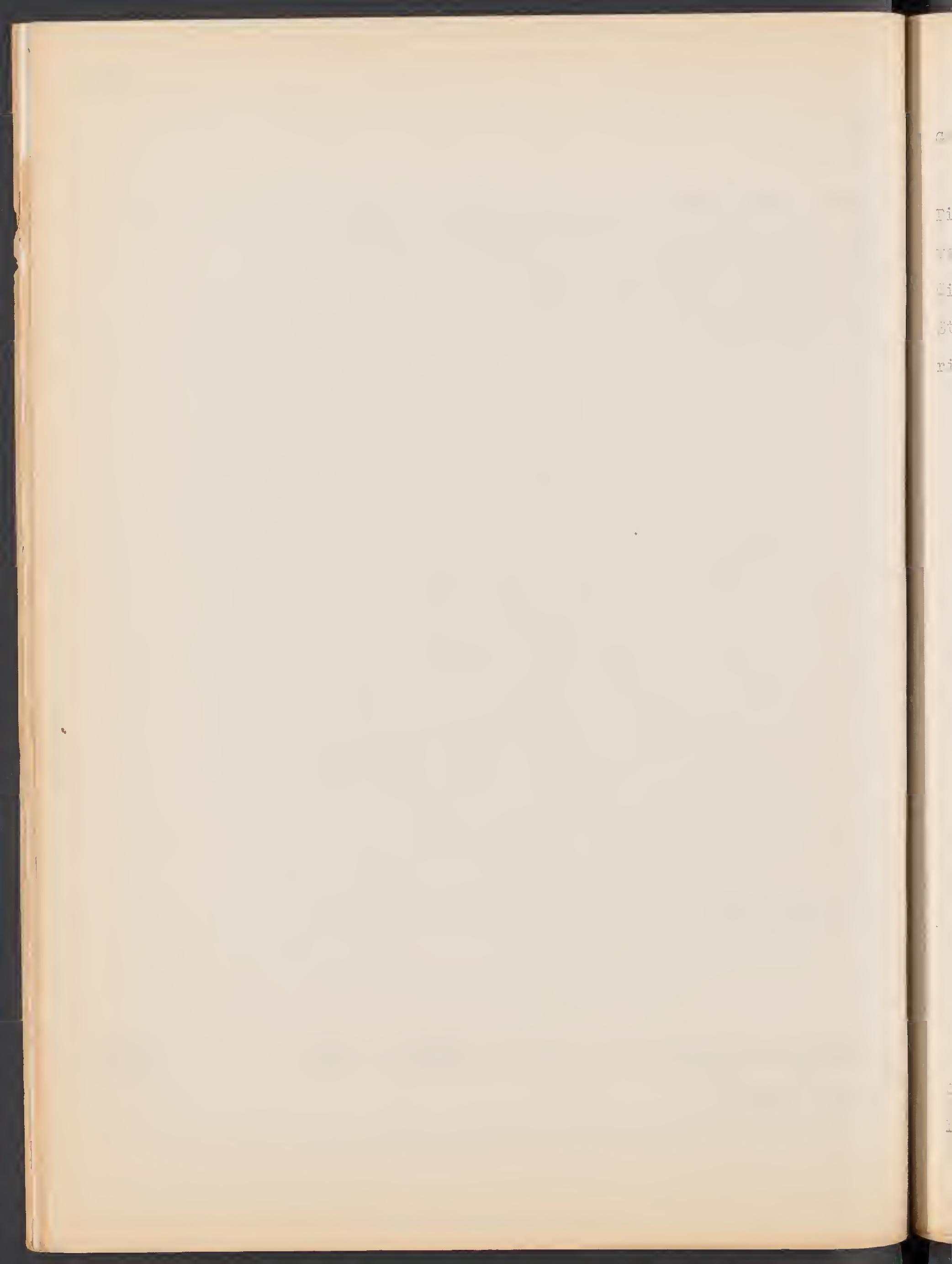


$\therefore-L^{2}$

$0=n \cdot$, on.

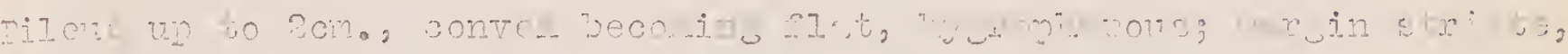
thin, s looth, oimmon to in? ist nuf

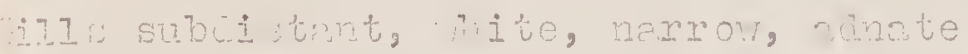

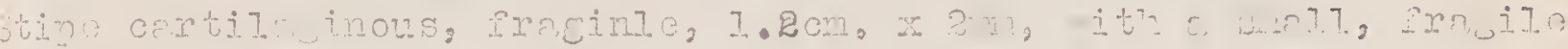
nin, 2 in

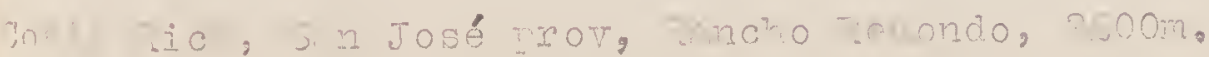




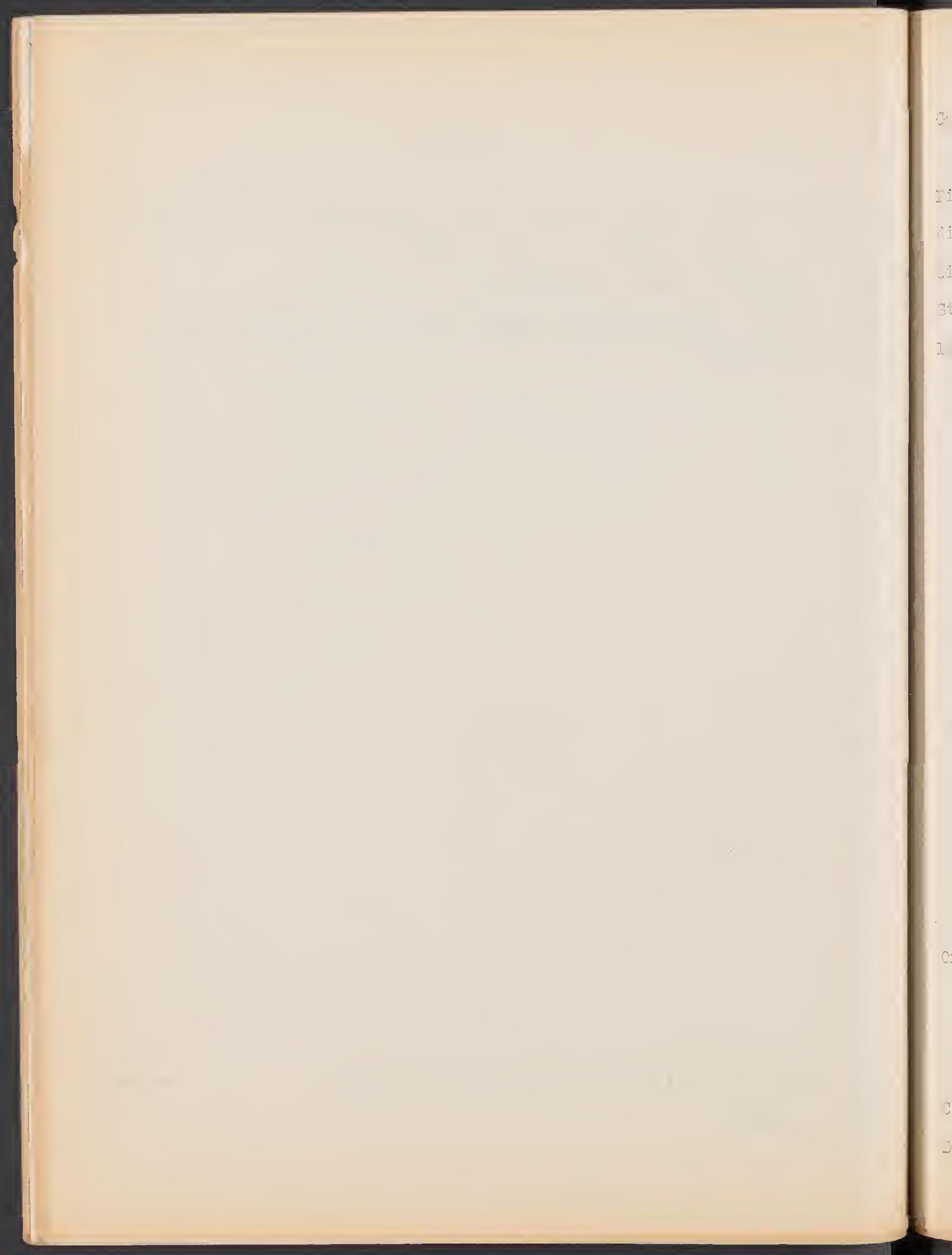


C. Iere

"YCe? yXincions?

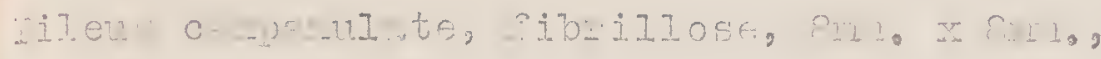

Bnt?

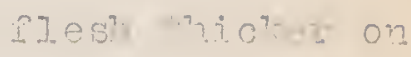

isc, hite

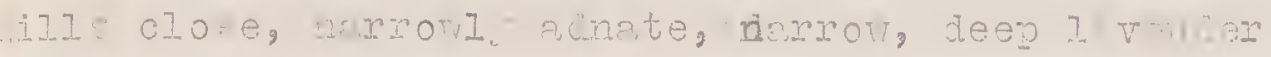

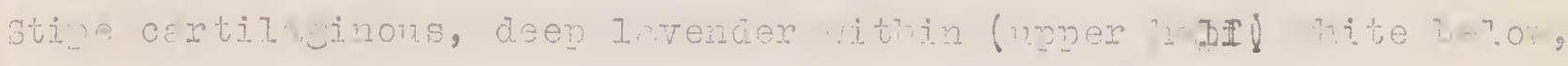

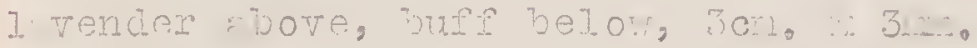

Cx. $\quad 0: 5 y+b \pi, n k$

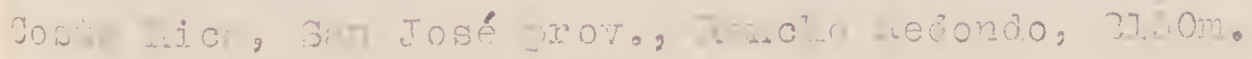




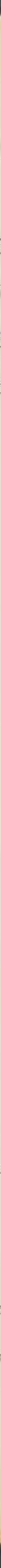


175

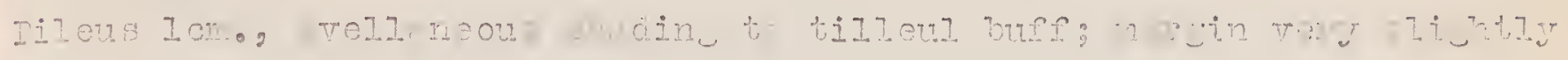
witis to thin

Wills close, nerrow, ainate, aretimneous

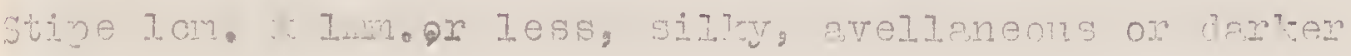

$\varepsilon$

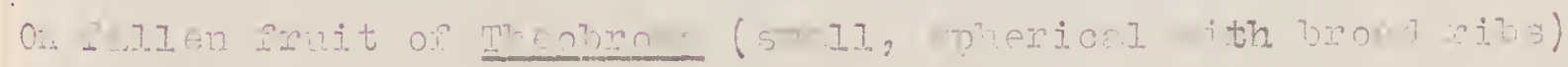

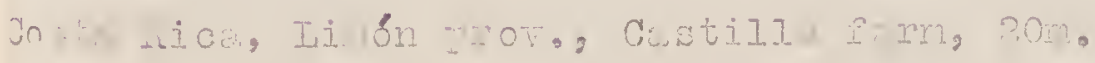

$7-25-30$

Whise w Goerger 


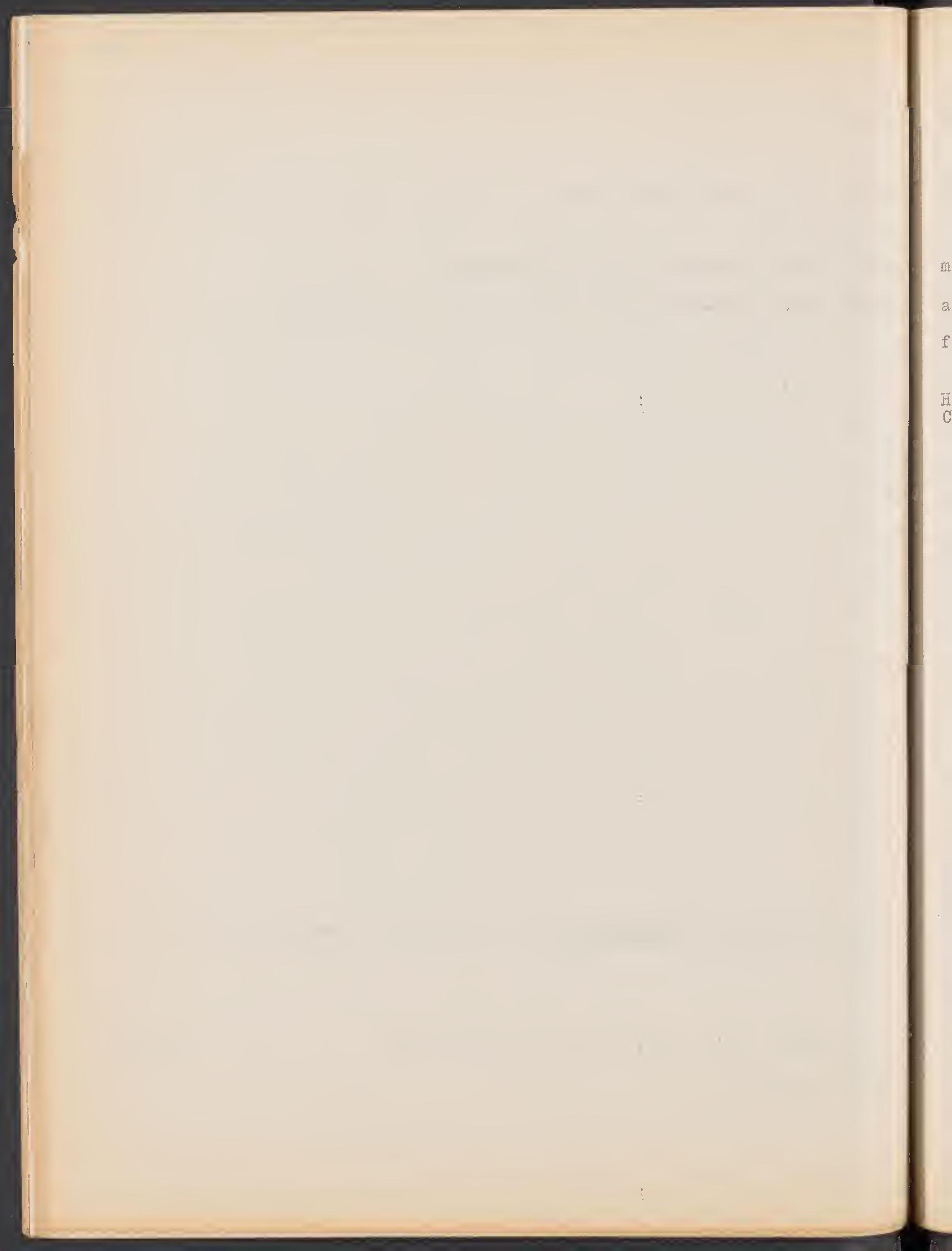




\section{Galera}

Pileus hemispheric to campanulate, $1.5 \mathrm{~cm}$ disc prouts brown, margin ochraceous tawny, $1 \mathrm{~cm}$ tall, margin striate; gills ochraceous adnate, close, broad stipe $7 \mathrm{~cm} \times 2 \mathrm{~mm}$, concolor or Iighter, cartilaginous fragile, smooth

Heredia

Chahuites, $1600 \mathrm{~m}$., Goerger 


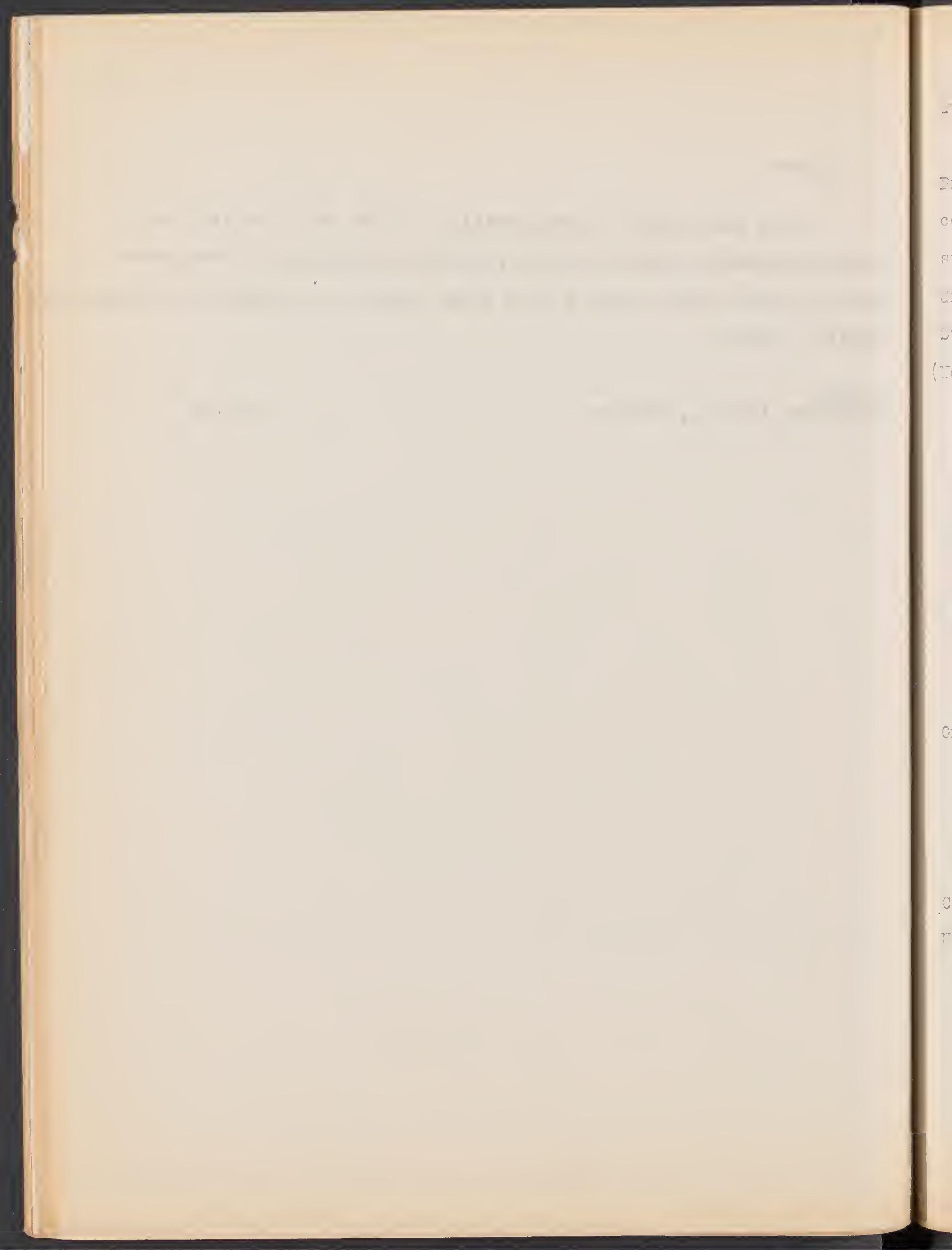


$-\pi$.

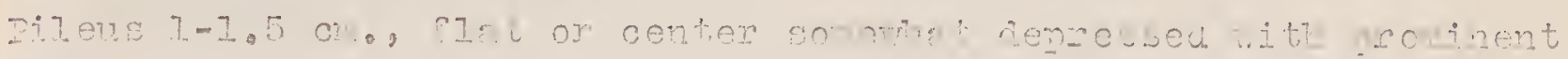

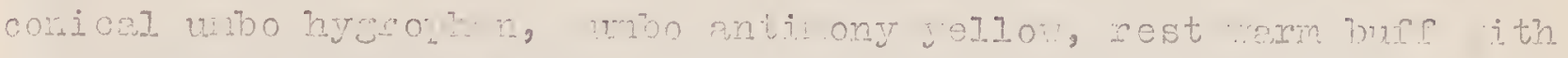
strini,e ochroceous tany" presin.

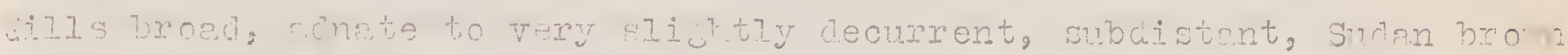

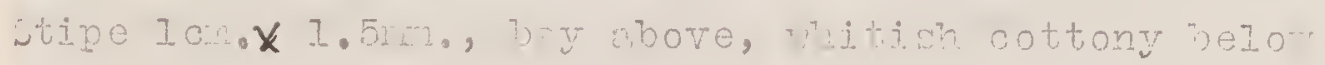

(ronnc specinens in ?.juing inuid)

on sern roots.

Conte -iop, Sen José pror,, San Fedro de Pontes de ocan

Truman c Goerger 


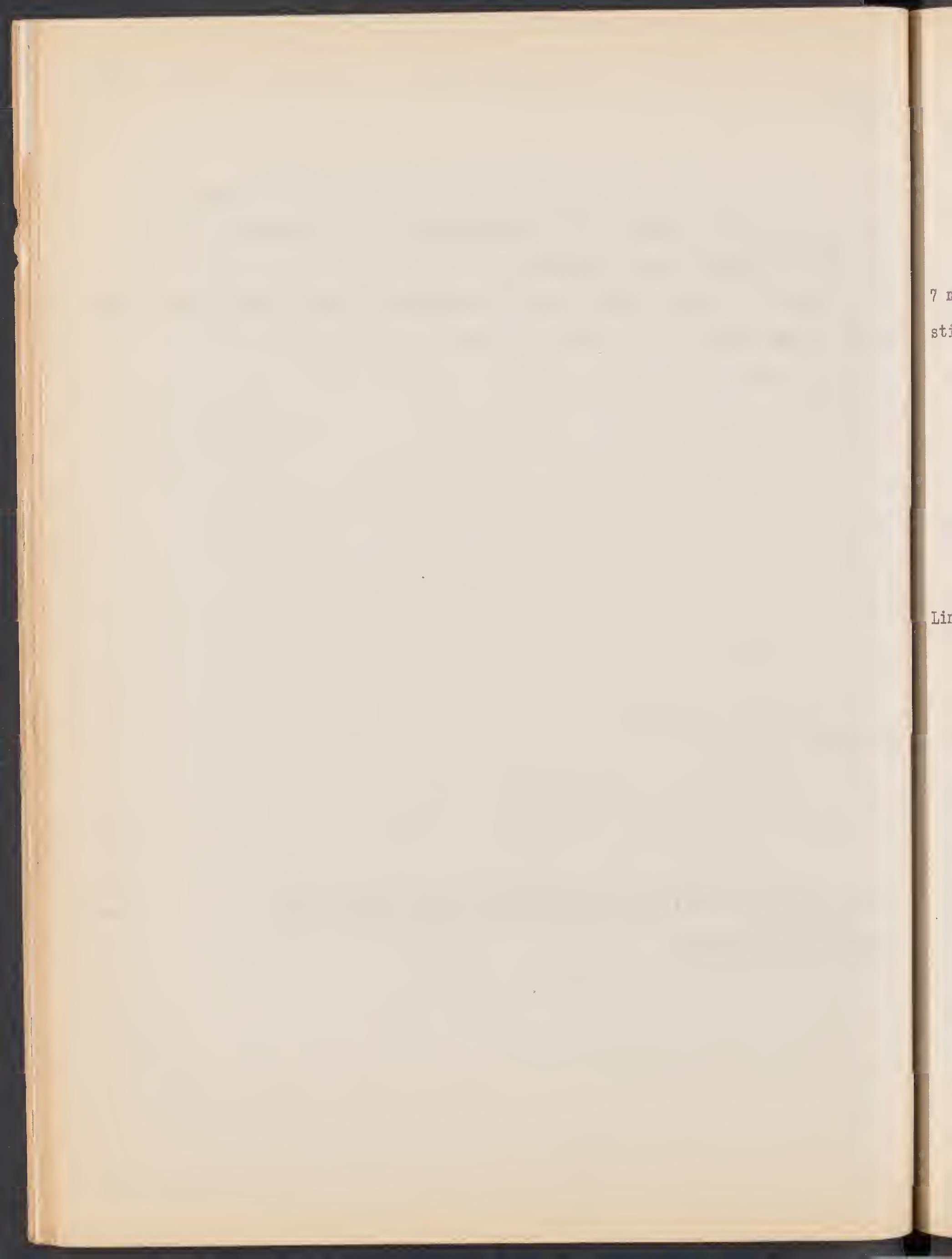


Geopetalum copulatum ??

Crepidopus

Pileus cuneate, $4 \mathrm{~cm} \times 4 \mathrm{~cm}$, crisped finally splitting, lobes about 7 mm broad, white, margins striate, gills broad, close, decurrent white stipe very short, $5 \mathrm{~mm}$ at point of attachment

Limon, Siquirres, Castilla, July 23, Dodge \& Goerger 


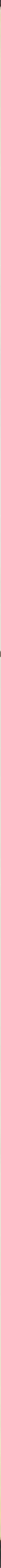


Geopetalum

Pileus flabelliform, $2 \mathrm{~cm}$, smooth, white, hygrophanous, maxgin striate, lobes 3 x $5 \mathrm{~mm}$ long; gills, decurrent along point of a.tachment which is $5 \mathrm{~mm}$ long and wide

Limon, Siquirres, Castilla, Dodge \& Goerger $7-25-36$ 

Hygrophorus

Pileus umbilicate, convex, $5 \mathrm{~cm}$ in diameter, dragons blood red fading to terra cotta, dry; gills waxy white, subdistant, sinuate a.dna,te, broad; stipe compressed, $5.5 \mathrm{~cm} \times 10-6 \mathrm{~mm}$, light cadmium to lemon chrome, cartilasinous hollow

In soil in forest

P. untarenas, Osa, R. Sándalo, $2 \mathrm{~m}$. Dodge \& Goerger 

Hygroohorus

Pileus convex, $2 \mathrm{~cm}$, viscid(?) slightly depressed incenter and Iighter, coral red; gills white, waxy, sinuate adnate with decurrent tooth broad; stipe viscid, $4.5 \mathrm{~cm} \times 3 \mathrm{~mm}$ cartilaginous light cadmium, semipelluc id

Puntarenas, Osa, R. Sándalo, $2 \mathrm{~m}$., Dodge \& Goerger $8-27-36$ 

Iygrophorus

Pileus viscid, flat, margins very irregularly elevated, white, rest coral pink; gills thick waxy, edges eroded broad, subdistant, white; stipe cartilaginous semipellucid coral pink, $3 \mathrm{~cm}$ with thickening suggesting a ring about $1 \mathrm{~cm}$ below apex, 4-5 mm tapering domward 
Hygrophorus

Pileus $3 \mathrm{cr}$, expanded to Blat, disc eventually lacerate, viscid margin slightly striate, Corinthian red shading to Corinthian pink; gills thick, white, broad, close,adnexed; stipe pellucid above cortina, Corinthian pink below white cottony becoming vinaceous tawny where handed. wholly viscid, $4.5 \mathrm{~cm} \times 5 \mathrm{~mm}$

Cespitose in soil

Puntarenas, Osa, R. Séndalo, 2 m., Dodge \& Goerger 


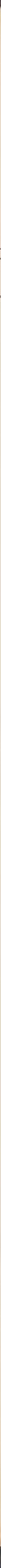


Hygrophorus

Pileus convex, viscid, $2 \mathrm{~cm}$ deep vinaceous, lighter at margin; gills waxy white, broad close apparently free[but somewhat eaten] stipe white pruinose above, viscid below where handled, concolor, $3 \mathrm{~cm} . \times 4 \mathrm{~mm}$.

Puntarenas, 0sa, R. Sándalo, $2 \mathrm{~m}$, Dodge \& Goerger 



\section{Hygrophorus}

Pileus 1.7 cm., very viscid, margin thin almost white, deep chrome striate to disc. which is grenadine red; gills white; stipe viscid to apex, cartilaginous shining $4 \mathrm{~cm} \times 2 \mathrm{~mm}$, grenadine red above shadingto deep chrome

Funtarenas, Osa, R. Sánda,10, $2 \mathrm{~m}$., Dodge \& Goerger 


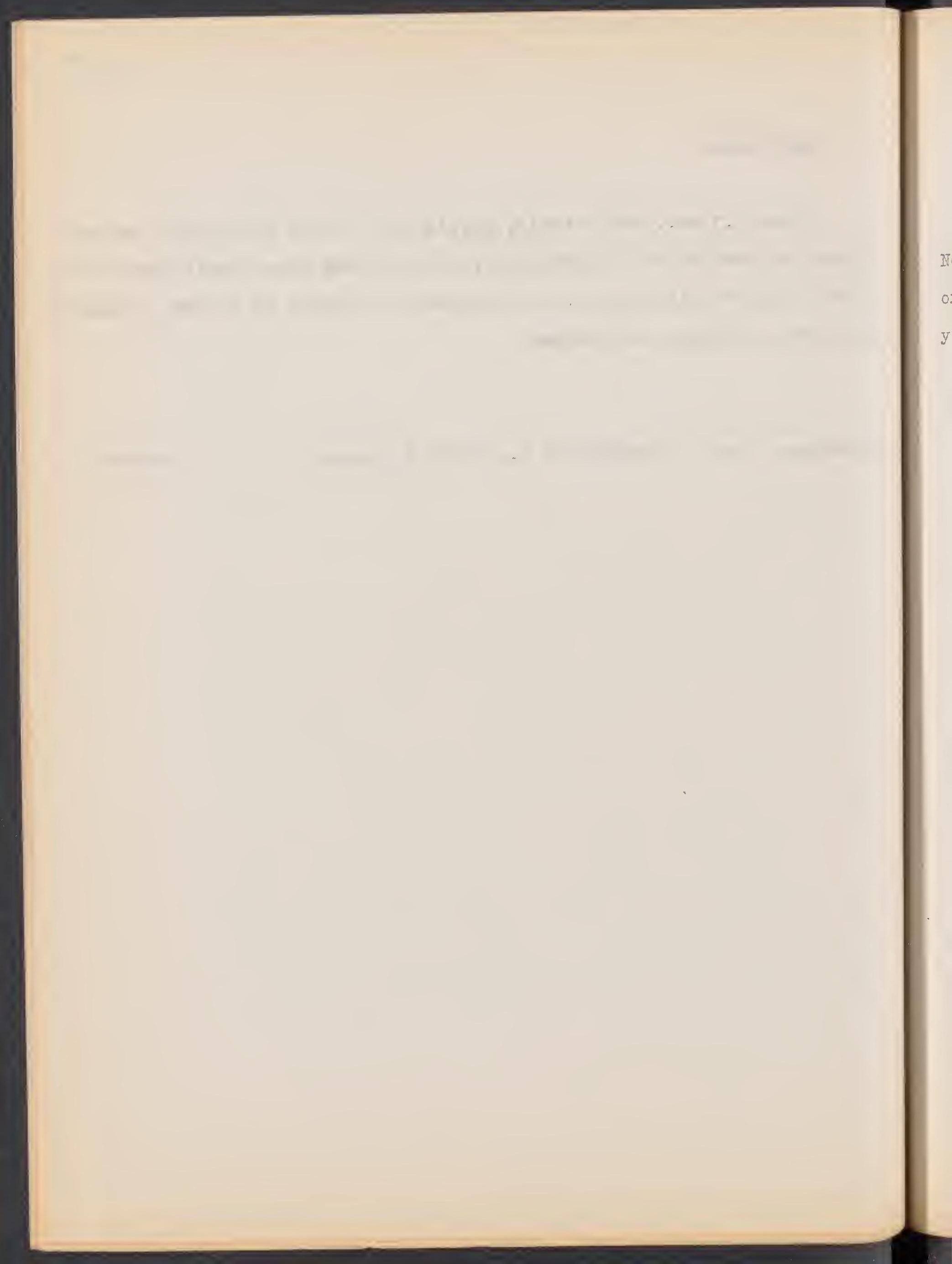




\section{Hygrophorus}

Pileus viscid, $1.5 \mathrm{~cm} .$, convex, umbilicate, striate to disc. Hopal red, margins white or nearly so; gills subdistant, waxy light orange buff, adnate; stipe cartilaginous, viscid, $3 \mathrm{~cm} \times 2 \mathrm{~mm}$, cadmium yellow

Puntarenas,0sa,R. Sándalo, 2 m., Dodge \& Goerger 
Fygrophorus

Pileus convex, $1.3 \mathrm{~cm}$, slightly spongy tomentose, scarlet red, gills thick, waxy, white adnate with decurrent tooth; stipe $4 \mathrm{~cm} \times 33 \mathrm{~mm}$ fleshy, silky, cadmium zellow or lighter

Puntarenas, 0sa, R. Sandalo, $2 \mathrm{~m}$., Dodge \& Goerger $8-24-36$ 

Hygrophorus

Pileus convex $1.5 \mathrm{~cm}$ in diam, viscid, spectrum red, margin undulate more or less lacerate, not striate; gills white, waxy, broad, subdistant, slightly decurrent, edges eroded; stipe cartilaginous, viscid, scarlet, $4 \mathrm{~cm} \times 2 \mathrm{~mm}$ 
$c e$
$d i$
$1 a$

ad

$\mathrm{Pu}$ 


\section{Hygrophorus}

Pileus $3.5 \mathrm{~cm}$, center depressed, margins irregularly elevated, viscid center wax yellow, margins ochraceous orange; gills broad, adnate, subdistant, amber yellow to straw yellow; stipe conor of gills, viscid, cartilaginous, hollow, compressed, semipellucid, $6 \mathrm{~cm} \times 8 \mathrm{~mm}$.

Specimen same locality Aug. 25, pileus $4.5 \mathrm{~cm}$, irregularly expanded, margin very faintly striate, wax yellow to amber yellow, dry; gillssinuate adnate, white; stipe wax yellow, $5 \mathrm{~cm} \times 6 \mathrm{~mm}$,

Puntarenas, Osa, R. Sándalo, 2 m., Dodge \& Goerger 9-1, 8-25, 36 


\section{Hygrophorus}

Pileus convex, slightly umbilicate, I cm, dry, disc ligher color than rest which varies from cadmiun yellow to lemon chrome; gills white, subdistant, slightly decurrent, narrow (fully mature); stipe $2 \mathrm{~cm} \times 3 \mathrm{~mm}$. Iemon chrome

Puntarenas, Osa, R. Sándalo, $2 \mathrm{~m}$., Dodge \& Goerger 


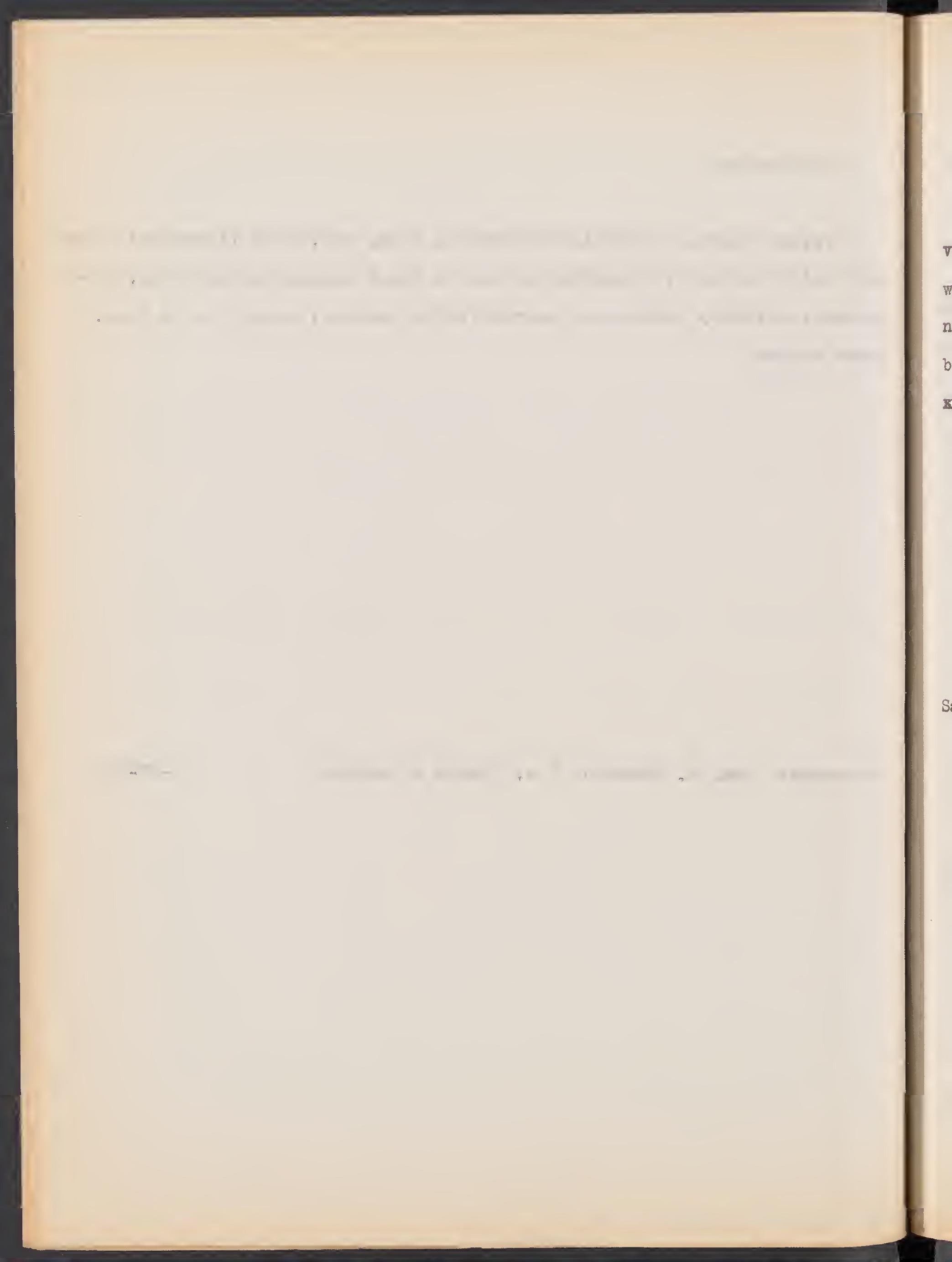




\section{Hygrophorus}

Pileus hemispheric in smallest specimen to expanded in largest, very viscid, scarlet red to scarlet to orange chrome on largest, 5-19 mm; gills white at first, becoming Naples yellow, close, waxy, free or nearly so narrowly ventricose; stipe fragile, scarlet above to light cadmium below, becoming zemon yellow above to white below, moist, innate fibrillose silky, $1-3.5 \mathrm{~cm} \times 1-3 \mathrm{~mm}$.

Under Chusquea

San José, Guayabillos, 2300 m., Dodge \& Goerger $7-7-36$ 

Hygrophorus flammea

bella (cf. spores)

Pileus cadmikum orange fatding to strontian yellow, $2-4.5 \mathrm{~cm}$, deeply umbilicate, irregularly convex; gills subdistant, waxy, broad, concolor with stipe or paler, decurrent; stipe cadmium yellow to strontium yellow $5 \mathrm{~cm} \times 8-5 \mathrm{~mm}$ ta.pering downward, waxy.

Under Chusquea

San José, Grayabillos, $2200 \mathrm{~m}$ Dodge \& Goerger, July 11 


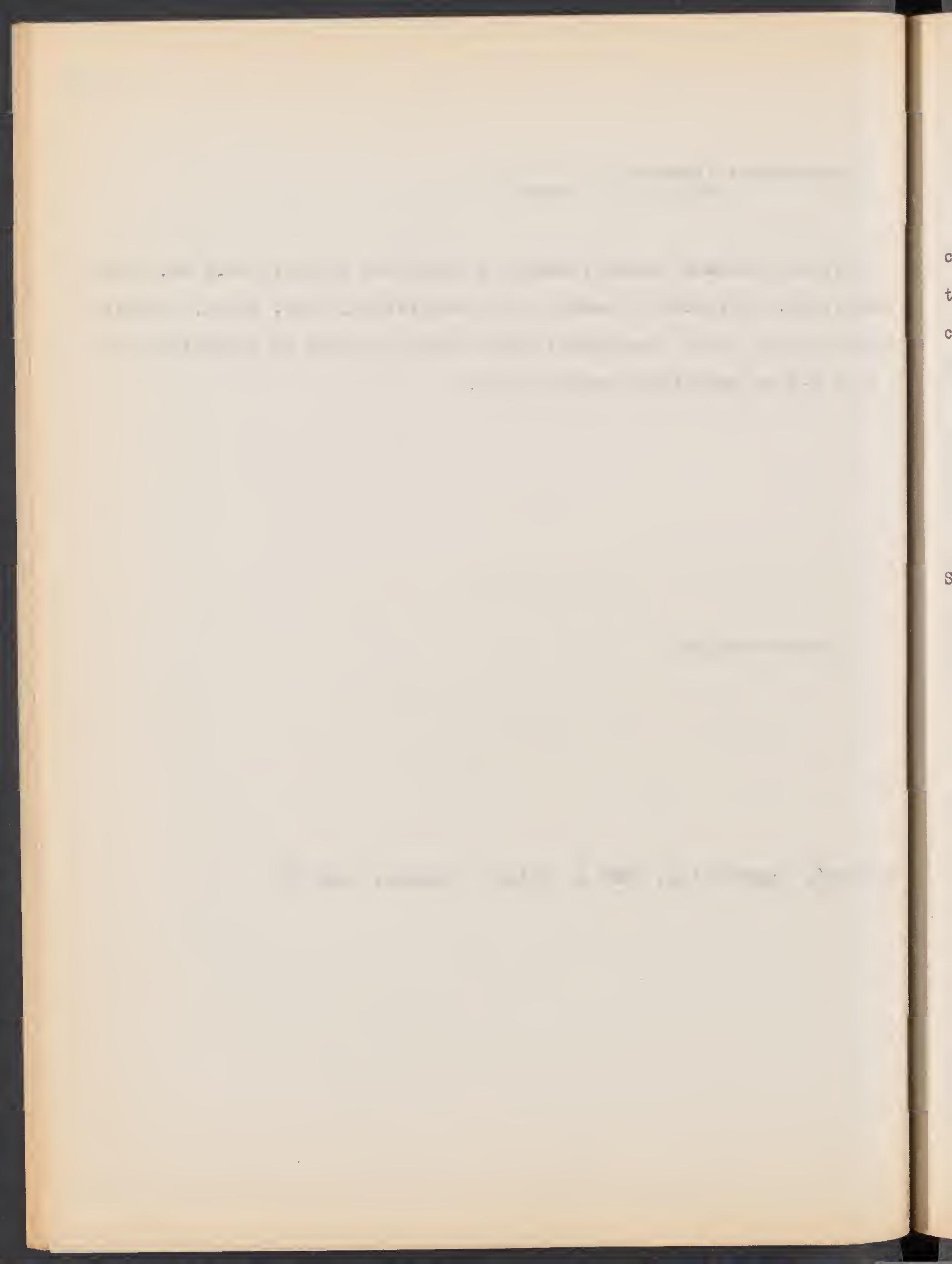




\section{Hygrophorus}

Pileus flat, 5-6 mm., cracked, surface moist, not viscid, smooth, coarsely striate margin when old, hair brown to chaetura drab; gills thick, distant, slightly decurrent, white; stipe $2 \mathrm{~mm}$ wide, $5 \mathrm{am}$ tall concolor, smooth; whole very hygrophanous, no ring

San José, Grayabillos, 2300 m., Dodge \& Goerger 
Hygrophorus

Pileus $1.5 \mathrm{~cm}$, expanded umbilicate, viscid, nopal red, martin striate sulcate, thin, gills peach red, waxy distant, danate; stipe 1.7 cr $\times 3-4 \mathrm{~mm}$, ventricose, mahogany red, viscid

Heredia, Santo Domingo de Sta. Barbara 1500 m., Dodge \& Goerger $8-7-36$ 
Iaccaria

Fileus convex to hemispheric, disc slightly merulioid, marging long striate, dark liviä brown; gills lavender, slightly decurrent, subdistant, broad; stipe concolor above, lighter below, cartilaginous hollow, $4 \mathrm{~cm} \times 3 \mathrm{~mm}$, pruinose above, fibrillose below.

Heredia, Chahuites, $1600 \mathrm{~m} .$, Goerger $9-11-36$ 


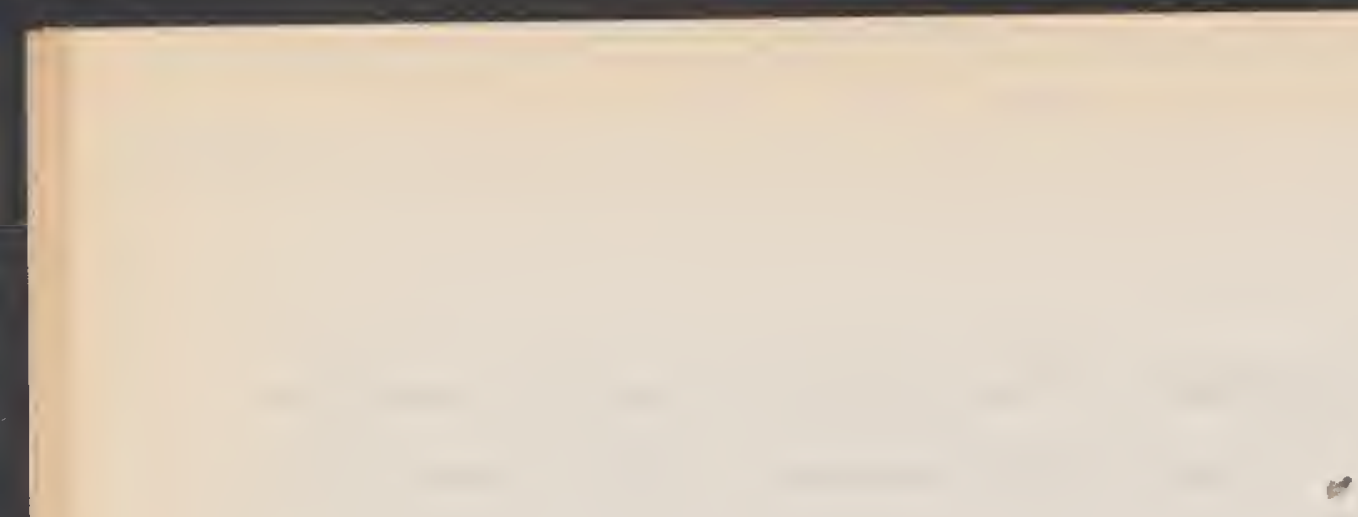


Laccaria

Pileus campanulate, $1.5 \mathrm{~cm} \times 1 \mathrm{~cm}$. hich, margin striate, avellaneous and vinaceous buff; gills adnate to slightly decurrent, subistant, broad white or nearly so; stipe $4 \mathrm{~cm} \times 3 \mathrm{~mm}$, cartilaginous, hollow, concolor 


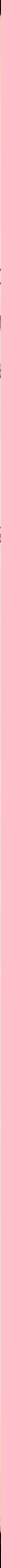


Iaccaria?

Pileus $2.5 \mathrm{~cm}$, convex, sulcate striate over gills; disc and striae Prussian red, rest ochre red; gills very slightly decurrent, subdistant broad, lavender; stipe $4 \mathrm{~cm} \times 2-4 \mathrm{~mm}$ tapering upward, cartilaginous hollow, hygrophanous, innate fibrillose

Heredia, Chahuites, 1600 m., Goerger $9-11-36$ 


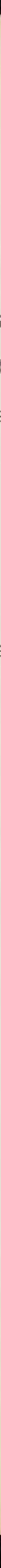


Lepiota?

Pileus $1.5 \mathrm{~cm}$, pyramidal scaly on mars brown disc, appressed scaly over rest, margin appendiculate; gills free, crowded white, ventricose stipe smooth above, cortinate tomentose below, Etruscan red, ligher above, darker below, $1.8 \times 1.5-3 \mathrm{~mm}$ tapering downward.

San José, Santana, cañon of R. Uruca, Dodge \& Goerger $9-8-36$ 
Lepiota

Pileus flat, $2 \mathrm{~cm} .$, disc tawny, breaking up into very small, scatter ed scales on a white ground, margin appendiculate lacerate scaly; gills white, free, ventricose, close; stipe $3 \mathrm{~cm}$, white or nearly so, silky above ring which is erect, fragile, white, slightly floccose tomentose below ring.

Under Chusquea

San José, Santana, cañon of R. Uruca, Dodge \& Goerger 

1.00

$T=40 \ldots:-$

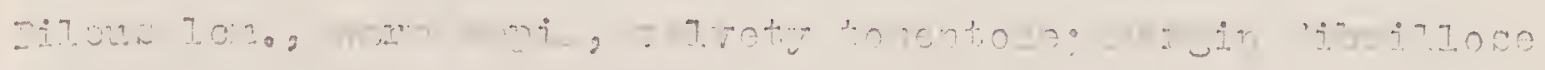

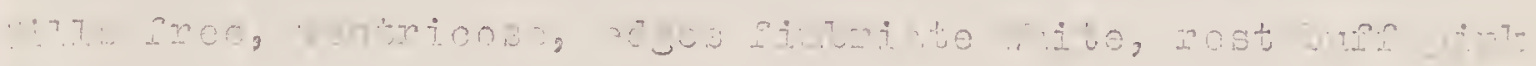

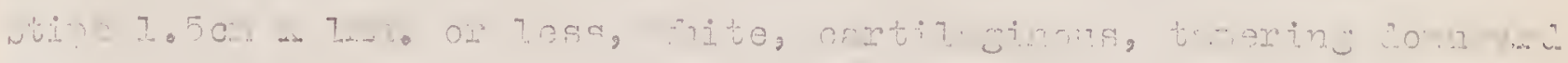

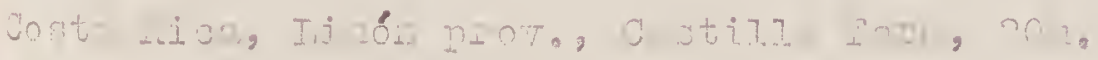

$r=97-36$

onu - Dogr jer 


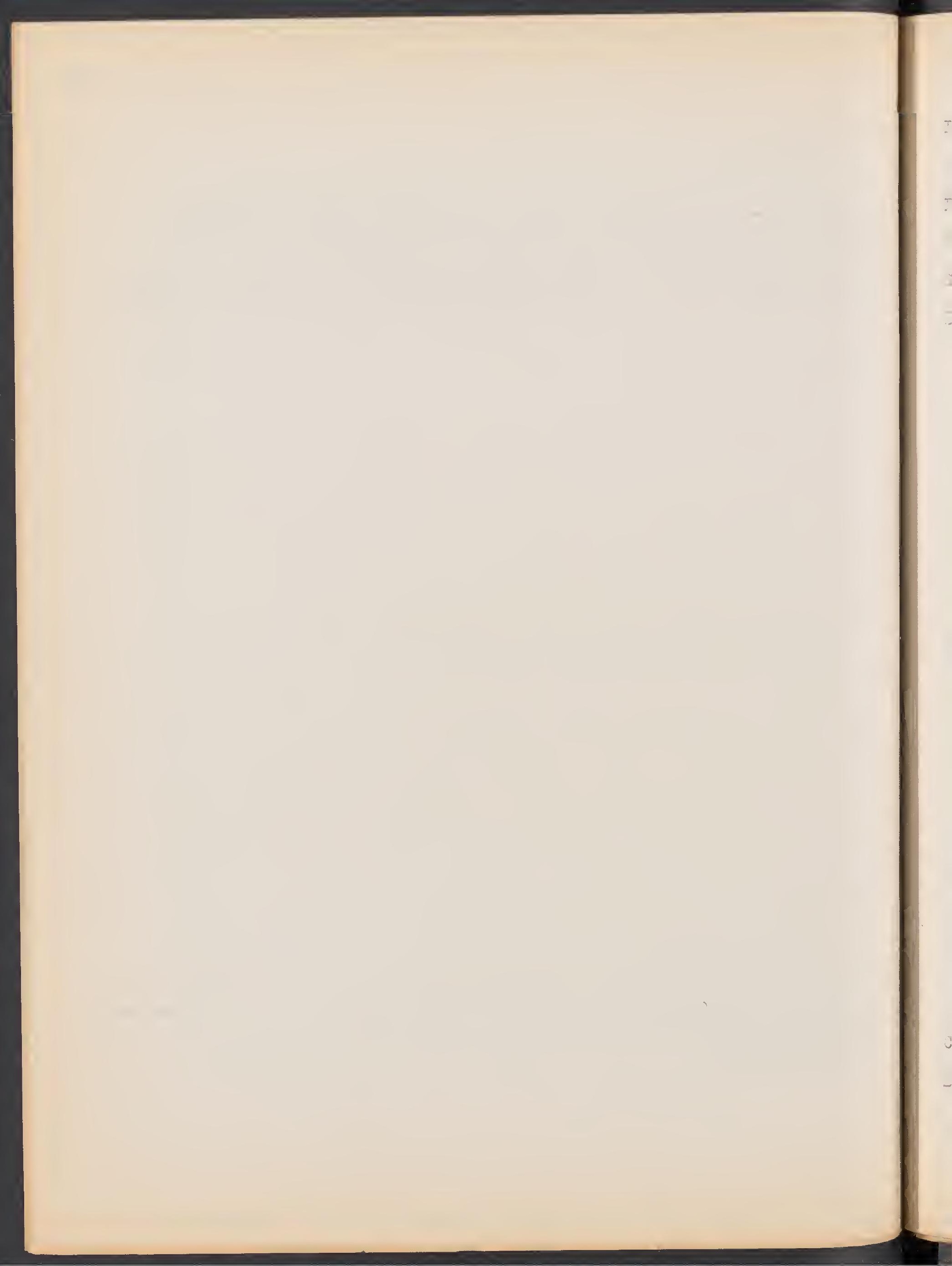





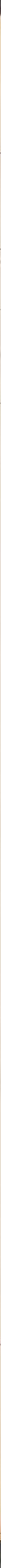


$10 \%$

$+\cdots \vdots-$

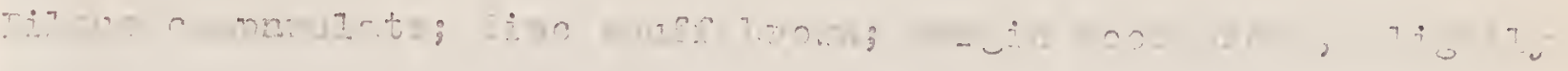

$\therefore 7 \cdot 0$.

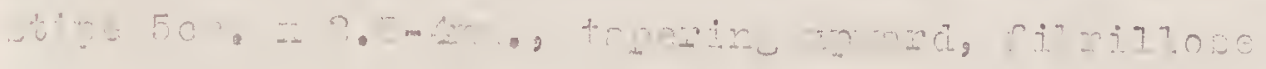

A. w $\sim$ iI?

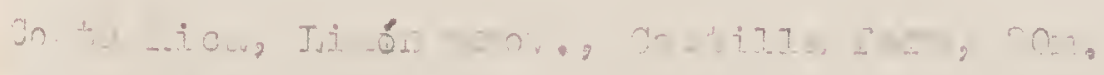

$-430$

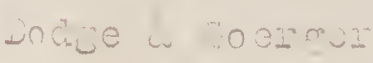




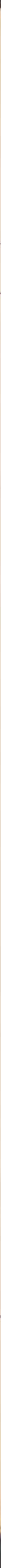


203

Fintion:

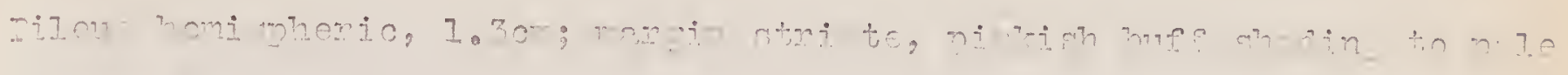

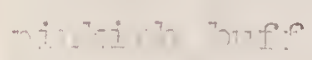

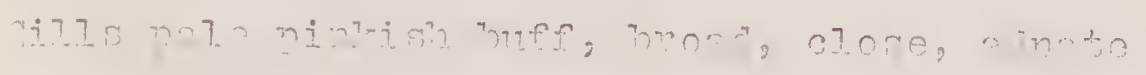

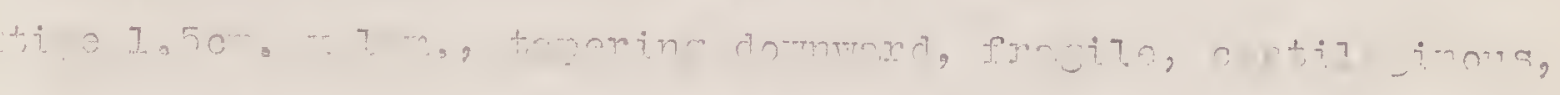

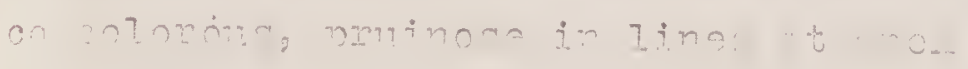




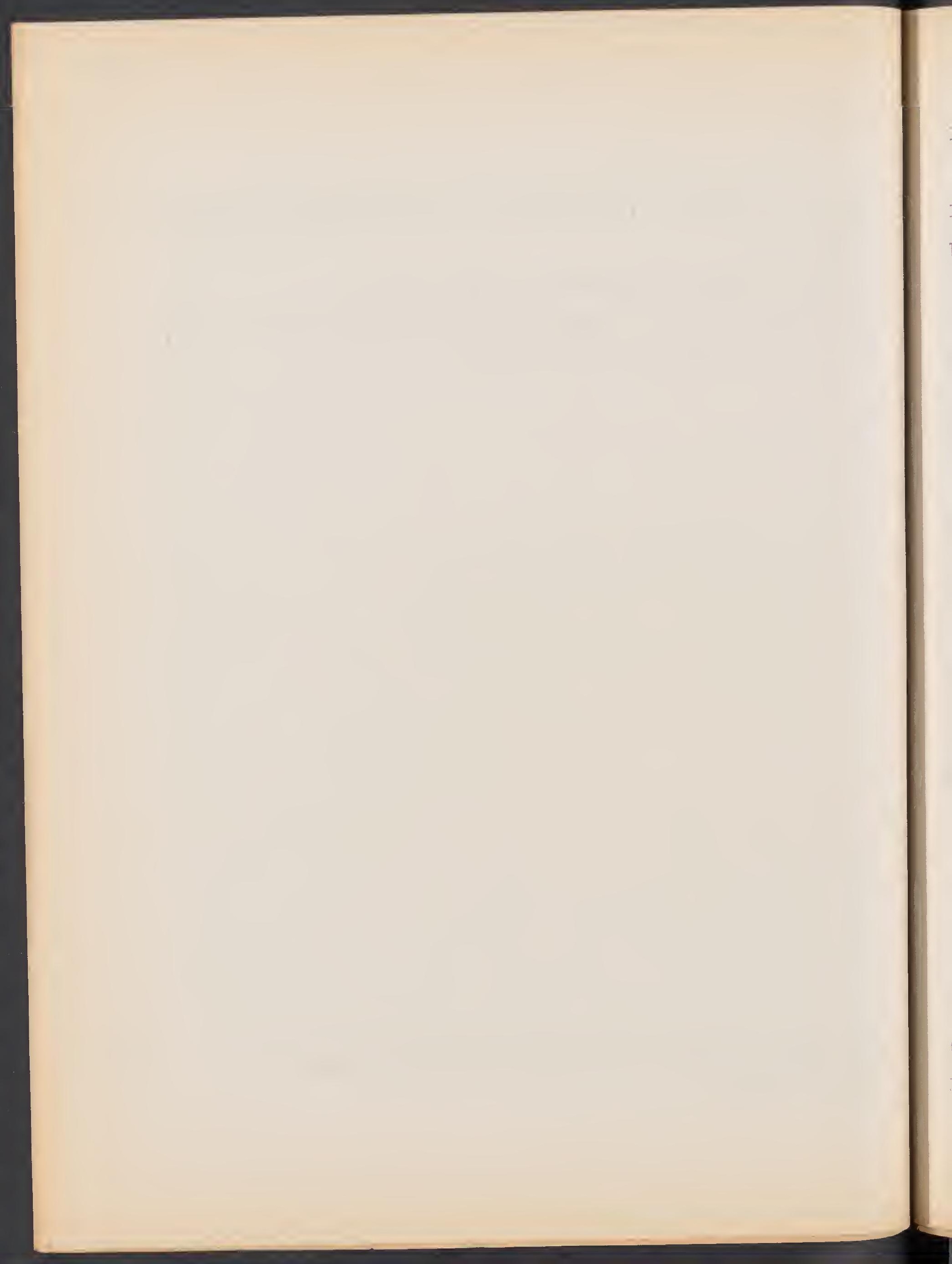


204

Tentoni

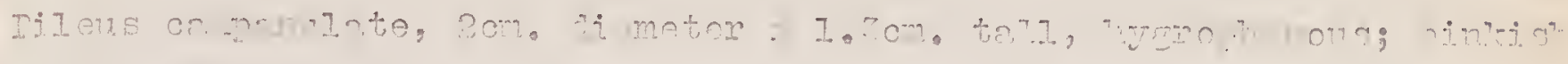

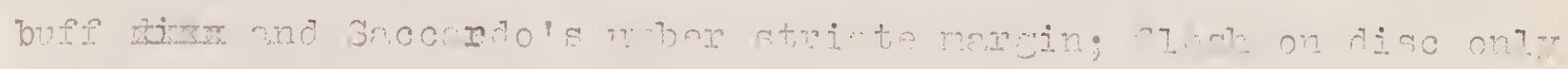

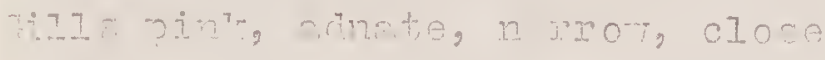

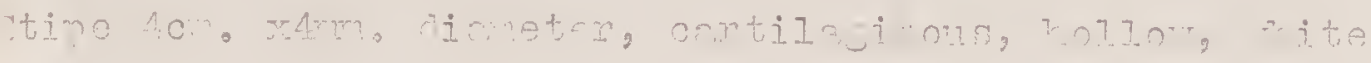

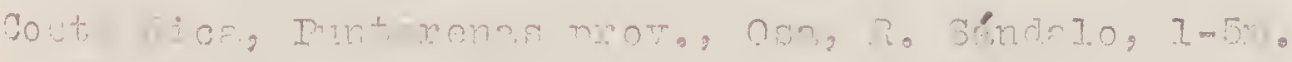

rman-m

Doze Toerser 


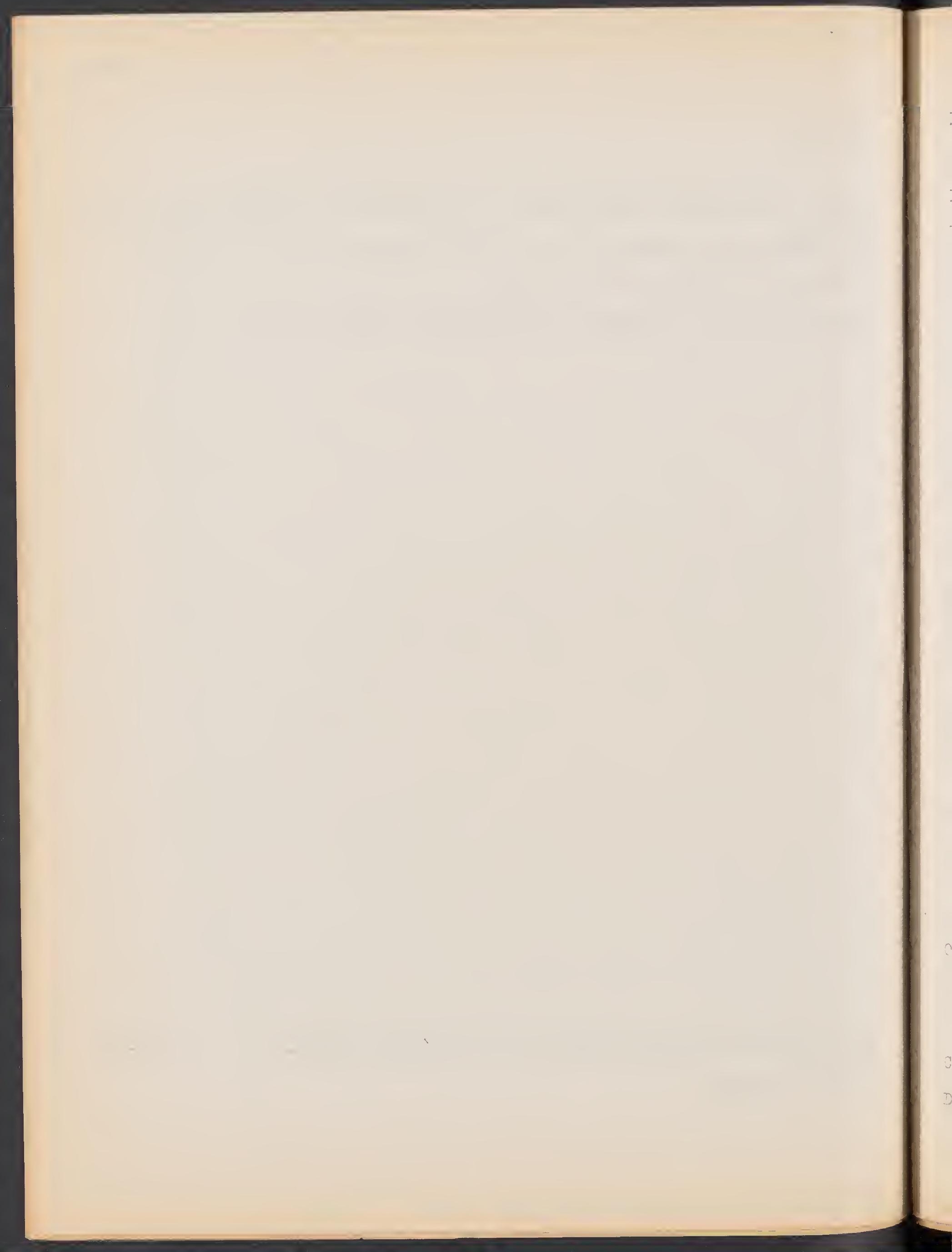





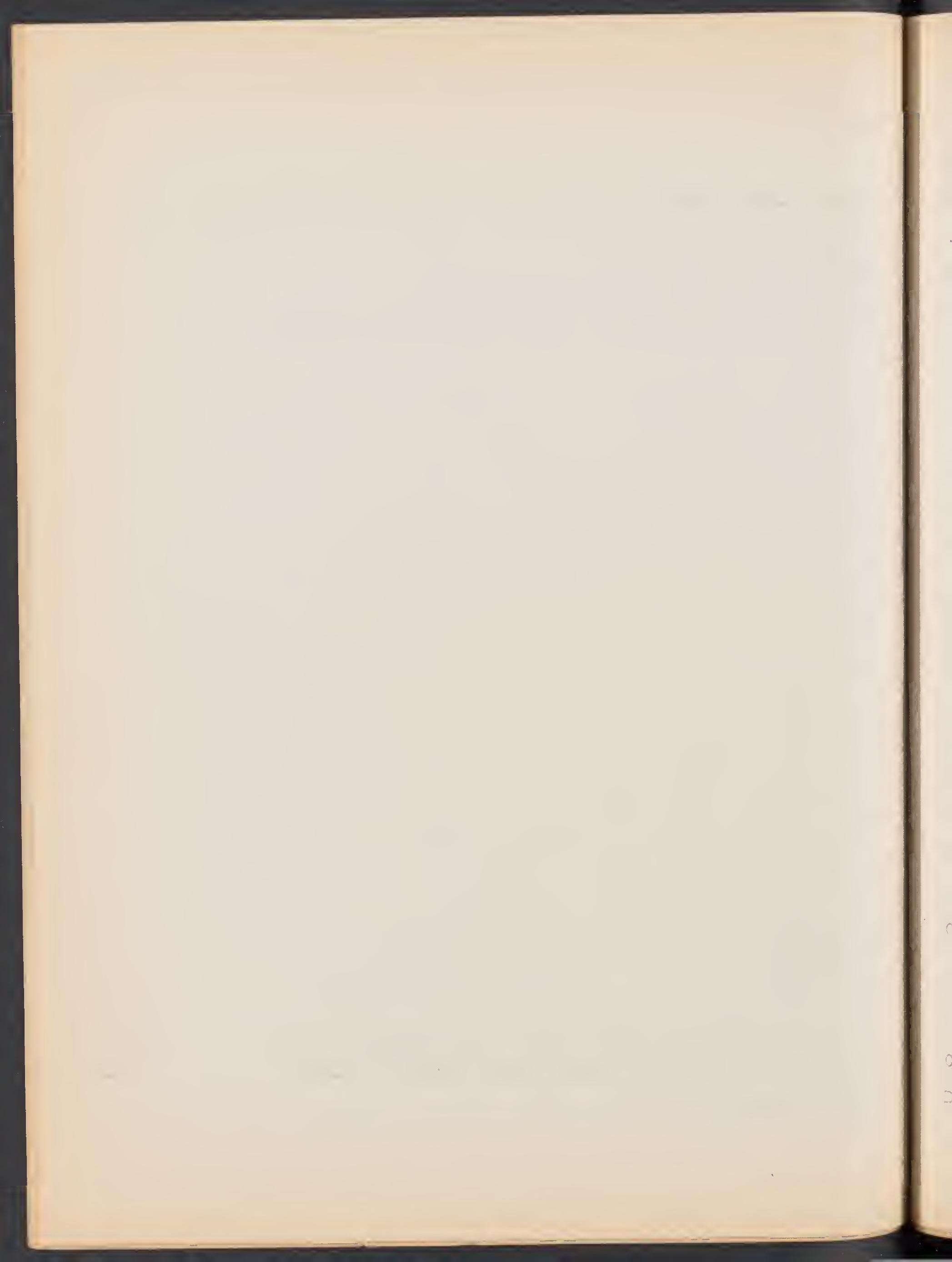


206

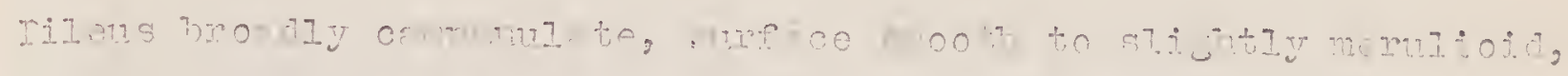

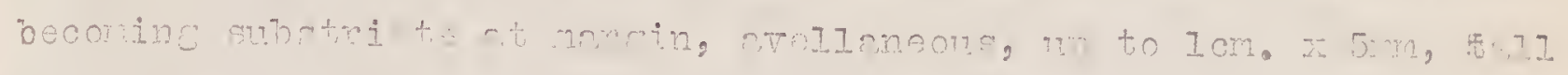

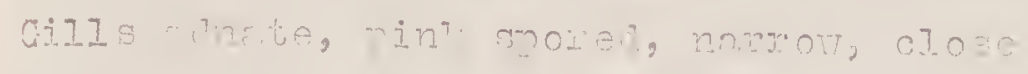

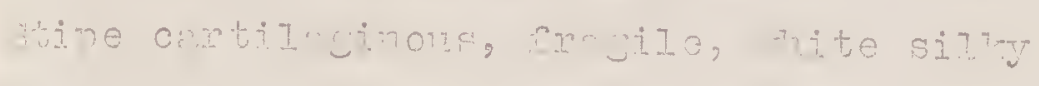

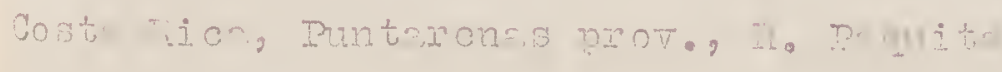

$-1 .-2=6$ Dorde d roeruer 


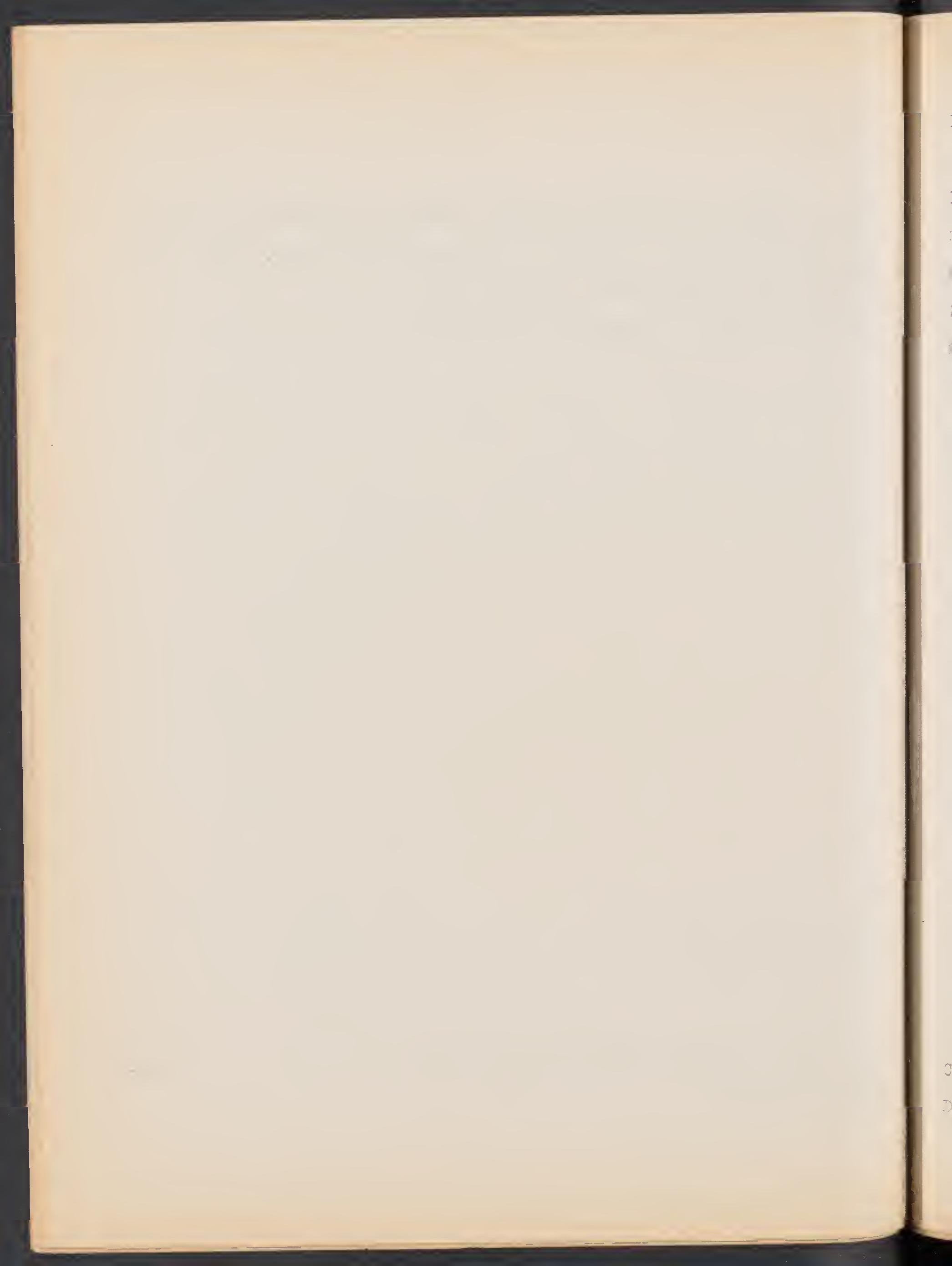





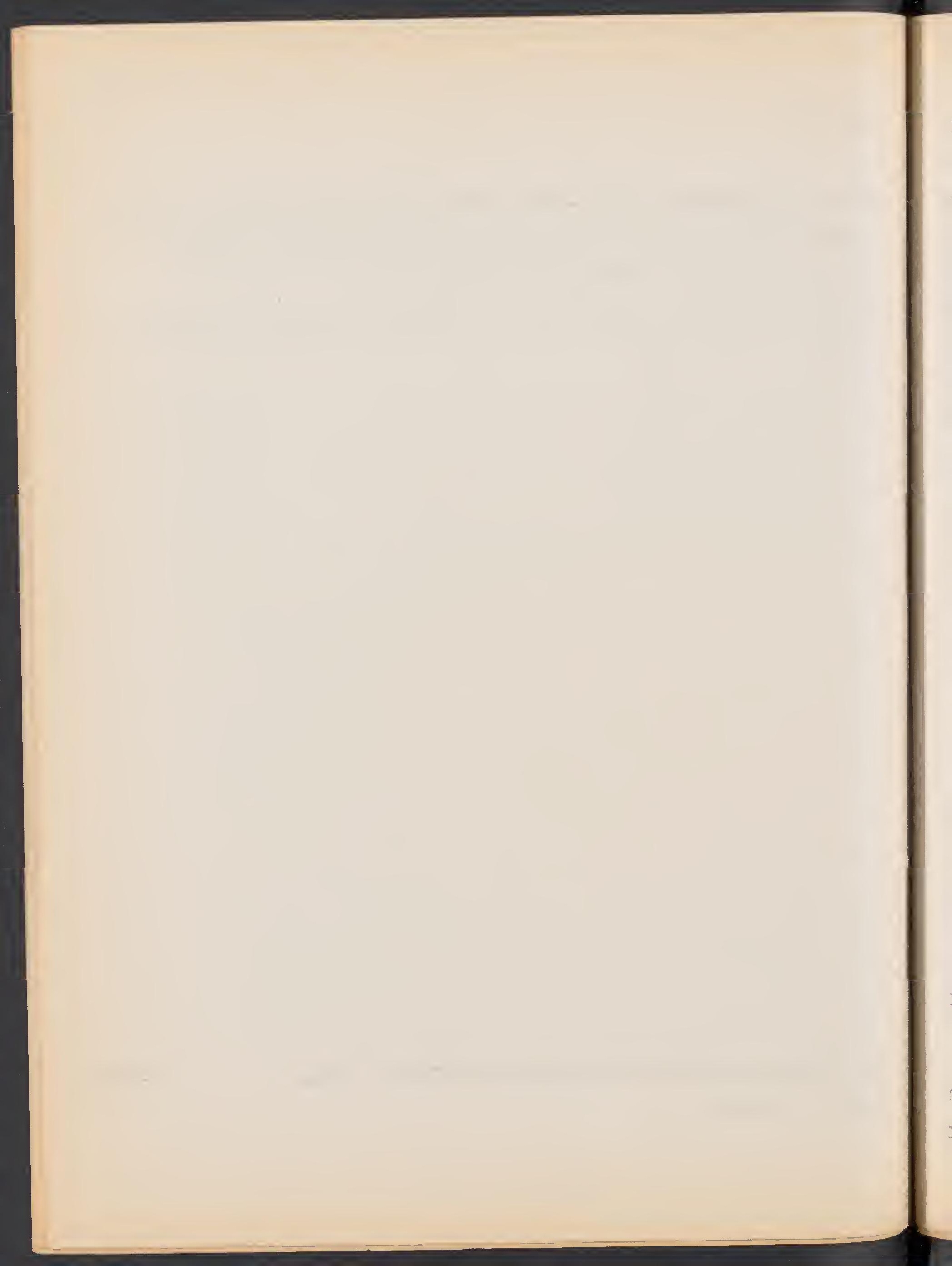


208

In ton $\operatorname{ton}$

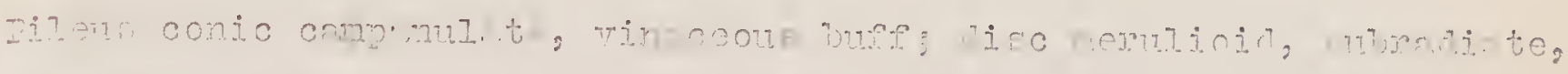

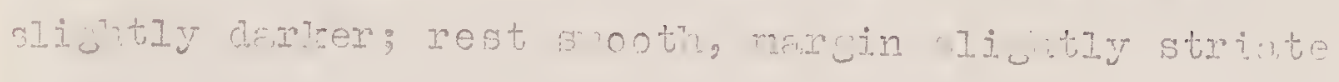
Yilis an te, int, very yoad, croteded stine frafile, wite, $1.7 \mathrm{~cm}$. $=1 \mathrm{~mm}$

Ir corest

Costr alice, ercdi prot, sto Joningo wel rohje: $\varepsilon-7-76$ Dodice froomer 
9.09

Ientoni

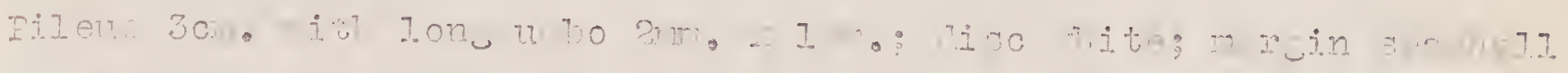

- in?" stricte, Ir,cesate

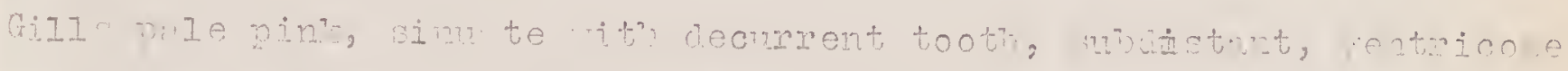

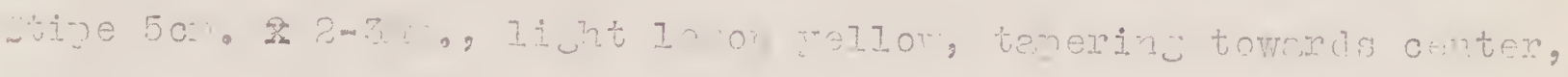

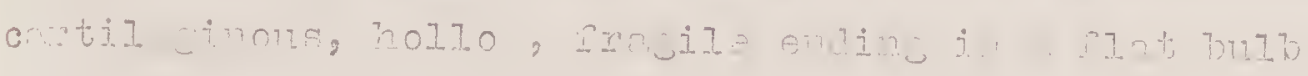

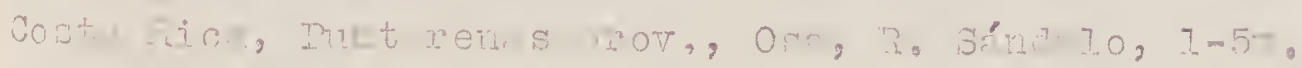
$2-35-$ odge is toercex 


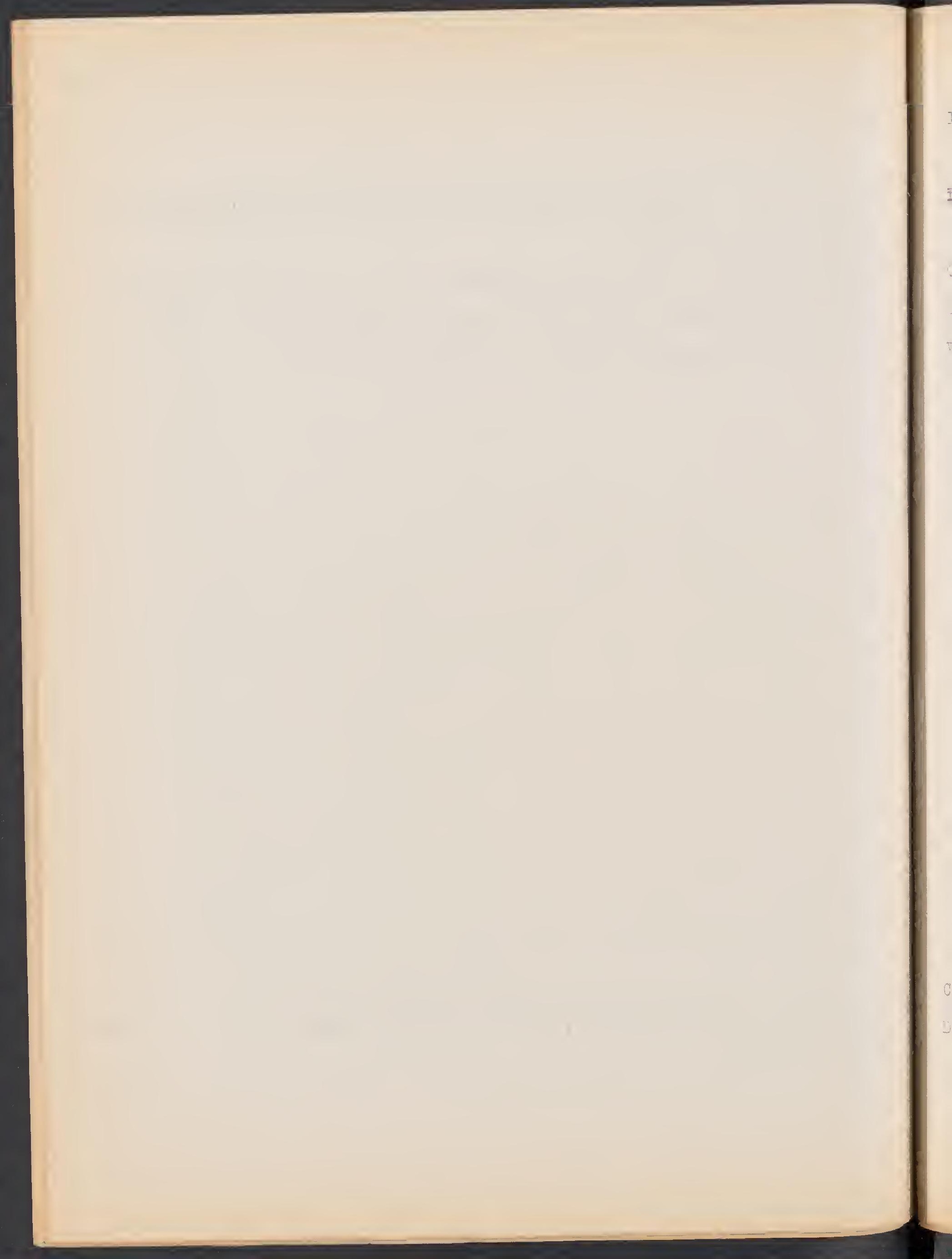


216

T.entoris

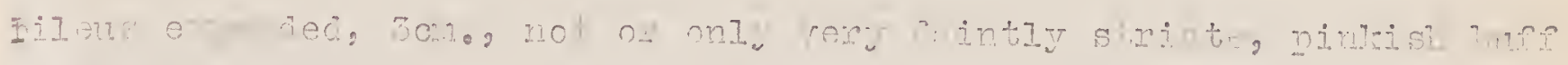

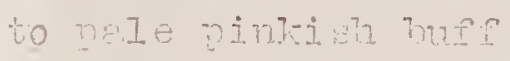

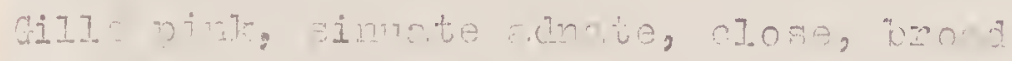

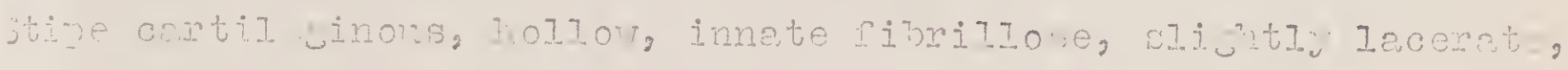

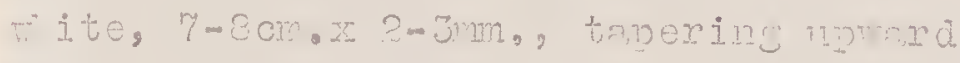

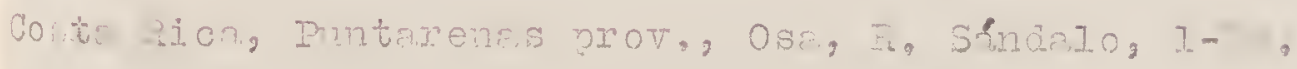

$E-3.7 .79$

Dobs: Goerger 

Iet tonie n. sp.

$21 i$

rileus up to $4 \mathrm{~cm}$ olem, atriate from incipient splitting over sills, translucent seenine somewhat trans arent, disc cloy color, masin pne unligh buef to pintish buff

Gills pele pinkish def to pin ${ }^{\top}$ ist buff, distent, bros rit? maII

nastomosing gilis between, adnete

wtipe pintish buff to top shacing to tawry olive or Jaccardo's umber t buse, up to $4 \mathrm{~cm} \cdot x 3 \mathrm{~mm}$, cartilacinous, hollow, snooth, shining

coste Rica, Iinon orov, Sian Farm, $30 \mathrm{~m}$.

$7-30-36$ 

$21 \%$

W7.

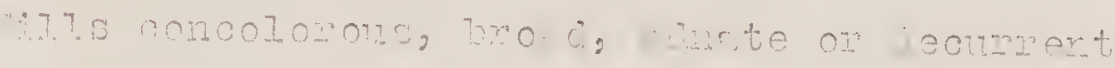

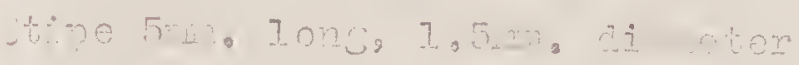

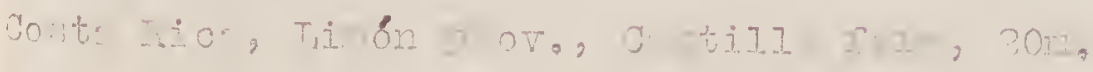

$\eta-j-j$

$20 \cos \operatorname{cosen} 05$ 



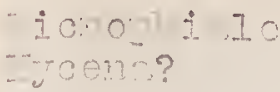

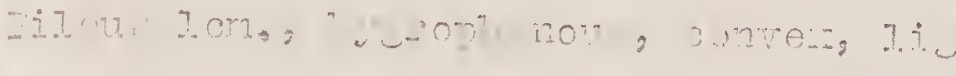
$77+2.8$ ton Fiany y 770 ,

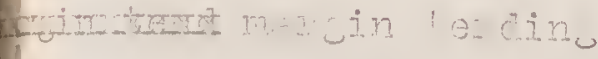

Gills rello oche, n irow, istunt, durte tipe rm, Is nus, mite, cottony, tomentose

Tout wice, Ii ión wot, detizze form, pot. odre w Coercer 

214

Zicroptri. Je

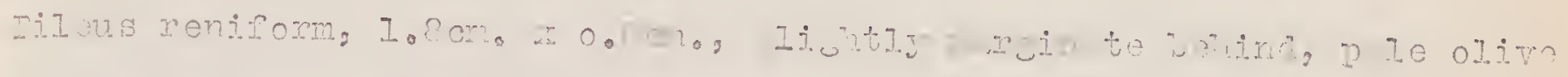
hurf ith lio t yrariath olitre gtatations

Gills inte, not hecurrent

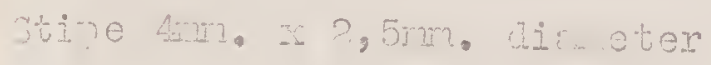

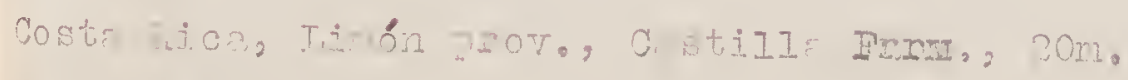

$r 7-2 \varepsilon-36$

blice w roorger 


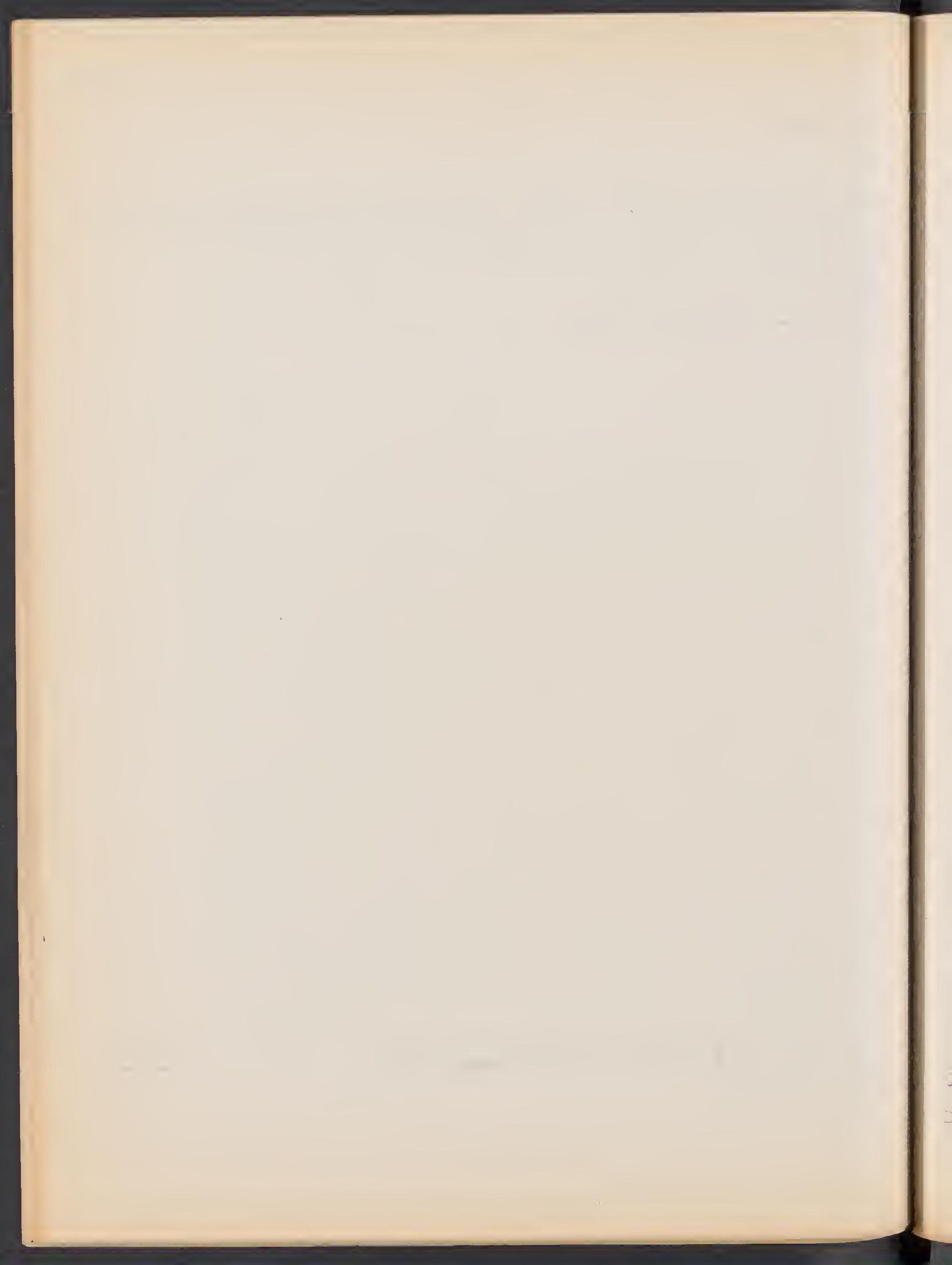


Monotil to

$(0 \cos )=9700.2)$

-11.19. $7-1.70$

$$
\therefore \text { eters, } \therefore
$$

Iis tis to

$\operatorname{t17} 10=9$

IISC Tine ceorle

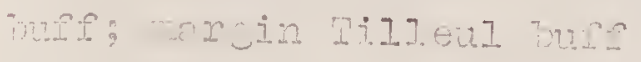

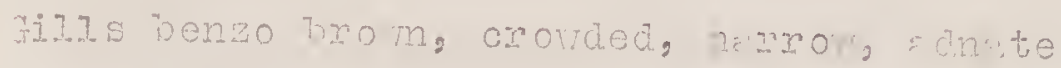

stipe hite, ecceistic, mont ne 5all

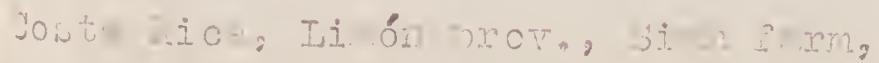

$7-30-36$

Docie woerser 

icropi ie

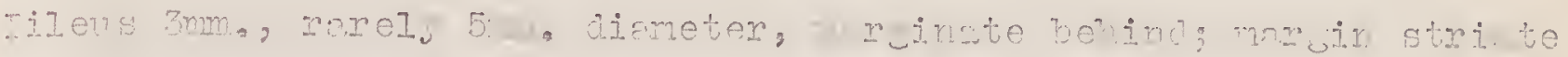

Mils anate, whte, wrom, olove

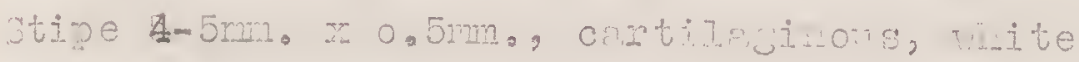

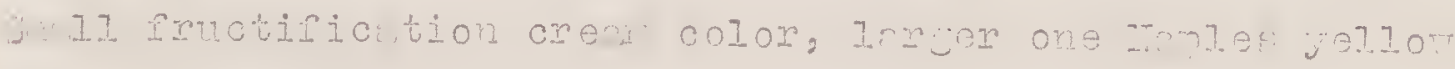

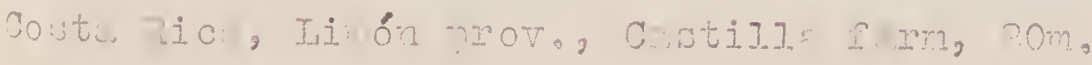

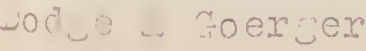




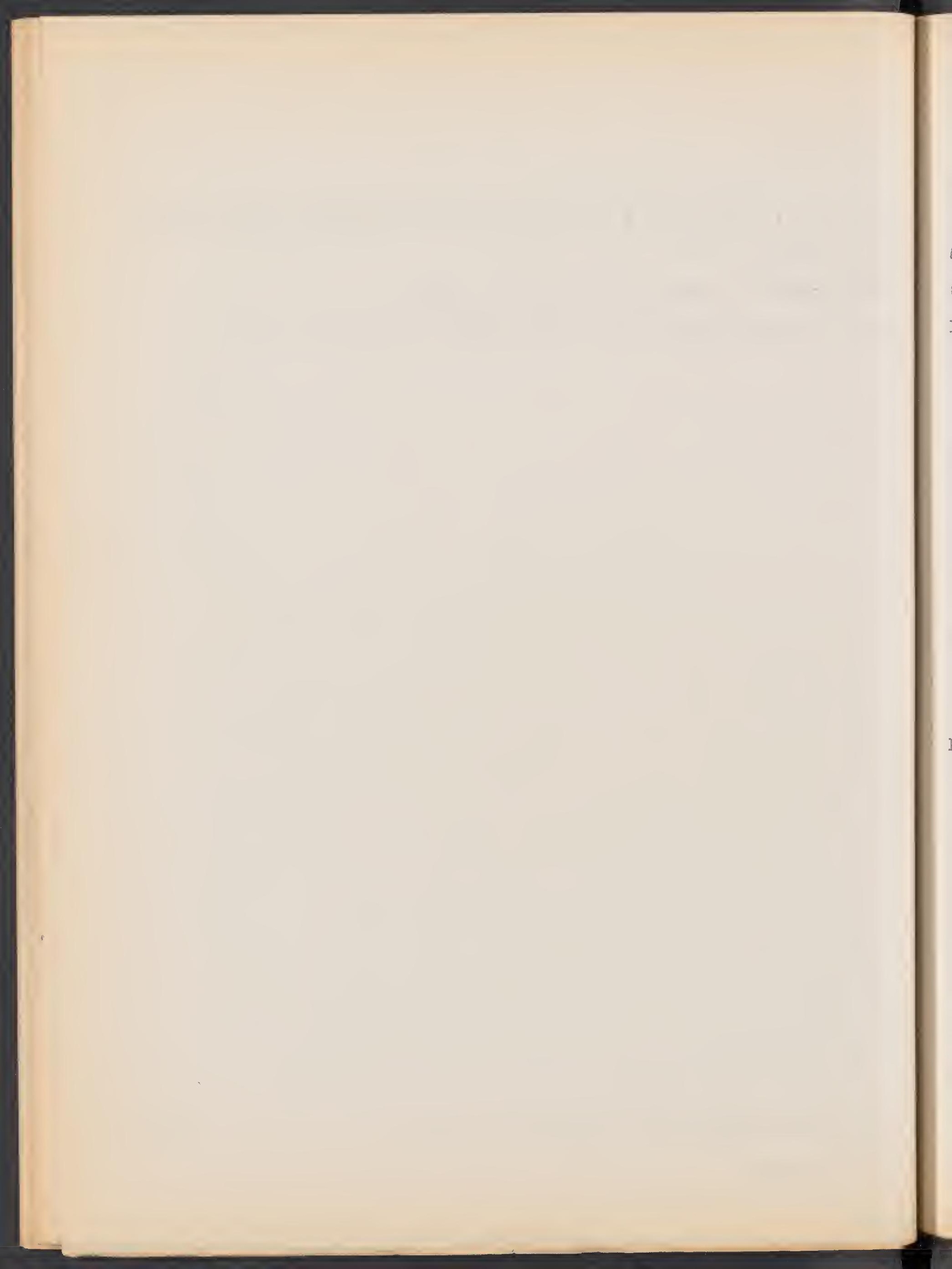


Marasmius

Pileus convex, to flat, $2 \mathrm{~cm}$, minutely velvety, burnt sienna. gills adnate subdistant, narrow, pinkish

stipe cartilaginous tough $4 \mathrm{~cm} \times 1.5 \mathrm{~mm}$, marsbrown to russet and lighter, smooth

Decaying leaves

Puntarenas, Osa, R. Săndalo, $2 \mathrm{~m}$. Dodge \& Goerger 

Marasmius

Pileus up to $2 \mathrm{~cm}$, ochraceous buff to ochraceous tawny with small disc a shade darker than the margin; gills 6-10 in number, light buff to warm buff

stipe cinnamon brown $3 \mathrm{~cm}$ tall less than $1 \mathrm{~mm}$ in diameter

PaIm forest

Limon, Siquirres, Castilla,

Dodge \& Goerger

$7-28$ 


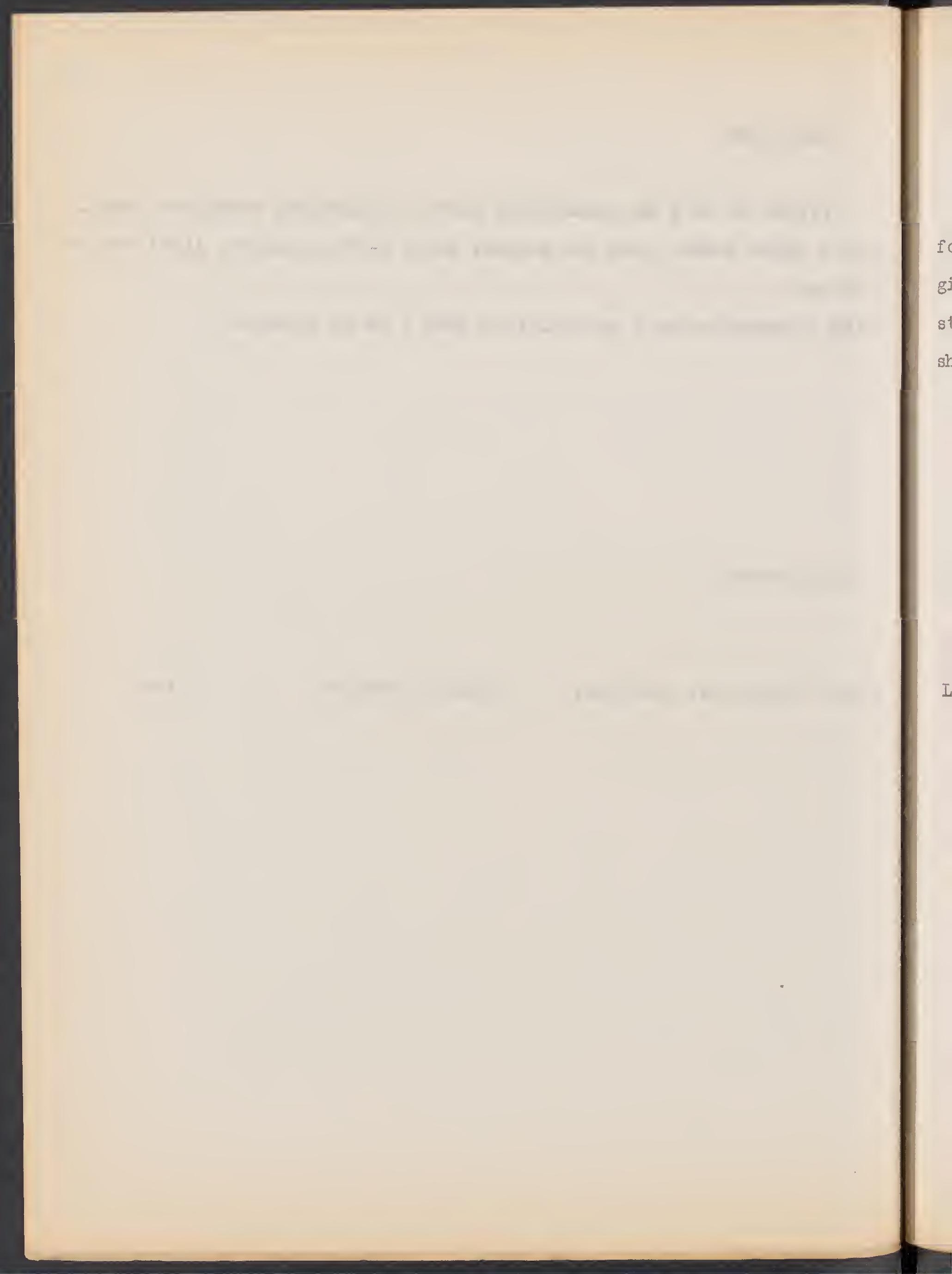


Marasmius

Pileus burnt sienna, $5 \mathrm{~cm}$, expanded central third deeply infundibuli form, velvety pile deeper pile over disc, deeply sulcate over gills gills very broad, wedge shape, eentricose, adnade distant white stipe tough compressed $5 \mathrm{~cm} \times 4-1 \mathrm{~mm}$ tapering downward, white above, $5 \mathrm{~mm}$ shading burnt sienna smooth

Palm forest

Iinon, Siquirres, Castilla 
Marasmius

Pileus hemispheric, deeply sulcate over gills, $1 \mathrm{~cm}$, disc slightly depressed and darker, deep vinaceous; gills pale vinaceous, distant, adnate to collar, narrow; stipe tough, nearly black

Puntarenas, 0sa. R. Sándalo, $2 \mathrm{~m}$. Dodge \& Goerger $8-23$ 


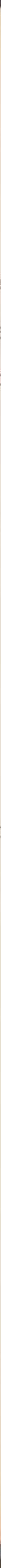


inarasmius

Pileus convex, $6 \mathrm{~mm}$, strigose fibrillose, not striate, slightly umbilicate, Saccardo's umber shading cinnamon buff; gills white, ventricose close, sinuate adnate stipe concolor, minutely strigose, $4 \mathrm{~cm}$ long

R sandalo

Funtarenas, 0sa, 2 m., Dodge \& Goerger 


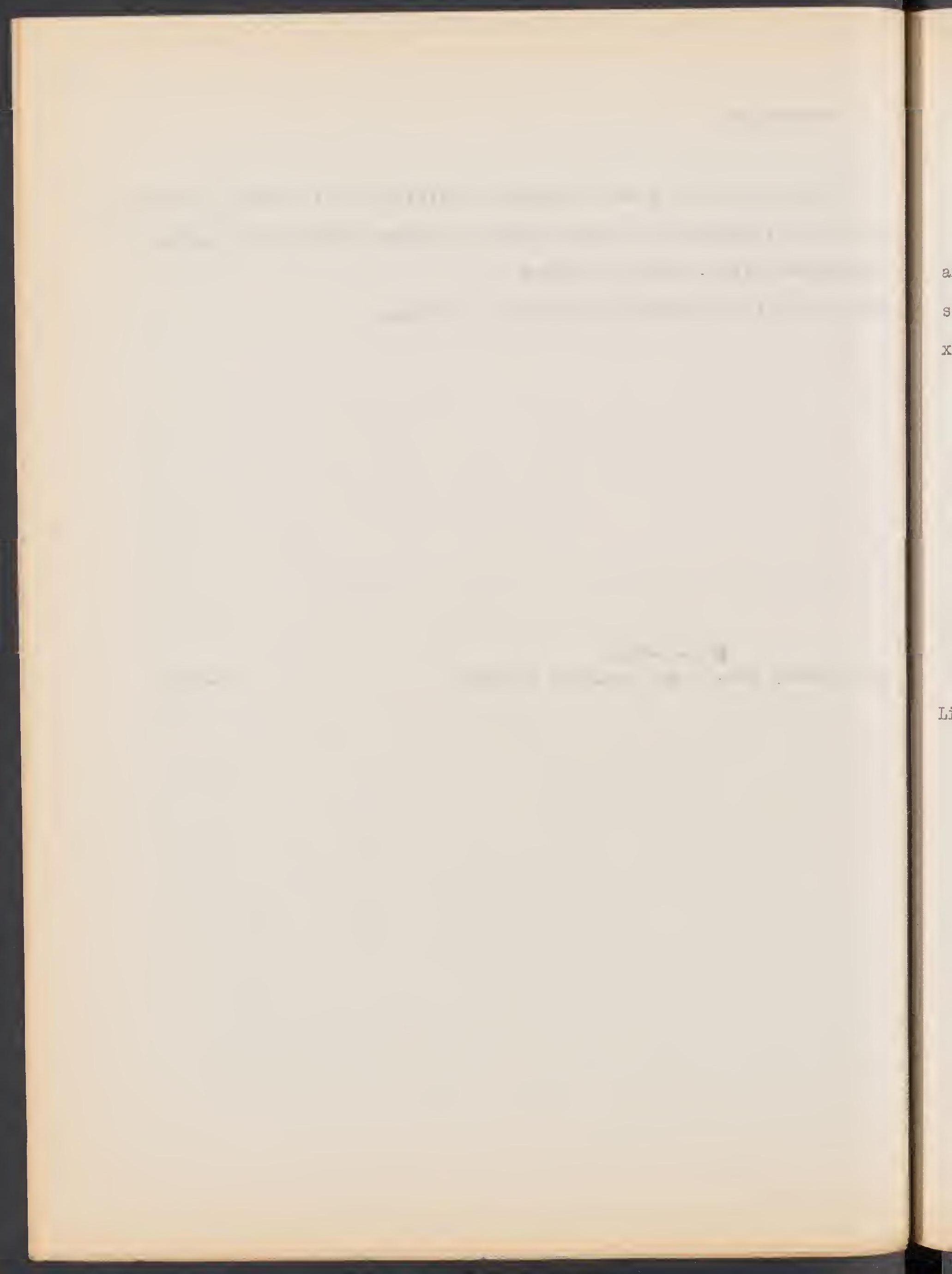


Marasmius

Pileus about $18 \mathrm{~mm}$, stiate sulcate, disc yellow ochre, margin antimony yellow; gills tarm buff, distant, adnate to collar, broad; stipe warm buff above shading to ochraceous tawny and cinnamon brown, $4 \mathrm{~cm}$ $x 0.5 \mathrm{~mm}$, shining, base cottony

Limon, Siquirres, Castilla

Dodge \& Goerger

$7-23$ 


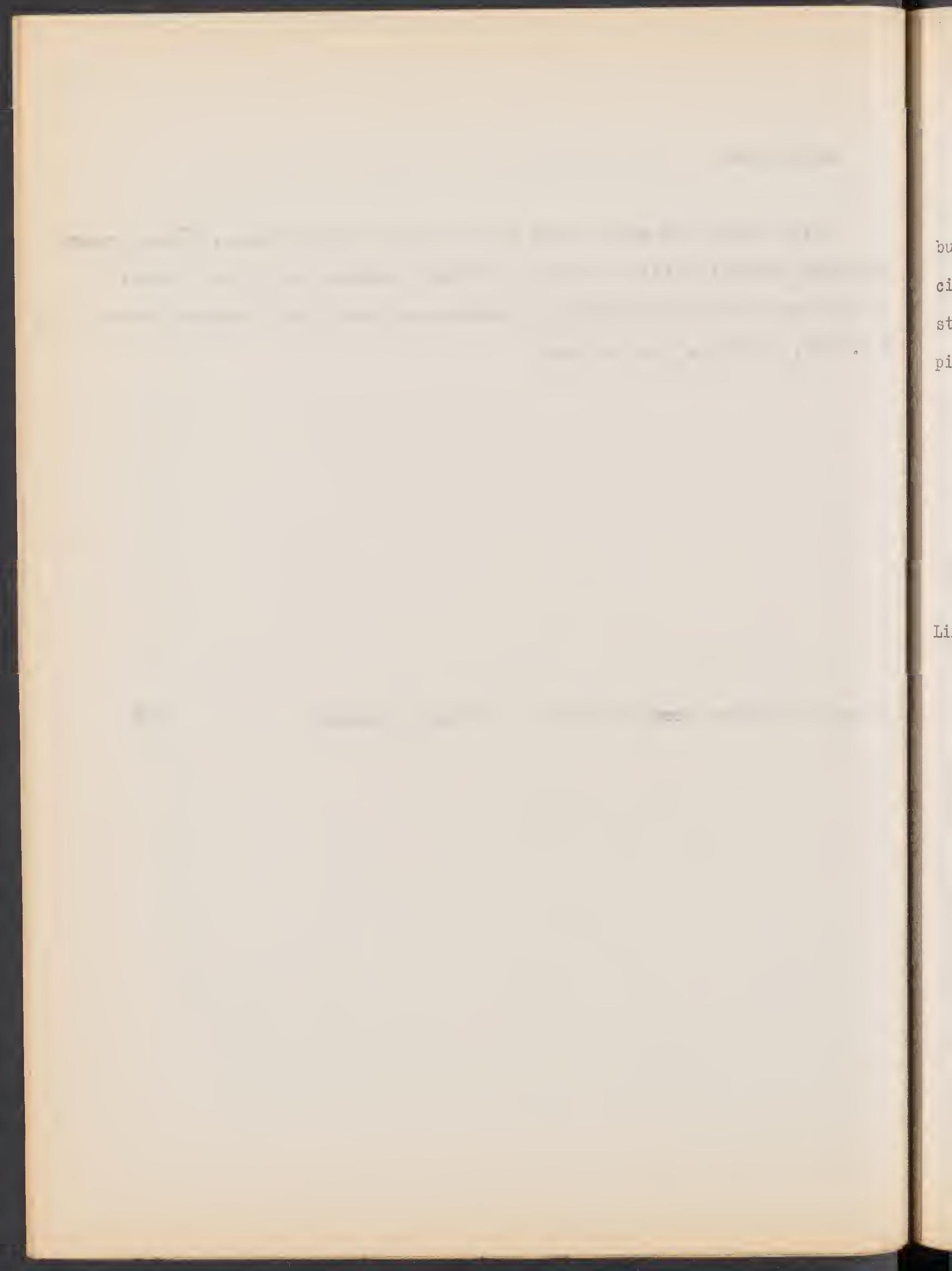


Marasmius

Pileus $1.5-2 \mathrm{~cm}$, convex, center depressed becoring broadly infundibuliform, hygrophanous, disc pale pinkish cinnamon, margin striate, cinnamon; gills distant, pale pinkish cinnamon thick stipe $2.5 \mathrm{~cm} \times 3-1 \mathrm{~mm}$. tapering wownward, somewhat compressed, pale pinkish cinnamon above, burnt umber below

Limon, Siquirres, Castilla, July 23, Dodge \& Goerger 


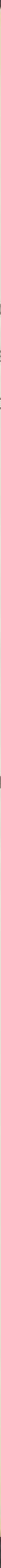


Marasmius

Pileus expanded, $2 \mathrm{~cm}$ in diameter, disc merulioid, clay color, rest originally velvety sepia soon splitting down backs of gills showing alternate triangles of pinkish buff; gills very distant, pinkish buff, adnate to collar; stipe $4 \mathrm{~cm}$

Puntarenas, 0sa, R. Sándalo, 2 m. Dodge \& Goerger $8-24-36$ 


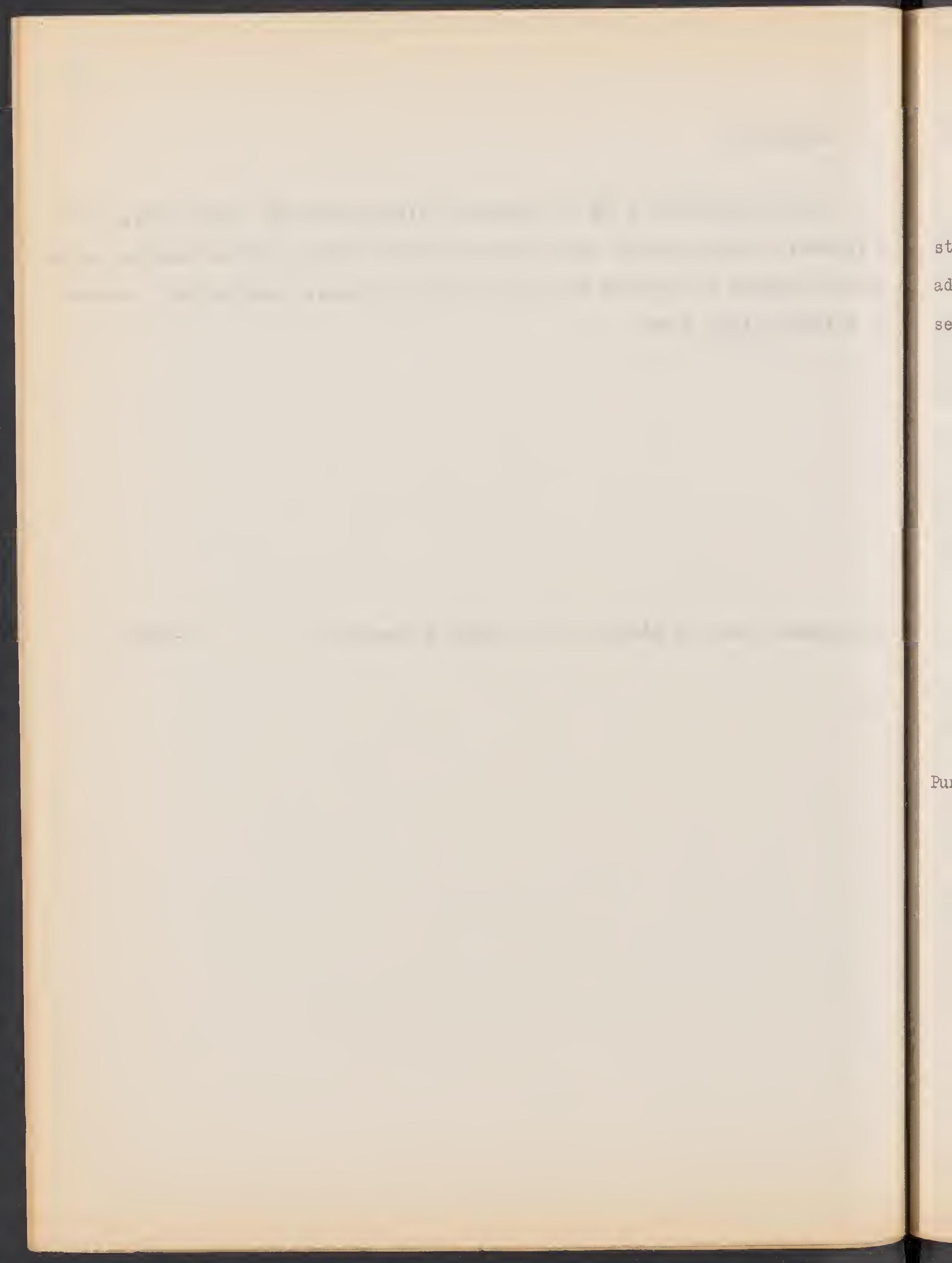


Marasmius

pileus convex to flat, disc depressed, small, white or nearly so, stri striate sulcate over gills, Verona brown, warm sepia over gills which are adnate to collar, subdistant, narrow, white; stipe cartilaginous tough, semipellucid, 4 cm $\times 2.5-1.5 \mathrm{~mm}$ tapering upward, light vinaceous cinnamon

Decaying leaves

Puntarenas, 0sa, R. Sándalo, $2 \mathrm{~m}$. Dodge \& Goerger 


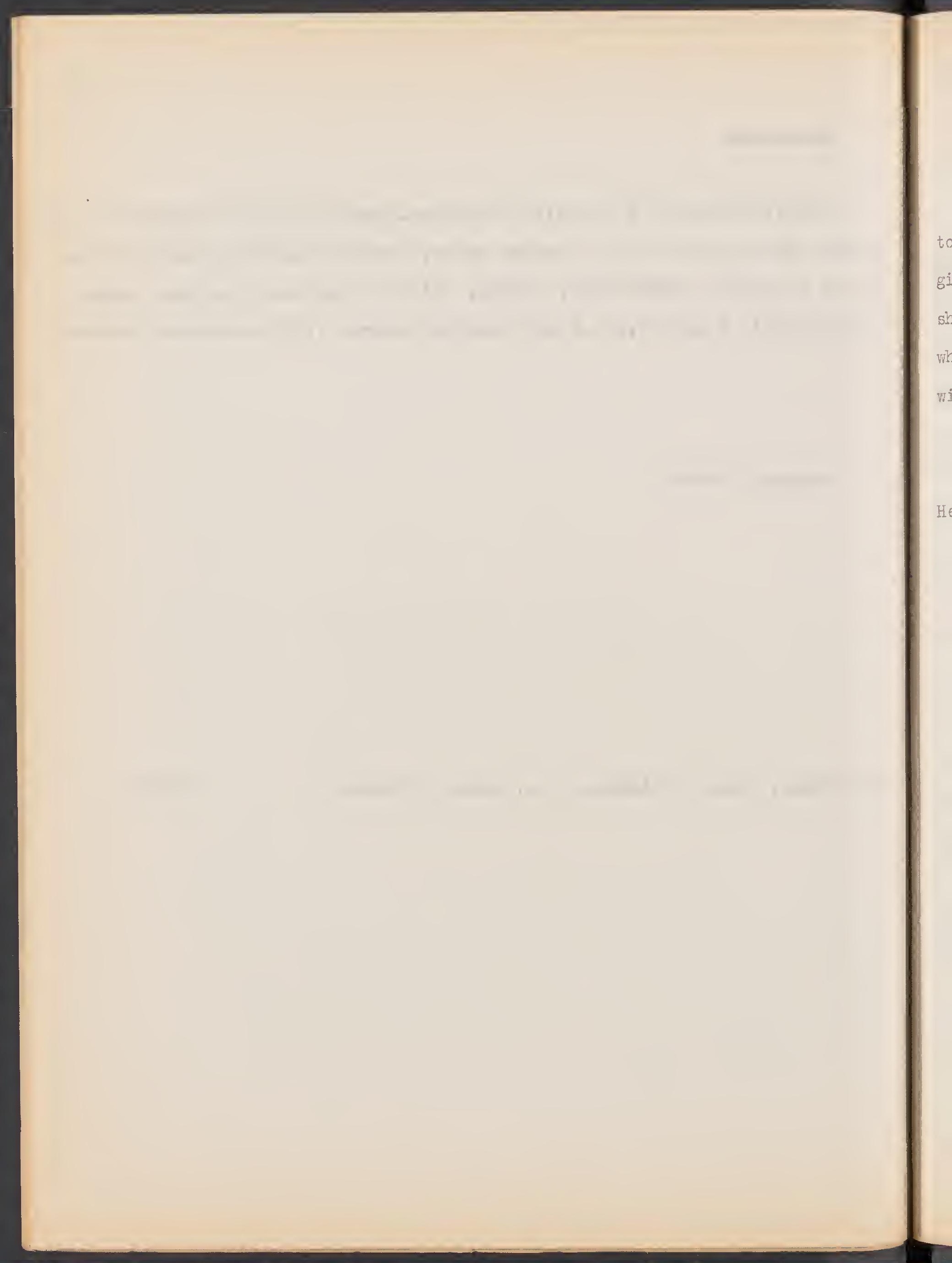


Marasmius

Pileus $1.5 \mathrm{~cm}$ in diameter, hemispheric, disc flesh ochre shading to cartridge buff margins, substrigose, velvety, margin striate sulcate; gills white, close adnate broad; stipe 2.3 cr $x 1 \mathrm{~mm}$, white at apex shading to seal browmase, fibrillose at apex ending in small brow dots where pileusbreaks away; pseudo ring at attachment of gills, rest velvety with scattered strigose white hairs

Heredia, Chahuites, $1600 \mathrm{~m}$., Goerger 

Marasmius

Pileus smoke gray, disc much darker, margins elevated and more or less infundibuliform, rugose sulcate over gills which are white, distant, broad, more or less anastomosing. stem black fuscous $2 \mathrm{~cm} x \mathrm{~mm}$ 


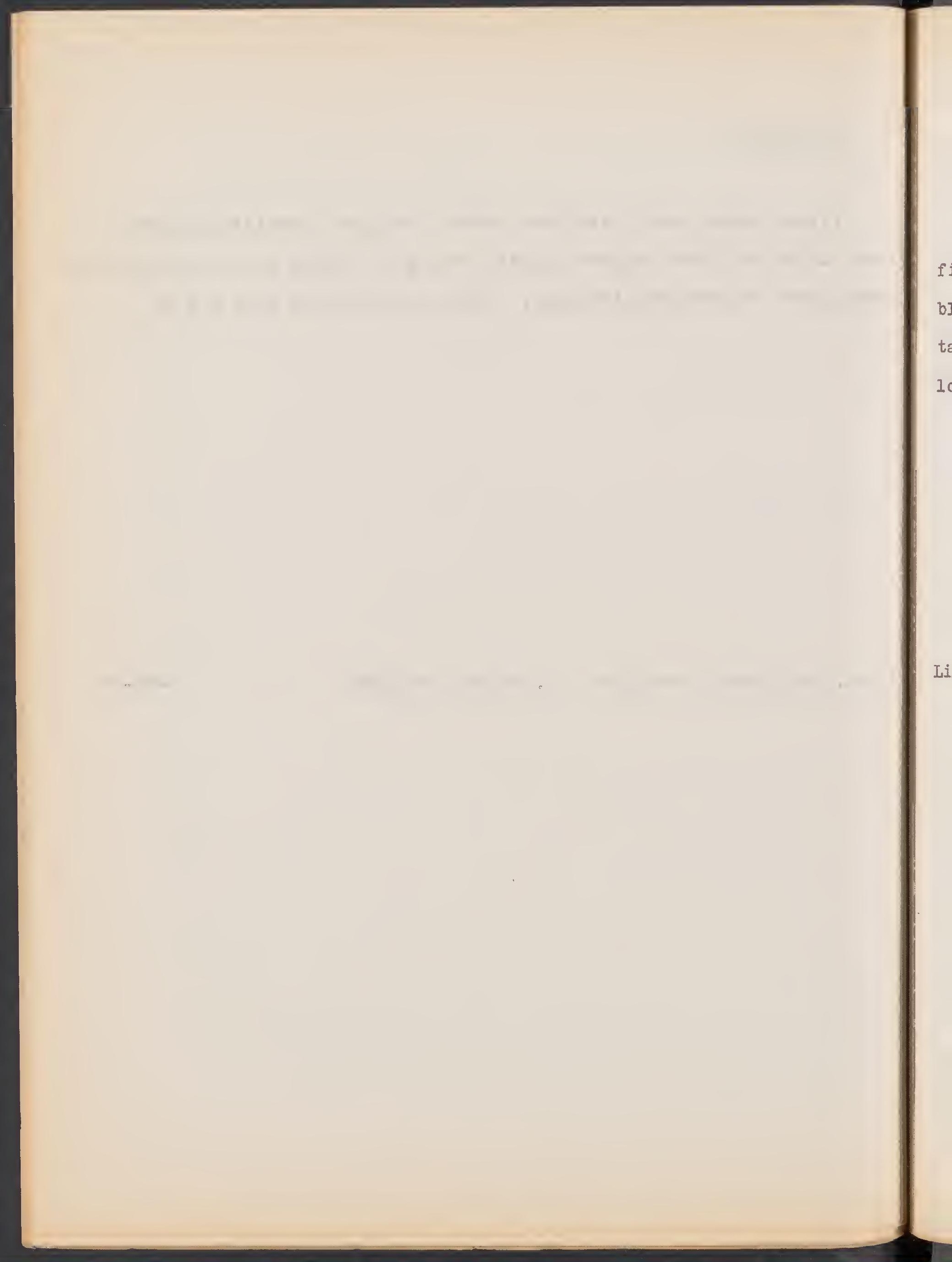


Mycena

Pileus $1.2 \mathrm{~cm}$, convex, center depressed with small sharp conic umbo, fibrillose tomentulose, margins striate pale windsor blue with nigrosin blue umbo; gills adnate, broad, subdistant, white; stipe $2.5 \mathrm{~cm} \times 1 \mathrm{~mm}$ tapering slightly downward, cartilaginous tough, light windsor blue above lower portion aniline yellow

Limon, Castilla, Dodge \& Goerger $7-25$ 


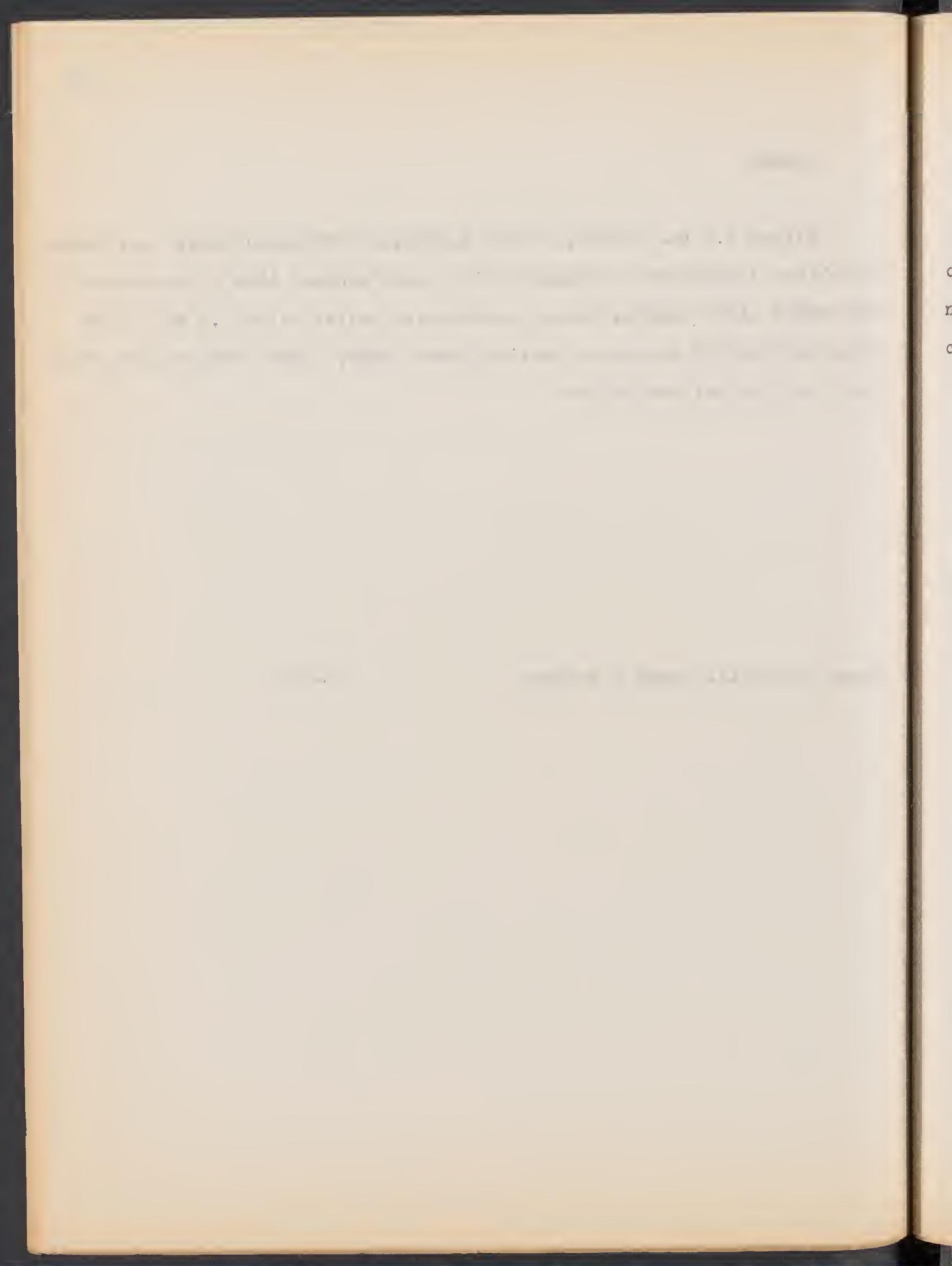


Mycene.

Pileus $1 \mathrm{~cm}$, hygrophanous, wholly striate over gills, disc pinkish cinnamon, striae snuff brown, pale pinkish cinnamon between; gills adnexed, broad, close, white; stipe white, fragile pruinosex above, silky cartilaginous below, $1.5 \mathrm{~cm} \times 1 . \mathrm{mm}$.

On decaying polypore

Puntarenas, Osa, R. Sándalo, Dodge \& Goerger $8-22-36$ 


\section{Mycena.}

Pileus hemispheric, $8 \mathrm{~mm}$, very minutely velvety, bay; gills broad, white, adnate, distant; stipe $1.5 \mathrm{~cm} \times 1.5 \mathrm{~mm}$, cartilaginous, lighter above, bay below

Puntarenas, Osa, R. Sándalo, Dodge \& Goerger 
Mycena

'Pinérs $1.2 \mathrm{~cm}$ in diameter, convex, striate, sulcate, magins disc flat bewn beown, rest buckthorn brown; gills white, adnate, thick subdistant; stipe wite above, I cm $\times 1 \mathrm{~mm}$, mummy brown below, cartilaginous

Decaying leaf

Puntarenas, Osa, R. Sándalo, Dodge \& Goerger 8-24-36 

Mycena

Pileus up to $1.2 \mathrm{~cm}$ in diameter, expanded, hygrophanous, smooth, margin narrowly striate, buffy brown, margin clowe brown; gills broad, adnate, semipellucid, close; stipe $1 \mathrm{~cm} \times 1 \mathrm{~mm}$, cartilaginous, fragile, silky white

Puntarenas, Osa, R. SándaIo, Dodge \& Goerger 


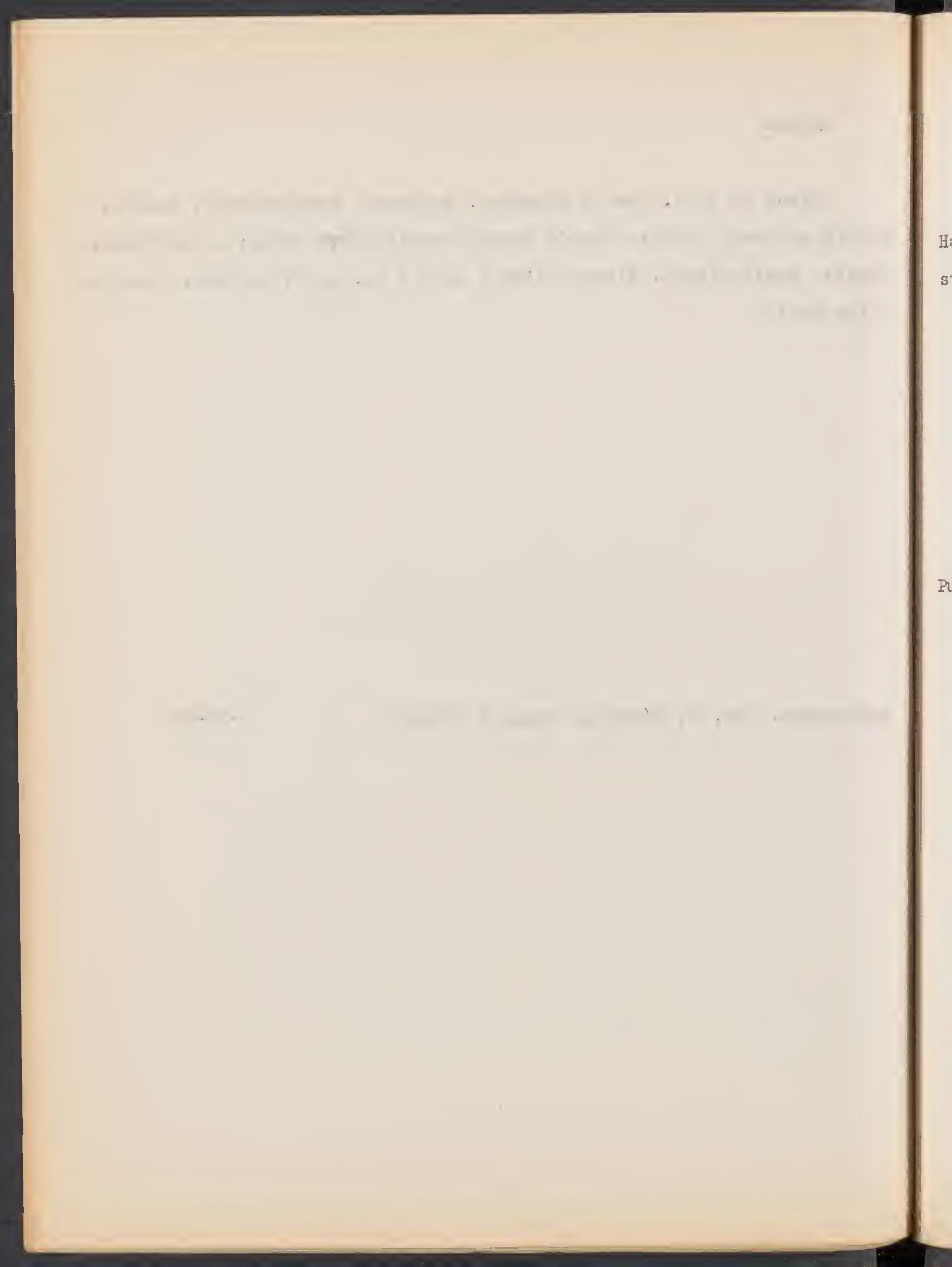


Mycena

Pileus $5 \mathrm{~mm}$. hemispheric, subcampanulate, margin striate sulcate, Hays' russet; gills adnate to subdecurrent, close, white, narrow; stipe $1.3 \mathrm{~cm} \times 0.5 \mathrm{~mm}$, cartilaginous, flesh color

Puntarenas, Osa, R. Sandalo, Dodge \& Goerger 

inycena

Pileus campanulate, stiriate, Very thin, $1.2 \mathrm{~cm}$ diam, $7 \mathrm{rm}$ tall, umbilicate deeply, light brownish olive; gills broad, white distant, adnate; stipe white, cartilaginous $3.5 \mathrm{~cm} x I \mathrm{~mm}$. fragile 

Mycena

Pileus flat, margin striate sulcate, disc dark purple drab to light purple drab margins; gills broad, adnate, distant, pale pinkish drab stipe white cartilaginous tough, $1.5 \mathrm{~cm} \times 1 \mathrm{~mm}$ taperingdownward 


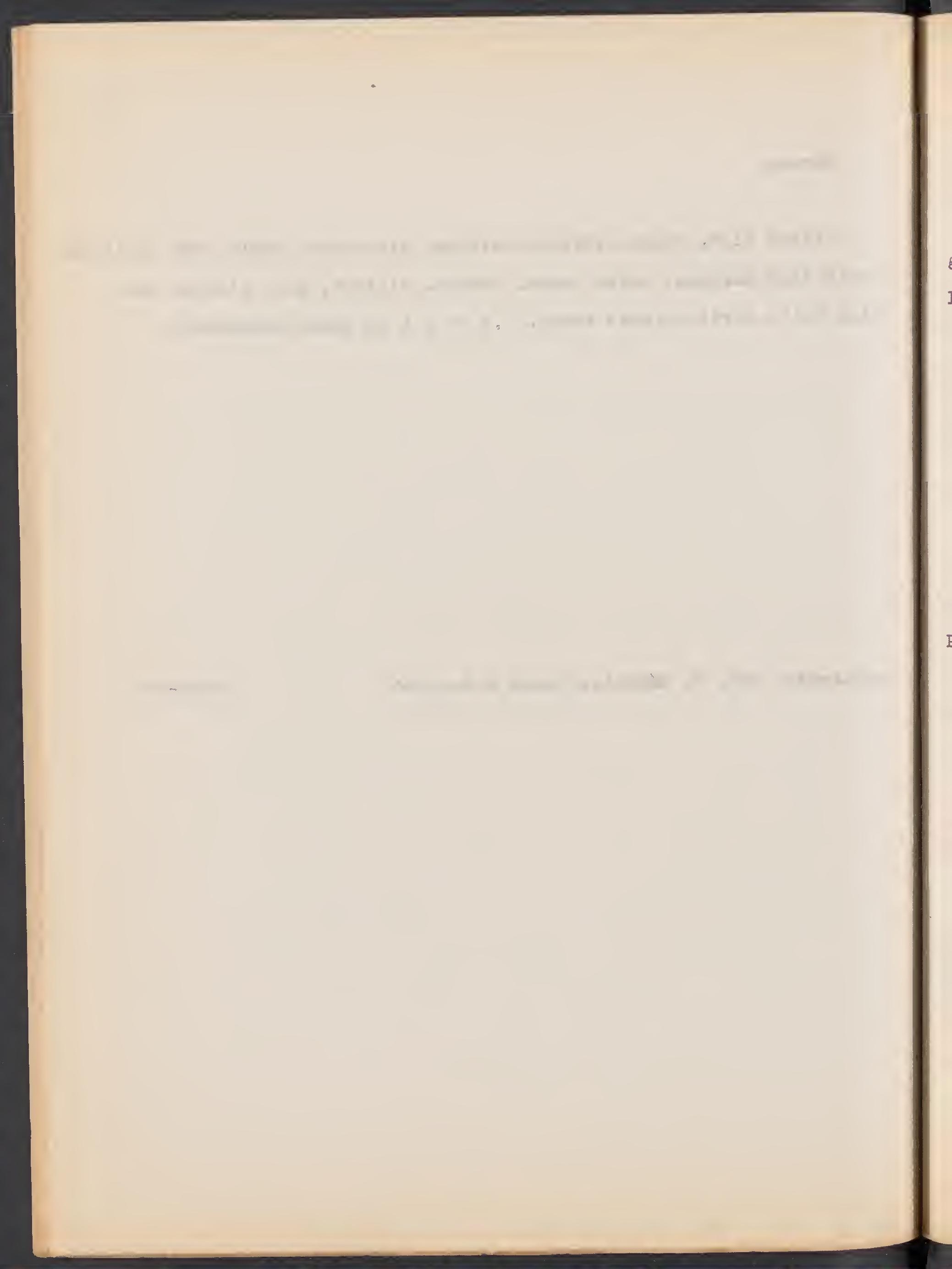


Nycena

Pileus flat, 8-9 mm, striate, innate fibrillose, Saccardo's umber gills subdistant, white, broad, sinuate adnate, hygrophanous; stipe $1.5 \mathrm{~cm} \times 0.5 \mathrm{~mm}$, white cartilaginous 


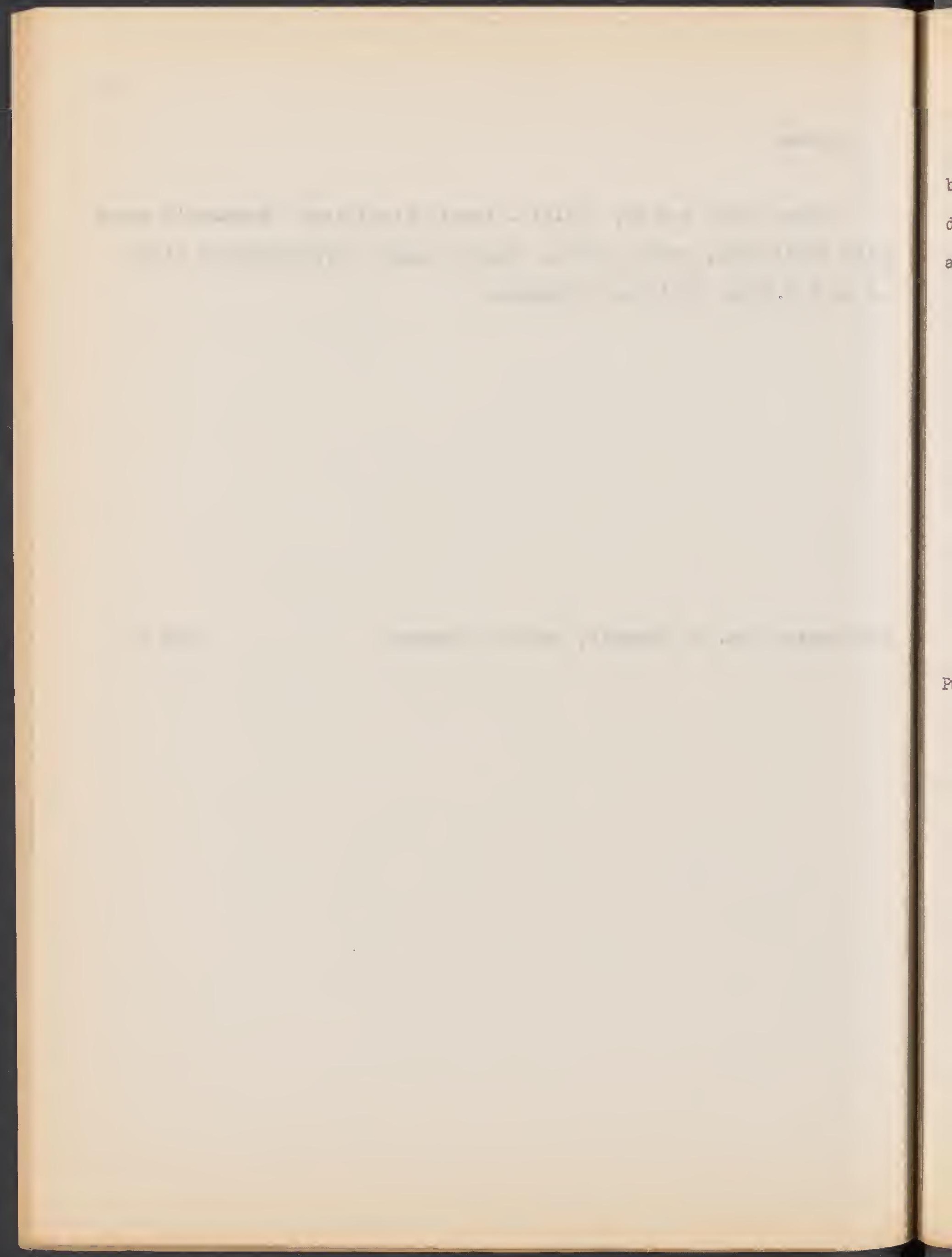


Mycena

Pileus convex to flat, margins upturned, striate to disc, mars brown to light ochraceous salmon, gills adnexed, broad, hygrophanous, subdi distant, ochraceous salmon, stipe cartilaginous $2 \mathrm{~cm} \times 2 m m$, concolor above, mars brown or darker below

Puntarenas, Osa, R. Sandalo, Dodge \& Goerger 


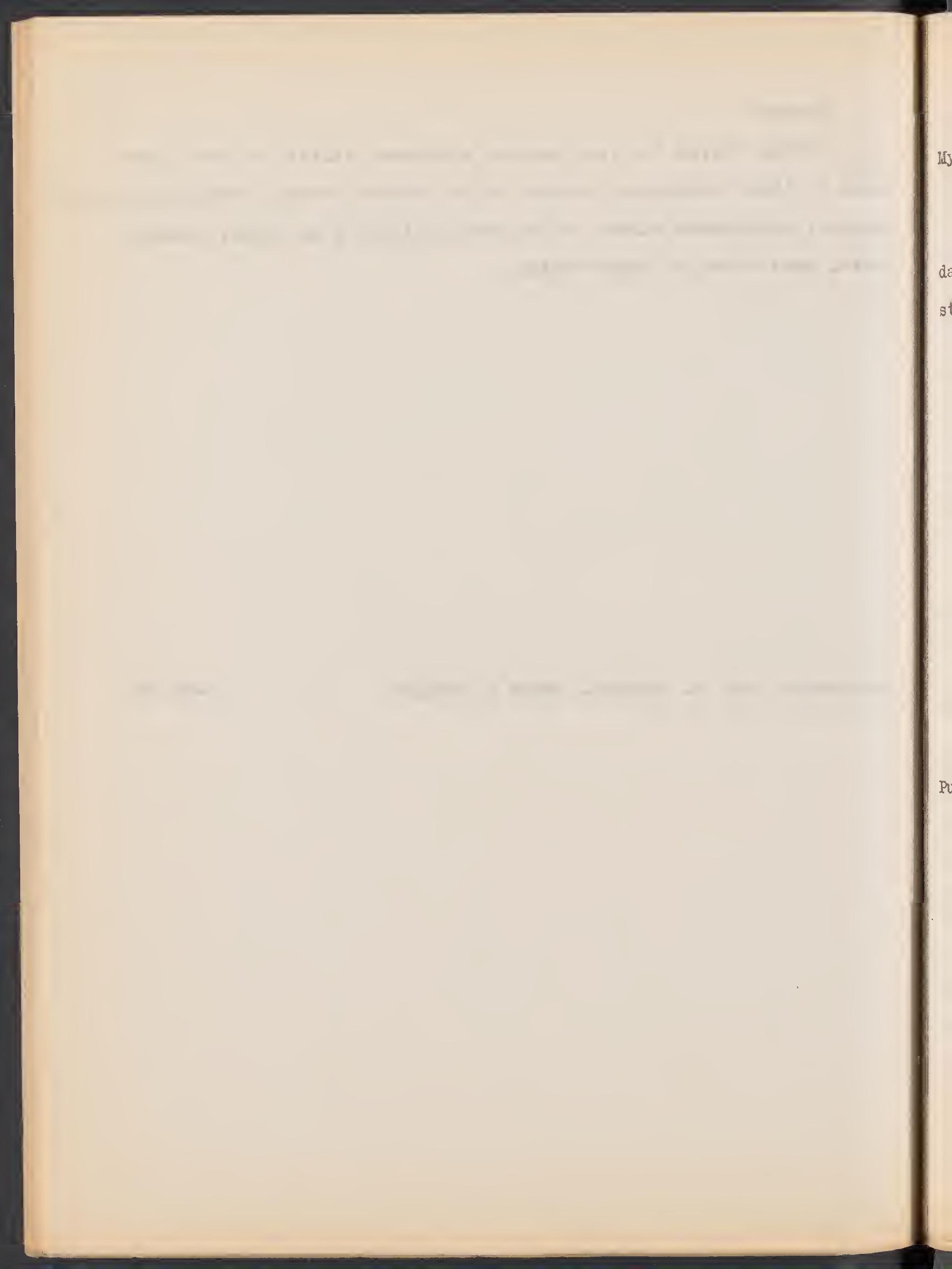


Mycena

Pileus $7 \mathrm{~mm}$, hemispheric, deeply umbilicate, brownish olive or darker, silky fibrillose; gills subdistant, adnate, broad, white; stipe silky fibrillose, concolor, $4 \mathrm{~cm} \times 1.5 \mathrm{~mm}$. 


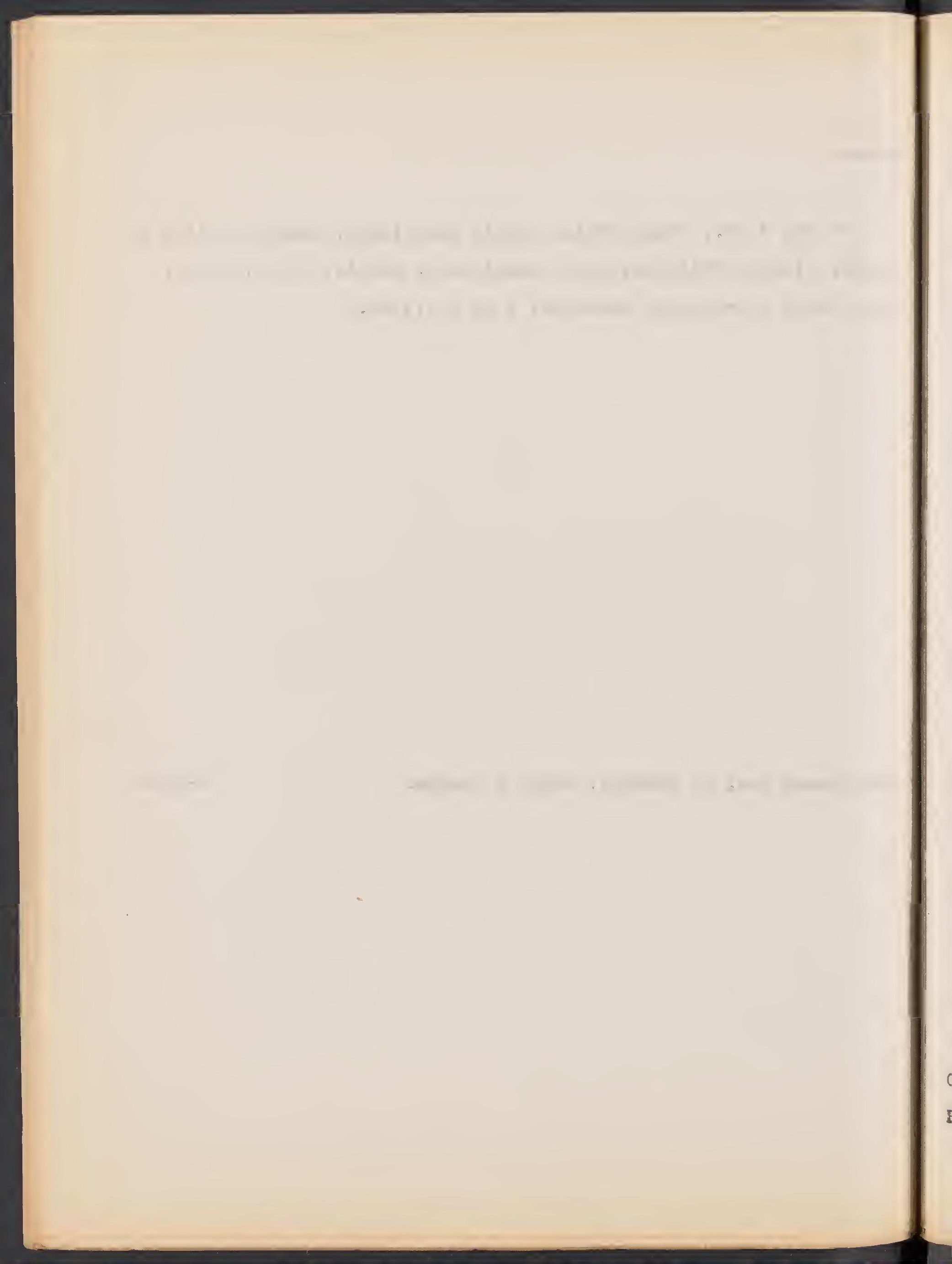


Mycena

Dictyolus

Pileus expanded, nearly flat, 7mm., spongy strigose; margin outrolled; between drab gray and smoke gray

Gills white, distant broad ridges only slightly elevated, adnate Stipe cartilaginous, white, fragile, $7 \mathrm{rm}$, tall $\times 1 \mathrm{~mm}$. in diameter

Costa Rica, Puntarenas prov., 0sa, R. Sándalo, 1-5m. 


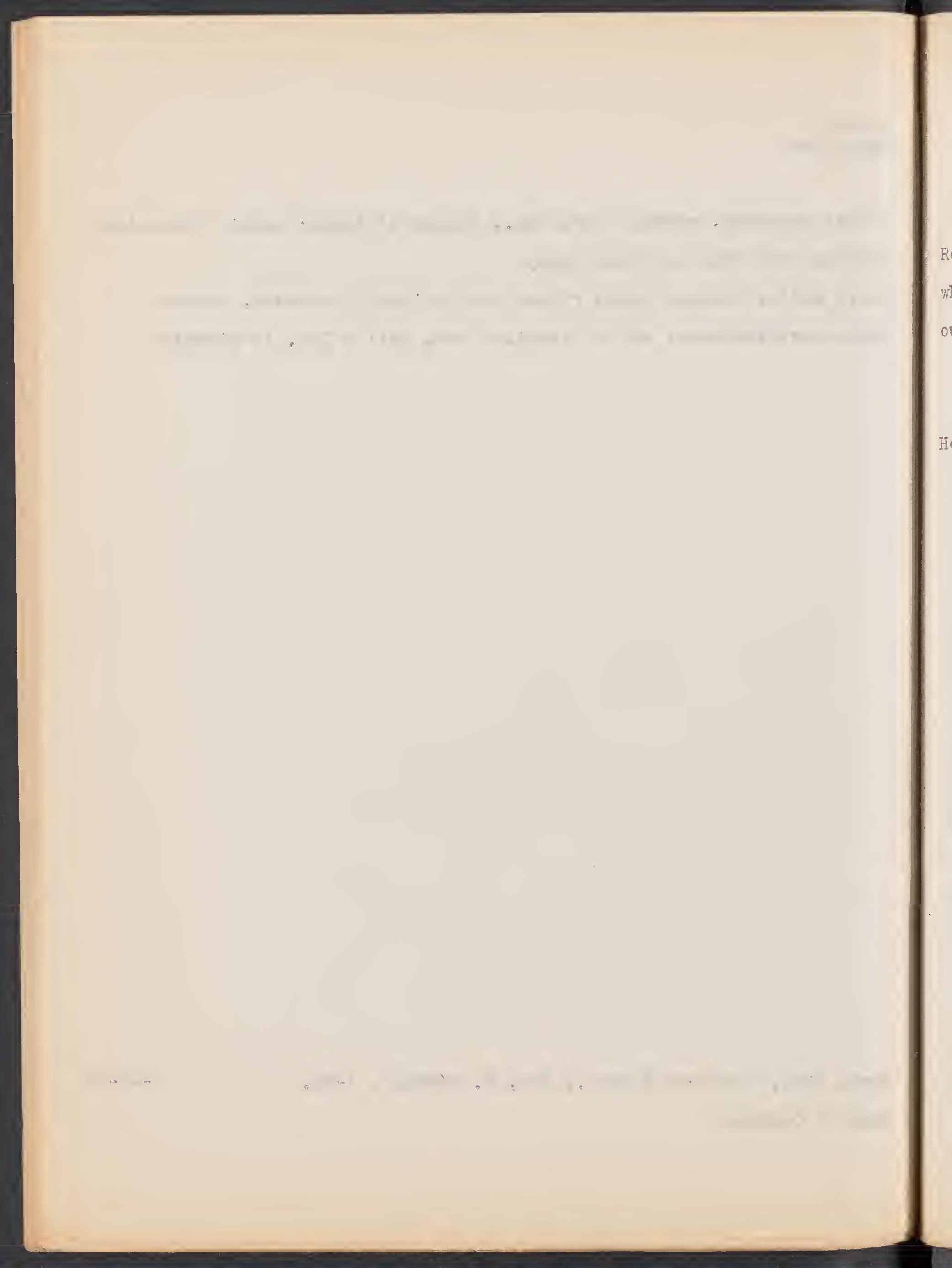


Inycena

Pileus convex, $1.5 \mathrm{~cm}$, minutely idexzinnate sealy, buff pinktto Roods brow, margin striate and lacerate; gills ventricose, edges eroded white, close adnate; stipe buff pink or darker, $4 \mathrm{~cm} \times 2 \mathrm{~mm}$, cartilaginous, holIow

Heredia, Chahuites, $1600 \mathrm{~m}$., Goerger $9-11-36$ 

Mycena

Pileus expanded to nearly flat, deeply splitting along backs of gills, surface pruinose, drab gray, $3 \mathrm{~cm}$. in diameter

Gills subdistant, pale drab gray, broad, adnate

Stipe very slender and fragile, $5 \mathrm{~cm}$. or more in length, white

Among decaying trash on soil in bananal

Costa Rica, Puntarenas prot., R. Paquita

Dodge \&s Goerger 


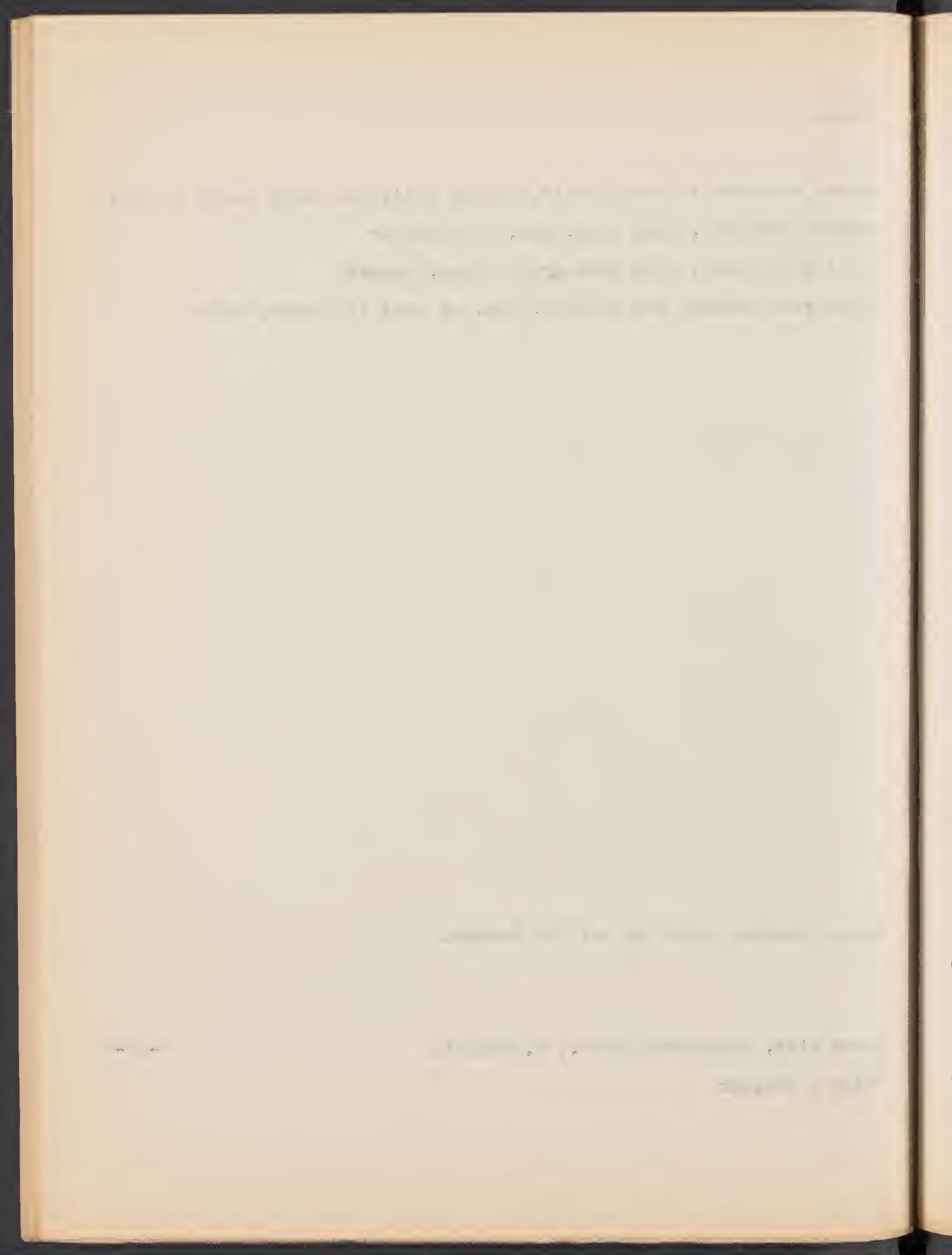


Mycena

Pileus hemispheric to convex, hygrophanous, up to 6mm. in diameter, pale pinkish buff

Gills adnate, narrow with still. narrower cross gills, subdistant, white Stipe 2cm. x Imm., semipellucid, white, cartilaginous, fragile

Gregarious on decaying Iog

Costa Rica, Puntarenas prov., Osa, R. Sándalo, 1-5m. 

245

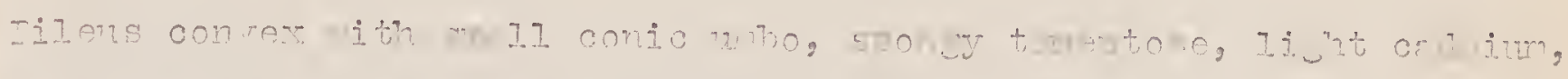

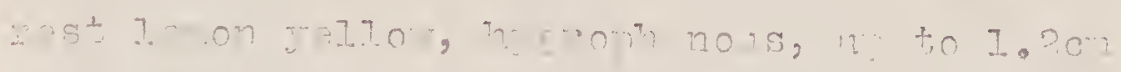

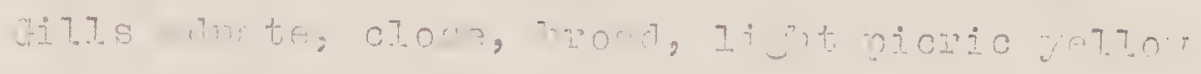

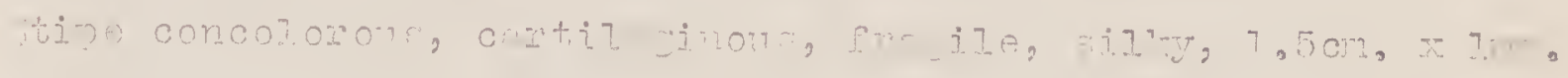

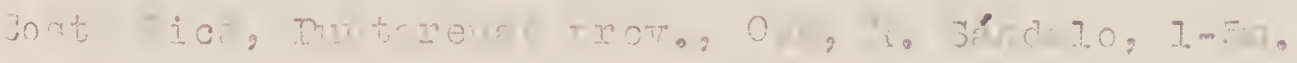

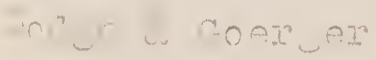



Proena

244

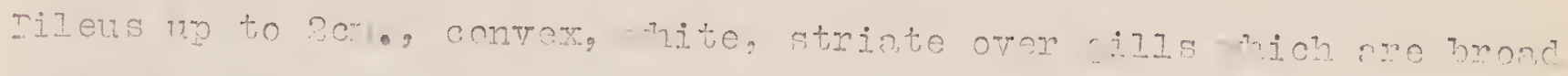

inotant, white steivinc radish whon old

otipe alonder, Icm. toll, morrocoo red, touth

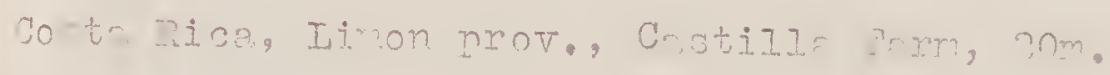

$7-25-39$ 



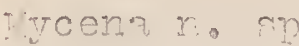
nesm Trojonn

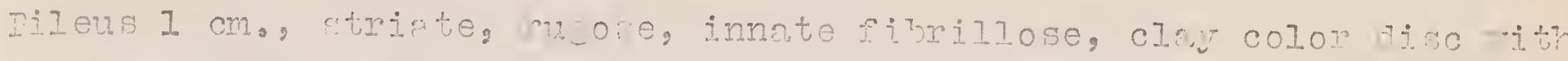
cimnaron buff ingrin

Hil a bropd, sibeictent, einunte, nonste, whjte

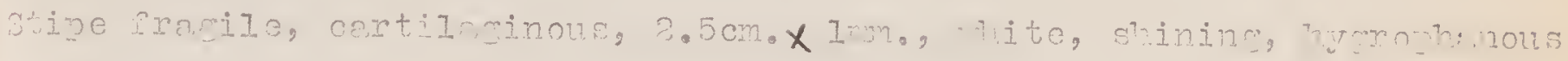

In poIm rorest 

Tro

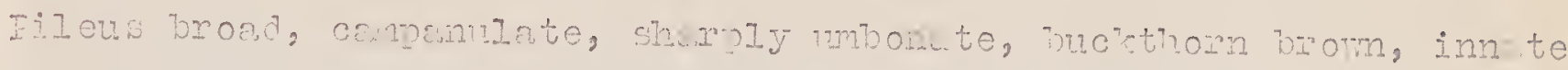
fibriliose, rugose, striete; nomins ginute, I coerte

Gills brocd, subdistant, c, dinate, wite

Stipe cartilacinous, wite, $6 \mathrm{~cm} . X_{0} 0.5 \mathrm{~min}$, ailhy

Worest along tram Iine

vosta Rica, Linon vrov. Sian Farn, ?0 m. $7-30-36$ 


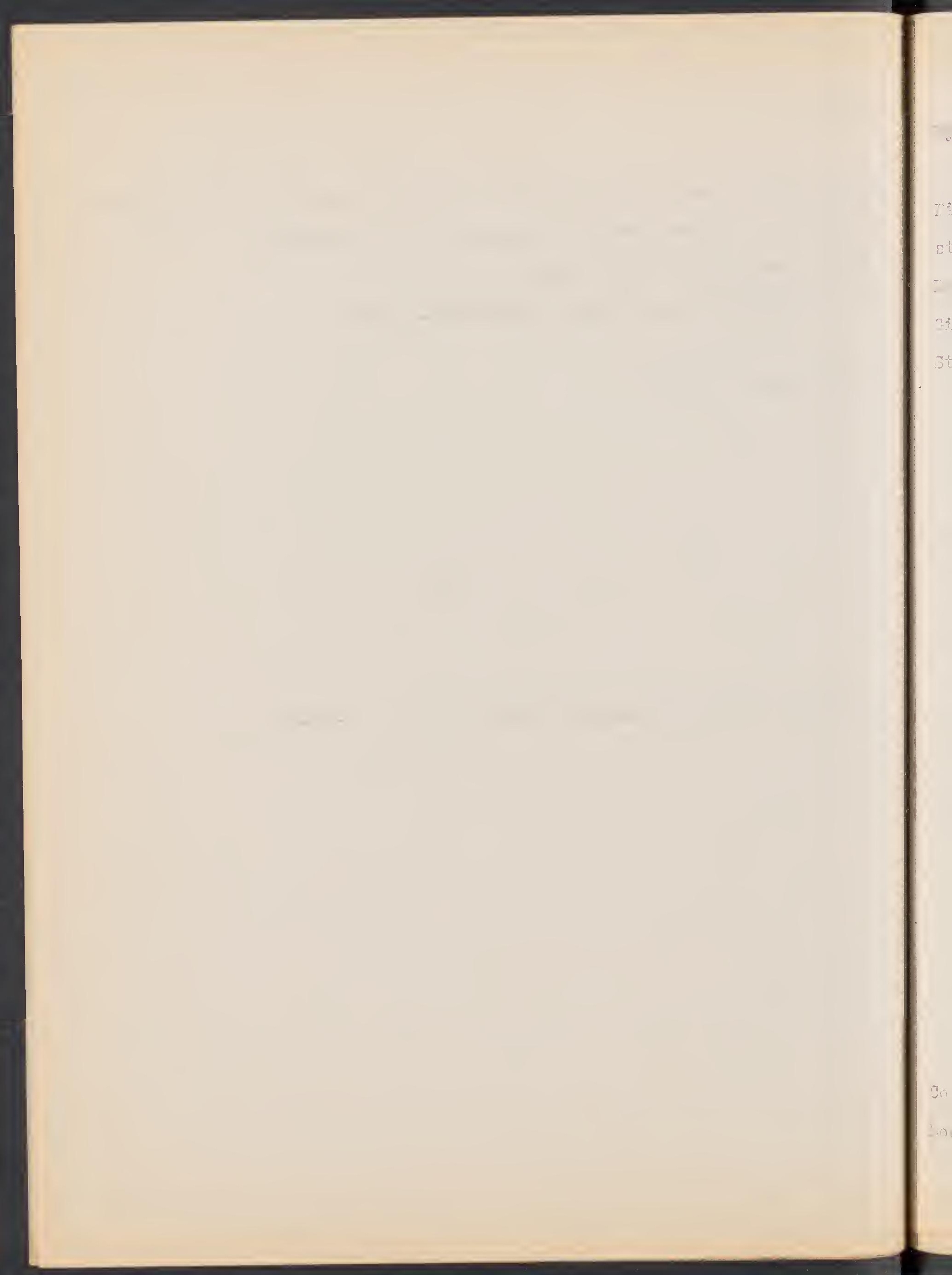




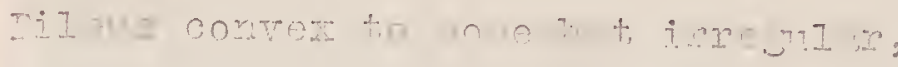

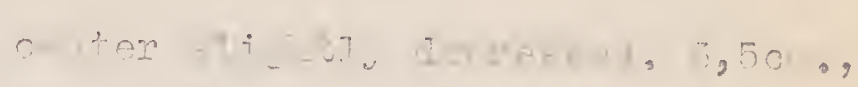

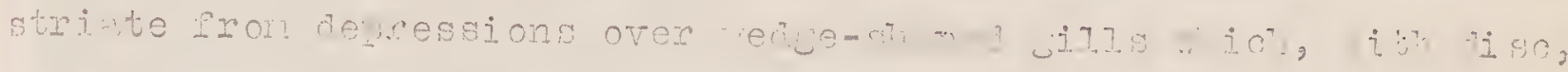

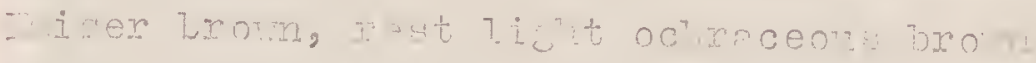

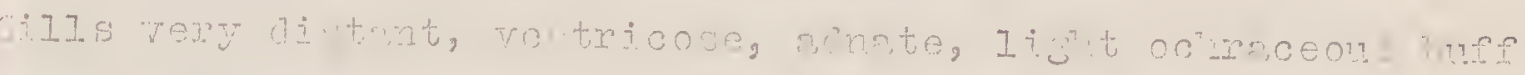

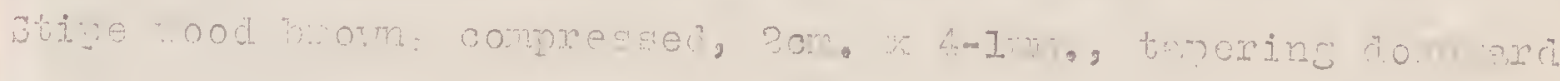

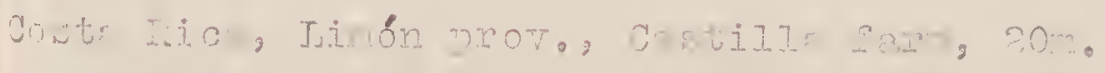



248

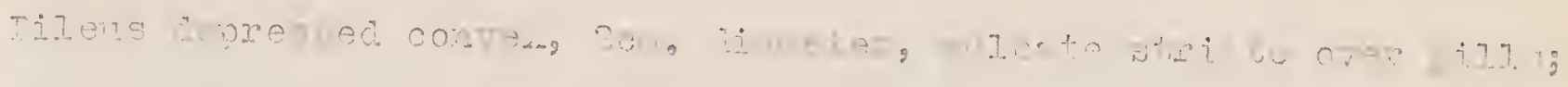

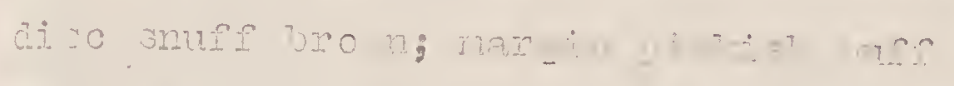

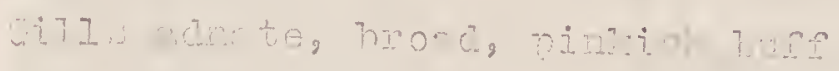

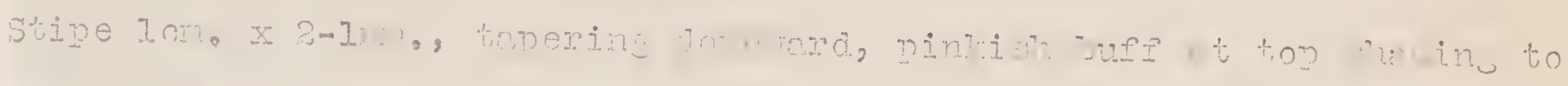

and se?

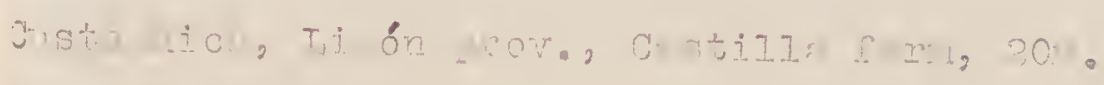

$7-7=-36$

node $=00 y$ 

Jeeria

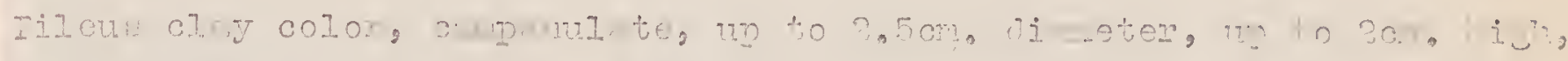

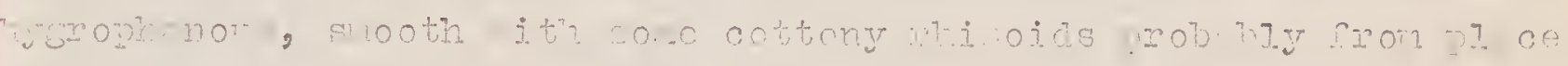

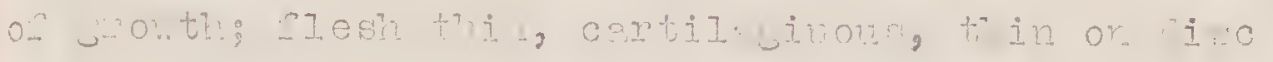

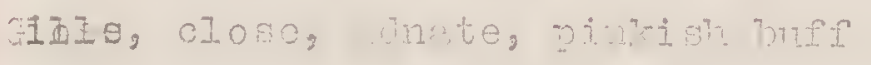

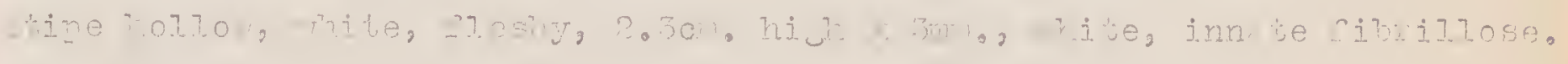

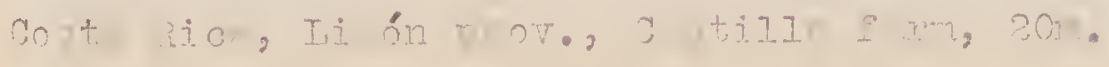

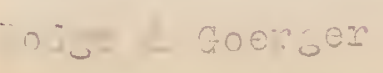



isen

Co.I.1.7

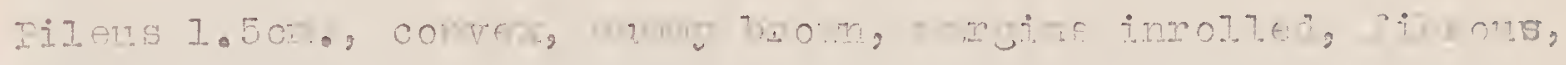

the contose

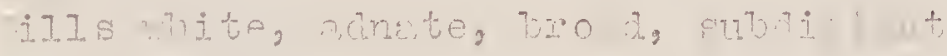

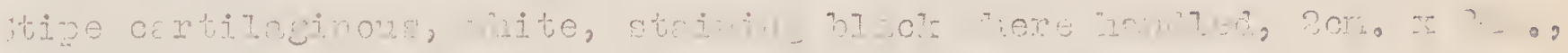
Simore

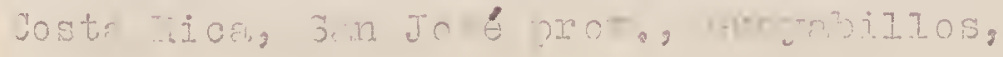



Teropia te

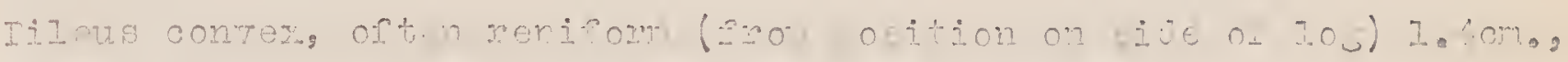

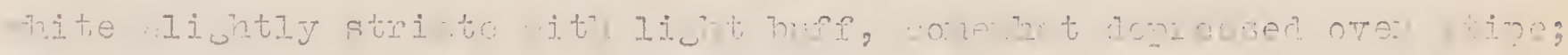
rin eren

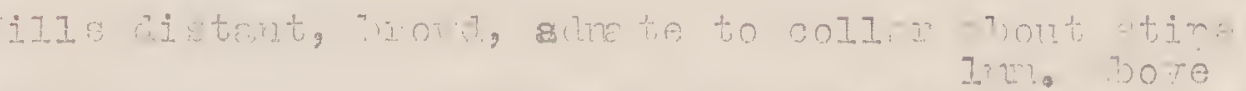

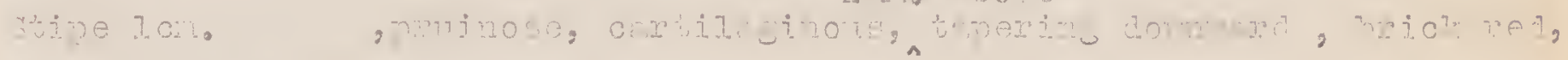

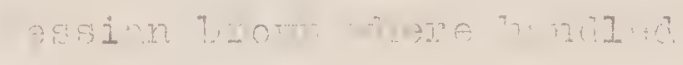

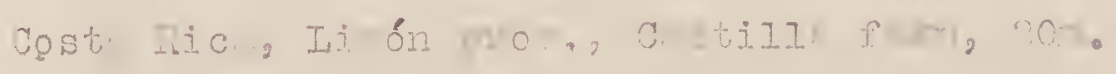
$7-n-10$ -ocice - songer 


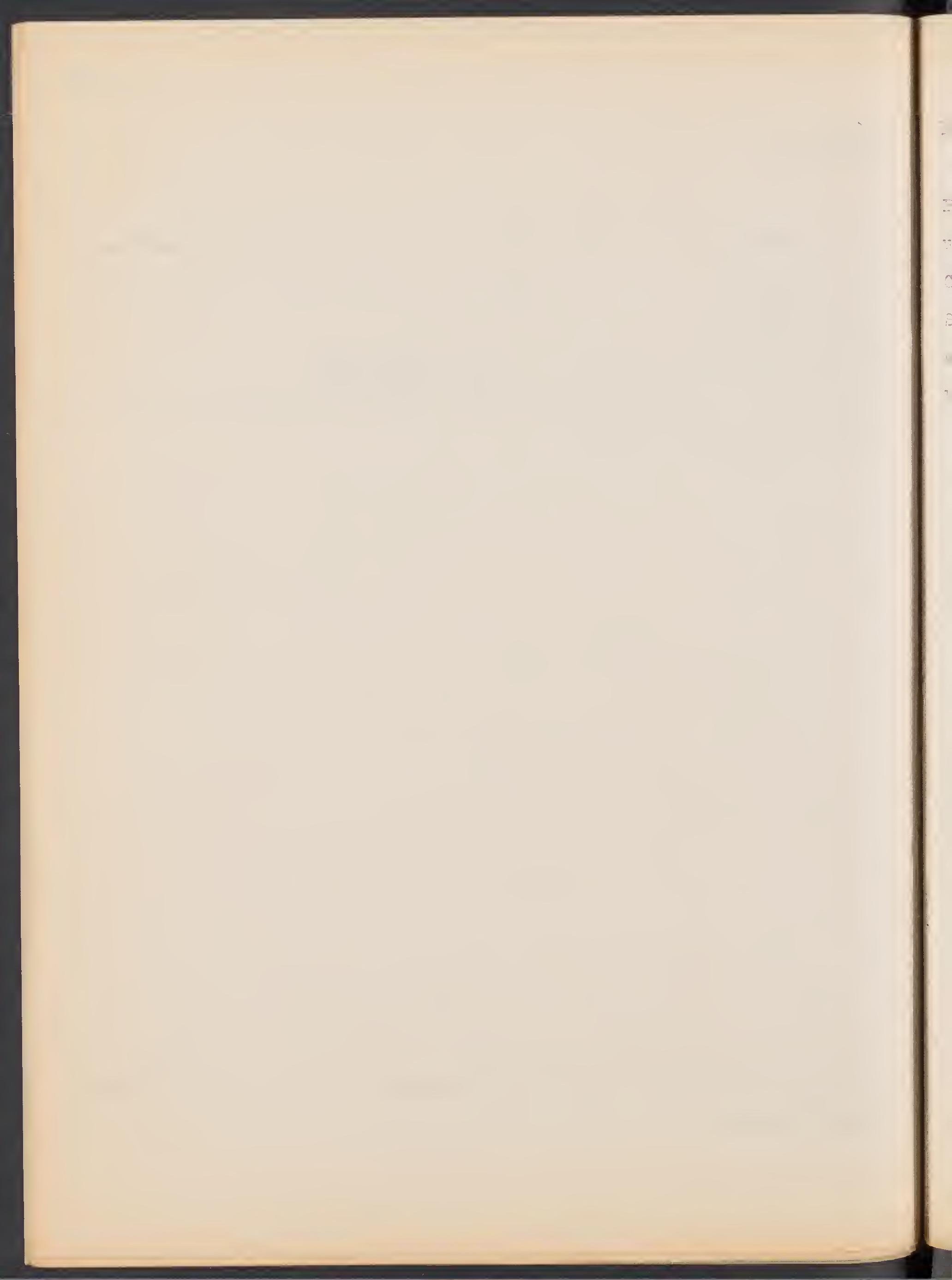


259

isosn:-

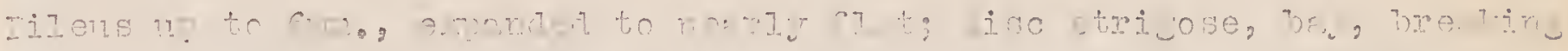

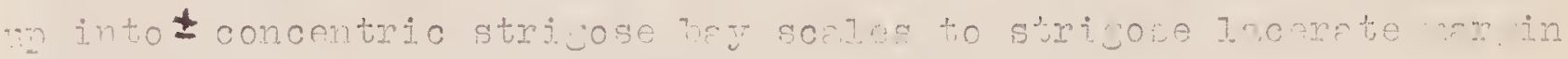

Nills rree, tontricose, n'rrot, olofa, whe tollow

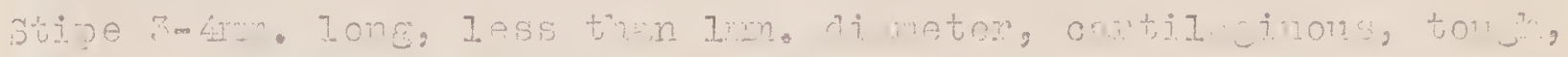

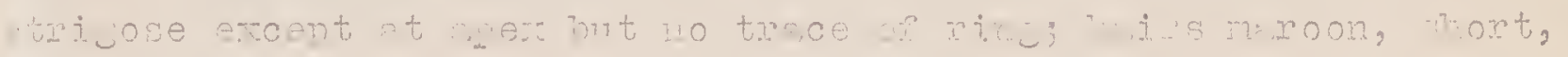

Tolo, the soatteren r ba

On $\operatorname{Senre]}$

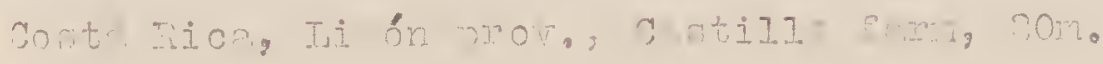

$\eta-28-6$

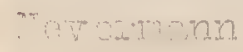


Coprinus comatus

Pileus $6 \mathrm{~cm}$. diam. $20 \mathrm{~cm}$. tall, white, lacerate, squamose, lower $4 \mathrm{~cm}$. striate; stipe white, smooth, silky, $37 \mathrm{~cm}$. \# $2 \mathrm{~cm}$ diameter.

Roadside between

Specimens examined:

Costa Rica, San Jose, between San Miguel \& San Isidro de Cononado,

$$
\text { Alt. } 1800 \mathrm{~m} \text {. Dodge } 4953
$$



coprinus

Pileus campanulate, striate, fibrillose, white when young becomine isabelline on disc, marein white, darkening as autolysis beeins $3 \mathrm{~cm}$ diam, $2 \mathrm{~cm}$ tall; gij.ls adnexed/ broad, crowded black, stipe fragile up to $11 \mathrm{~cm} \times 4 \mathrm{~mm}$, shite shining

Costa Rica: Puntarenas, Osa, near Rio Terrones $2-5 \mathrm{~m}$ tha Dodee 7725 

Coprinus

Pileus campanulate, $4 \mathrm{~cm}$ hieh, $5 \mathrm{~cm}$ diam, striate, hygrophanous isabeline; Eills crowded, broad, free, black; stipe whjte $15 \mathrm{~cm}$ $\times 5 \mathrm{~mm}$

Costa Rica: Puntarenas:0sa near Rio Terrones 20-30 m, Dode 7661 


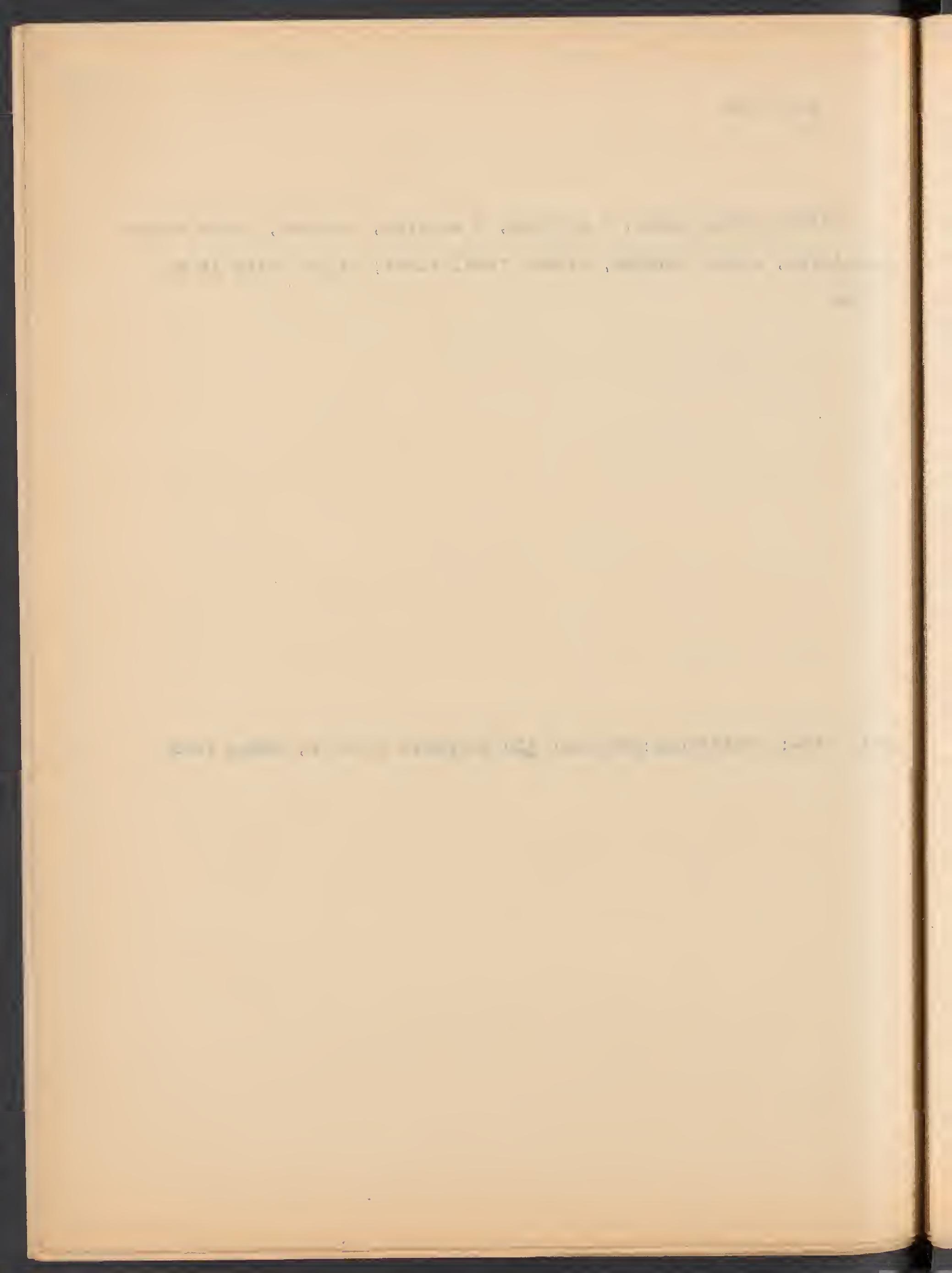


Coprinus

Plleus ovold to campanulate; universal vell not seen;

1 cm. either diameter, chamois or Lighter;gilis crowded, white, becoming black; stipe fragile, slender white, shining $3 \mathrm{~cm}$. \# $2.5 \mathrm{~mm}$.

Mossy log in potrero.

Specimens examined:

Costa Rica, San Jose, Rancho Redondo, Alt. 2160-2180 m. Dodge \& Thomas 5059 


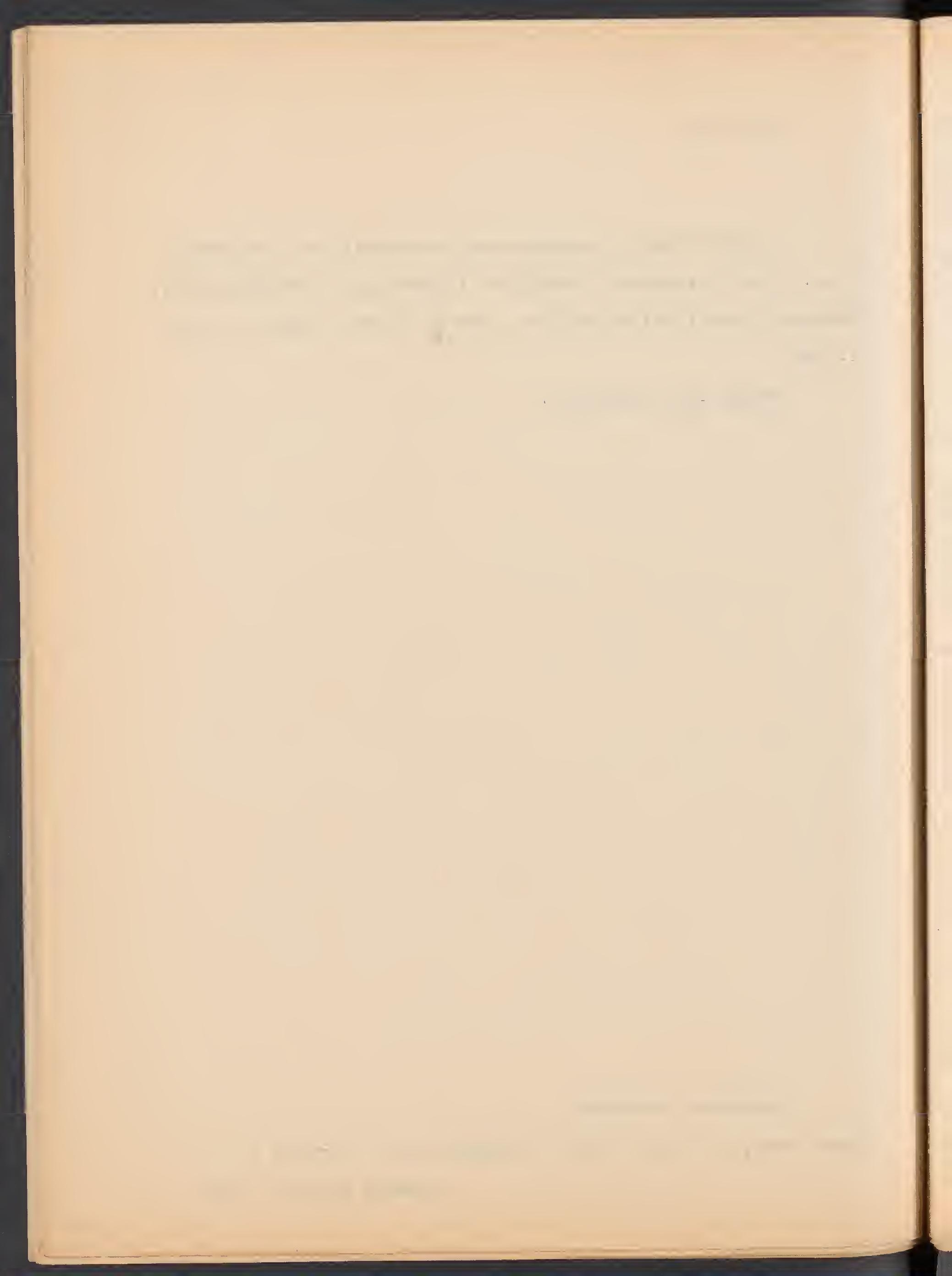


Coprinus

Plleus campanulate to expanded, covered with floccose, furturaceous universal veil, which quickly rubs off, leaving smooth, striate plieus with Dresden brwon disc, shadirg to avellaneous; gills nearly free, crowded, white, becoming black; stipe fragile, white, variable in length with agee of plant. Logs an poteero $S$. of house.

Specimens examined:

Costa Rica, San Jose, Rancho Redolndo, Alt. 2160-2180m.

Dodge \& Thomas 5060 


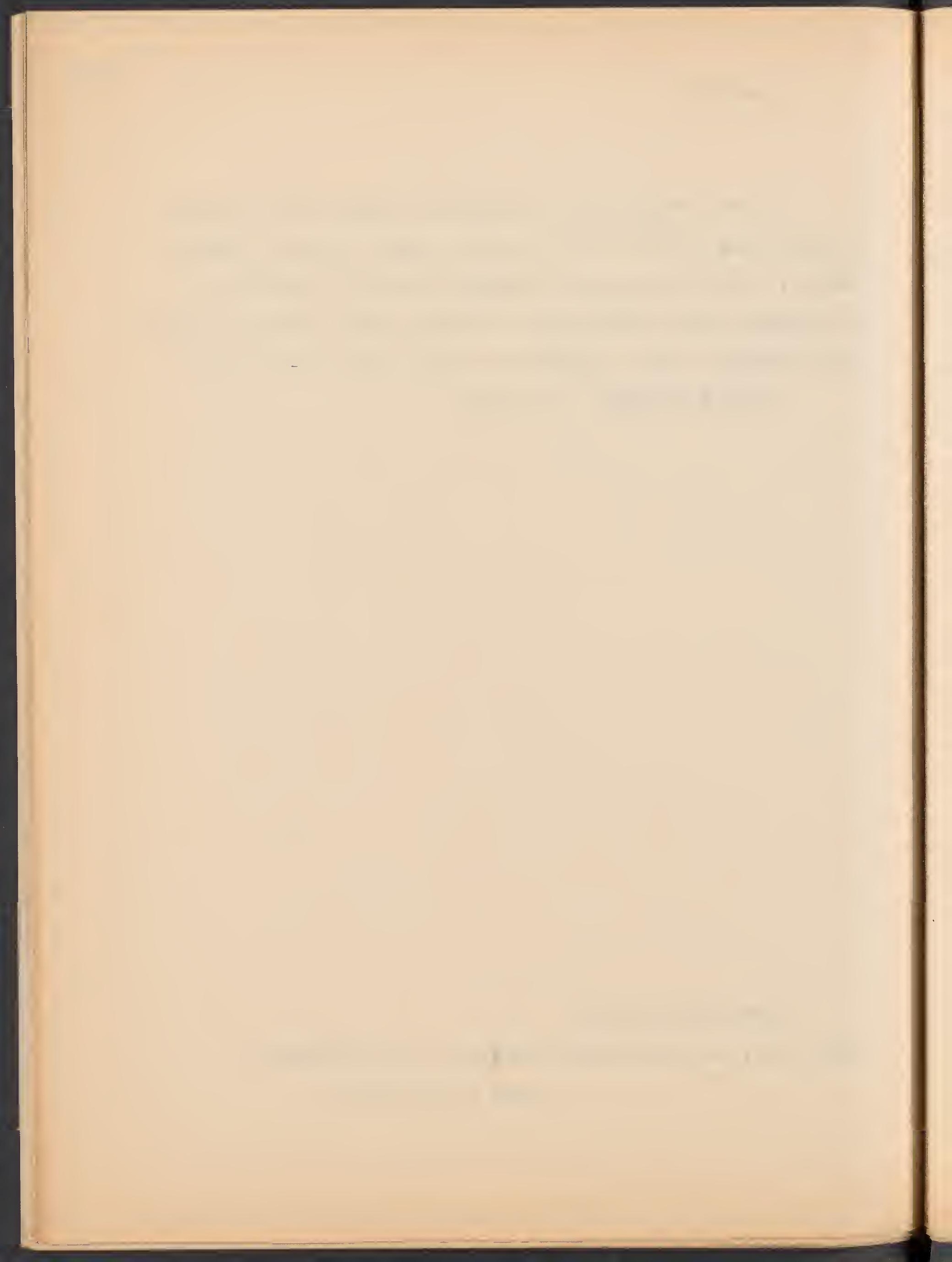


Coprinueoprinus

Pileus campanulate with heavy brwon tomentum, often arranged in pyramidal warts, rubbing off, leaving smooth or pruinose, shading from buckthorn-brown to warm -buff on the striate, thin margin; $1.5 \mathrm{~cm}$. diameter $1.8 \mathrm{~cm}$. tall; flesh very thin; gills crowded, nearly free; wide;margin of pileus breaking awy from margin of butb by elongation of stipe, which is fragile, $2 \mathrm{~cm}$. Iong, $4 \mathrm{~mm}$. diameter, white, silky.

on 108 in potrero 5 . of house.

Specimens examined:

Costa Rica, San Jose, Rancho Redoddo, Alt. 2160-2180m. Dodge \& Thomas 5109 


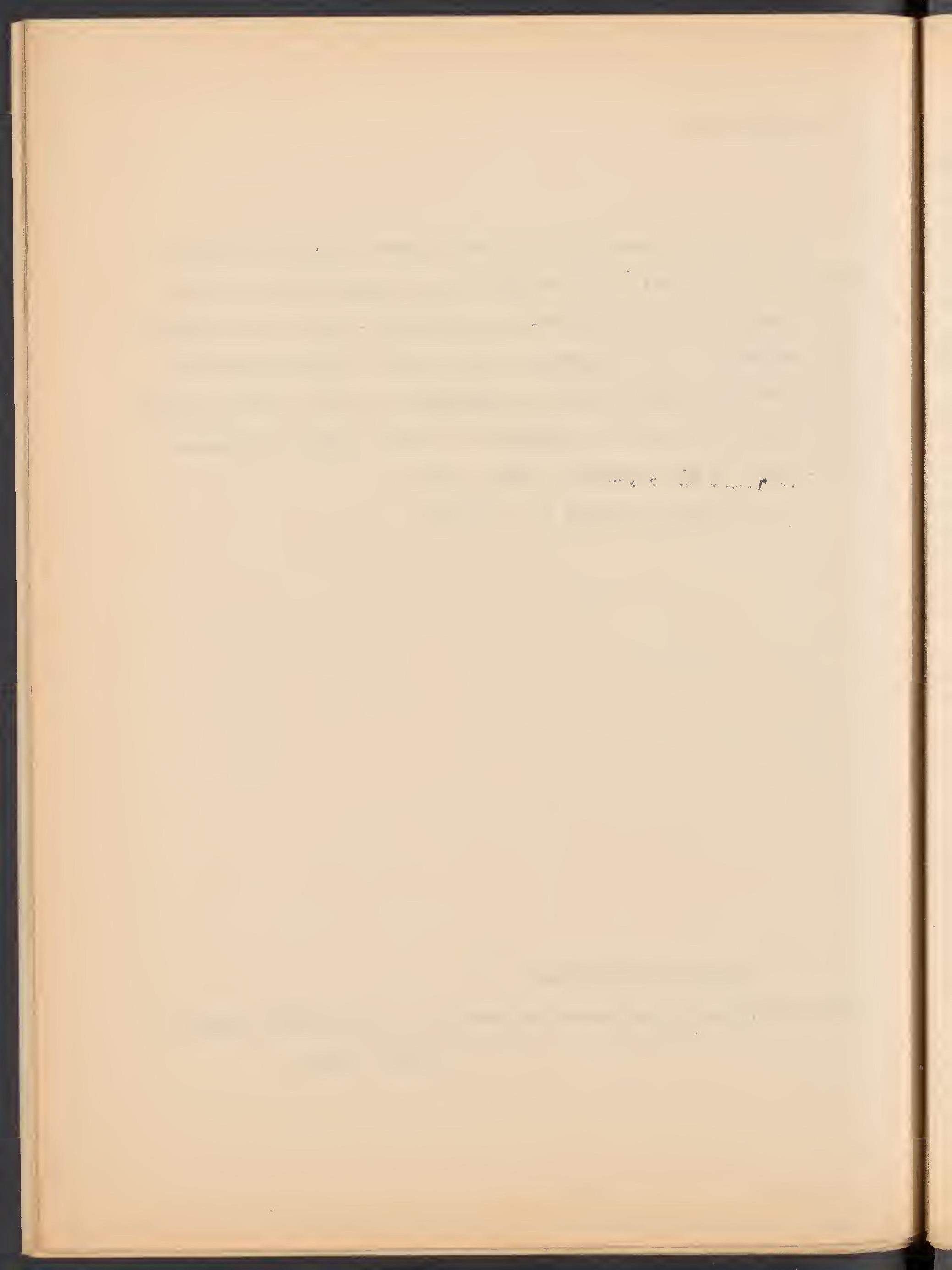


Coprinus

Pileus campanulate, $3.5 \times 3 \mathrm{~cm}$ thin, striate, pruinose except on disc which is cinnamon brown shading to ochraceous tawny with still lighter margin, very fragile; gills free broad, crowded edges pruinose, sti pe white $6 \mathrm{~cm}$ hollow, very fragile, silky fibrous

dead stumps, quite common at Rancho Redondo

Specimen examined:

Costa Rica: San Jose, Rancho Redondo 2300 m Dodge\& Thomas 5388 


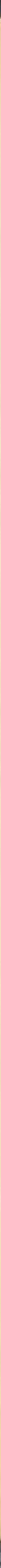




\section{Cortinarius}

Pileus convex, viscid, twwny to russet, darker when moist, margin appendicula te with fragments of cortina especially when young; flesh white, thin except on disc; Eills broad, close, white becoming brownish, adnexed; stipe cartilaginous hollow, white stained red brown by remains of cortina $1.5^{\mathrm{cm}} \times 5 \mathrm{~mm}$ above taperine to $3 \mathrm{~mm}$ of Nancoria $5 / 64$ 
'z98 oprquzdr

oty ezson "asor uns. 
Cortinarius

Pileus hemispheric, conic expanded, convex, with broad umbo, innate fibrillose scaly, moist but not viscid, $2.5 \mathrm{~cm}$ diam, amber brown shading to raw sienna, flesh thick on disc, watersoaked; with

gills adnate very narrow, close, raw sienna; stipe fleshycartilaginous rind: cortina present when youne, eranescent learing a few fibrils at point of attachment of $\varepsilon i l l s$, surface fibrous, sliehtly darkerwhere handled, raw sienna.

Mossy log.

Specimens examined

Costa Rica: San Jose, Rancho Redondo, $2400 \mathrm{~m}$, Dodge \& Thomas 5022 


$$
\ldots+\infty+\cdots+\infty+\infty
$$


262

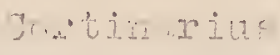

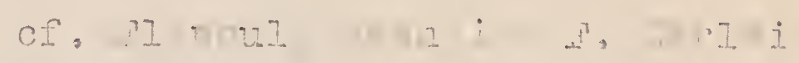

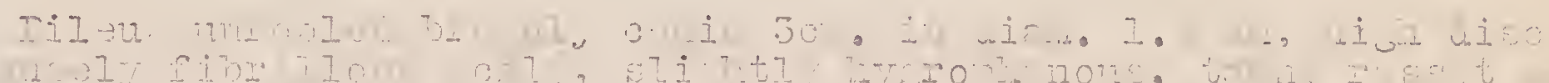

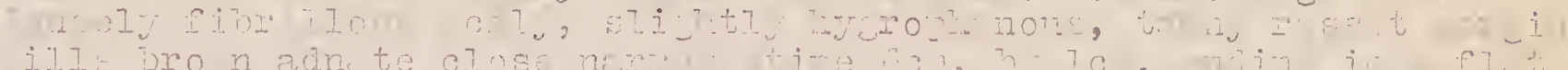

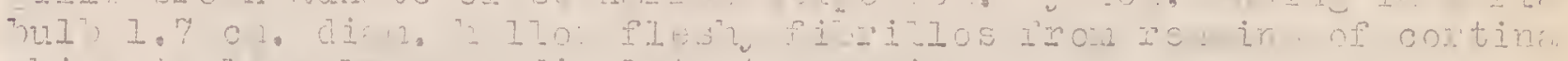

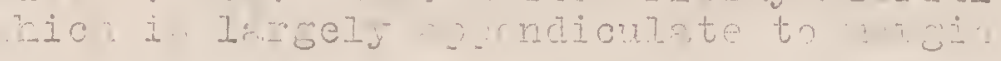

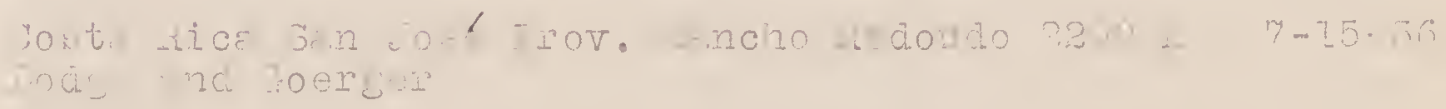


Cortinarius
of Collfiatemipes group if spones white

Pileus hemispheric (not fully expanded\%) about $1 \mathrm{~cm}$ diam xanthine orange, slightly lighter toward the margin wi th 5-6 sided truncated pyramidal warts (B. variegatus like);

fle sh thick, watery, eills apricot yellow, broad, close, adnexed edges lighter, pruinose, crenate, serrate, cortina present, about the color of the gills; stipe antique brown on handling, fibrillose scaly squamose, apricot yellow, cartilaginous hollow, $17 \times 2.5 \mathrm{~mm}$ bank by roadside

SPecimens examined:

Costa Rica: San Jose, Rancho Redondo,2300 m, Dodge \&Thomas 5114 


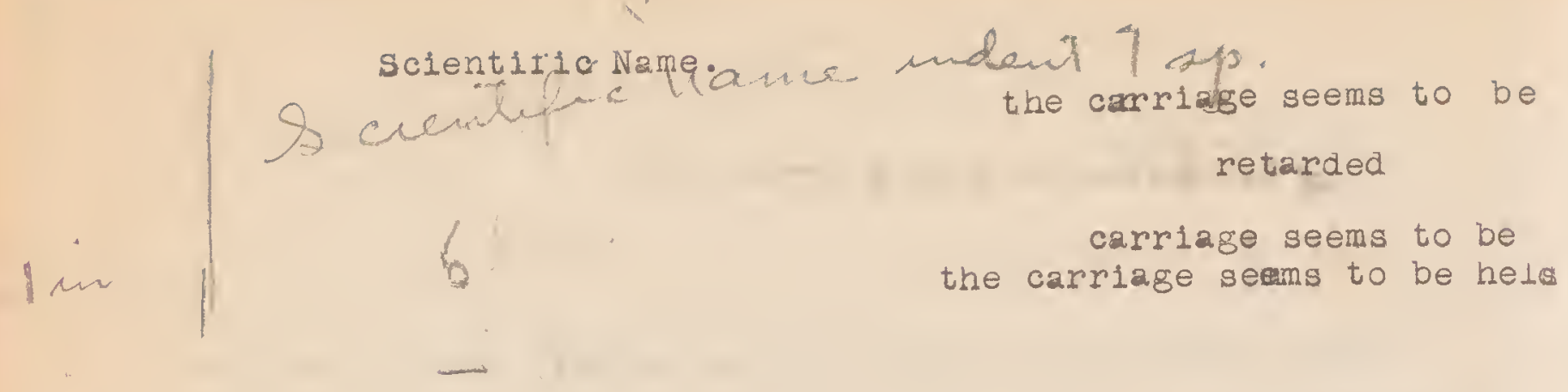

Specimens' examined:

alt.

Costa Rica: San Jose, Rancho Redondo, Dodge \& Thomas 5262 
crepidotus of cacophyllus

Pileus tawny, scaly above.

Rotten 108 .

Specimens examined:

Costa Kica, San Jose, Rancho Redonao, Alt.2250 m. Doage ana Thomas $\quad 5124$ 
Creplaótus

Pileus applanate to depressed, flabeliform to dimidiate by coalescence, blackening above, buckthorn- brown, hygrophanous; gills thick, crispea interveinea cinnamon buft, decurrent along polnt of attachment.

Dead Log.

\section{Specimens exmanlea:}

Costa Rica, San Jose, Rancho Reaonao, Alt.2y-2400m. Dodge ana Thomas 5049 

Crepidotus

Pileus up to $6 \mathrm{~cm}$. partly resupinate to point of attachment above and near center but plieus split up trunderside as inverted otides, viscid, buff near point of attachment, otherwise white, reticulate with water-soaked veins; 8111 s close to crowded, wide, edges 1 Loccose with remain s of partial veil.

Dead stump in potrero.

Specimens examined:

Costa Rica, San Jose, Rancho Redoâdo, Alt. 2600m. Dodge \& Thomas 


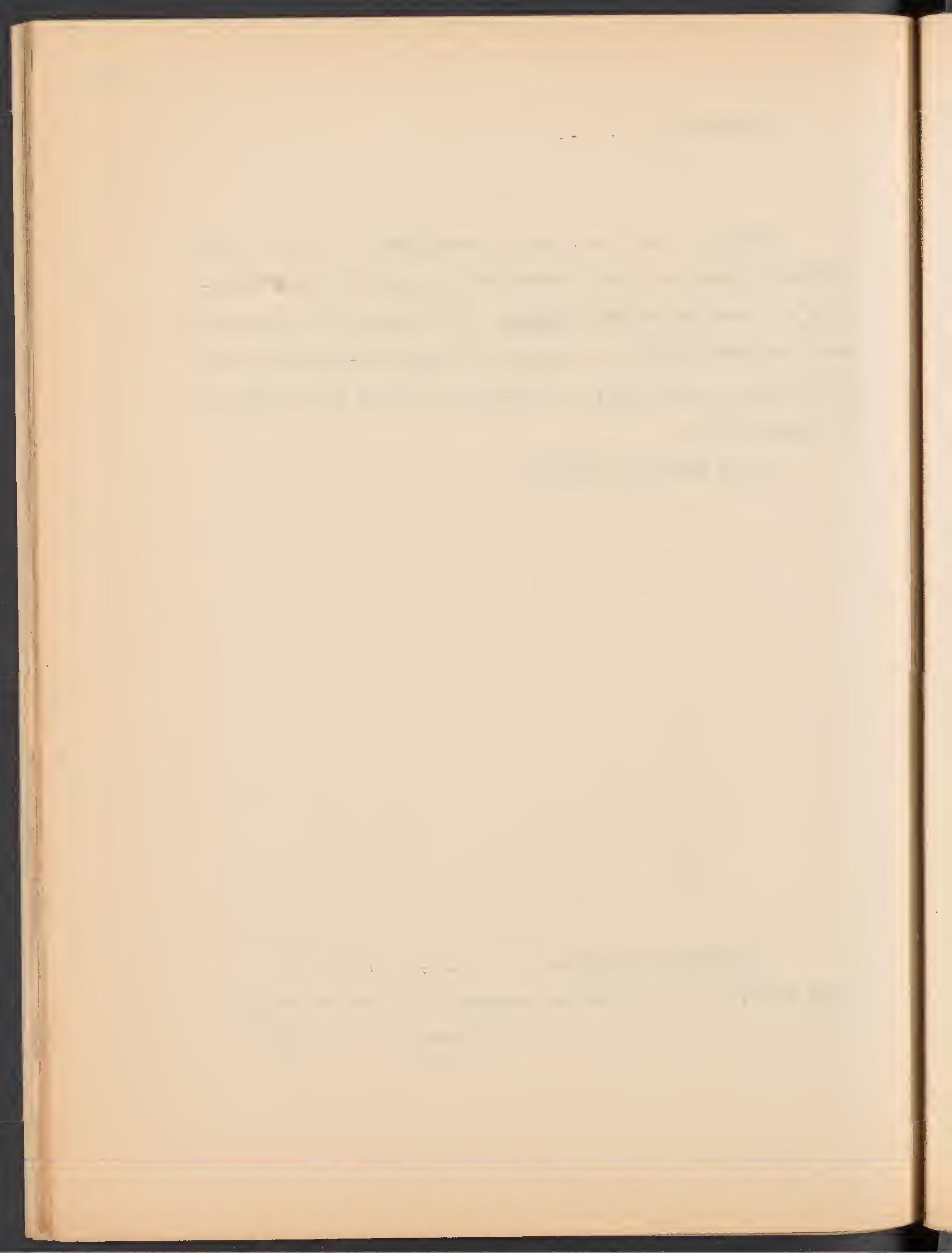


Crcpjdocrepldotus near $C$. fumosifollus?

pileus subdimidiate up to $2 \mathrm{~cm}$. diam., white above, brown below, surtice smooth with very small pyramidal brown groups of fibres; lamellae adnate to point of attachment. Dead stub on living tree in forest.

Specimens examined:

Costa Rica, Cartago, La Carpintera, Alt. 1500m. Dodge \& Thomas

? Sumon: Waldeck $40 \mathrm{~m}$ Dodge 7318 
Crepidotus calolepidoides group

Dead wood in potrero south of house

Specimens examined:

Costa Rica, San Jose, Rancho Redondo, Alt. 216062180 , Dodge\& Thomas 5123 

Plieus dimidiate, $6 \times 4 \mathrm{~cm}$. expanded, surface, fibrillose, scaly, subhygrophanus, margin watery, disc snuff-brown, shading to cream-buff, dotted with snuff-brown scales, gsils decurrent along point of attachment, crowded, subventricose,, snuff-brown to bister.

On bark of living Eucalyptus sp. Garden

Specimens examined.:

Costa Rica, San Jose, Rancho Redondo, Alt. $2150 \mathrm{~m}$. Dodge 5129 



\section{Crepidotus}

Pileus flabelliform, $2.5 \times 2 \mathrm{~cm}$, flat, clore brown, dull, margin faintly striate; gills close, medium width, decurrent along point of attachment, olire brown; flesh tery thin. Mossy long in potrero south of house

sp̄ecimens examined

Costa Rica: SanJose, Rancho Redondo, 2160-2180 m Dodee \& Thomas 5064 


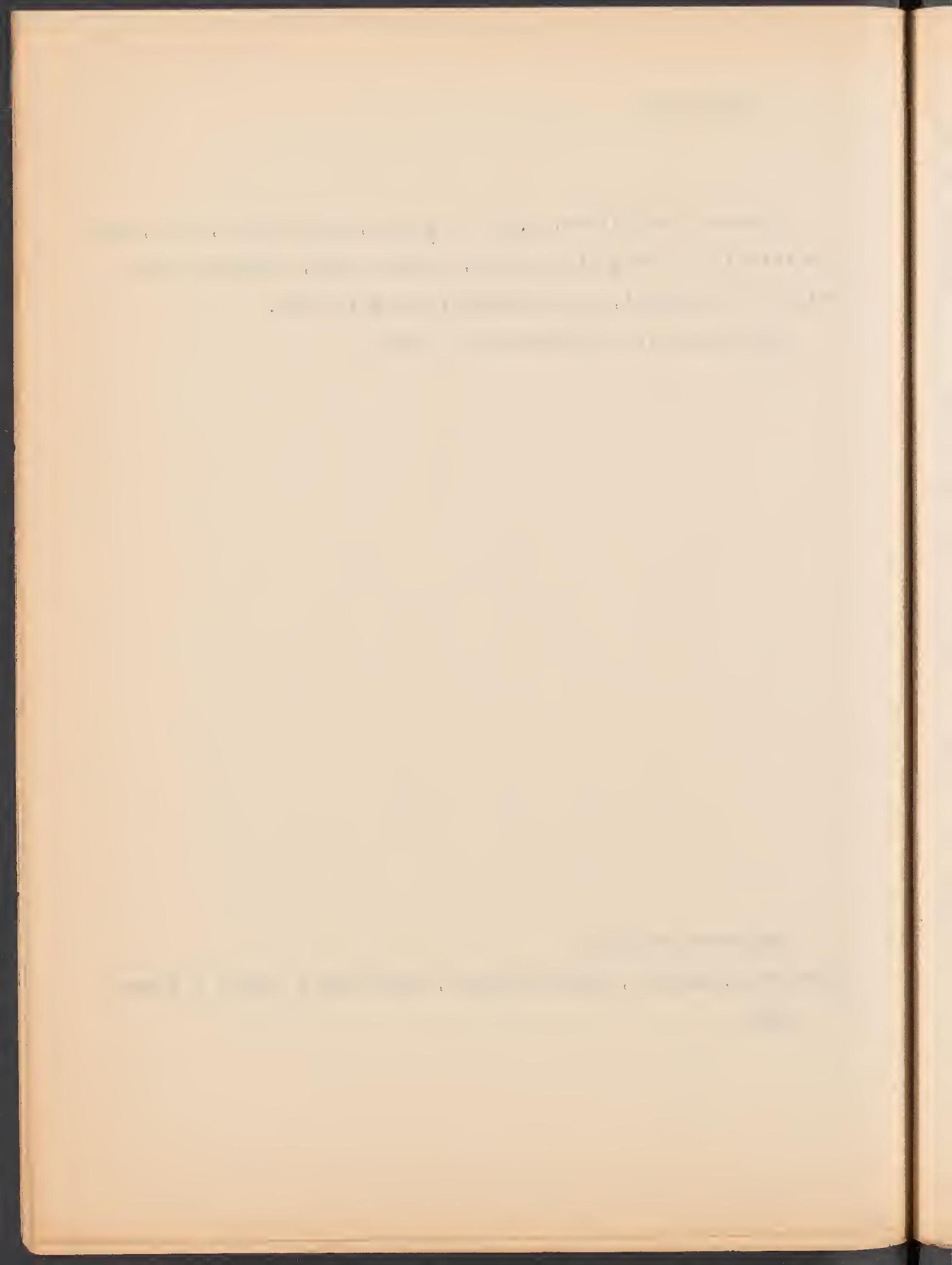


Crepidotus

pileus $2.5 \mathrm{~cm}$. diameter, flabeliform to subdimidiate, flat to slightly convex, clay color; gills decurrent alons point of attachment, cream-buff or darker, close, ventricose, attachment lateral with no trace of atipe.

on stump by roadside.

Specimens examined.

Costa Rica, San Jose, Rancho Redondo, Alt. $2270 \mathrm{~m}$. , Dodge \& Thomas 4981 

Crepidotus

Pileus flabelifiorm to somewhat aimiaiate, expandea surface, smooth, hygrophanous, Ilght-pinkish-cinnamon, substriate; gills concolor, adnate to olive buff stipe which appears IlabelIlform below up to $5 \mathrm{~mm}$. Iong, diameter wholly lateral. Mossy bank by roadside.

Specimens examined;

Costa Rica, San Jose, Rancho Redondo, Alt.2500m. Doage \& Thomas 4982 


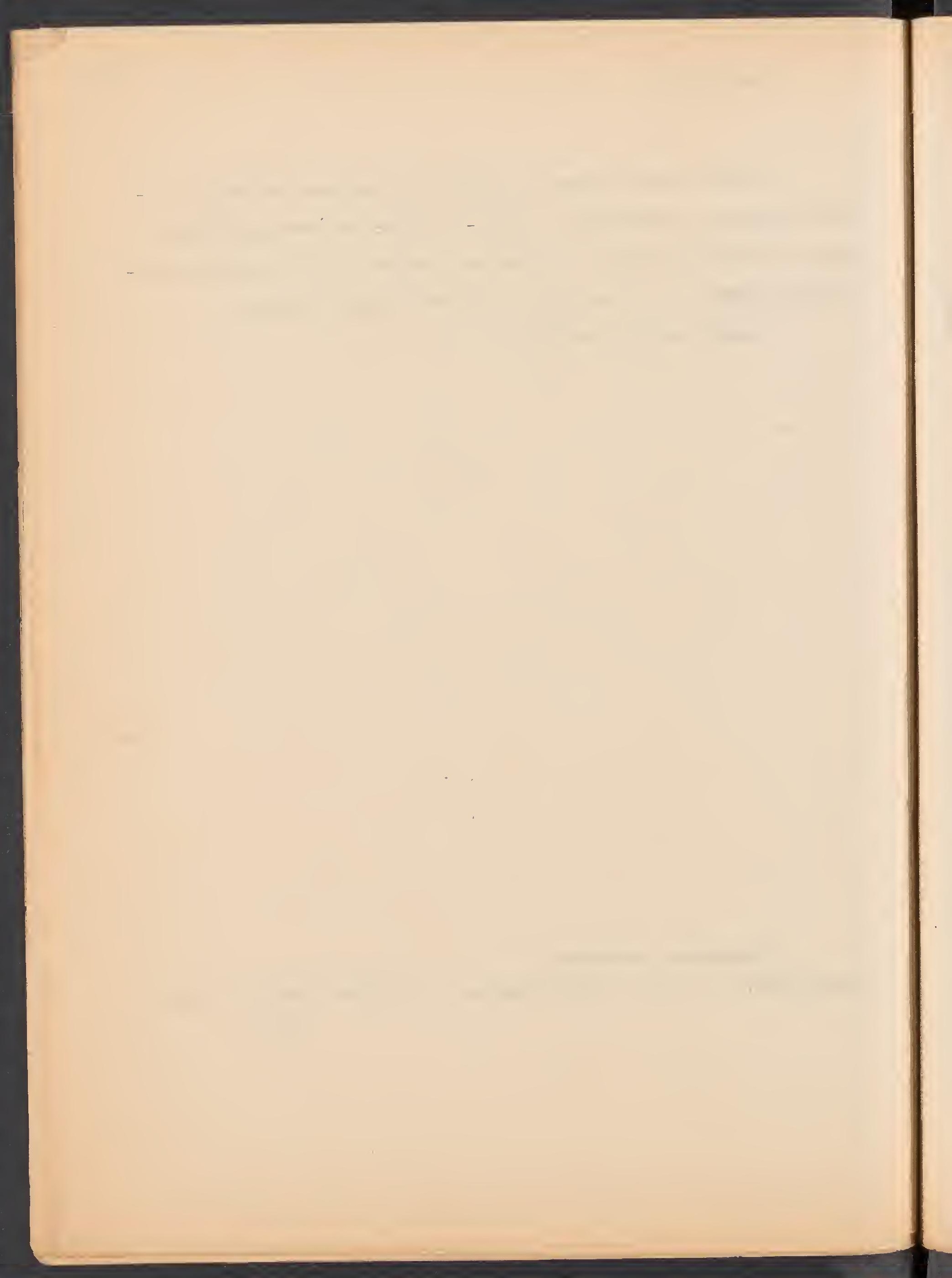


Crepidotus

Pileus flabelliform, slightly conrex, tomentose with fascicles of auburn fibres on moist licht ochraceous salmon surface; gills adnate to piint of attachment, tilleul buff, rentricose, edges slightly wary; attachment concolor, $5 \mathrm{~mm}$ lone $3 \mathrm{~mm}$ diameter

specimexs exaruined

Costa Rj.ca: San Jose, Rancho Redondo 2180-2350 m Dodge \& Thomas 5002 


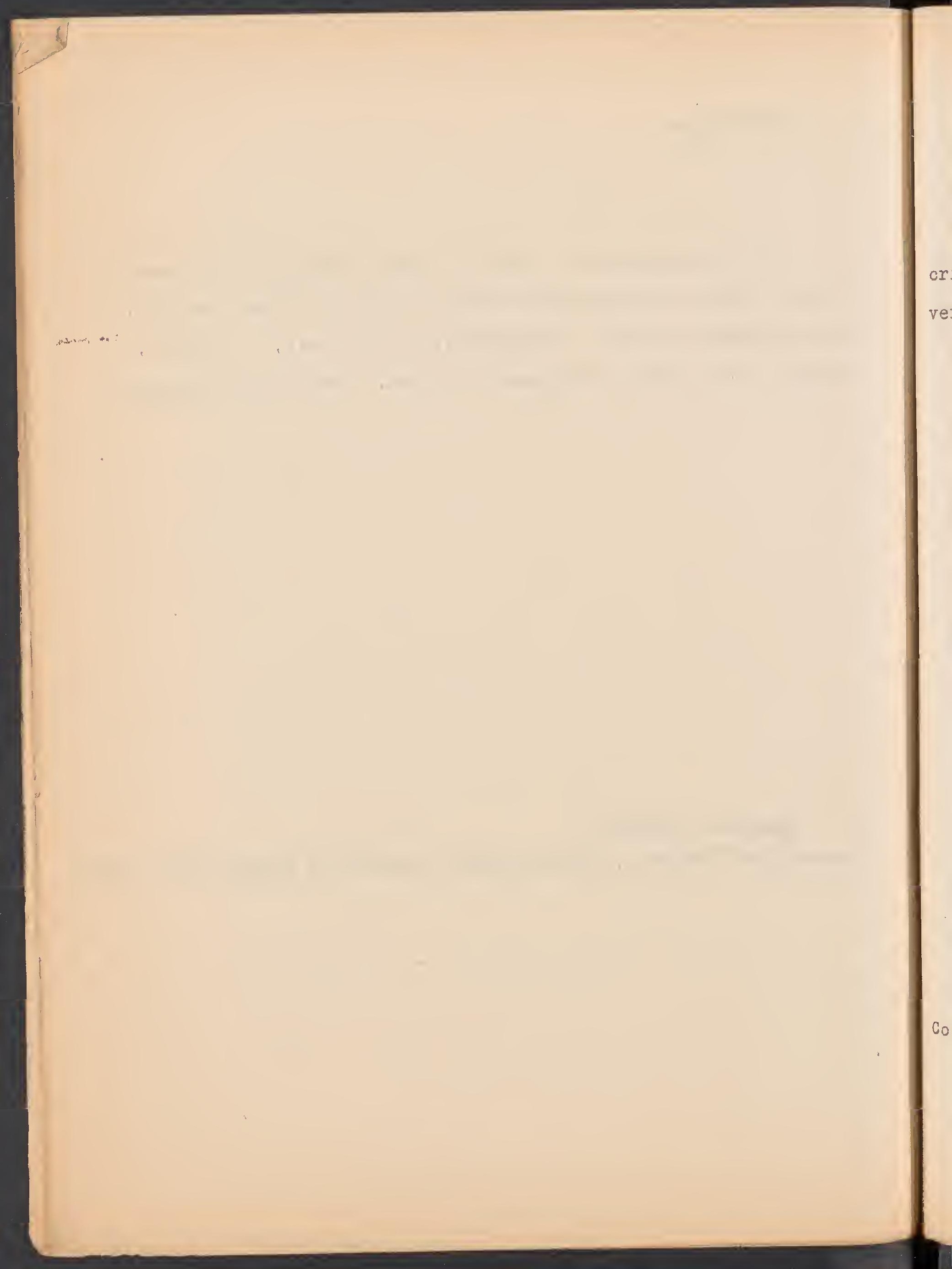




\section{Crepidotus}

Pileus minutely tomentose, ochraceous-buff, $5 \# 3.5 \mathrm{~cm}$. margin crisped, striate; eills amber-brown, adnate, closem,narrow; stipe ven very short.

Decaying log in cassisva patch.

Specimens examined:

Costa Rica, Limon, Iivineston Farm, Alt.70-80 m.,Catt, Dodé \& Thomas 
Crepidotus $\mathrm{n}$ sp.

Pilei close, 5-10 mm. Ejabrous, avellaneous or likhter;

very thin;context; eills broad, distant, becoming cinnamon, adnate to slender; short lateral stipe.

Bark of stump.

Sipecinens extmined:

Specimens examined:

Costa Rica, Limon, above Rio Siquirres, Alt.200-300m., Dodge \& Catt, \& Thomas 5554 


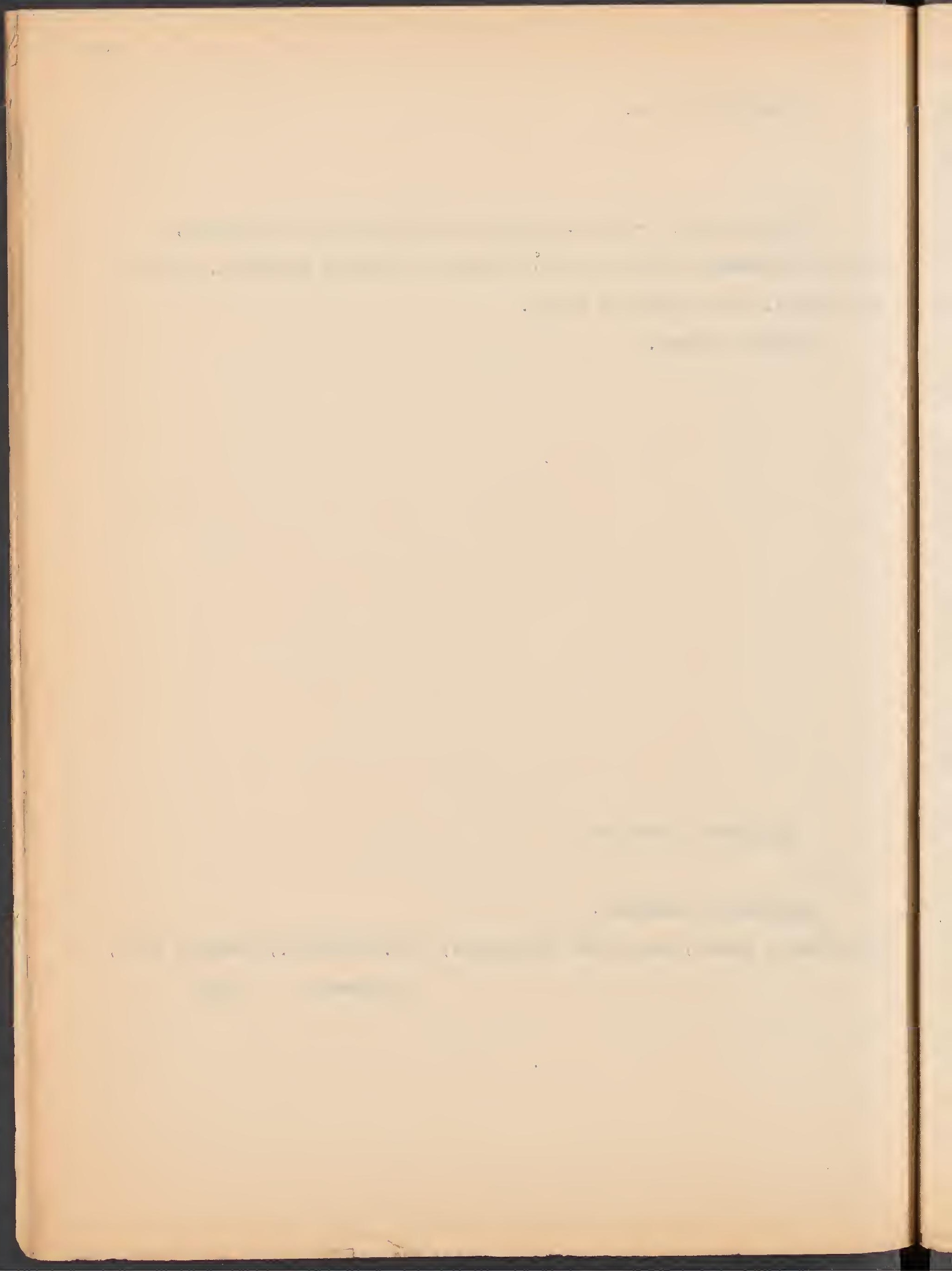


Crepidotis neas cuchonensin

Pilei crowded imbridute, sub conic, hycrodrknous, white above,

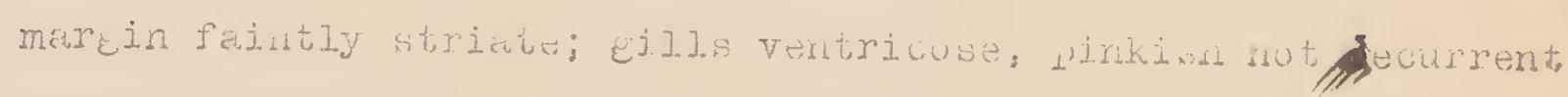
alone tiny itipe which is laterel ard 6 inm lond

on dead wood

\section{Sitecimerls exiwijned}

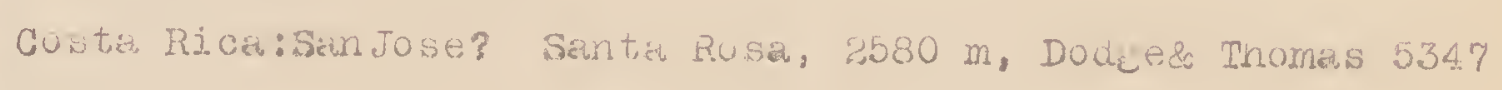


Deconice

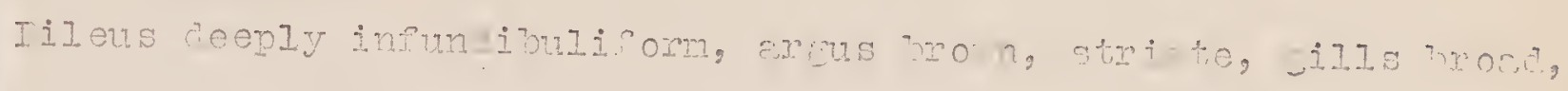
decurent, distaint, concolorors

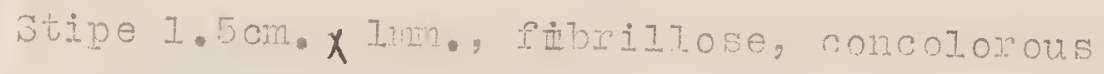

on cern roots

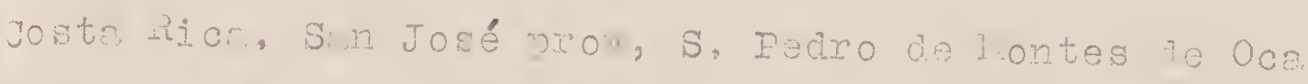
"everinann is Goerger 
Eccilia

Pileus very fragile with 3 splits to stipe, when collected, infundibuliform, $3 \mathrm{~cm}$.. white,disc, marein striate, pale ochraceou ous- salmon; gills broad, decurrent, subdistant, pink; stipe very fragile, white, cartilacinous, hollow, $2.5 \mathrm{~cm}$. \#2.5 mm.

Specimens examined:

Costa Rica, Limon, Rose Hill above Rio Pacuare, A.t. 70-120 m.. Catt, Dodge \& Thomas 5592 

Bccilia near Farlei

pileus umbonate, up to $3 \mathrm{~cm}$, marein inrolled, notstriate, disc ochraceous buff; $\varepsilon^{j . J . s}$ decurrent, subdjstant, broad, pinkish cinnamon stipe $3.5 \times 3 \mathrm{~mm}$, smooth, lieht ochraceous buff.

Costa Rica: Funtarenas, Osa, Cerro Guaca Dodge 7641. 


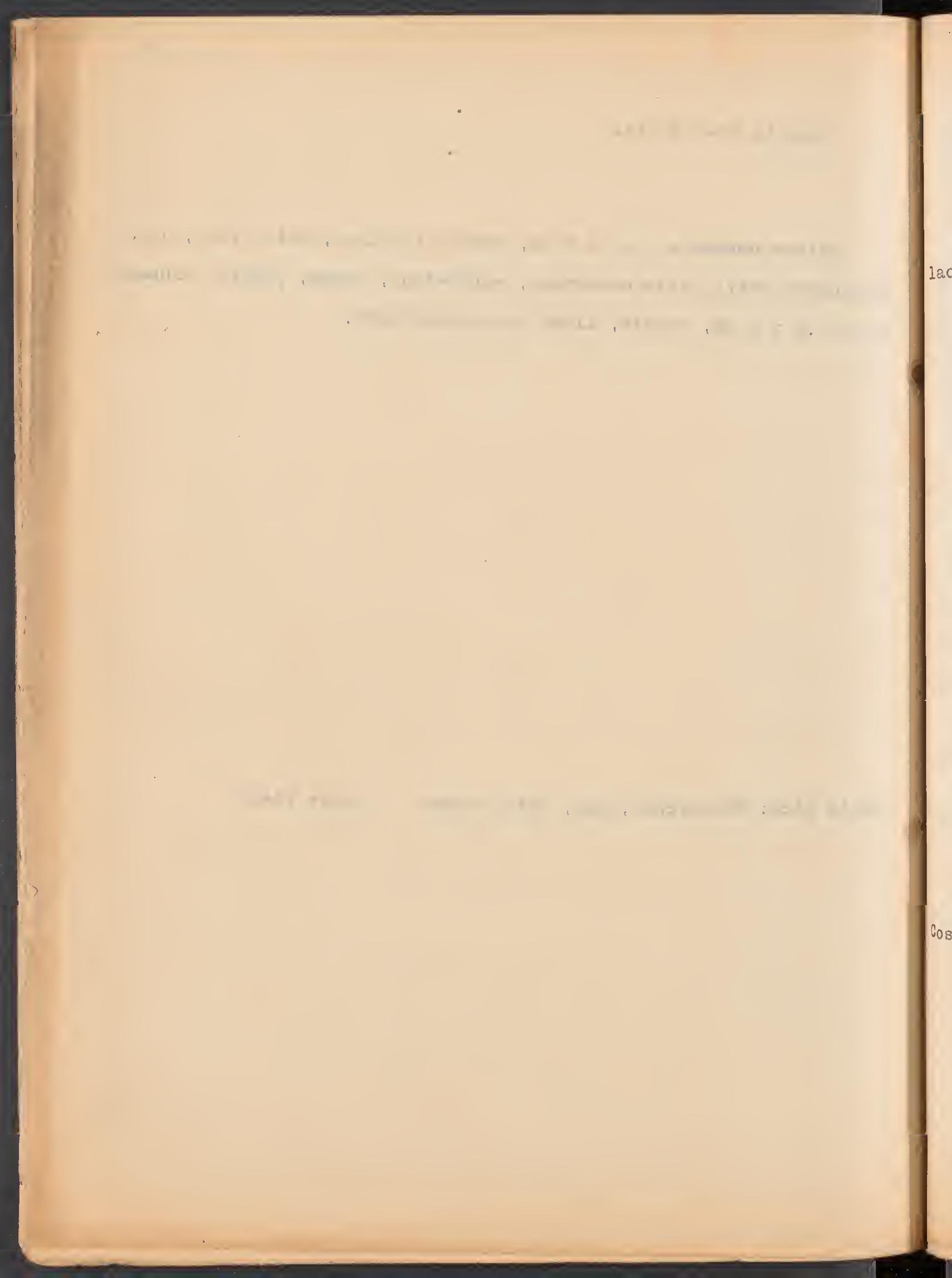


Favolus

Pileus dimidiate up to $3 \mathrm{~cm}$, not tessellate; stipe almost lacking.

Specimens examined:

Costa Rica, Limon, Siquirres South Farm, above Rio Siquirres, Alt. 70-200m, Catt, Dodge \& Thomas 5562 
Farolus $\mathrm{n}$ sp.

Pileus tomentose, 6 \# , wholly tesselate, margin thin, dentate or sublacerate, context membranous, except on disc, pinkishbuff to cinnamon-buff; tubes slightly decurrent, white edees, fimbriate, eroded; stipe short 7 \# $6 \mathrm{~mm}$. lateral, concolorous.

Specimens examined:

Costa Rica, Limon, Barnstorf Farm, Catt, Dodge, \& Thomas 5541 


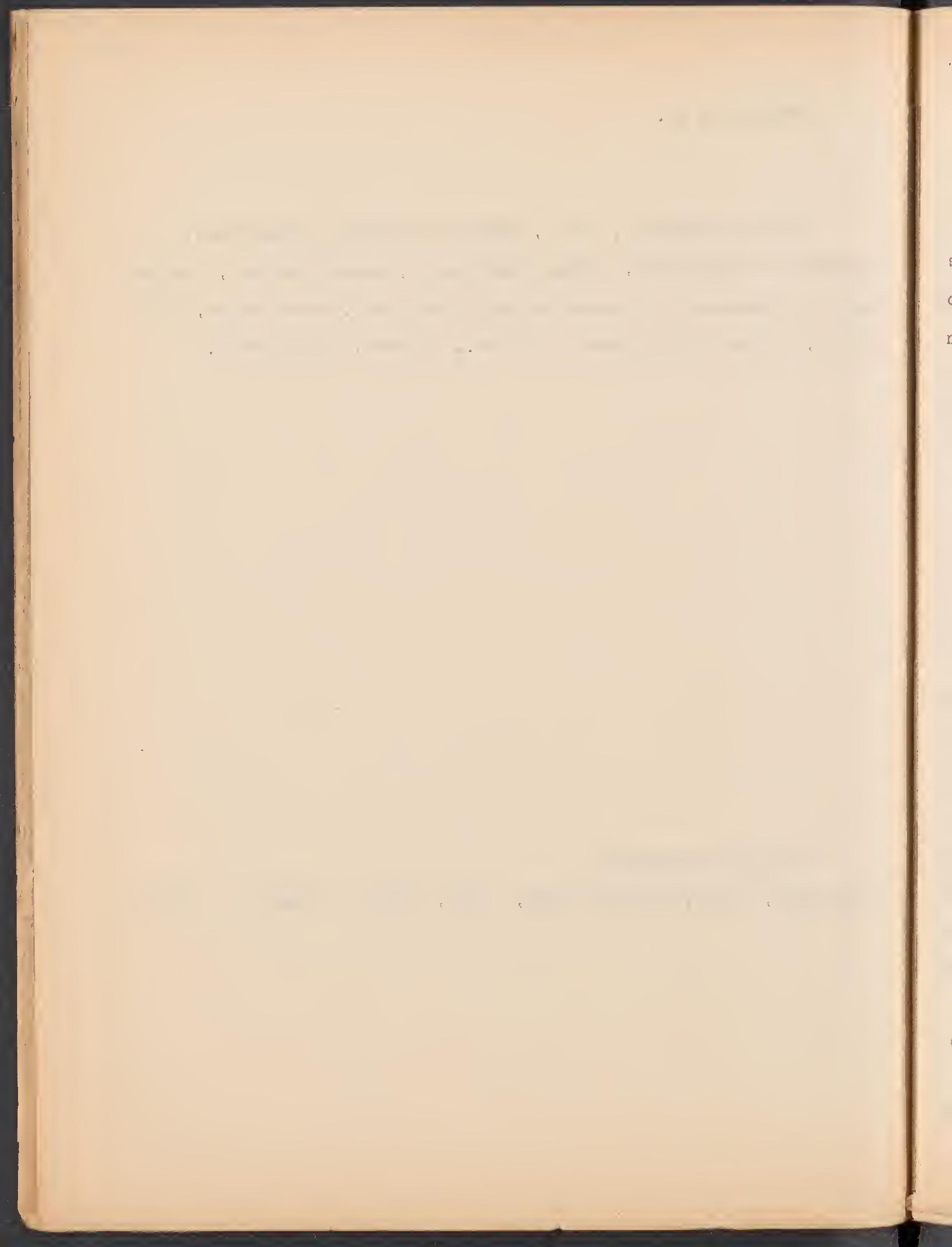


Favolus near renjformis.

Pileus $5 \mathrm{~cm}$, minutely tomentose, tesselate with tendency to split down; tubes as in Hiatula, context almost membranous; tubes decurrent on stipe, edges fimbriate; stipe 1.5 \# $4 \mathrm{~mm}$., white, tomentose below tubes.

Specimens examined:

Costa Rica, Limon, Barnstorf Farm, Gätt, Dodee, \&Thomas 5536 
fravolus near suocalerata ir ney.

Pileus $8 \mathrm{~cm}$. surface, tomentose, tessellate on marein, smooth on disc; stipe Jateral; tipbes some what decurrent, $2.5 \mathrm{~cm} \# 8 \mathrm{~mm}$., sponey, tomentose, below tubes.

Dead Iogs.

Specimans examined:

Costa Rica, Iimon, Barnstorf Farm, Catt, Dodue \& Thomas 5530 


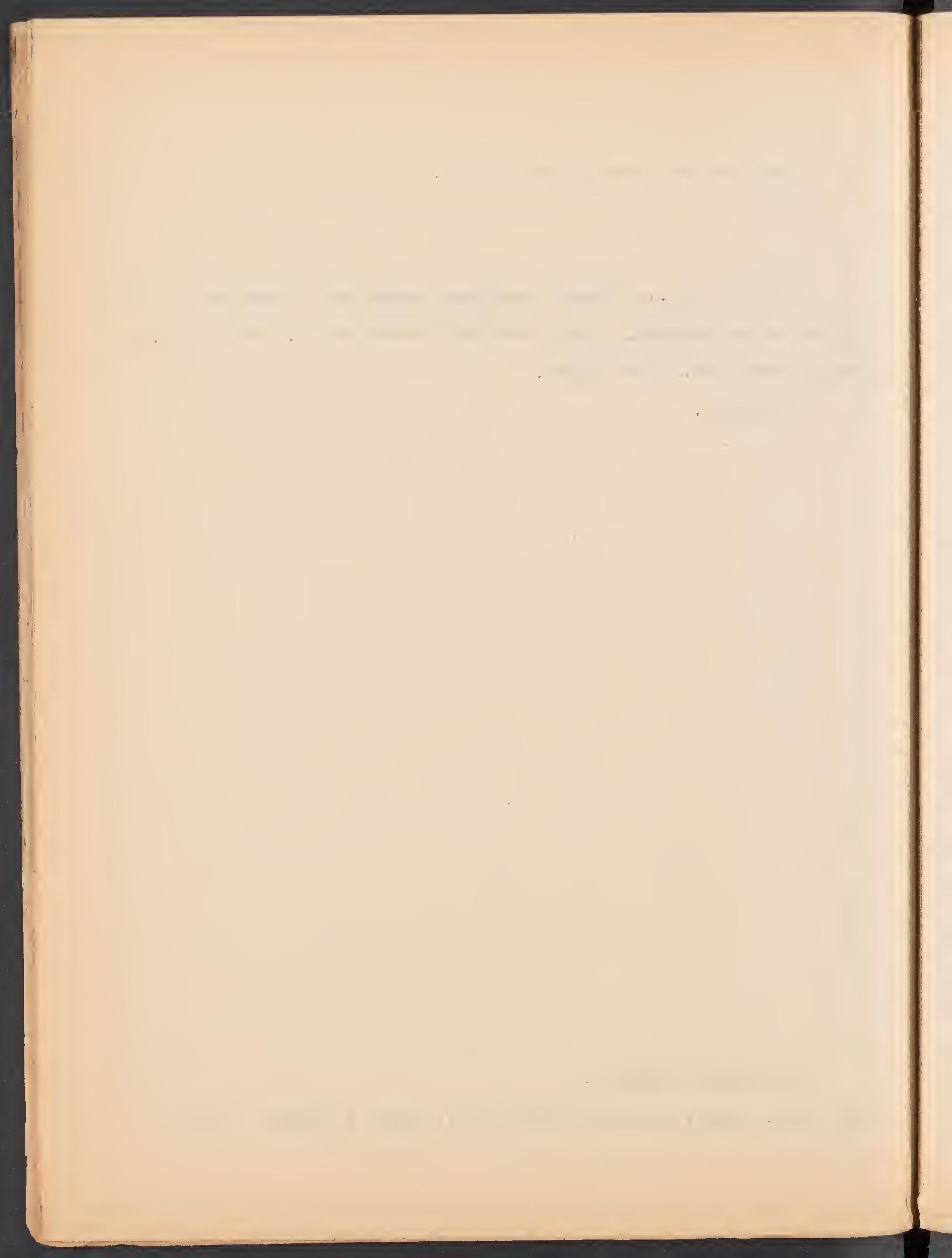


Favolus near reniformis.

Pileus eray, reticulate with darker lines;context thin. Dead loE.

Specimens examined:

Costa Rica, Iimon,tributary south, Rio Siquirres, Alt.160-180m., Catt, Dodee \& Thomas 5526 


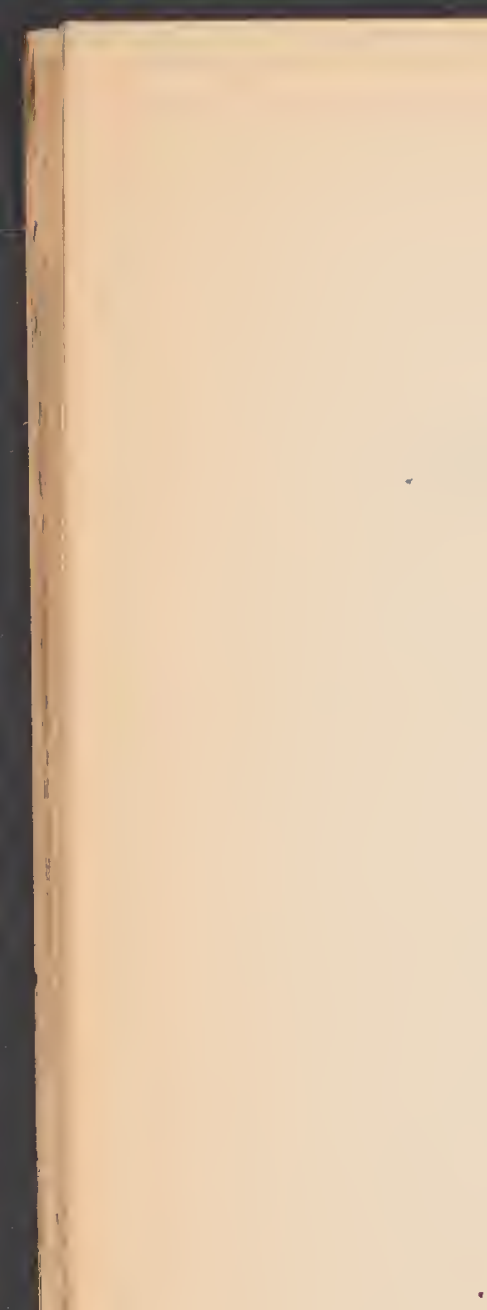


Favolus canerata group

Pileus $8 \mathrm{~cm}$.,cream-color,irregular, caespitose, margin slightIy tesselate, rest opauue, smooth surface; tomentose tibes decurrent on stipe, edges smooth; stipe short,white tomentose,below tubes, $2 \mathrm{~cm}$. \# $8 \mathrm{~mm}$.

\section{Specimens examined:}

Costa Rica, Limon, Barnstorf Farm, Ge.t, Dodee \& Thomas, 5524 



$$
282
$$

try Harasmius polyporoides.

collobo re sinus

New genus.

Pileus membranous ,2. cm., centrally stipitate, surface smooth, reticulate alone lines of sills or pores,light-pinkish-cinnamon;hymer. hymenium of interveined gills? concolor stipe,sjender, tough, $2 \mathrm{~cm}$. \# .7-1 mm. cinnamon or darker.

Dead leaf mould above R.Sicuirres.

Specimens examined:

Costa Rick, Limo, above Rio Siquirres, Alt.200-240 m., Catt, Dodge \&

Thomas

5522 

Flammula

Pileus $2.2 \mathrm{~cm}$.,ochraceous-buff to antimony-yelzow, expanded,fibrillose,scaly; gills close,broad,zinc-orange, adnexed; stipe slender, tapering downward, 15 \# 3-1.5 mm,fibrillose, scaly. Close to F.4642 from Coliblanco. Cespitose onfiecayinę 208 .

Specimens examined:

Costa Rica, Limon, Iivingston Farm, Alt.70-80m.,Catt, Dodee \& Thomas 5521 


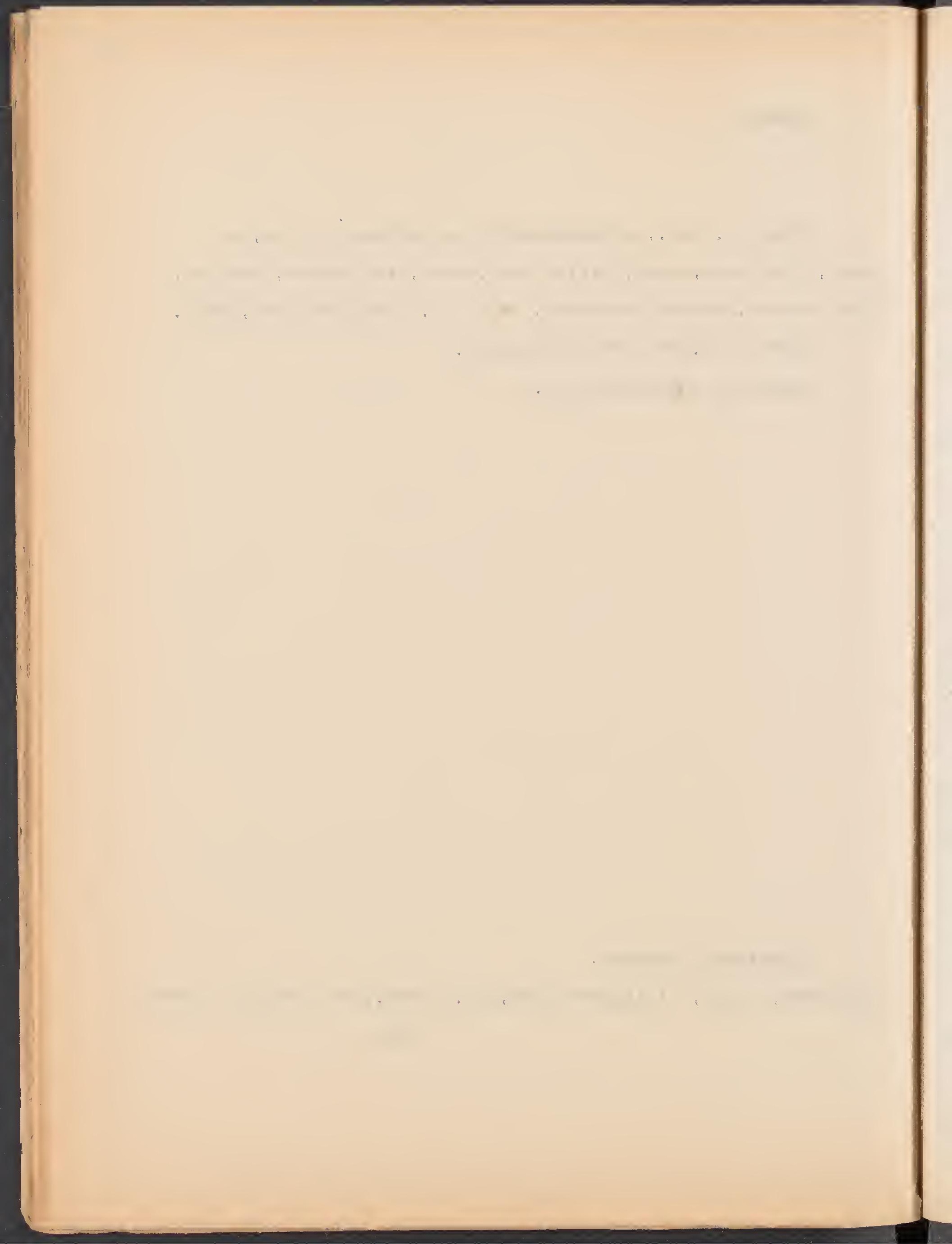



Flammula

Pileus about $1 \mathrm{~cm}$. diam; xanthine-ornage to zinc-orange with darker, pyramidal, fibrillose spines, depressed with broad umbo; gills broad, adnexed, close,xanthine-orange; stipe fleshy,broadening aboveffor context, slightly hollow below,fibrillose with faint remains of cortina, zinc-orange, lighter above, $1 \mathrm{~cm} \# 1 \mathrm{~mm}$.

Specimens examined:

Costa RIca, Limon, Iivingston Farm, Alt.70-90 m., Catt, Dodge \& Thomas 5520 


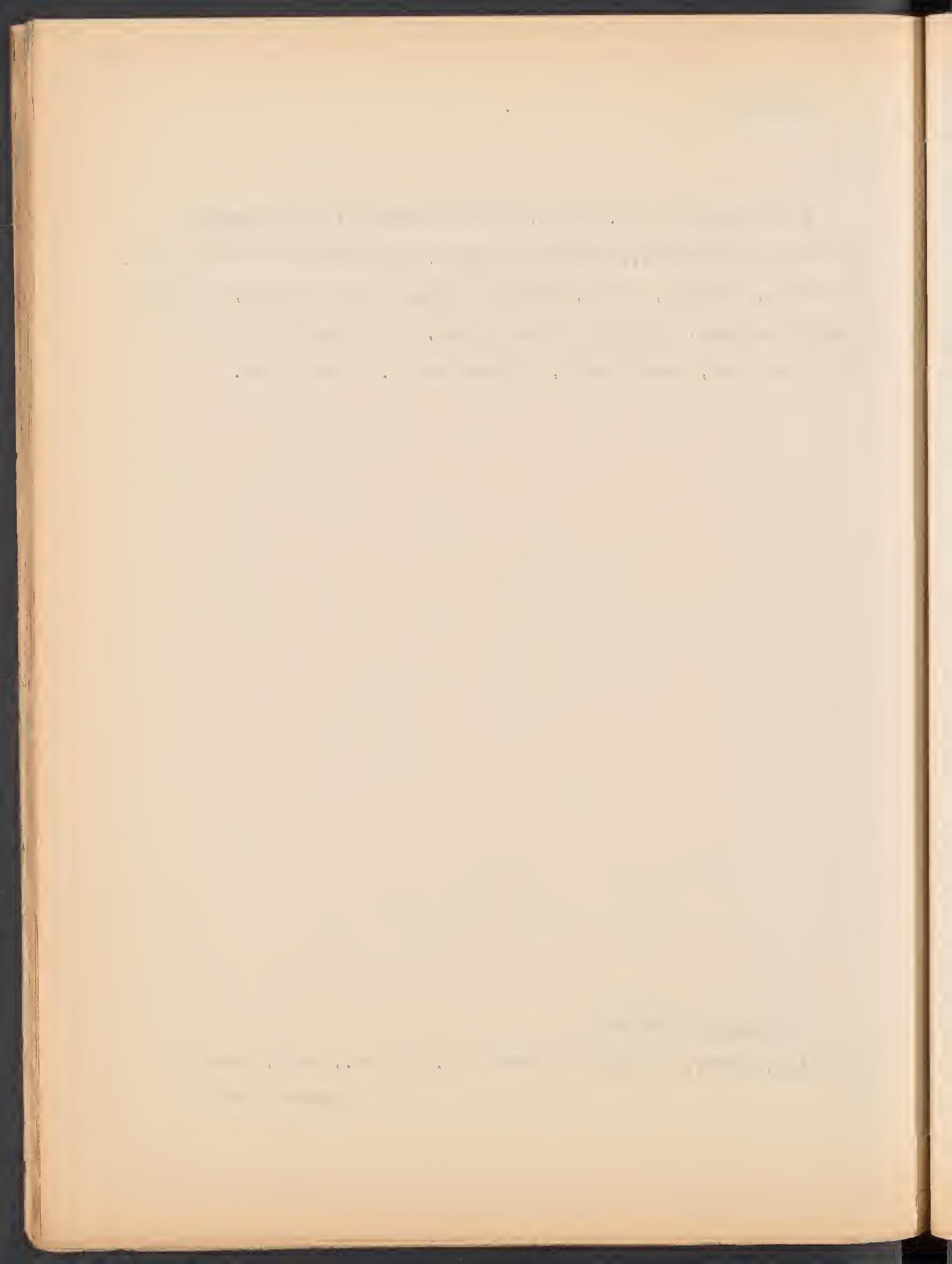


Flamrala subpenetrans in $\mathrm{Key}$.

On dead Logs.

Specimens examined:

Costa Rica, San Jose, Rancho Redondo, ALt.2150, Doage \& Thomas 5171 


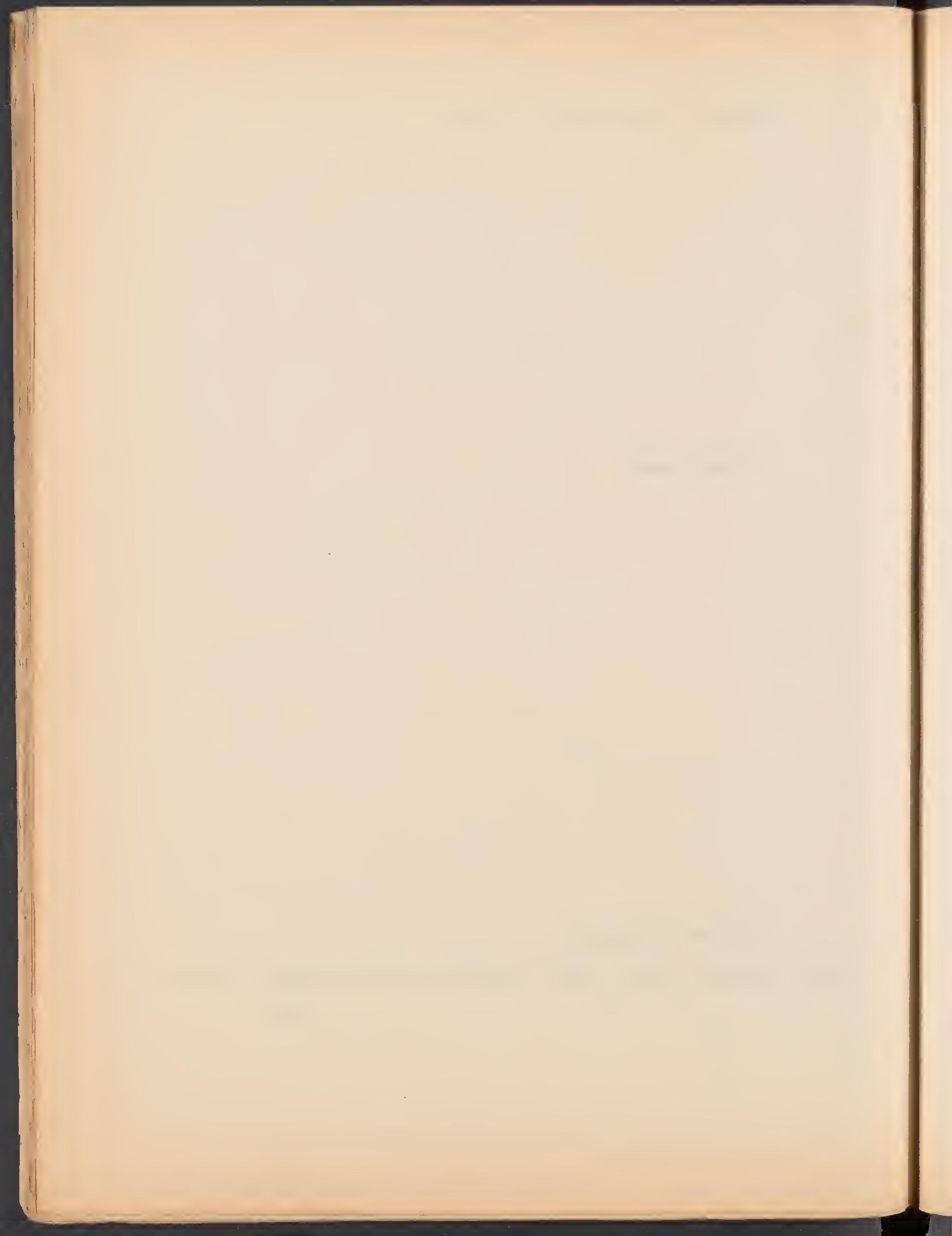


? Flammula

Pleus convex, margin scollopea, Iibriliose, scaly, Mars yellow to sudan brown; tLesh thin;gills narrow, crowaed, anate, cadmium-yellow, spotted with shestnut where bruised;stipe concolor with gills cart.,hollow, surface tibrous, tomentose.

Mossy 108 in potrero.

Specimens examinea:

Costa Rica, San Jóse, Rancho Redonao, It.2s00m., Doage \& Thomas 5170 
Flammula

Additional material; pileus surtace verrucose, punctate, sca 1 y

Specimens examined:

Costa Rica, San Jose, Rancho Reaondo, A Lt.2400m., Doage \& Thomas 5175 


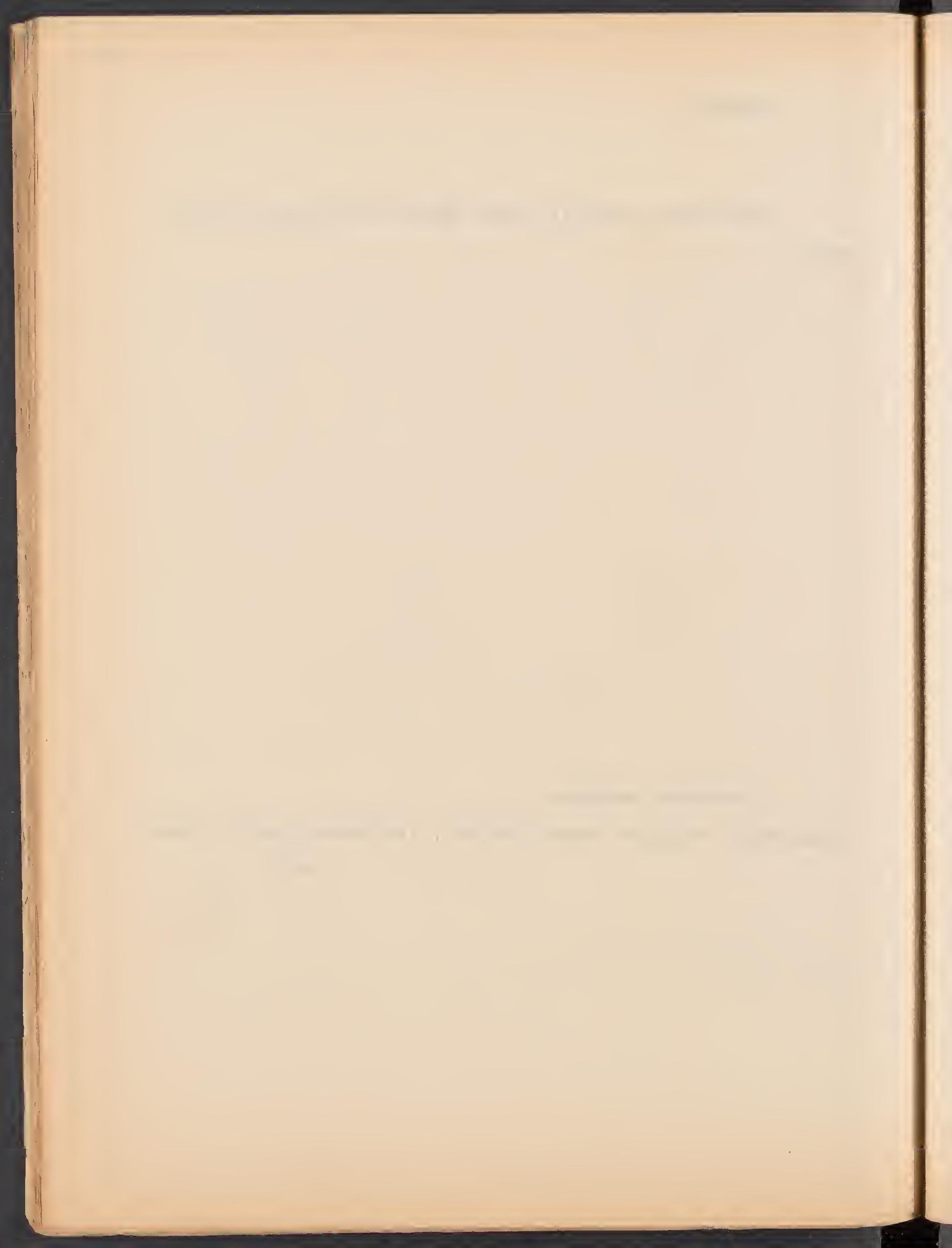


? Flammula

Plieus convex to expanded, up to $5 \mathrm{~cm}$., innate, Iibrillose, chestnut or somewhat lighter on margin, which is slightly appendiculate, not inrollea;gills adnate, broad, close, wax-yellow; Iesh thin, except on disc, watery(cart? $\phi$ of same texture \& continuous with stem;stipe I'leshy, cartilaginous, watersoaked, smooth, silky, concolor with gills abobe shading to color of pileus or darker below, $4 \mathrm{~cm}$ \#f $4 \mathrm{~mm}$.

hossy logs in potrero southe of house.

Specimens examined:

Costa Rica, San Jose, Rancho Redonao, Alt. 2160-2180, Dodge \& Thomas 5176 


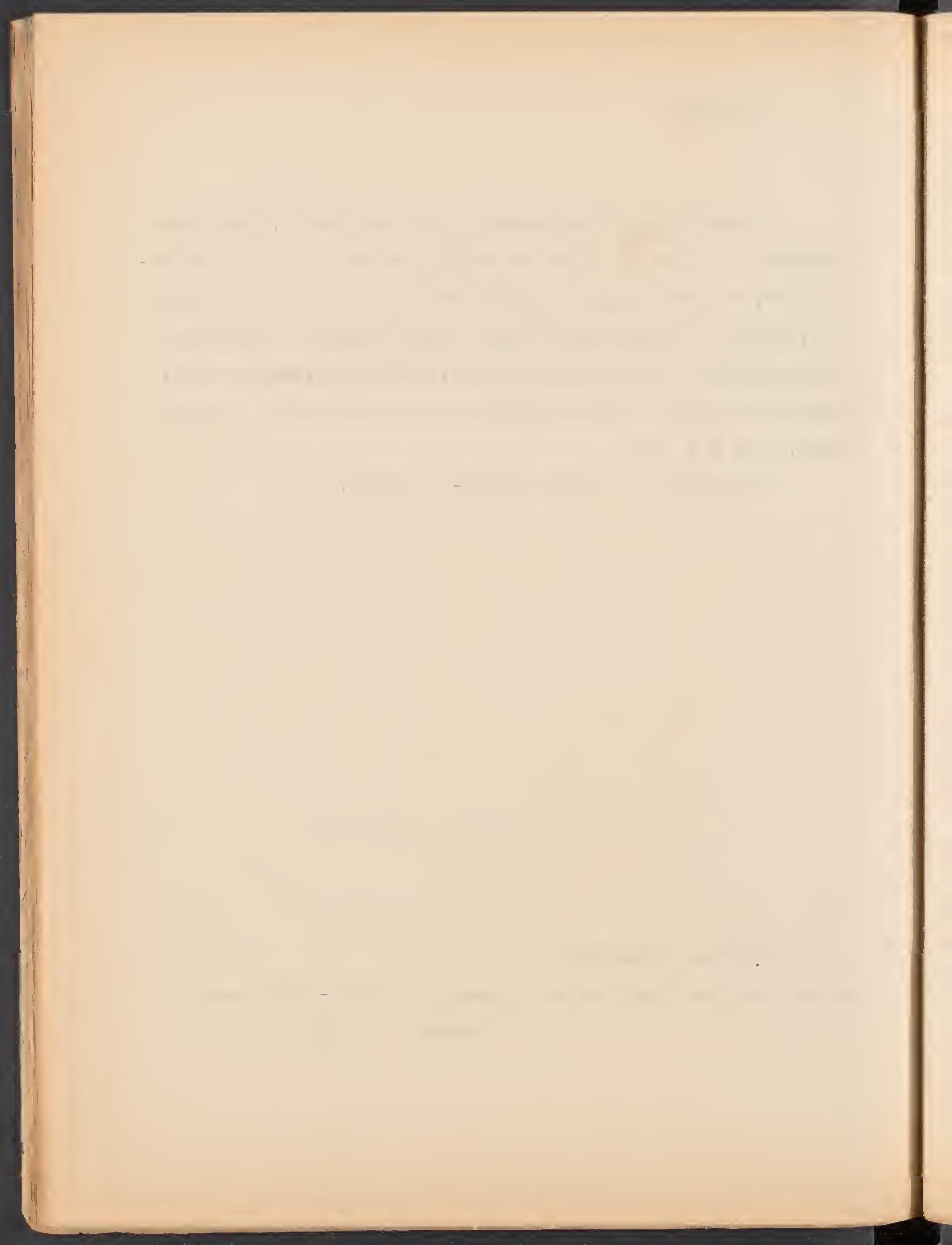


Flammula near chrysotrichus B \& C.

rlleus expandea, margin inrolled, tibrillose, maculate, mustara-yellow with ochraceous-tawny spots, up to $2 \mathrm{~cm}$. diamter; flesh medium mustard-yellow; g11ls close, crowaed, olive-brown, adpate; stipfe, slender, equa $3 \mathrm{~cm}$., mustard - yellow, somewhat darker in spots, very fibrillose.

on mossy trunk.

Specimens examinea:

Costa Rica, Cartago, Finca Coliblanco above Capelladas, Alt.I980m.

Smon: Marta, Dodqe 7233 Dodge \& Thomas 4642 


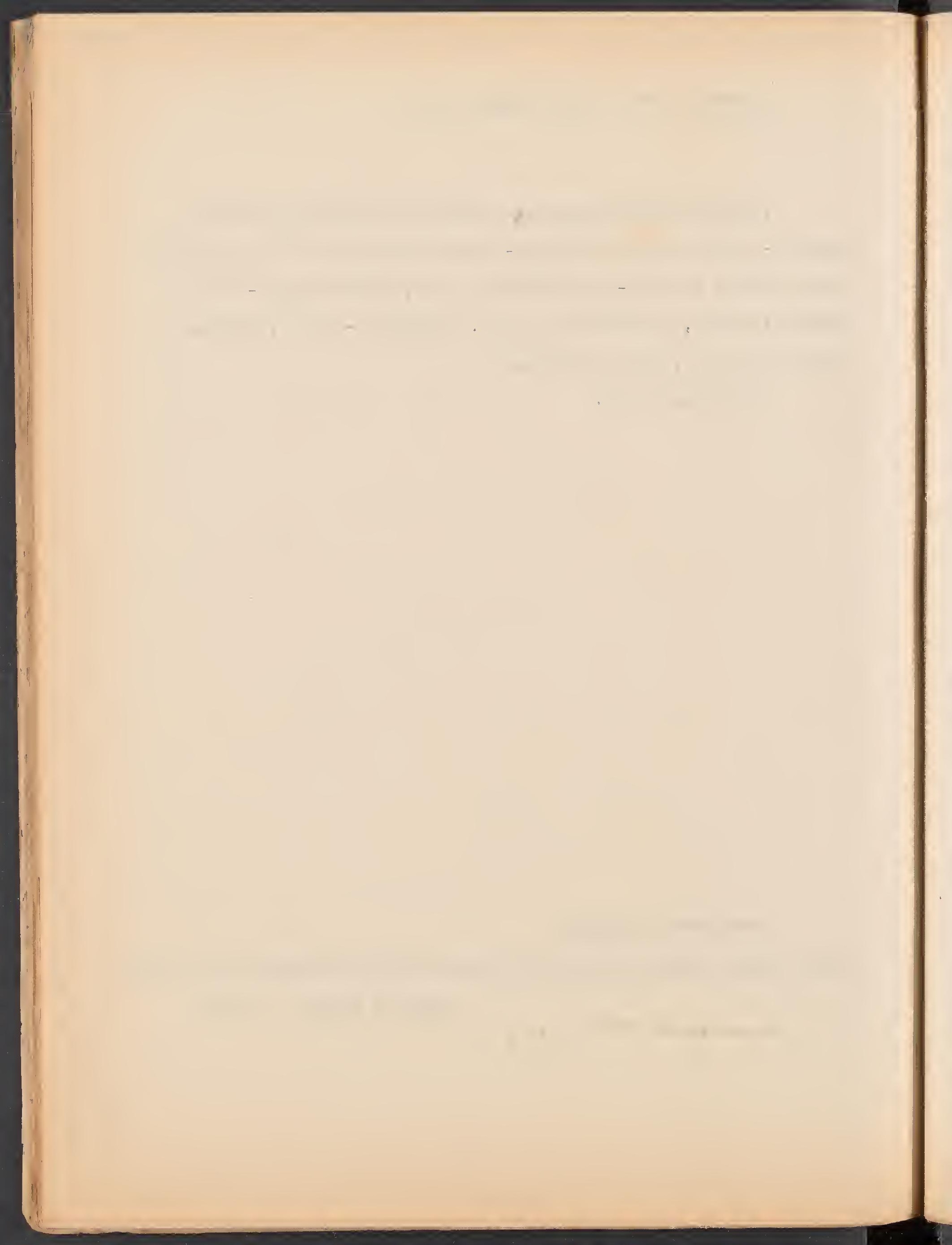


?Flammu La

Pileus flat or expanded, 11 brillose, scaly, amber-brown or 11ghter, $4 \mathrm{~cm}$; I'Lesh, fleshy, spongy, 11ght-yellow when f'irst cut, darkening on exposure, membranous on margin,gradually thicker to disc continuous with stipe, which may be hollowm, stuffea; gills antimony-yellow, zinc-orange, anate, margin og pileus not appendiculate; stipe more fibrousoraeshy $3.5 \mathrm{~cm}$ m $\mathrm{mm}$. coarsely fibrillose, striate, darkening on handing, conc.with gilis above $5 \mathrm{~mm}$. rest conc.pileus Mossy logs in potrero south of house.

Specimens examinea:

Costa Rica, Rancho Redondo, Alt. 2160-2180m., Dodge \& Thomas 
? Flammula

Specimens examined:

Costa Rica, Sen Jose, Rancho Redonao, Alt. '2150-2350m., Dodge \& Thomas $b 178$ 


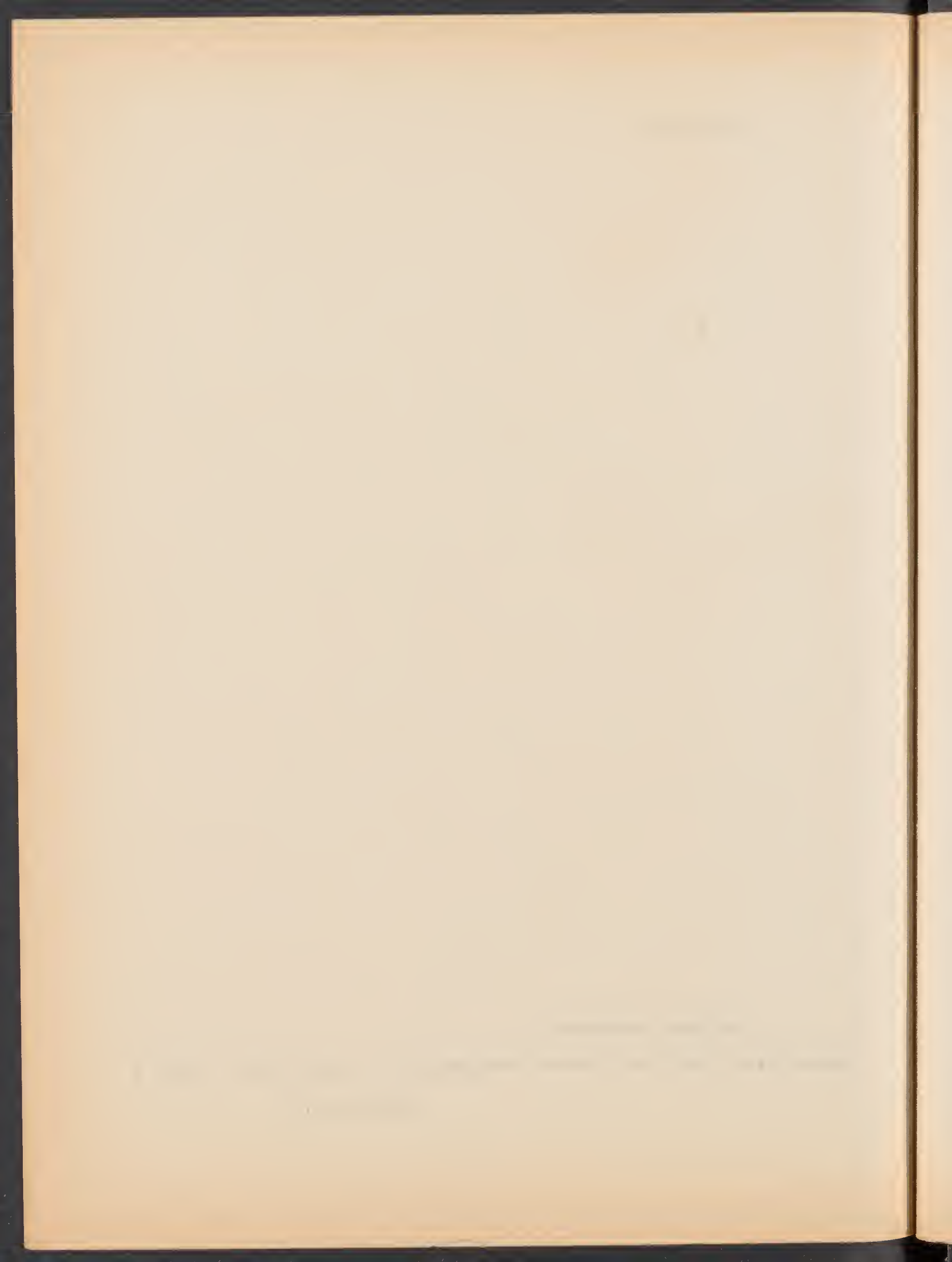


Hammula chysotrichoides in Key

Dead wood in potrero south of house

\section{Specimens examinea:}

Costa Rica, San Jose, Rancho Redondo, Alt.2160-2180, Dodge \& Thomas 5173 

Flammula

Similar to specimens ot Nov.6

Dead logs.

Specimens examined:

Costa Rica, San Jose, Rancho Redondo, Alt.2400m., Dodge \& Thomas 5180 


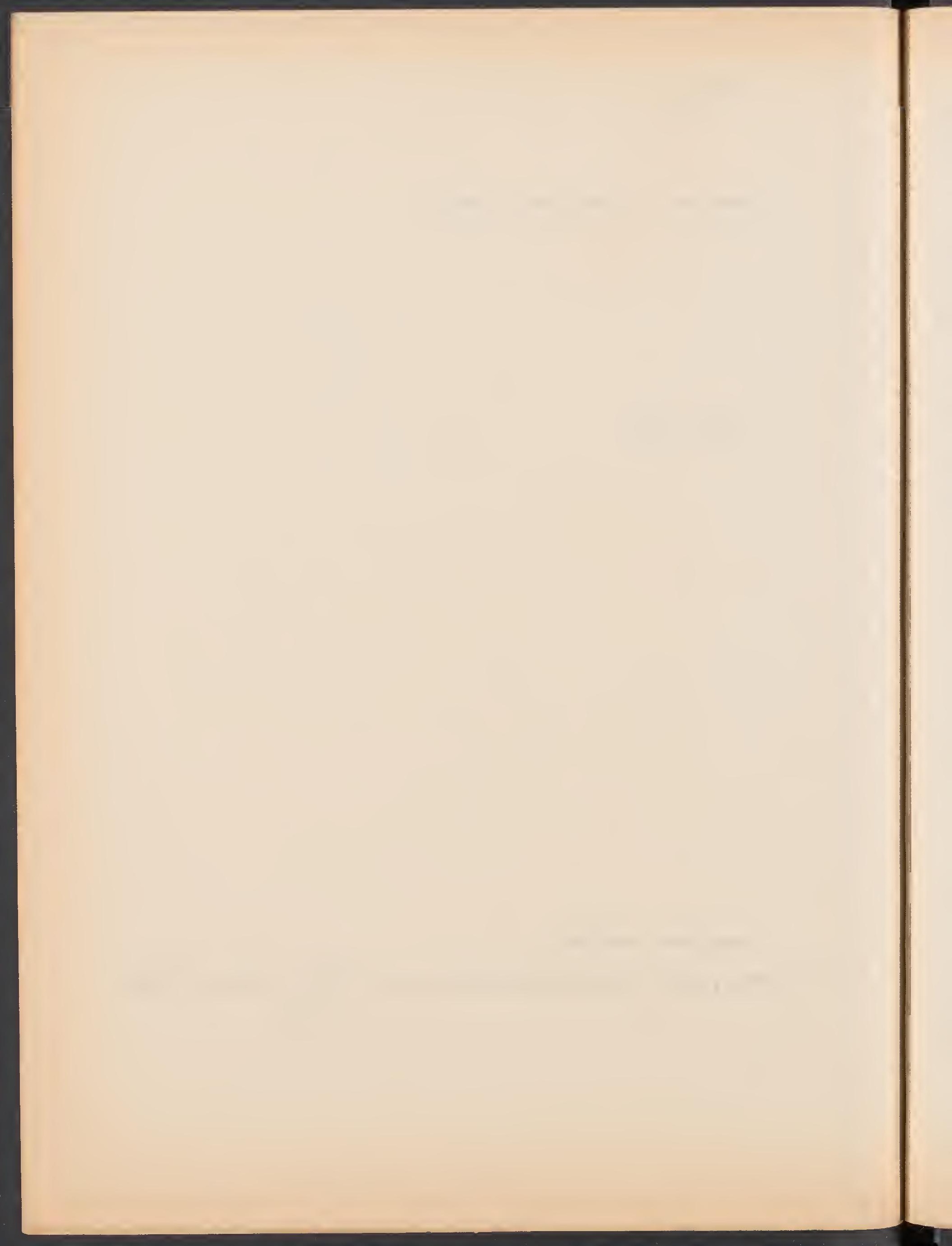


? Elammula

Pileus convex,margins inrollea, hablt ot_Lactarius, suriace innate, Ilibrillose, scaly, up to $8 \mathrm{~cm}$. diameter, variegated cinnamon-brown \& ochraceous-tawny, darker where bruised;flesh thick; gills close, anate, decurrent by line, yellow, Santrord's brown when bruisea;g1lıs narrow, primuline; stipe $25 \mathrm{~cm}$.\# $5 \mathrm{~mm}$. alamter, I1 brous, fleshy but slightly nollow, Ilbrillose without Sandiord brown on a primuline yellow, smooth ground: flesh darkening to ochraceoustawny on exposure to air. Mossy Logs in potero south of house. Rancho Reconoo

Specimens examinea:

Costa Rica, San Jose, Rancho Redoado, A Lt. 2160-2180m., Dodge \& 

? Flammula

P1leus irregularly expanded up to $5 \mathrm{~cm}$., surtace hygrophanous, scaly, auburn; gills close, subdistant, wide, sinuate, adnate,Mars yellow; stipe $2 \mathrm{~cm}$. \# $7 \mathrm{~mm}$. above, $5 \mathrm{~mm}$. below, concolor with gilis darker where handled.

Mossy logs in potrero.

Specimens examined:

Costa Rica, Sancfosegarancho Reaondo, Alt. 2250m., Doage \& Thomas 5179 


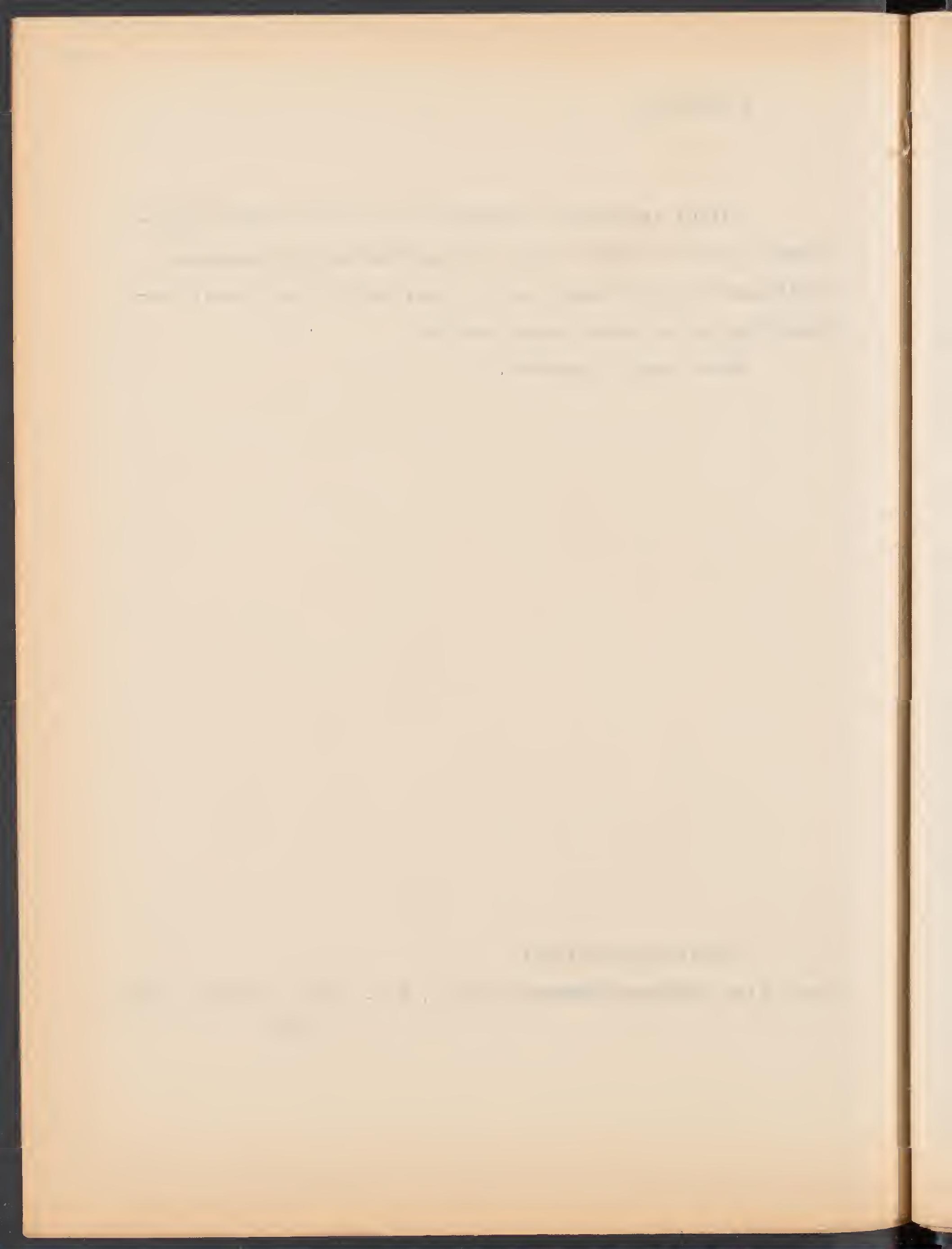


Flammula C

( See painting $\emptyset$

Color lighter;flesh only on disc;stipe long, slender;

pileus convex, pyramial tufts of hairs.

south potrero.

Specimens examined:

Costa Rica, San Jose, Rancho Redondo, Alt. 2150m., Dodge 501 s 

Flammula $B$

(Seepainting)

Pileus flat, subtomentose,fibrillose; flesh all the way to margin; stipe silky, fibrillose.

potrero south of house.

Specimens examined:

Costa Rica, San Jose, Rancho Redondo, Alt.2150 m, Dodge 5225 
Elammula

(ses painting)

Pileus up to $4 \mathrm{~cm}$. diam., convex, raw sienna, velvety, minute warts like Elaph. variegat. with shining dark apex; stipe cartilaginous not continuous with flesh, slender, surface tomentose, fibrillose darker than pileus, $4 \mathrm{~cm} \cdot \# \mathrm{~g} \mathrm{~mm}$. ; Illesh very thin; gills free, close, broad.

Logs in potrero south of house.

Specimens examined:

Costa Rica, San Jose, Rancho Reaodao, A1t.2160-2189, Dodge \& Thomas 5181 

Flammula

Pileus conica]. to applanate, broadly umbonate, innate fibrillo scaly up to $5.5 \mathrm{~cm}$ diam, russet disc shadine to antimony yellow bay maculate; fleh antimony yellow thick on disc, otherwise thin eills adnate, broad close antimony yellow; stpipe afleshy $3 \mathrm{~cm} x$ $7 \mathrm{~mm}$ taperingslightly downward, pruinose spotted with traces of eranescent annulus about $3 \pi \mathrm{mm}$ below gills, silky fibrillose white darkening to tough; gills ferruginous where wounded

Yossy lone in potrero $2400 \mathrm{~m}$

Specimens exarined

Costa Rica: San Jose, Rancho Redondo, $2400 \mathrm{~m}$ Jonx Dodge \& Thomas 5021 

Flamroula A

(See painting)

Pileus color darker, flesh only on disc;stipe slender \& short, hemispheric to convex, tomentose, fibriliose.

South potrero.

Specimens examined:

Costa Rica, San Jose, Rancho Redondo, Alt. 2150 m., Bodge 5012 

Elammula

Plleus scaly (See previous collection)

Genera 1 locality

Rotten $10 g$

specimens examined:

Costa Rica, San Jose, Rancho Redondo, Alt. 2400m, Dodge \& Dode Thomas 5226 

Flammula

(See painting)

Specimens examinea:

Costa Rica,ALjeela, San Isiaro,Alt. I300m.,Doage \& Valerio

4756 

?HLamula

Plieus expanded, slightly umbonate, up to $4.5 \mathrm{~cm}$, surface glabrous, innate, f'ibrillose, amber-brown to antique-brown, becoming Mars yellow to raw sienna towara margins;ilesh antimony-yellow, fleshy, subcartiliginous next gills, continuous with stipe; gills sinuate,adnexed to almost iree,broad, close, tawny; stipe short \& stout, $18 \mathrm{~mm}$. \& $6 \mathrm{~mm}$. tomentose,antimonyyellow, becoming tawny where bruised, fleshy subcartilaginous, hollow, stufted.

Mossy logs in potrero south of house.

Specimens examinea:

Costa Rica, San Jose, Rancho Reaonao, Alt.2160-2180m.Dodge \& Thomas 5172 . 


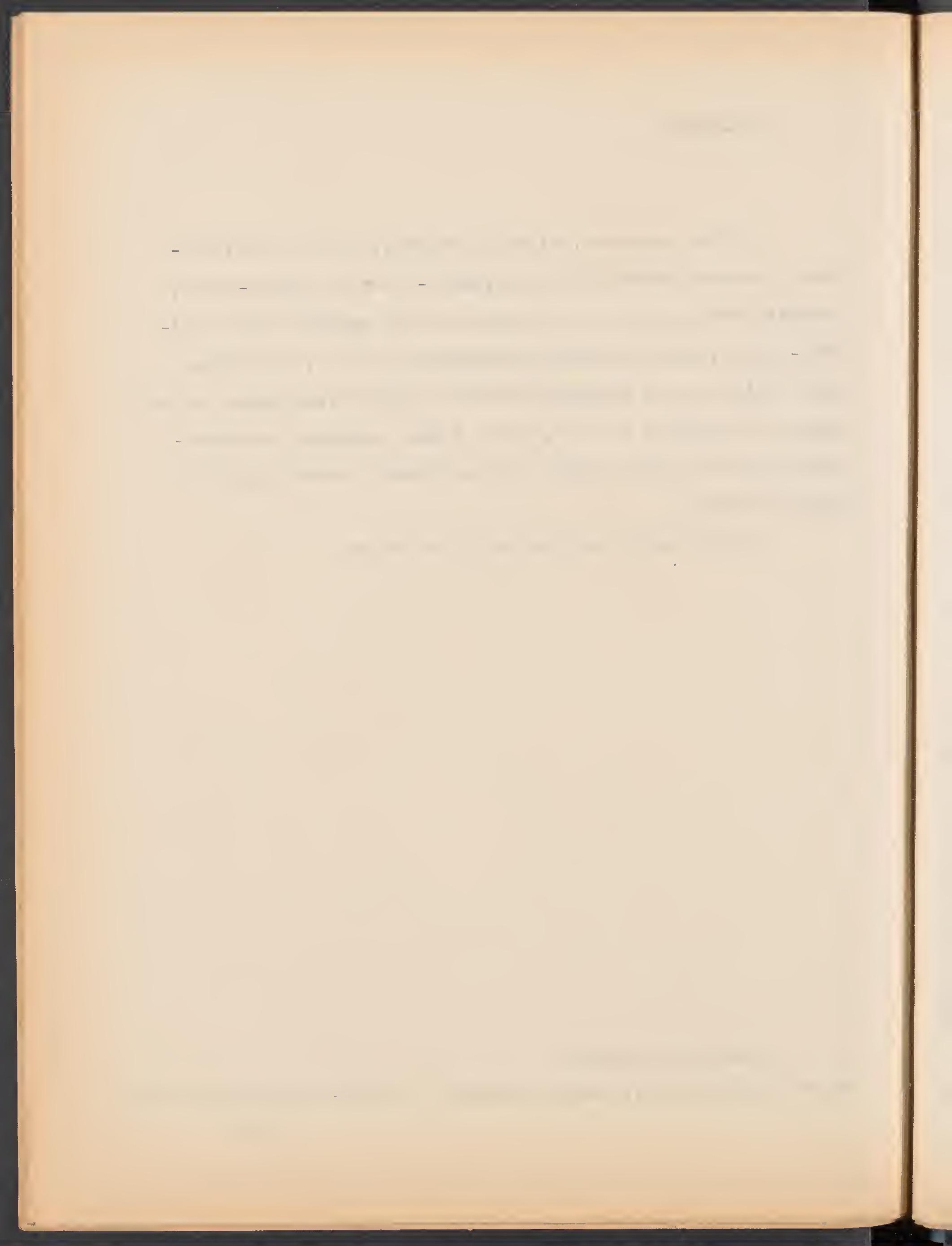


3F Lampula penetrans in icey

Pileus expanded, convex $3.3 \mathrm{~cm}$, surtace wrinkled, scaly, fibrillose, tawny to Mars brown; gills sinuate, ventricose, close, yellow-ochre to ochraceous-tawny; stipe $3 \mathrm{~cm}$. \# b mm. not taperi ing surface, fleshy striate, f'ibrous, concolor above, darker below. Mossy logs.

Specimens examined:

Costa Rica, San Jose, Rancho Redondo, Alt. 2250m., Dodge \& Thomas 5174 

Flammula

Pilei cespitose conjc to nearly plane, $1.6 \mathrm{~cm}$ dim, flesh thin except on djsc, innately fibrillose, disc yel.ow ochre shadine to mustard yellow margin inroJ.jed; elils broad, close, adnete to githo-

Iy decurrent, brown; stipe smooth concoloroua, sljuhtly sulcete in youne specimens, $2 \mathrm{~cm} \times 2 \mathrm{~mm}$

Decaying wood

Costa Rica:Limon, Marta $20 \mathrm{~m}$, Dodee 7233 

Flammula

Pileus up to $4 \mathrm{~cm}$, sparsely aculeate scaley, antimony pyellow, marerin thin, smooth; gills sinuate adnate, close, ventricose, zinc orance; stipe striate fibriljose, equal or taperine slightly downward $4.5 \times 4-3 \mathrm{~mm}$, zinc orance or lichter

Decaying $\&$ frond of swamp paIm(7308) charred decaping logmtanamal

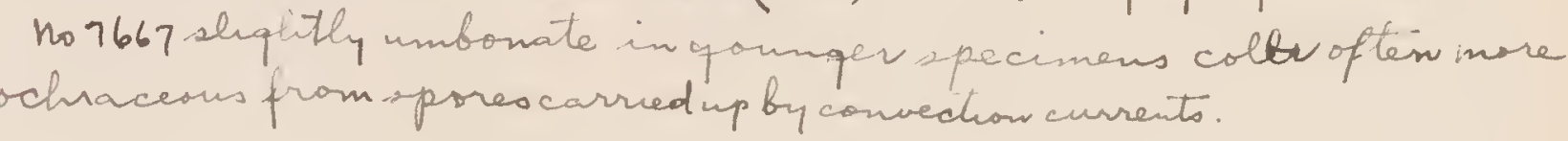

Costa Rica: Limon, Castilla $20 \mathrm{~m}$, Dodge 7308. Puntaremas, Prentogumeng $10 \mathrm{~m}$ Sodge 7667 



\section{Ga]era}

Pileus campanulate, hyerophanous, Brussels brown, with antimony yellow djsc $6 \mathrm{~mm}$; gill.s adnate; stipe cartilaginous slender $2 \mathrm{~cm} \times 1 \mathrm{~mm}$, brussels brown or darker.

sPecimens examined:

Costa Rica: San Jose, Rancho Redondo $2200 \mathrm{~m}$, Dodge \& Thomas 51.07 


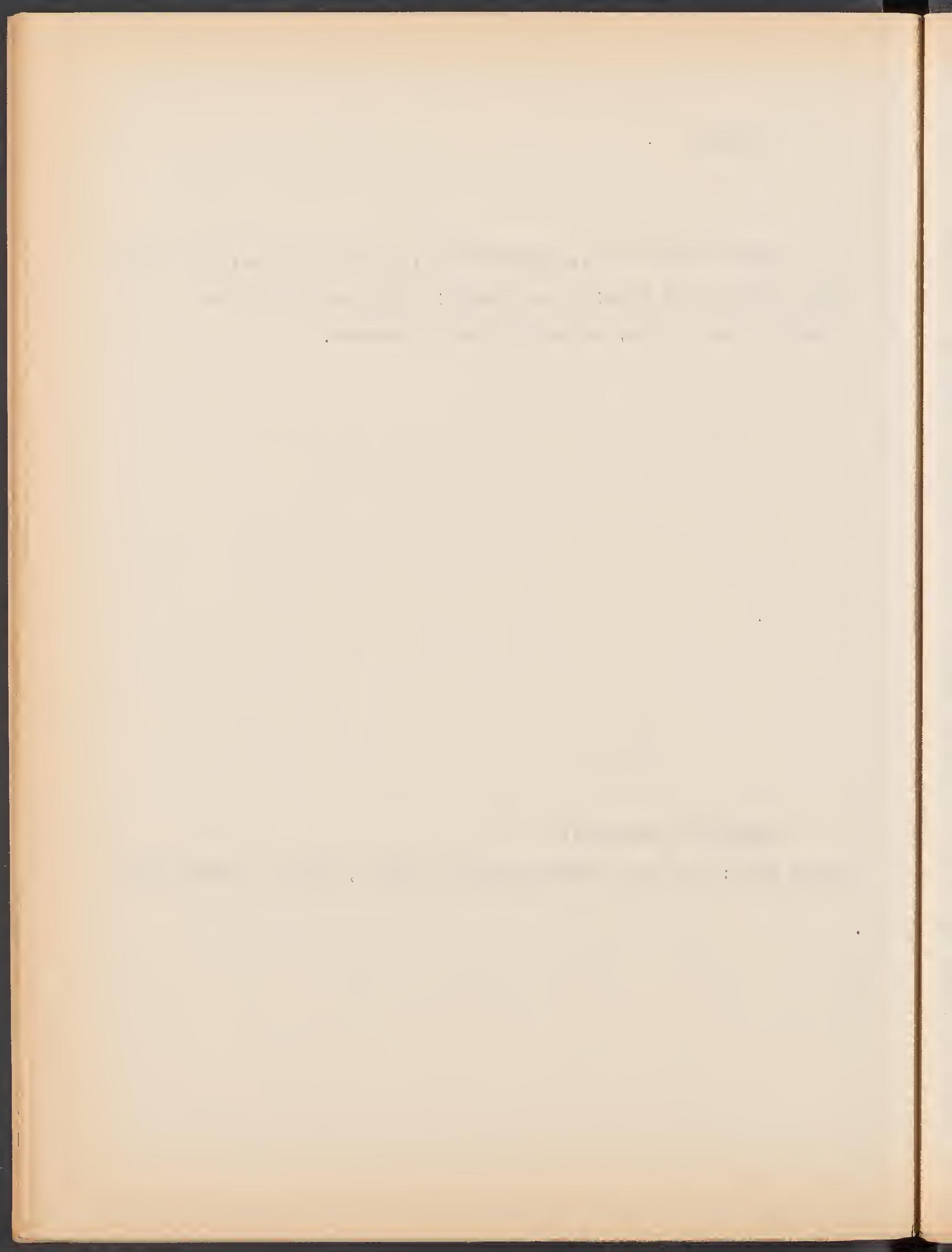


Galera

Pileus campanulate,margin striate, surface minutely tomertoee, $7 \mathrm{~mm}$. diameter, antimony-yellow, margin russet; gilis broad, distant, adnate, concolorous; stipe slender, $20 \# 1 \mathrm{~mm}$. concolor cartilaginous. Mossy bank.

Specimens examined:

Costa Ruca, San Jose, Rancho Redondo,Alt. $2100 \mathrm{~m}$, Dodge \& Thomas 5350 

Galera

Piles expanded, $7 \mathrm{~mm}$ margin shortly dentate lacerate, velvety tomentose, cinnamon buff on disc, margin lighter; gills close, rentricose, sinuate adnate, concolor, stipe silky concolor $20 \mathrm{x}$ $0.7 \mathrm{~mm}$

Mossy bank on roadsiale

Specimens examined:

$2100 \mathrm{~m}$

Costa Rica: San Jose, Rancho Redondo Dodee \& Thomas 5377

quayabillos, $2300 \mathrm{~m}$ Nodgetgoerger fuly?. 

315

Galere near Hypni

Pileus campanulate to convex when expanded $2.2 \mathrm{~cm}$ diam, velvety. deeply striate to disc, cracking radially over gills as in Hiatula, cinnamon disc shading to ochraceous orange, Inargins flesh, litter alone cracks, mare in thin as in Coprinus but not enrolled; $\varepsilon j$ I Is brown, medium distance and width, adnate; stile slender, silky, nearly white above to ochraceous orange below and subavellaneous in $\mathrm{X}$

old specimens, cartilaginous $3 \mathrm{~cm} 3 \mathrm{~mm}$

Decaying log in jungle

Costa Rica:Iimon, hills opposite Hamburg $40 \mathrm{~m}$, Dodge 7314 

Galera

Psathyra

Pileus conic, campanulate, not umbonate, $12 \mathrm{~mm}$. diameter, $9 \mathrm{~mm}$. high, substriate, mummy-brown with lone slnder,pyramidal spines ober whole surface, margin appendiculate; Eills crowded, quite thick, edces adnexed; slender cartilaginous, white with dark-brown fibrils, $2 \mathrm{~cm} \# 1 \mathrm{~mm}$. often stellately arranged ( universal veil) above, white, pruinose where gills join stipe.

Decaying wood.

Specimens examined:

Costa Rica, Limon, Rose Hill above Rio Pacuare, Alt, 100m.,Catt, Dodge \& Thomas 5516 

Gatera ?

P1leus convex,hygrophanous, $7 \mathrm{~mm}$.,pruinose, margin very faintly striate, clay color, disc cinnamon-buif; gills broad, adnexed, cinnamon buff, close; stipe cinnamon-buff, silky, cartilaginous, 15 \# $1 \mathrm{~mm}$.

Mossy rank.

Spec1menseexamined:

Costa Rica, San Jose, Rancho Redondo, Alt.2140 m., Dodge \& Thomas 5349 



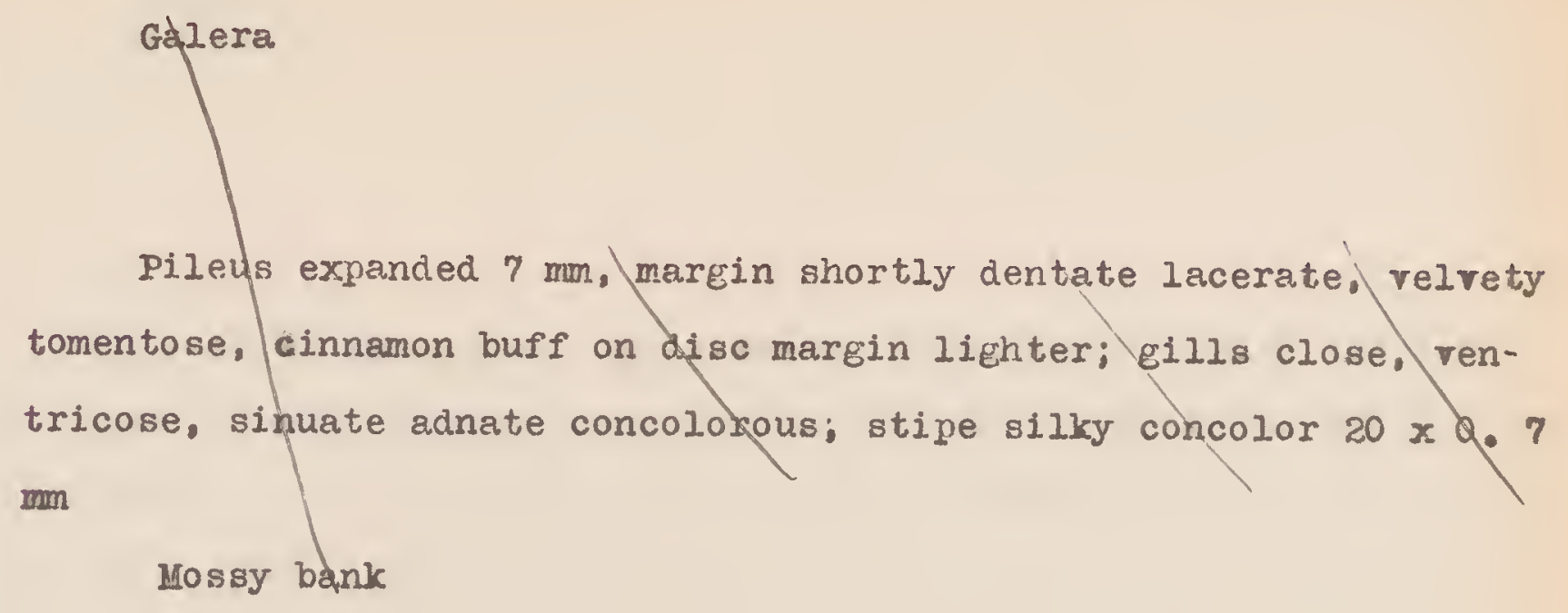

Specimens examined:

Costa Rica: San Jose, Rancho Redondo, $2100 \mathrm{~m}$, Dodge \& Thomas 5377 

Hebe

Sxocisrioma

Pileus expanded, subumbonate, $8-10 \mathrm{~cm}$ diameter, viscid, minutely fibrillose undergel, mummy brown disc to Dresden brown whith white shining margins; eills broad very close, sinuate adnate, chamois to isabella color; stipe fleshy fibrous 6-8 cm $\times 12-10 \mathrm{~mm}$, fibrillose silky, brown reticulate with remains of viscid gel, subbulbous below

In earth bank

Specimens examined

Costa Rica: SanJose, Rancho Redondo, $2450 \mathrm{~m}$, Dodge, Dodge \& Thomas, 5405 


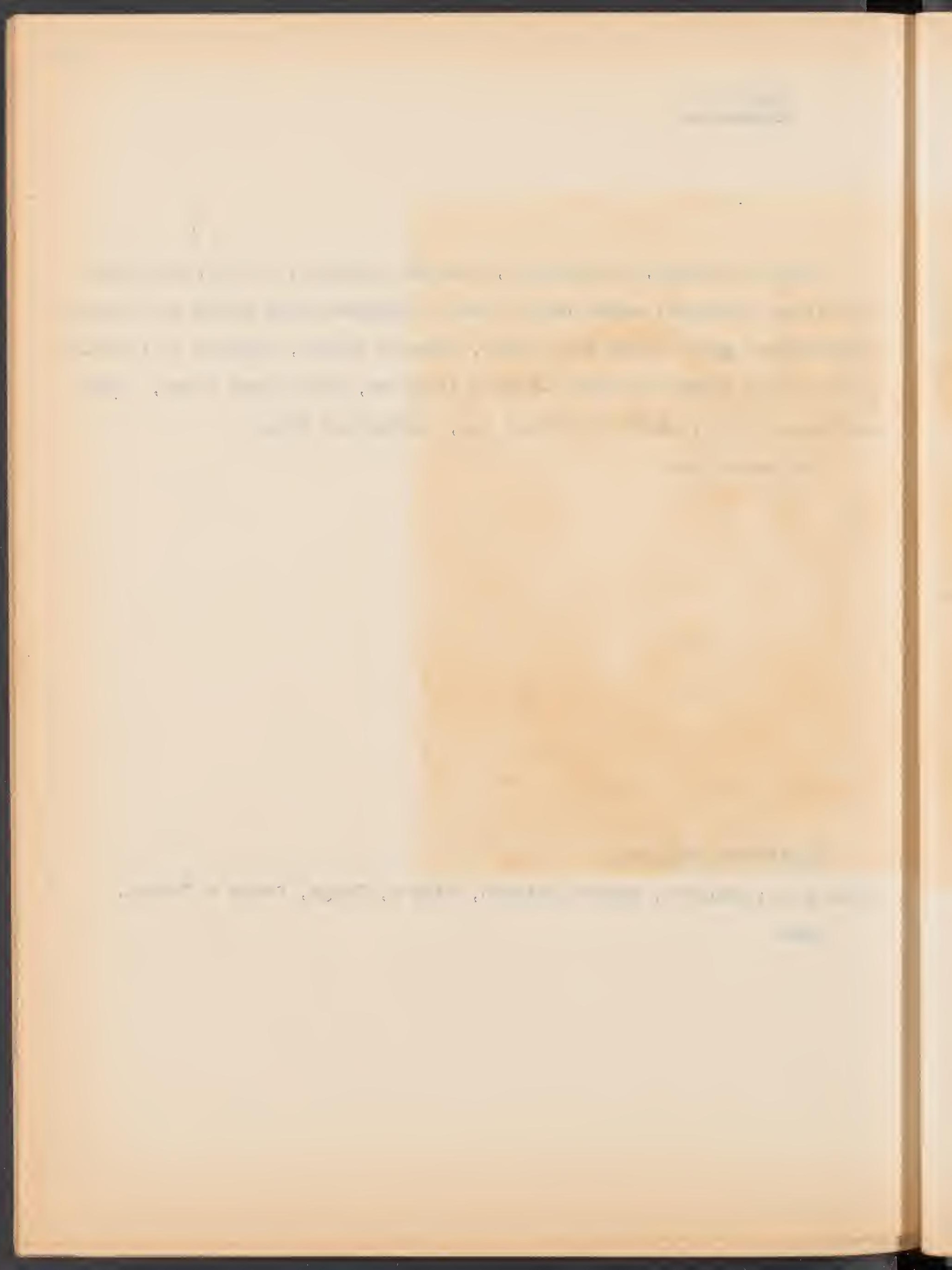


Hebeloma near subincarnatum

Pilei cespitose, expanded, alichtly umbonate, moist with occasion al citritne yellow remains of universal veil, disc isabella shading to colonial buff, margin sliehtly pappendiculate, amooth; gills sinuate, with slight decurrent tooth appearine nearly free, broad, close, isabella color, thin; stipe colonial buff with isabeline fibrils, slender, swollen below, $3.5 \mathrm{~cm} \times 3-4 \mathrm{~mm}$

In mould on very rottenlog in deep jungle

Specimens examined:

Costa Rica: $\mathbf{S}$ Heredia, Cerro Central de ZurG $u$ i $1620 \mathrm{~m}$, DodEe, Valer io \& Thomas 6128 
Hiatule or n.E.

Pileus lare, membranous lamellae splittine down back ochraceous alone lines betweengiJjs and on disc where lines meet; fills transparent; stem citrine, fragile very lone, slender; ring broad, pendent veryfratile

Decryine lewres in Ture

CostaRica: Iimon, Los Negritos $20 \mathrm{~m}$, Dodee \& Nevermann 7241 
Iiacules

Benconii c: oup

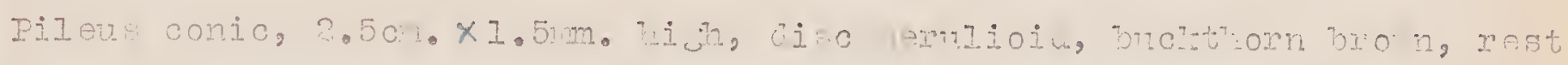

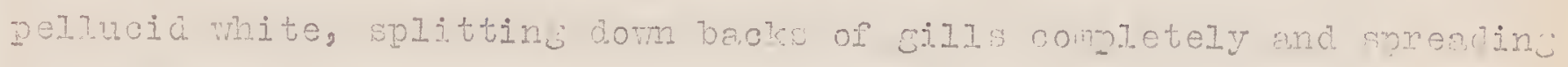

Gills white, subciatant, free fron stipe, broco

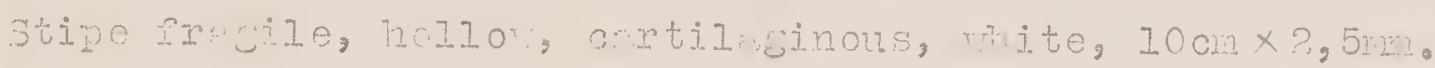

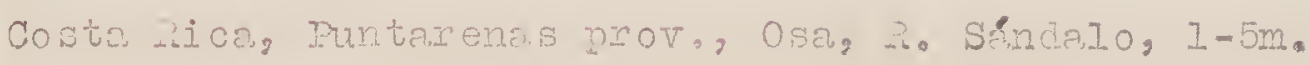



Hygrophorus near aurantia

Plleus conical, umbonate, faded; gillis free, ventricose, distant, apricot-yellow; stipe fragile, silky, apricot-yellow

Specimens examined:

Costa Rica, San Jose, Rancho Redondo, Alt.2200-2600m. Dods \& Thomas 5237 


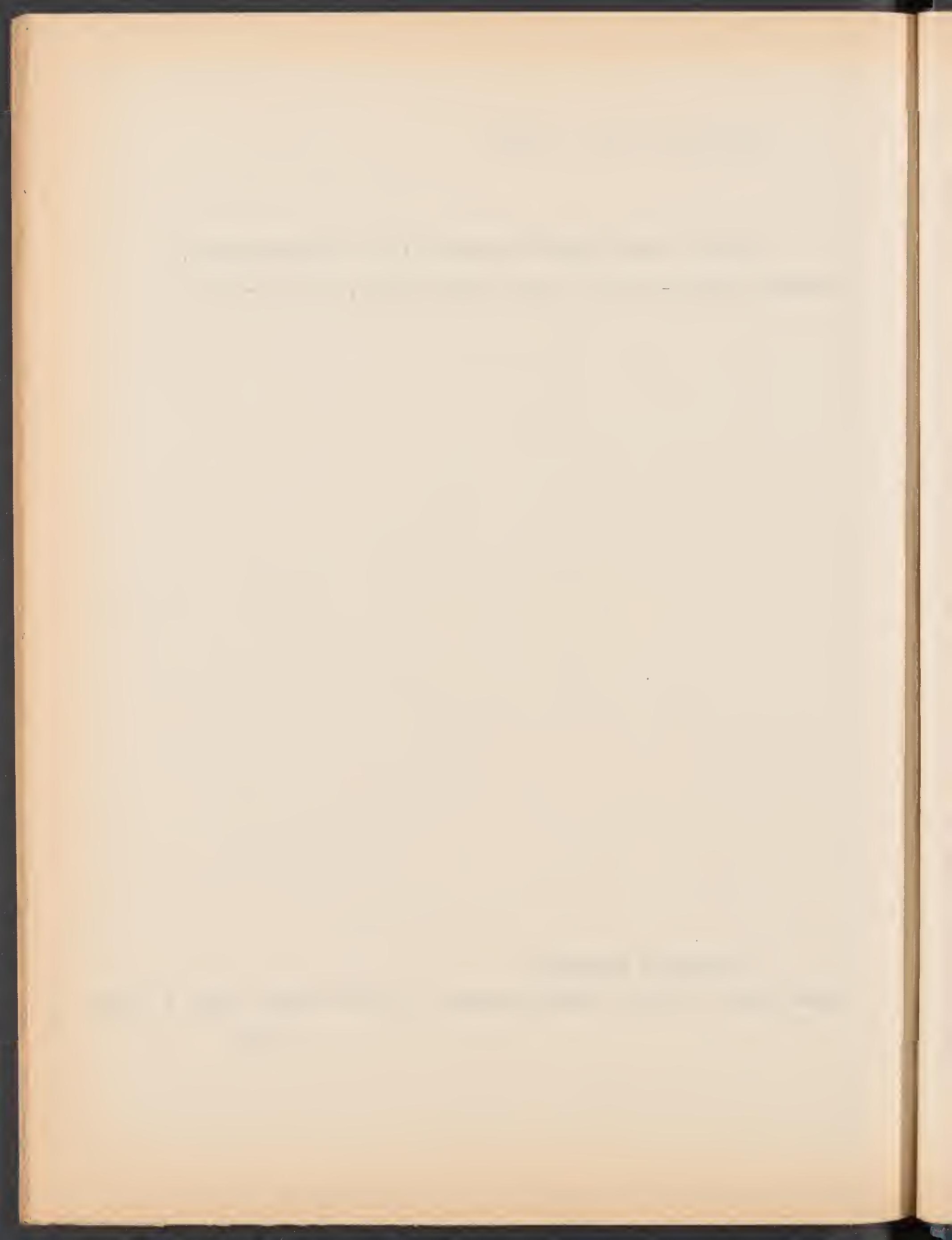


Hygrophorus

P1leus campanulate, $8 \mathrm{~mm}$.,Brazil red, becoming orangechrome on disc, viscid; flesh apricot-yellow; gills adnexed, apricot-grange, distant, thick; stipe fragile,hollow, inflated, belvo cartilaginous, pale lemon-yellow below, to oranse above, $1.5 \mathrm{~cm}$. \# $4 \mathrm{~mm}$.

Mosay 108 in potrero south of house.

Specinens examined:

Costa Rica, San Jose, Rancho Redondo, Alt.2180-2220m. Dodge 5139 


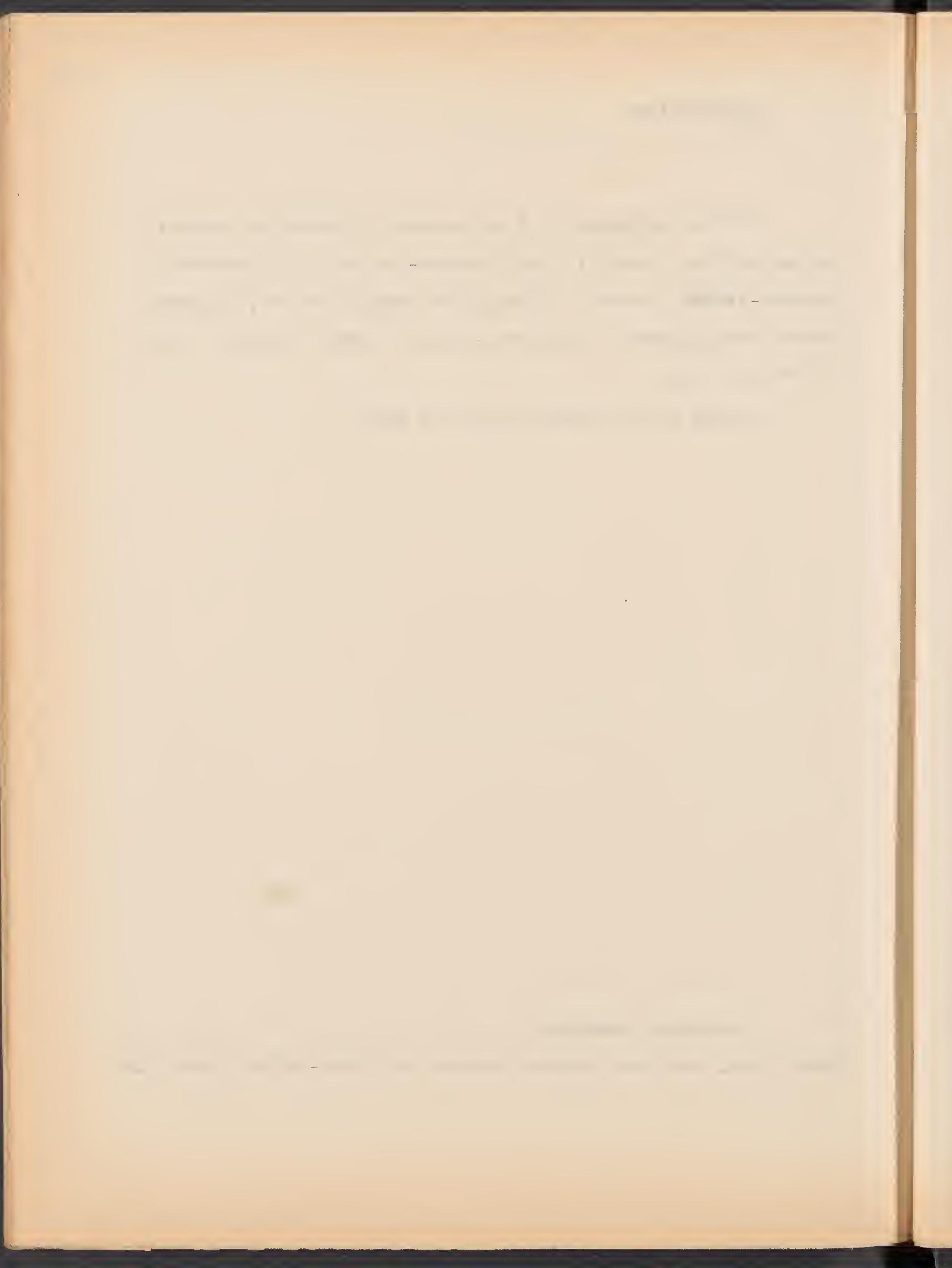


Hygrophorus near subminiata

Plleus bright red, convex to plane or slightly depressed; gills distant, deep red with white edges;stipe red above, bright yellowa colors fading on arying.

Among mosses.

\section{Specimens examined:}

Finca Coliblanco

Costa Rica, Cartago,a above Santa Theresa de Capelladas,Alt.1980 
Hygrophorus conicus group

(See painting)

Pileus campanulate with sharp, pointed umbo, blackening on drying, not viscid, moist, innate, fibrillose, margins inrolled; flesh very thin; gills free, narrow, orange-buff,blackening; stipe fibrous, cartilaginous, hollow, silky, fibrous without. Plieus attached to underside of huge, moss-covered log!

Specimens examined:

Costa Rica, Cartago, Finca Coliblanco above Sta.Theresa de Capelladas, Alt. about $1800 \mathrm{~m}$., Dodge \& Thomas 

Hy\&rophorus near Farlei

pileus convex, deeply umbilicate, $3.5 \mathrm{~cm}$, Mars waw orange over gills, Mars yellow hetween, margin thin, somewhat lacerate; gills adnate with decurrent tooth, broad, distant, dadmium jellow; stipe tapering downward $5.5 \mathrm{~cm} \times 4-3 \mathrm{~mm}$ lemon chrome

Costa Rica: puntarenas, Osa, between R. Nuevo and Pto. Jimenez $10 \mathrm{~m}$ Dodge 7651 

Hy grophorus

Pileus much depressed, attacired by myxomycetes, which may account for lack of top of plieus, now deeply infundibuliform, cartilaginoug between strontian yellow \& yellowish-citrine, $6 \mathrm{~cm}$. diamter, slightlydecurrent, thick, waxy, concolorous; stipe hollow $4 \mathrm{~cm}$. \# $8 \mathrm{~mm}$. alameter, cart1laginous, concolor.

Under Bambusoia grasses in soll. 

Hyerophorus

Pileus $3.5 \mathrm{~cm}$, flat, umbilicate, greenish black, hygrophanous; gills subdistant, ventricose orange yellow, adnate;stipe chestnut hollow, cartilaginous, $25 \times 4 \mathrm{~mm}$, end proken off

Specimens examined

Costa Rica: San Jose, Rancho Redondo $2100 \mathrm{~m}$, Dodee, Dodee, \&Thomas 5366 

Hygrophorus (Hygrocybe)

Dileus $3.5 \mathrm{~cm}$, Begonia rose, minutely fibrillose, margin irregularly lacerate, flesh thinigills proad, subdistant, hermosa pink, free or nearly so; stipe fracile, subcartilarinous, white above, coral pink shadine to flesh ochre below, $5 \mathrm{~cm} \times 5 \mathrm{~mm}$ with fusiform base $7 \mathrm{~mm}$ in diam.

Leaf mould at foot of tree inthe edge of the jungle

Gpecimens examined:

CostaRica: Heredia, Cerro Central de Zurqui, $1620 \mathrm{~m}$, Dodge, Valerio \&. Thomas 6022 
$\mathrm{Co}$ 
$33 i$

Hygrophorus bella?

subcaespitosa?

flammea?

Pileus Brazil red fading; stipe light cadmium. (5564)

Pileus expanded, scarlet to scarlet red, margin sightly more yellowish $11 \mathrm{~m}$ in diameter margin sightly striate; gulls de slant medium adnate to subdecurrant pale onangeyellow tow ht' stipe scarlet to orange $2 \mathrm{~cm} \times 2 \mathrm{~mm}$ fragile $(7713,7714)$

Pileus conic expanded very moist (probably viscid) desc rufous margin between lighteadminm and lemon chrome; gills the whit veaslyfree stipe $3 \mathrm{~cm} \times 5 \mathrm{~mm}$ white fleshy surface fbrilloze (7679)

Specimens examined:

Costa Rick, Iimon, above Rio Siquirres, Alt.220m., Catt, Dodge \&

Thomas 5564

Puntaremas ora near Esters Sandals 5-20m Dodge 7713,7714.?7679 

?Hygrophorug variologus Fr.?

(See also painting)

Specimens examined:

Costa Rica, San Jose, San Jose, parque Bolivar, Alt.1179 m. Doage

4318 

Hygrophorus (camanophyllus)

Pileus very much distorted from position of growth, surface fibrillose, watery almost tomentose, sepia, shaing to cinnamon-buII on margin; gills subdistant rather thick, white, becoming warm-bulf, osomewhat; interveined;stipe thick, much distorted, but gills decurrent, apparently collected along with Hygrophorus of same date(Nov.LL) $q \cdot 5137$., probably large, distorted specimen.

Specimens examined:

CostaRica, San Jose, Rancho Redondo, Alt. $2250 \mathrm{~m}$., Dodge \& Thomas 5348

abo 
Hygrophorus.

Pileus gregarious to cespitose, $7 \mathrm{~cm}$. diam., dry verrucosetomentose, cinnamon-brown, shading lighter toward margin;flesh thin, cartilaginous, continous with stipe; gills thiek, waxy, distant, narrow to medium, slightly decurrent, maiz-yellow; stipe hollow, fragile, cartilaginous, $7 \mathrm{~cm}$. \# $9 \mathrm{~mm}$., white, fibrous, sulcate.

Grassy potrero under Quercus.

Specimens examined:

Casta Rica, San Jose, Rancho Redondo, Alt.2250m., Dodge \& Thomas 
1. 


\section{Hygrophorus (Camarophylius)}

(See painting) Nothing like it in Murril.

Pileus campanulate, moist not viscid;flesh thin;gilis thick \& waxy, free or nearly so of moderate width; stipe hollow, splitting \& rolling inward, suggesting stem of Helvelloideae, white, molst, but not viscid; no trace of ring or volva.

Specimens examined:

Costa Rica, Cartago, Pejivalle, Alt.650-820,Dodge \& Thomas 4475 


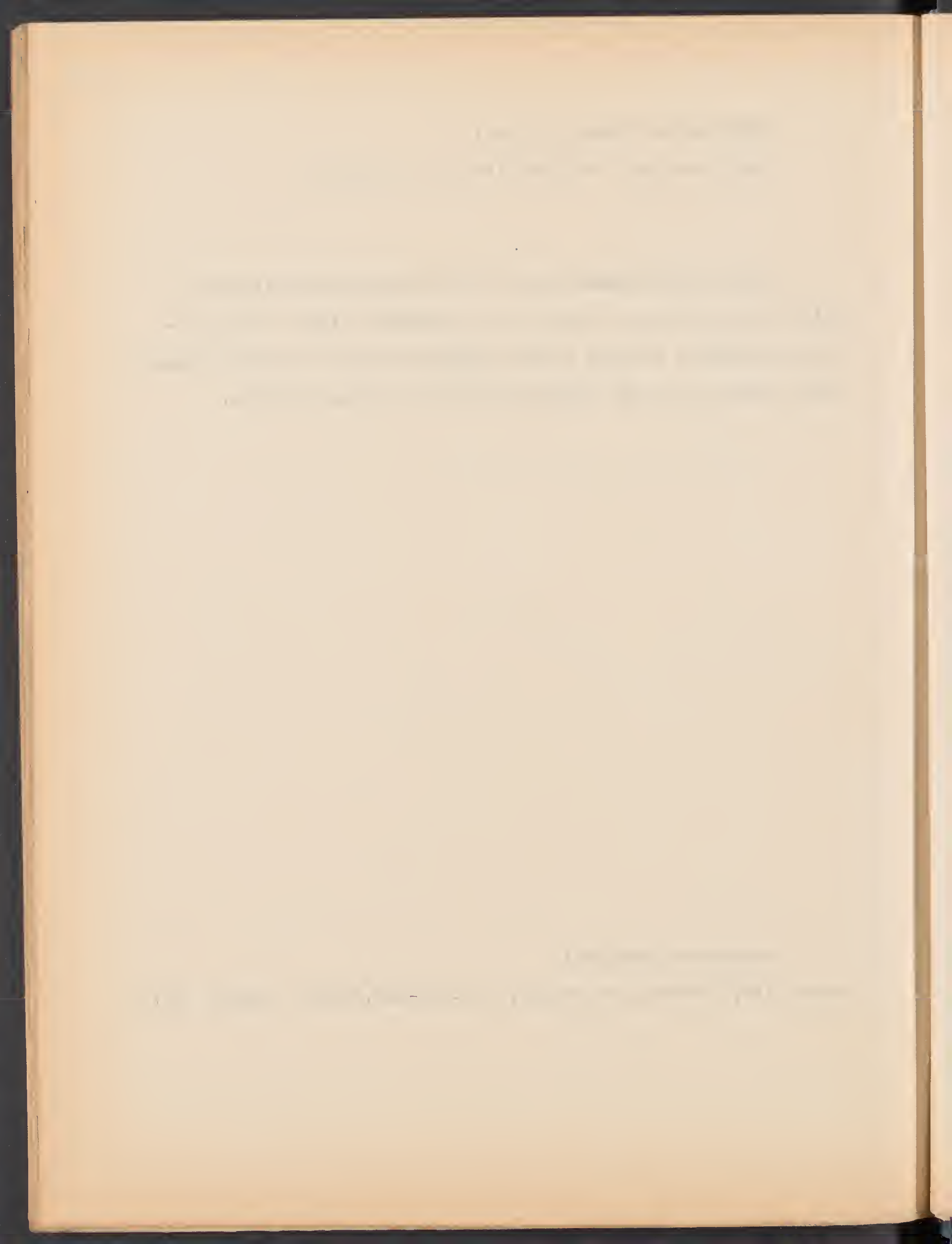


Hygrophorus nearest H. subpraetensig. M.

Pileus viscid, raw umber, expanded, margins slightly inrolled; gills waxy, distant, adnate, white; flegh $1 \mathrm{~mm}$. thick on disc; stipe silky above, slightly furfuraceous below, white.

\section{Specimens examined:}

Costa Rica, Cartago, Pejivalle, Alt.650-820m., Dodge \& Thomas 4474 
Hygrophorus

Pileus old, dry when collected, up to $1.5 \mathrm{H} 2 \mathrm{~cm}$. smooth \& shining (probably vitsctd), ochraceous-buff to ochraceous-tawny, hemispheric, margin inrolled; flesh very thick, white; gills adnate, decurrent by line, thick, subdistant, ochraceous-buff; stipe thick, fleshy, continuous with rlesh, fibrillose below, evanescent ring, becoming shining, (viscid) 15 \# $6 \mathrm{~mm}$.

Specimens collected:

Costa Rica, San Jose,Finca Santa Rosa, ALt.2580 m.,Dodge b2a7 Sumon: Waldeck ${ }^{40 \mathrm{mod}}$ Bodge 7196 
Hygrophorus (en Hyg.)

Whole plant surrounded by thick gel, viscid, universal veil; pileus unexpanded when young, $2.2 \mathrm{~cm} .$, sepia, shading to Isabella color; gills white, subdistant, waxy, adnate; stipe thick, floccose,pruinose above; gelatinous annulus, white, reticulate, striate with sepia, as gel dries, $4 \mathrm{~cm} \# 7 \mathrm{~mm}$. Mossy banks under Quercus.

\section{Speciraens examined:}

Costa Rica, San Jose, Rancho Redondo, Alt.2250m.,Dodge \& Thomas 5137 
Hygrophorus?

Pileus $3.2 \mathrm{~cm}$, convex, viscid, very dark chestnut-brown, margin appendiculate with universal veil, which fringes as triangular patches $2 \mathrm{~mm}$. long; gills thick, adnate, covered by partial veil which clings to edges of gills as pileus expands; stipe fleshy $5 \mathrm{~cm} \mathrm{\#} 7 \mathrm{~mm}$, tapering below to a fusiform bulb, fibrous, tomentose from universal veil in lower half, shining, silky, abowe white.

Potrero south of house.

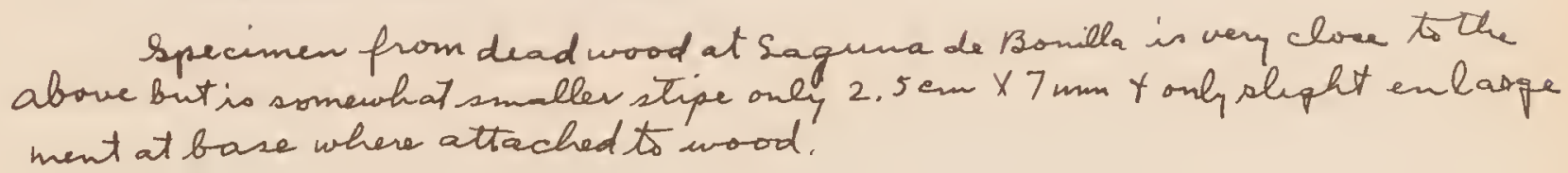

Specimens examined:

Costa Rica, San Jose, Rancho Redondo, Alt. 1160-1180 m.,Dodge

\& Thomas $5 / 36,5138$ 
Hysrophorus

(see paintings)

Among moss protonemata on bank of roadside.

Specimens examined:

Costa Rica, Aljuela, between Itiquis \& San Isiaro, Alt.980-1500m. Dodge \& Valerio 4752 
Hypholoma

Pileus degressed over stipe, apricot yellow, margin appendiculate ; flesh thick white over disc, very thin rest of way; gills adnexed, nearly free, cinnamon brown, broad, stipe fleshy hollow, irregularly compressed, light buff shading to russet, fibrous, subtomentose Mossy $\log$

Specimens examined:

Costa Rica: San Jose, Rancho Redondo, $2400 \mathrm{~m}$, Dodee \&Thomas 5407 


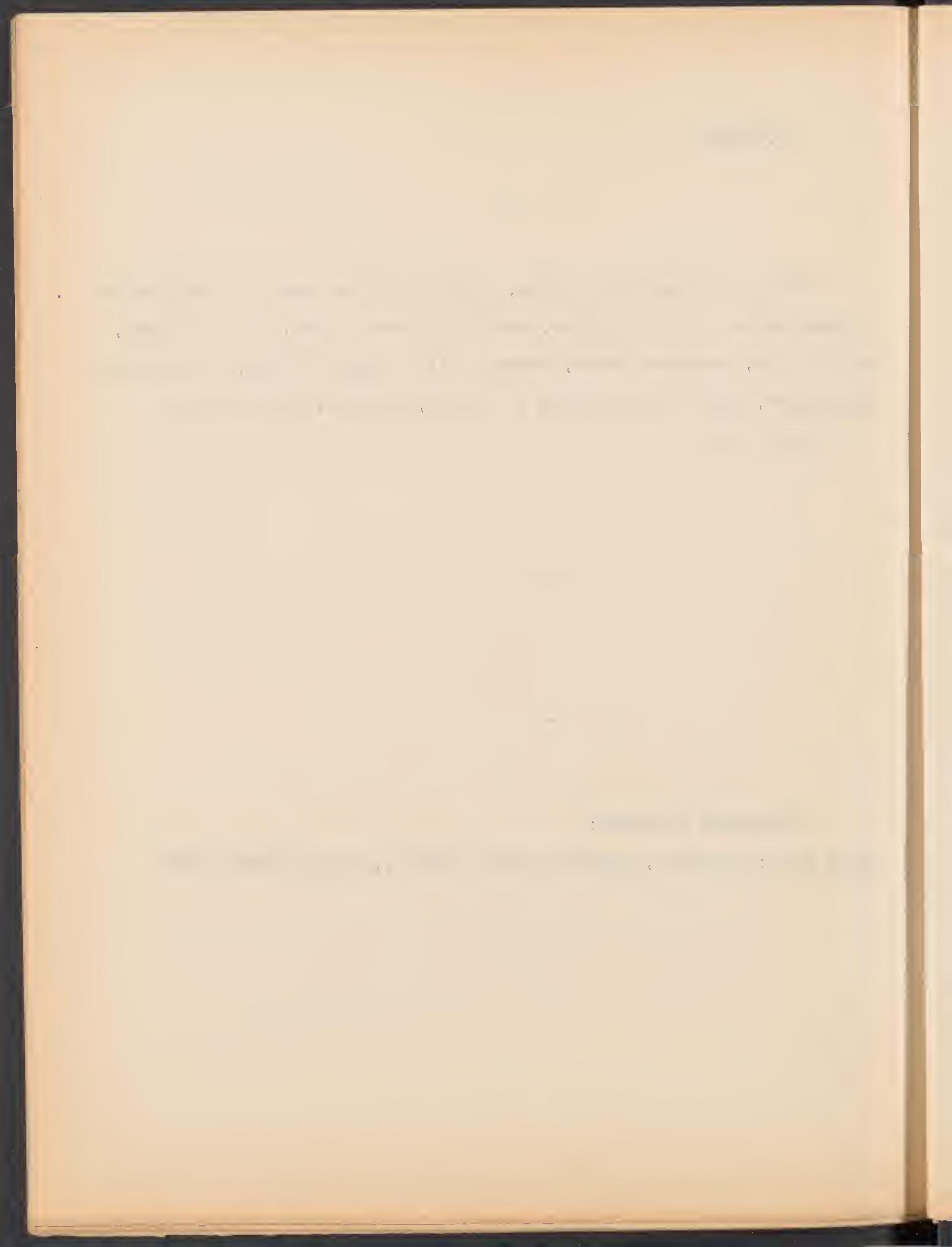


Hy pholorna

pilei ses,itose, hemispheric to cunvex, disc tawy shadint to buffy yellow, $3 \mathrm{~cm}$ diam. smooth marein aliendicul.ate; stipe

cills adnate, close, puriljsh krown, flesh thin; huff yellow fibrillose almost squmose, $30 \times 4 \mathrm{~mm}$, Iizht buff abowe, shadinu to tawny bejow.

Rotten Iog

Specimens examined

Costia Rica: san Joser, Santa Rosa, Dodge \& Thomas 5243 
$-192010.1$

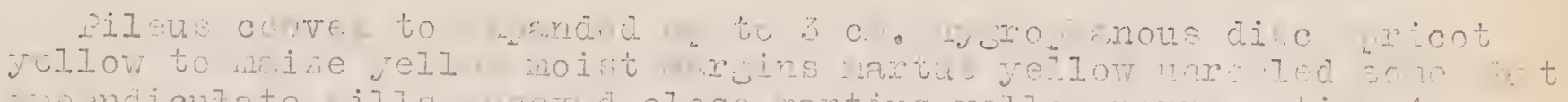

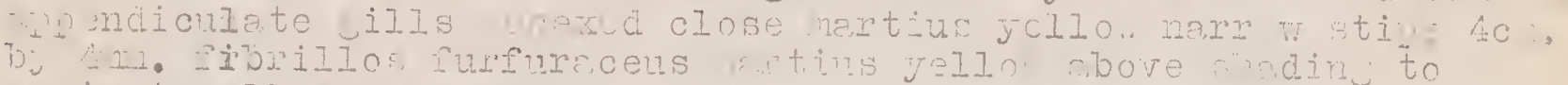

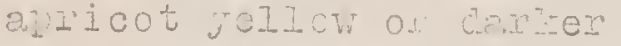

In soil votrero

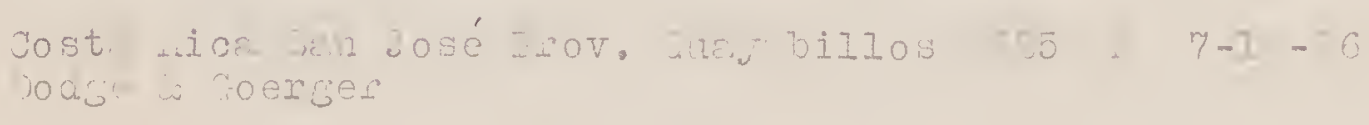


Hypholome usc oilas?

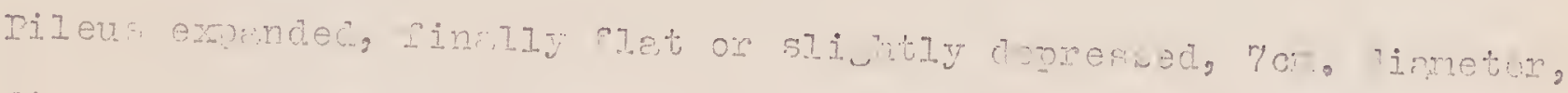
diso deep cinne con (rethon more yollow) corered by wool te, wi tore reinins of unitersel. veji (veil id iso conoolorous), color cramulty heconino Iichter to sinine hite, srooth mxein (shortuy strinte,

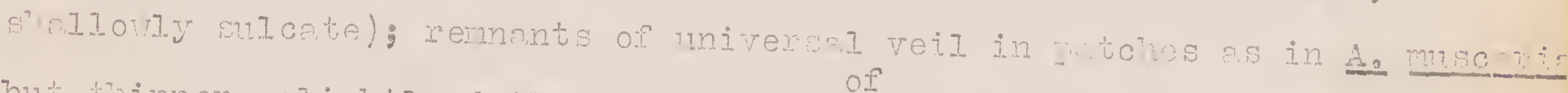

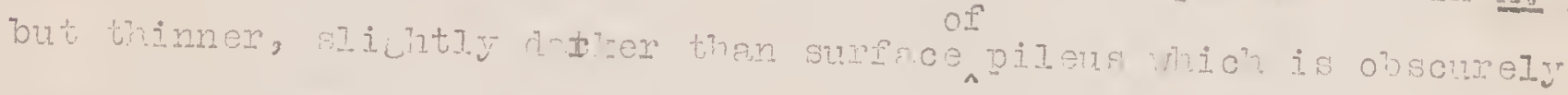
innote fibrous to near marein

vills rinuete, dnexed rentricose, color of Hroholome mhl teritum, crovded. IIesh $0.5 \mathrm{ch}$. thick at dish, thinnine crachaly to marcin stipe up to $13 \mathrm{~cm}, x 0.8-0.9 \mathrm{~cm}$, subeurle, silxy nite ebove, unle

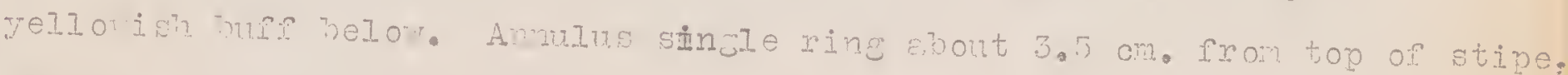
Hte, soon colored purplisin brot, erom sporer,

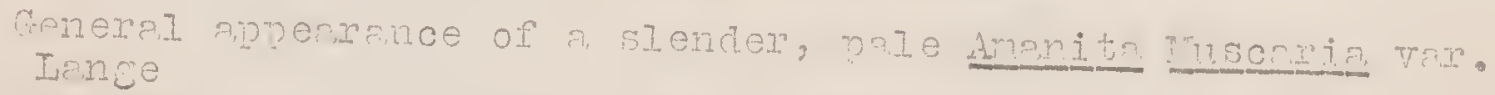
mericana, except for cills. Dase somemat hilbous, I. Ecm, golva adiote(if present), warcin or volve cottony?

Conal Zone, Jnlhoa, $6-1.5-30$

ordan 
Hy podendron

densely cesuldose ato base of stump, $2500 \mathrm{~m}$

Fileus hemisikeric to convex, mareins inrolued, fibrlilose

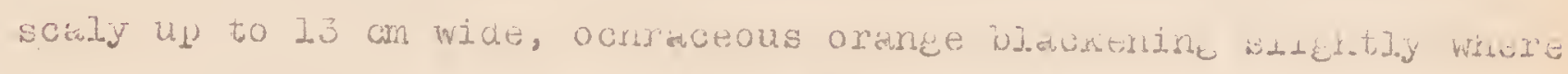

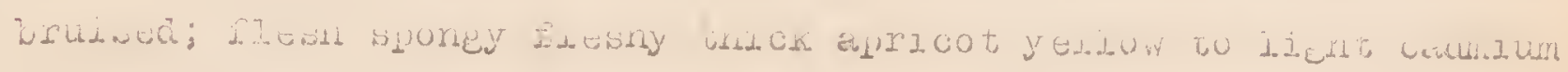

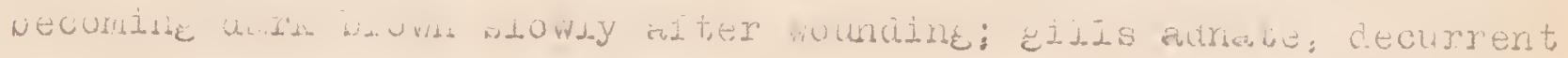

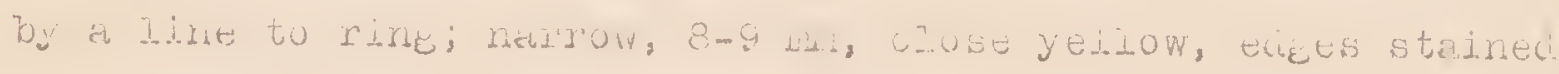

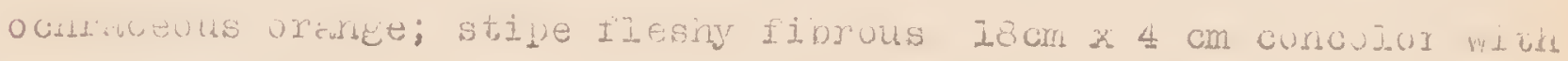
flesh, elirudus reduced to tilick suents; whost outer surface od

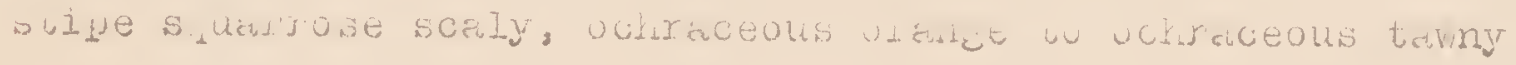
Specintenexardined

Coistes Rice: Ran Jose, Rancho Recurco m, Dodee \& Thornes $5 \% 53$ 





